\title{
Deployment of Mathematical Simulation Models for Space Management
}

Ausgeführt zum Zwecke der Erlangung des akademischen Grades eines Doktors der technischen Wissenschaften

\author{
unter der Leitung von
}

Ao. Univ. Prof. Dipl.-Ing. Dr. techn. Felix Breitenecker Institut für Analysis und Scientific Computing (E101)

$$
\begin{aligned}
& \text { eingereicht an der Technischen Universität Wien } \\
& \text { Fakultät für Mathematik und Geoinformation }
\end{aligned}
$$

von

\author{
Štefan Emrich \\ Matrikelnummer: 0026203 \\ Parkulica 32 \\ A-7304 Veliki Borištof
}


"In this new era of living within the means of one planet, GDP has become a less valuable indicator of progress."

- Global Footprint Network 


\begin{abstract}
Buildings are responsible for roughly one third of greenhouse gas (GHG) emissions within developed countries, yet space utilization efficiency is still a widely unidentified topic. With the sector only starting to become aware of it as a means to reduce GHG emissions and save (financial) resources there are hardly any strategies to improve space utilization efficiency.

Mathematical modeling and simulation (MMS) offers a wide variety of tools and approaches to analyze and improve systems and for producing answers to problems that cannot (or only at a very high "price") be answered in real life. Yet, at the time being MMS is generally not being used in the realm of real estate management.

This thesis' original contribution to knowledge is bridging the gap between space management and MMS, covering multiple aspects of the problem. First an introduction to the problem of space utilization and management, including identification of potentially interesting objects (educational facilities) and (legal) obstacles, is given. Next the classical definition of the MMS-process is expanded in order to reduce chances for misunderstandings and poor project-design in MMS-projects, followed by an evaluation of the mathematical options for improvement of space utilization, which shows a lack of practical solutions to solve real-world problems in this area (timetabling and scheduling). Subsequently the focus is shifted from timetabling to room allocation, for which a tool-set is assembled. This includes methods for system analysis, model-deployment and -integration and approaches for mathematical modeling of the respective systems. For system analysis the Business Process Modelling Notation (BPMN) is adapted for improved efficiency and the Stakeholder Analysis (SHA) process is formalized. For model-deployment methods from computer sciences (Entity Relationship Modelling) are transferred and combined with techniques from information visualization. Based on this a guideline for deployment of mathematical simulation models is created, which allows identification of obstacles for model-deployment within real-world systems and provides options for project modification to overcome those. Finally mathematical definitions are introduced, allowing formalization and quantification of the problems faced in space management.

This elaborated theoretic base is put to test in a case study at Vienna University of Technology (TU Vienna). In the course of the project it is found that the institutional obstacles (identified at the very beginning) hinder great improvement. Workarounds, based on the developed deployment guideline, still allow for a reasonable outcome of the simulation project. In the hope that financial savings are a sufficiently strong motivation for a thorough implementation in the private sector, an outlook is given for improvement of space utilization
\end{abstract}


in the field of office buildings. For this a dynamic, agent-based (AB) simulation model is developed and presented along with general results for this business area.

The thesis shows the big potential that MMS offers for improvement of space management utilization. Besides providing the tools for practitioners of both areas (MMS and real estate development) which should allow improved interdisciplinary cooperation, recommendations are given to overcome the (institutional) obstacles that remain. 


\section{Kurzfassung}

In den entwickelten Ländern Europas sind Gebäude für etwa ein Drittel aller Treibhausgasemissionen verantwortlich. Nichtsdestotrotz ist die effiziente Nutzung (hohe Auslastung) von Räumen ein weitgehend unbekanntes Thema, welches als Möglichkeit zur Senkung von Kosten und Emissionen von der Branche erst entdeckt wird. Bedingt durch diese Tatsache existieren bislang auch kaum Strategien zur Effizienzsteigerung in diesem Bereich.

Mathematische Modellbildung und Simulation (MMS) bietet eine breite Palette an Werkzeugen und Ansätzen um Systeme zu analysieren und zu verbessern, beziehungsweise um Antworten auf Fragen zu finden, die in der Realität nicht oder nur zu einem extrem hohen "Preis" beantwortet werden können. Bislang wird MMS jedoch im Allgemeinen in der Immobilienbranche nicht genutzt.

Der Beitrag dieser Dissertation liegt darin, die oben beschriebene Lücke zwischen Flächennutzung beziehungsweise Flächenmanagement und MMS zu schließen. Als Einleitung wird das Problem der Raumnutzung/des Flächenmanagements samt Identifikation von potentiell interessanten Objekten (Schul- und Universitätsgebäude) und der (rechtlichen) Hürden erörtert. Danach wird die klassische Definition des MMS-Prozesses erweitert, um das Risiko von Missverständnissen und schlechtem Projektdesign bei MMS-Projekten zu reduzieren. Es folgt eine Evaluierung der mathematischen Optionen zur Verbesserung der Flächennutzung, welche einen Mangel an praktikablen Lösungen für reale Anwendungsfälle auf diesem Gebiet (Timetabling und Scheduling) feststellt. Es wird daher der Fokus vom Timetabling auf Raum-Kontingentierung verlegt, wofür eine entsprechende "Werkzeugsammlung" entwickelt wird. Diese umfasst Methoden für die Systemanalyse, den Modelleinsatz und die -einbettung sowie Ansätze für die mathematische Modellierung respektiver Systeme. Für die Systemanalyse werden die Business Process Modelling Notation (BPMN) zugunsten besserer Lesbarkeit modifiziert und der Prozess der Stakeholder Analyse (SHA) formalisiert. Für die Unterstützung beim Modelleinsatz werden Methoden aus der Informatik (Entity Relationship Modelling) transferiert und mit Techniken aus der Informationsvisualisierung kombiniert. Darauf basierend wird ein Leitfaden für die Einsatzplanung entwickelt, mittels welchem Hindernisse bei der Modelleinbettung im Realsystem identifiziert werden und entsprechende Projektmodifikationen durchgeführt werden können. Schließlich werden mathematische Definitionen entwickelt welche es ermöglichen die Probleme der Flächennutzung zu formalisieren und zu quantifizieren.

Dieser entwickelte "Werkzeugkasten" wird in einer Fallstudie an der TU Wien angewandt und auf seine Tauglichkeit hin geprüft. Im Zuge dieses Projektes muss jedoch festgestellt werden, dass die (anfangs identifizierten) institutionellen Hindernisse den geplanten Einsatz behindern. Mithilfe des entwickelten 
Leitfadens können jedoch Behelfslösungen erarbeitet werden, welche zu einem vernünftigen Ergebnissen führen. In der Hoffnung, dass in der Privatwirtschaft finanzielle Einsparungen ein hinreichender Anreiz für eine konsequente Umsetzung von entsprechenden Projekten sind, wird ein dynamisches, agentenbasiertes Simulationsmodell für Büroflächen entwickelt. Dieses Modell wird zusammen mit allgemeinen Ergebnissen für diesen Anwendungsbereich präsentiert.

Die Dissertation zeigt das große Potential für die Verbesserung der Flächennutzungsintensität mittels MMS. Neben der Entwicklung und Bereitstellung von Methoden für Anwender beider Disziplinen (MMS und Flächenmanagement), welche die interdisziplinäre Kooperation verbessern sollten, werden Empfehlungen für die Beseitigung der verbliebenen (institutionellen) Hindernisse ausgesprochen. 


\section{Zahvalne riči}

\section{Hvala,}

vam dragi roditelji, dragi brati, draga obitelj i predse draga Evica. Bez vašega podupiranja i motiviranja bi prošla ljeta bila čuda teža. A tebi Evica i hvala da sam smio pisati dio ovoga djela kod tebe u Sibirskoj zimi Kemerovi.

Zahvalim se mojemu profesoru Felixu Breiteneckeru, ki mi nije omogućio "samo" disertaciju (i pred tim diplomsko djelo), nego se je i uvijek trudio istaknut širju sliku kraj granice univerziteta i struke i me je na tom putu poslao za projekt na ETH. Čim sam i jur kod mojega drugoga profesora Dietmara Wieganda, komu se zahvalim - osim za ugled u arhitekturu i menedžment projektov - za upoznanje s Frankfurtskom školom i njegov trening akribičnomu djelovanju. Oba dvim se zahvalim za veliku slobodu ku sam imao pri istraživanju i pisanju ovoga djela. Srdačna hvala Nikiu Popperu i kolegam iz dwh-a — bez njih i plodnih razgovorov uz, za i pod dwh-šonkom bi vjerojatno još uvijek nebi bio završio ovo djelo. Hvala i ostalim kolegicam, kolegam, prijateljicam i prijateljam skimi sam čuda puti diskutirao i tako našao svoj put kroz džunglu disertacije.

\section{Thank you,}

dear parents, brothers, family and Evica. Without your support and motivation the past few years would have been much more tiresome. Thank you Evica also for giving me the opportunity to write a part of this thesis when staying with you in Kemerovo's Siberian winter.

A big thank you goes to my professor Felix Breitenecker who not only gave me the chance of writing this thesis, but also for pointing out the importance of looking outside of one's area of specialization and over universities' horizons which eventually led me to work at a project at the ETH Zürich. Which brings me to my second professor Dietmar Wiegand, who I do not only thank for the insight into architecture and real-estate development, but also for confronting me with the Frankfurt School and for his training in meticulous work. I am grateful for the freedom that both professors gave me while researching and writing on this thesis.

A great thank you goes to Niki Popper and the colleagues from dwh - without you, and the fruitful discussions at, behind (and under) the dwh-bar I probably would still not have finished this thesis. Finally I thank all colleagues and friends for the countless discussions and talks which gradually lifted the fog and helped me find my way through the jungle of this dissertation. 


\section{Contents}

1 Introduction $\quad 1$

1.1 Eco-efficient buildings in a global context . . . . . . . . . . . 1

1.1.1 Global Warming and Green House Gas Emissions . . . . . 1

1.1.2 Buildings as Major GHG Emitters . . . . . . . . . . 3

1.1.3 Reducing Emissions via Space Management . . . . . . . . 6

1.1.4 Steps Towards "Efficient" Space Management . . . . . . . 7

1.2 The Complexity of Space Management . . . . . . . . . . . . . . 9

1.2.1 Building Operation - An Area of Conflict . . . . . . . . . 9

1.2.2 Benefits of Efficient Space Management . . . . . . . . . . 10

1.2.3 Pooling - Why Size Matters . . . . . . . . . . . . . . 12

1.2.4 Setting the Focus on Universities . . . . . . . . . . . . 14

2 From Model to Decision $\quad 21$

2.1 Modeling and Simulation . . . . . . . . . . . . . . 21

2.1.1 General overview . . . . . . . . . . . . . . . . 22

2.1.2 Defining Dynamic Modeling . . . . . . . . . . . . . 23

2.1.3 The Simulation Process . . . . . . . . . . . . . 26

2.2 Towards the problem's solution . . . . . . . . . . . . . . . . . 32

2.2.1 Mathematical Optimization ............ . . 32

2.2.2 Finding Good Enough Solutions . . . . . . . . . . . 36

2.2.3 Settling for Room Allocation Instead of Timetabling . . . 40

3 Methodology $\mathbf{4 5}$

3.1 System Analysis . . . . . . . . . . . . . . . . . . . . 46

3.1.1 Business Process Modeling Notation . . . . . . . . . . 51

3.1.2 Complementary Methods Used . . . . . . . . . . . 58

3.2 Integration of Model and Data . . . . . . . . . . . . . . . . . 64

3.2.1 Model Deployment \& Post-Processing . . . . . . . . . . . 64

3.2.2 Information Visualization . . . . . . . . . . . 70

3.3 Mathematical Modeling Concepts Applied . . . . . . . . . . . . 86

3.3.1 Discrete Event Simulation . . . . . . . . . . . . . . . 86

3.3.2 Cellular Automata (CA) . . . . . . . . . . . . . 90

3.3.3 Agent-Based Methods ............... 96

4 Application and Case Study 101

Genesis of the MoreSpace-Project . . . . . . . . . . . 101

4.1 System Analysis and Pre-Deployment . . . . . . . . . . . . 103

4.1.1 Definitions and Objectives ............. 103 
4.1.2 System Analysis of TU Vienna . . . . . . . . . . . . . . . 111

4.1 .3 Problems Encountered . . . . . . . . . . . . . . . . . . 124

4.2 Modification of Project . . . . . . . . . . . . . . . . . . 136

4.2.1 Adaption of Deployment . . . . . . . . . . . . . . 136

4.2 .2 Visualizing Room Utilization . . . . . . . . . . . . . . 142

4.3 Simulation of the Office Environment . . . . . . . . . . . 155

4.3.1 Space, Workplaces and Utilization . . . . . . . . . 155

4.3 .2 Model Implementation . . . . . . . . . . . . . . . . . 157

4.3.3 Simulation and Findings . . . . . . . . . . . . . . . 163

5 Conclusion \& Perspective $\quad 169$

$\begin{array}{ll}\text { Bibliography } & 175\end{array}$

$\begin{array}{ll}\text { Curriculum Vitae } & 183\end{array}$ 


\section{Author's note:}

Gender-neutral language is practically (almost) impossible to achieve — or ugly to read. Nevertheless, starting a text by saying something as "formulations apply to both sexes" and sticking to the male perspective does not improve things. Thus I chose to use either female and male forms throughout this thesis - with no intrinsic order. 


\section{Chapter 1}

\section{Introduction}

This chapter naturally serves as motivation for the present thesis - helping to deploy mathematical models within real estate development in order to aid in reducing the ecological footprint left by buildings. For this the connection of buildings and green house gas emissions is being laid out, together with an alternative perspective on the ecological footprint itself (section 1.1. With this perspective the complexity of space management is analyzed and universities selected as a worthwhile objective in section 1.2. In addition it also provides some background information helpful to understand subsequent development and reasoning (e.g. in section 2.2.3).

\subsection{Eco-efficient buildings in a global context}

\subsubsection{Global Warming and Green House Gas Emissions}

In their communication the (European Commission, 2009a, issued on January 28) states as follows:

"In order to limit the global average temperature increase to not more than $2^{\circ} \mathrm{C}$ above preindustrial levels, developed countries as a group should reduce their emissions to $30 \%$ below 1990 levels in 2020." (p. 2)

"To have a reasonable chance of staying below the $2^{\circ} \mathrm{C}$ threshold, global GHG [Green House Gas, author's note] emissions must be reduced to less than $50 \%$ of 1990 levels by 2050 . In addition, global GHG emissions, excluding emissions from land use, land-use change and forestry, will have to peak before 2020. Developed countries must lead in meeting this global goal and demonstrate that a low-carbon economy is possible and affordable. A significant contribution from developing countries, and in particular from economically more advanced developing countries, is also essential, as many of them are quickly becoming important emitters. To this end, cooperation must be significantly boosted to provide the necessary capacity, technology and finance." (p. 3)

Even though the impact and the influence of GHG emissions on climate are not completely undisputed, human influence on global warming (especially via 
GHG) has been called "very likely" by the Intergovernmental Panel on Climate Change (IPCC) Solomon et al. (2007). As the IPCC is a body connected to the $\mathrm{UN}$, that in 2007 (together with former US vice president Al Gore) was honored with the Nobel Peace Prize "for their efforts to build up and disseminate greater knowledge about man-made climate change, and to lay the foundations for the measures that are needed to counteract such change", their evaluation can be assumed well-founded.

In order to implement the drastic changes specified by the European Commission (EC) multiple strategies will have to be applied, ranging from high-tech research (e.g. carbon capture and storage, improvement of solar-power technologies and utilization of renewable energy sources), shifting of public funds (towards sustainable energy, products and traffic, to name a few) to simple energy savings (partially by the end-user) or even changes in habits such as the reduction of meat consumption due to necessary decrease of GHG-emissions by the meat-producing industry. It is clear that this process has to work top-down as it needs coordination and supervision, which means that policies and laws have to be created, adapted or even revised towards this ambitious goal.

The ecological footprint, though not completely without dispute, is a very useful characteristic to monitor the process towards this goal. The idea of the ecological footprint is to compare the consumed resources of individuals, nations or the entire world population with the world's (renewable) capacities. In other words it is "a resource accounting tool that makes sustainability measurable" Pollard et al. (2010). Thus a footprint where the division of a population's demand by earth's biocapacity leads to a quotient of 1.0 is the largest possible footprint for a sustainable society. Everything beyond a value of one describes an exploitation of world's resources and consequently must lead to a crash as humanity will sooner or later run out of resources.

The current ecological footprint for the world's (populate) continents can be taken from Table 1.1 (source of data Ewing et al. (2010a)). Due to a lag in data-collection, the numbers are from 2007. With a global deficit of -0.9 mankind is currently exploiting the world's renewable resources by far. And one can assume that the figures of consumption/capita have further risen (due to increased wealth) and those of biocapacity/capita declined (due to a growing global population) in the 3-year gap.

The currently negative situation becomes even worse if taking into account the future development. As the former "third world countries" emerge and increase their wealth, it is obvious that their consumption and hunger for resources will grow. The impact of such a tendency is expressed in Fig. 1.1, in which countries' human development index is plots against their ecological footprint. For reference two horizontal lines (available biocapacity/capita in 1961 and in 2007) as well as one vertical line (UNDP ${ }^{1}$ threshold for high human development) are added. In an ideal world all countries would be located in the square spanned by the axes, the 2007 biocapacity and the UNDP threshold. In the real world countries start to overshoot the earth's limits when reaching the UNDP threshold - at the latest.

As implied before, there is still an ongoing discussion of the definition, the calculation and the measurement of the ecological footprint and the carbon footprint (see Wiedmann and Minx, 2008), which accounts for roughly $50 \%$ of

\footnotetext{
${ }^{1}$ United Nations Development Programme
} 
Table 1.1: Overview of global and continents' ecological footprint with their consumption, biocapacity and reserve/deficit per capita (in global hectares/capita, source: Ewing et al. (2010a)).

\begin{tabular}{cccc} 
Region & Consumption & Biocapacity & Reserve/Deficit \\
\hline World & 2.7 & 1.8 & -0.9 \\
\hline Africa & 1.4 & 1.5 & +0.1 \\
Asia & 1.8 & 0.8 & -1.0 \\
Europe & 4.7 & 2.9 & -1.8 \\
Latin America $^{1}$ & 2.6 & 5.5 & +2.9 \\
US \& Canada $^{\text {Oceania }}$ & 7.9 & 4.9 & -3.0 \\
Oca & 5.4 & 11.2 & +5.8 \\
\hline
\end{tabular}

${ }^{1}$ Including the Caribbean

the total ecological footprint. Though in recent years significant efforts have been made in order to standardize the methods for calculation and to put them on a sound scientific basis (see Kitzes et al., 2008; Ewing et al., 2010b,a).

Without going into further detail of GHG emissions and related processes and issues, it is obvious that a significant reduction, of green house gas emissions (reported in $\mathrm{CO}_{2}$ equivalents) is necessary in order to reduce the ecological footprint of human society to a sustainable level. This is also expressed in the recommendations of the European Commission and the Intergovernmental Panel on Climate Control.

\subsubsection{Buildings as Major GHG Emitters}

One major sector contributing to GHG emissions are building. From available data and literature it is fairly hard, if not impossible, to find the exact amount of GHG emissions for which they are responsible. The reasons for this are diverse.

The changing definition of the sector and its contributors is one of them. Some sources exclude industry buildings and count those into the industrial sector. Other sources include emissions from the direct use of fuel as well as indirect emissions, which are emissions from heating- and power plants that offer community heating and electricity which is consumed. Some sources include the tertiary sector (offices, hotels, etc.) in their calculations. And yet another question is whether white goods (and other electricity consumers) are to be included or not.

Besides the varying definition of the sector, specific country differences make it hard to compare countries. For example did reunited Germany in 1990 have a vast amount of poorly insulated buildings and inefficient factories in its eastern part, the former German Democratic Republic. Austria on the other hand can cover a large share of its demand for electricity with hydroelectric power, which, being a renewable source of energy, substantially contributes to GHG emissions only during construction and deconstruction. And "southern" countries such as Portugal, Spain, Italy or Greece will need less energy for heating due to higher average temperature throughout the year. 


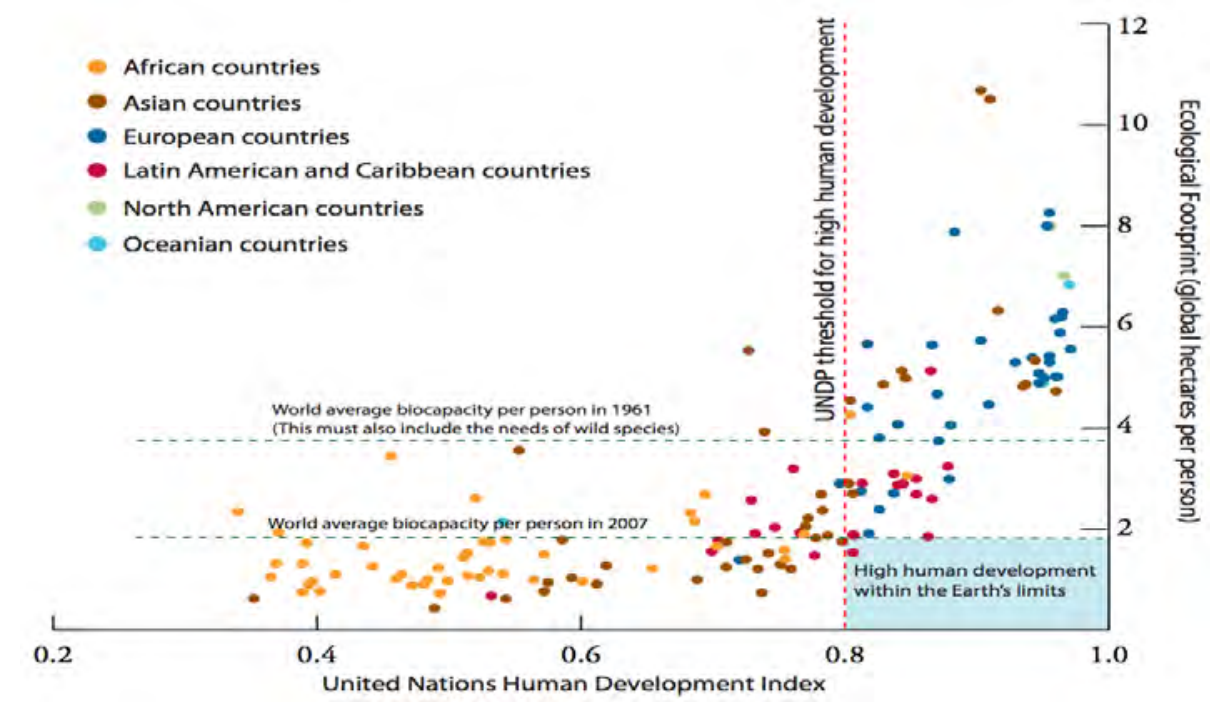

Figure 1.1: Scatter plot of world's countries' human development index (x-axis) versus ecological footprint (y-axis). Source: Ewing et al. (2010a, p. 21).

Further the lag of data collection (usually 2 to 4 years) constrains to using "old" data and finally the measurement units of collected data may vary as well (e.g. $\mathrm{CO}_{2}$ equivalent, Barrels of oil equivalent). Of course these units may be converted, but such conversions contribute to the overall inaccuracy. Thus while the figures available have to be treated with care, one can assume, that the order of magnitude is sound.

In order to get an impression of this magnitude, Table 1.2 lists the figures for the share of buildings GHG emissions within three countries (Belgium, Germany and Switzerland). These are derived from three different sources and published with varying units of measurement $\left(\mathrm{CO}_{2} \mathrm{e}\right.$, Boe and not specified). Another number is added by the European Commission via a press release from Commissioner Piebalgs, in which is stated that "buildings are responsible for $40 \%$ of energy consumption and $36 \%$ of EU CO2 emissions" (see European Commission, 2009b). And finally several websites related to the real estate industry also claim a share of $40 \%$ - although without citing their source(s).

Table 1.2: Share of GHG emissions produced by buildings, for three European states, with respective source of information and year published.

\begin{tabular}{ccccc} 
Country & Buildings' share & Unit & Year & Source \\
\hline Germany & $33.36 \%$ & $\mathrm{CO}_{2} \mathrm{e}^{1}$ & 2004 & Vahlenkamp (2007) \\
Belgium & $34.78 \%$ & $\mathrm{Boe}^{2}$ & 2005 & McKinsey (2009) \\
Switzerland & $40.00 \%$ & -3 & 2005 & $\operatorname{EnDK}^{4}(2005)$ \\
\hline${ }^{1} \mathrm{CO}_{2}$-equivalent in megatons (Mt) & & & \\
2 Barrels of oil equivalent & & & \\
3 Not specified in source & & & \\
4 & Konferenz Kantonaler Energiedirektoren und Energiefachstellen (2005)
\end{tabular}


Comparing these (five) numbers, the range varies from a third (Germany) to $40 \%$ (European Commission and real estate industry). Assuming a similar situation in other developed countries, substantial reductions of GHG emissions seem possible through improvement in the real estate sector.

In order to reduce GHG emissions in this sector several approaches appear worthwhile. A fast and currently very popular approach is the application of exterior insulation finishing systems (additional thermal insulation) to existing buildings. While this approach seems very straight forward, and is also often subsidized by governments, the used insulation materials are highly disputed. As published in Danner (2010) the market share of polystyrene-based insulation has increased considerably since 2007 in Germany - with the last available figure being $39 \%$ in 2004. Demolishing buildings insulated with such materials, which are manufactured from petroleum and contain flame retardants, becomes a very complicated task and produces toxic waste. Further criticisms include potential mold infestation through faulty insulation as well as the insulation preventing building walls to capture and store energy from the outside of buildings - mainly (direct) sunlight.

Another possible approach to quickly reduce GHG emissions of buildings is the installation of solar heating systems (for warm water and water-based heating) or photovoltaic units to generate "green" electricity. While the first approach is generally considered efficient and economic, the latter does strongly depend on the latitude and climate of the buildings location. On a macroscopic scale photovoltaic units - and other small scale power generators - pose a huge challenge for power networks that has not been solved until now. Thus, largescale deployment of decentralized photovoltaic cells on private housing does not seem to be promising yet.

A more time-consuming approach for the reduction of GHG emissions is to replace building components (such as windows) and technical equipment (e.g. boilers, air condition) with state of the art substitutes (in terms of energy consumption) at the end of their lifespan. Although economically reasonable, this approach is a fairly slow one. Besides, as stated in Dehli (2010), boilers and furnaces have reached an efficiency level at the edge of the physically possible, so that further improvements are marginal if an adequately sized condensing boiler is to be replaced.

Finally the most effective way to reduce emissions of buildings is to construct new buildings incorporating all available know-how and cutting edge technology (e.g. HVAC, smart building control, ...). Nevertheless the overall reduction of GHG emissions will be a very slow one, when taking into account a newconstruction rate for buildings of roughly $1 \%$ for the EU countries ${ }^{2}$, "which emphasizes the importance of the existing dwelling stock in achieving sustainability" (see Itard et al., 2008).

As a conclusion, the idea to quickly reduce emissions through passive houses or low energy buildings must be checked off. While on a long term scale a wise mix of previously described approaches (and yet to be invented technical solutions, as for example energy storage and decentralized power grids) will be necessary, alternative short term ways need to be found in order to reduce the emissions produced by the building sector.

\footnotetext{
${ }^{2}$ This figure should be taken with caution as the figures vary from country to country (between $0.5 \%$ and $2 \%$ in the year 2003) as do the types of estimates. For more detail see section 3.6 in Itard et al. (2008).
} 


\subsubsection{Reducing Emissions via Space Management}

A very elegant option emerges when buildings are viewed from a usage-driven perspective. In the western (European) culture it is common sense that buildings and rooms are serving certain tasks (e.g. a kitchen is used for cooking, a sleeping room for sleeping, a workroom for work, etc.). The idea to organize different "usage" temporally instead of spatially probably originated in ancient Japan, as described by Kasugai (1985, $3^{\text {rd }}$ thesis). While multi-purpose rooms are becoming more and more common in the western hemisphere, the concept is still far from being internalized. Nevertheless several pioneers haven been working on making this topic known to a broader audience. Among these are for example Joeckle et al. (1998), who saw the birth of the internet as the opportunity to administer multiple usage of built space by multiple users, or Wiegand (2007) who propagated this idea as one of the key means for sustainable buildings.

Following Wiegand's approach the (carbon) footprint of a building is connected to its benefit. The point of view upon a buildings' life cycle is thus not only cost-driven, but cost-benefit oriented. Subsequently this allows distribution of the buildings' emissions upon the benefit generated (e.g. people sheltered or "units produced").

In order to formalize this idea, the carbon footprint of a given building $b$ shall be denoted by $F P_{b}$ and divided by the produced units of benefit ${ }^{3}$ which are denoted by $U_{b}$. This ratio can easily be interpreted as the building's characteristic production costs $P C_{b}$ during the time interval $\Delta t$ (e.g. the buildings life cycle), and be written as

$$
P C_{b}(\Delta t)=\frac{F P_{b}(\Delta t)}{U_{b}(\Delta t)}
$$

The lower the production costs $P C_{b}$ are (in terms of GHG emissions or the carbon footprint respectively), the higher the building's carbon efficiency $C E_{b}$, which can be formally regarded as the inverse of the production costs

$$
C E_{b}=\frac{1}{P C_{b}} \text {. }
$$

To improve readability the time interval $\Delta t$ is omitted from the above and the following equations.

By replacing the production costs $P C_{b}$ in equation 1.2 with its expanded term from equation 1.1

$$
C E_{b}=\frac{1}{P C_{b}}=\frac{1}{\frac{F P_{b}}{U_{b}}} \quad \Longleftrightarrow C E_{b}=\frac{U_{b}}{F P_{b}}
$$

it becomes obvious, that there are two ways to increase the efficiency of a building: decreasing its emissions or increasing the units of benefit. With the latter usually being the easier parameter to tweak and thus the more realistic option, an improvement of a buildings carbon efficiency

$$
\uparrow C E_{b}=\frac{\uparrow U_{b}}{F P_{b}}
$$

\footnotetext{
${ }^{3}$ For now units of benefit shall remain an abstract term that will be discussed in more detail at a later point.
} 
can be achieved not only through modification of its carbon footprint (e.g. improved insulation or any other previously sketched approach) but through an increase in the "output" of its units of benefit $\uparrow U_{b}$.

As a building produces more units (of benefit) not only does its carbon efficiency increase, but it becomes (partially) obsolete to construct additional buildings which serve the same purpose - at least to that extent in which the existing building can satisfy demand. With this in mind the management of buildings, which is strongly connected to the productivity of the respective buildings, becomes a key factor in the quest for reduction of GHG emissions.

\subsubsection{Steps Towards "Efficient" Space Management}

The questions that arise out of this insight are diverse, with the most obvious probably being "What is productivity?" and "How can productivity be measured?" Which is also the reason why the word efficient is in quotation marks in the above heading. At first this might seem fairly straightforward to define and to answer when looking at production facilities. As soon as criteria that are hard to measure (e.g. working atmosphere or social factors) are introduced, the complexity of the system explodes and with it the difficulty of measuring the bottom-line impact.

Shifting the focus to buildings that do not serve production- or manufacturing-processes makes the above questions even harder to answer. It becomes necessary to define quantifiable measurement units in order to evaluate space management. Consequentially one wants to know whether the space management in place is already the "best" solution or if there is a way to improve it. Thus it obviously is not sufficient to just evaluate the management in place, but also to evaluate alternatives and compare those to each other.

It is unreasonable to compare the space management of two different buildings, eventually even serving different purposes. As it is unreasonable to change the space management in real life several times only to test its performance, another way for evaluation of various space management strategies has to be found. This is where (computer) simulation comes into play, as it allows thorough testing of virtually infinite possibilities at comparatively moderate costs and in very short time. A general introduction, overview and definition of dynamic computer-based modeling and simulation are given in section 2.1.

Assuming the right questions that should be answered by the model have been posed and, with respect to the necessary input-data, a model-layout has been defined accordingly. If such a model is to be used not only once for assessment of "the best" space management, but to be incorporated into a system for constant monitoring and (automated) planning, then the model's "place" within the systems processes needs to be found. This requires to identify the systems' stakeholders and the existing business processes - which has happened at least partially during systems analysis for the model generation - and, if necessary, modify theses processes in order to assure smooth integration (e.g. of data flow). Possible approaches and methods for the necessary systems analysis are covered in section 4 .

Provided a valid model that is properly embedded within its surrounding system produces verified simulation results. Unless these results are communicated in an understandable way, their benefit will be very limited - even though everything else, including information flow, has been done right. Thus it is 
necessary to extract the essential information (which may vary depending on the target audience) from the simulation results or simulation databases and visualize and interpret them in a beneficial manner. Such a processing should of course closely follow the initially posed questions and utilize suitable visualization techniques, as described in section 3.2.2. 


\subsection{The Complexity of Space Management}

\subsubsection{Building Operation - An Area of Conflict}

Without going into the details of the previously mentioned units of benefit yet, let's assume that these represent a kind of "individual space-use". Talking about real estate the following three stakeholders, i.e. involved groups with particular interests ${ }^{4}$, would represent a typical set: the owner or investor, the operator (of the venue) and the user/charterer. Of course other constellations are possible as well. Often two or three stakeholders might be united in one party, e.g. an operator also being the user (a company exclusively renting an office building) or companies constructing its new headquarters. In these cases the respective interests would (hopefully) coincide. As explained by Wotron (2009) one has to keep in mind that the stakeholders' interests are often diverging in such constellations.

Taking a conference venue as example, the user (e.g. a group of people having a meeting) will most likely be interested in not only having a comfortable sized location (neither too small, nor uncomfortably big) with all necessary infrastructure at hand, but also in paying a reasonable (low) price for this. The owner/investor of the venue on the other hand, will be interested in the maximum revenue. And the operator, located in between these two stakeholders, will of course be concerned with working out a margin as high as possible. This means either paying low fees/interest rates to the owner or generating a high income from the venue - or both.

Looking closer at it, it is obvious that this is a complex system in which the perfect solution strongly depends on the perspective, if it exists at all. There are different strategies which the stakeholders might pursue in order to achieve the best result, and these (strategies) can influence the decisions of the other stakeholders. An owner could for example try to build a venue as cheap as possible, neglecting infrastructure, insulation, etc. in order to keep the construction costs as low as possible. This would of course leave the operator with a building with high operating costs (and high GHG emissions) and poor infrastructure. As such a venue does not seem to be very desirable for the user, she will most likely expect a very competitive pricing. This again would reduce the operator's margin, forcing the operator to reduce operational spending (e.g. cleaning, heating, maintenance), leaving an even less desirable venue. Or the investor has to accept a decrease in revenue in order for the building to attract potential operators in the first place.

This example illustrates how the strategy of one stakeholder influences the whole system and thus the behavior of the other stakeholders. The applied strategies of course depend strongly on the information available to each of the stakeholders (e.g. the user knows all alternative venues with their respective pricing or operators are well-informed about the buildings quality prior to signing a contract), how the market looks like and also on the expectations/ assumptions of future developments. If there is only a single conference venue around (monopoly), the only question left to the charterer would be "to rent or not to rent?". But this leads way to deep into the theories of economics (complete vs. perfect information leading to the prisoners dilemma, leading to

\footnotetext{
${ }^{4}$ These do not necessarily have to be the same. On the contrary, in reality these will often be divergent and at least partially opposing.
} 
game theory and so forth), which shall not be covered in this thesis.

Extracting the essence, it has to be remembered that the stakeholders' interests are diverse and that the single "optimum" solution (if it exists at all) is a matter of perspective, most likely leading to a Pareto frontier or Pareto set. Such a set of solutions is characterized by the fact that further improvement for all stakeholders is not possible (this will be covered in more detail in section 2.2). Thus, in a struggle to reduce GHG emissions compromise solutions between the stakeholders will become necessary.

\subsubsection{Benefits of Efficient Space Management}

Following the reasoning so far, the primary goal of, or driving force for an efficiency increase through space management is the reduction of GHG emissions. But there are of course side effects, affecting the directly involved stakeholders as well as society as a whole. To answer the question whether these effects are positive or negative, one has to assume a certain perspective.

\section{Stakeholders' Benefits}

The stakeholders interests are, as sketched in the previous section (1.2.1), likely to be diverse if not oppositional. But still each one can profit of an efficiency increase in space management. Although it is necessary to apply a holistic view, as the space management (by definition) lies with the operator of a building or venue; the individual benefits grow out of the interaction between the stakeholders, based upon the efficiency increase (in space management).

To benefit the user the operator has to pack the positive effects into some kind of service. Staying with the prior example of a conference venue, the user might be more comfortable if she only has to articulate the demand and not (technical) specifications. E.g. a venue's "large ceremonial room" might look prestigious on paper, but be over-sized and kitschy for an IT-conference. If, on the other hand, the user specifies a demands such as "a meeting in a representative atmosphere for an IT-conference with 80 to 100 participants which will end with a casual dinner", the operator might be able to propose something more adequate.

For the operator a more efficient space management can translate into higher customer satisfaction (as illustrated above) but also make it possible to serve more customers (on the same are). Through this she might be possible to request a higher rent (relative to competitors) and in addition have more customers, further increasing her income. This again allows her to invest more into maintenance and cleaning, giving her further advantage over the competition, or to accept higher payment/interest rates towards the owner/investor, making it more likely to win a call for bids.

Subsequently an owner or investor benefits from an operator with an efficient space management through a potentially higher return on investment (ROI) and better maintained buildings. Knowing that such operators are harder to attract, the owner/investor might accept higher initial investments, resulting in a better building (with less GHG emissions) and thus a harder competition among operators in a call for tenders. Taking it even a step further, an investor could already search for an operator prior to construction in order to use the 
operator's know-how as input for the planning, resulting in even more efficient buildings.

Although such a scenario does, with respect to conservation of resources and reduction of GHG emissions, seem desirable, it probably requires establishing a (new or adapted) economic basis and development of the business models necessary in order for it to be successfully in a "free" market". Things look different if the focus is shifted from a free market to a controlled environment, i.e. an environment in which the stakeholders' individual interests are restrained in favor of altruistic goals (e.g. stakeholders are government bodies).

\section{Benefits for Society}

Besides previously stated reduction of GHG emissions, society as a whole can benefit of indirect savings induced by an efficient space management. If existing buildings can "produce" more, in terms of covering a larger share of the prevalent demand, construction of additional buildings serving the same purpose becomes obsolete - to the extent that is covered by the existent ones. This of course does not only save resources (funds, construction material, work force, etc.) but also avoids all GHG emission that originate (directly or indirectly) from the construction process and all emissions that these buildings would have produced.

Currently prevailing policies (based on continuous and unlimited growth) regard construction and production as generally positive, thus such savings and especially complete avoidance challenges these policies. This holds especially true for economically hard times, as with the current financial and economic crises $^{6}$, during which deficit spending usually becomes a frequently used term. Nevertheless, based upon the current situation and the predicted development of humanity, these policies will have to change in the (near) future in order for us to transform into a sustainable society ${ }^{7}$.

Picking up the controlled environments from before, a government can take advantage of such indirect savings and hand them on to the society. If it is not necessary to build, heat and maintain additional facilities (schools, hospitals, administration buildings, fire stations, etc.), the money saved could be used to improve services (e.g. to invest in better education, employ more medical personnel, buy newer equipment, etc.). ${ }^{8}$

Of course indirect savings (and derived benefits for society) are not limited to governmental bodies, but they are the most prominent and frequent ones to which these aspects apply, thus they shall be used as example. By utilization of pooling the savings effect can even be multiplied. As pooling will play a major role for the improvement of space management in a later part, its effect shall be illustrated by the following example.

\footnotetext{
${ }^{5}$ Knowing that completely free markets do not exist in practice, the term shall be used to refer to mixed-markets with a relatively high degree of freedom, such as those in the European Union.

${ }^{6}$ The financial crisis of 2007-2010 and the following European sovereign debt crisis, 20112013.

${ }^{7}$ With this thesis not being a philosophical or economic work it shall not dig deeper into these topics. Though the interested reader might find compelling ideas at Russel (2004).

${ }^{8}$ And finally, it will most likely be much cheaper to invest money into environmental protection now than to fight and deal with the effects of climate change.
} 


\subsubsection{Pooling - Why Size Matters}

Based on an administrative area - or catchment area, if you want - an imaginary pool is filled with all requests for a certain unit of benefit, this is the demandpool. It is obvious that the size of the pool is directly proportional ${ }^{9}$ to the size or population of the underlying area. If this pool of demands is now to be processed by servers (e.g. hospitals, administrative offices or schools) the ratio of pool size and number of servers strongly influences the amount of play.

To clarify this a few assumptions are made. The observed (total) administrative area is a quadratic field, with regular grid, dividing it into 16 squares, each representing a single household (the 16 squares in Fig. 1.2), which are to be supplied with schools. The number of children of school age which are within a household is a uniformly distributed, random variable $C$ from the discrete set $[0,1,2,3,4]$. Written as a table, the distribution would look as follows:

$$
\begin{array}{rccccc}
\text { Children per household } & 0 & 1 & 2 & 3 & 4 \\
\text { Probability } & 0.2 & 0.2 & 0.2 & 0.2 & 0.2
\end{array}
$$

The number of children within the households (each circle in Fig. 1.2 represents a kid within the respective household), was generated using the MATLABcommand

floor $(\operatorname{rand}(16,1) * 5)$

which returns a vector of length 16, satisfying the definition of our random variable $C$. The configuration was chosen at random. The circumstance that the overall mean of children per household being 2.0 is incidental but nice.

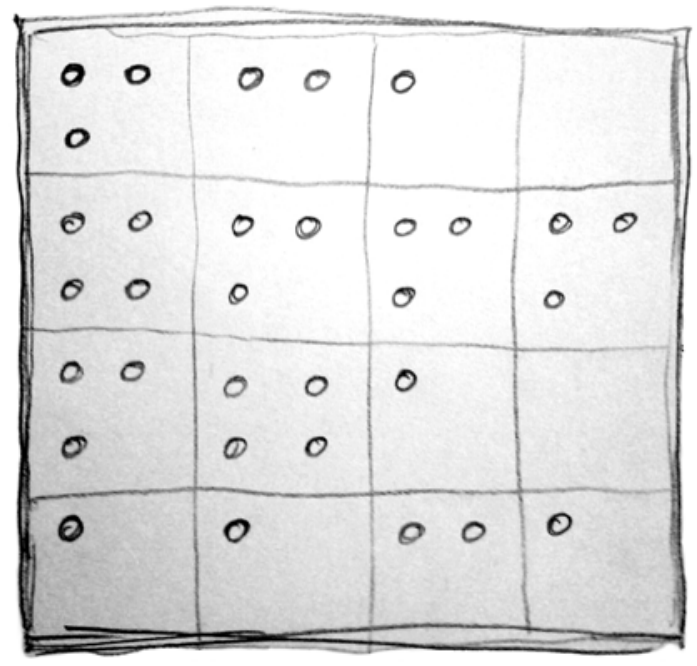

Figure 1.2: Total administrative area with 16 households and number of school children per household.

The smallest size for the school-pool is 1 household/school, in which case 16 schools would have to be built. In order for all children to be educated, each

\footnotetext{
${ }^{9}$ Arguably not in the mathematical meaning of "directly proportional", but in its colloquial general tendency.
} 
school would have to have the size adequate to the maximum number of children that a household could possibly have, with $\max (C)=4$. Thus the total area would have 16 schools with a total of 64 places for students, with only $12.5 \%$ of the households needing such a large school. Which is due to the stochastic nature of random numbers. In a perfectly uniform distribution a fraction of $20 \%$ of the households would need such a large school.

In an attempt to increase efficiency, always two neighboring households are being pooled and served with only one adequate school. These schools would still have to offer space for two times the maximum number of children (being 8). Continuing this enlargement of pools, the schools size would have to grow to 16 students for four households, 32 students for eight households and 64 students if all 16 households are thrown into a single pool (see Fig. 1.3). This is the point where statistics enter the stage.

At a certain pooling size, one can take advantage of the expected value $\mathbb{E}$ which for our case (of a finite and discrete random variable) is defined as

$$
\mathbb{E}[X]=\sum_{i \in I} x_{i} * p_{i},
$$

with $\left(x_{i}\right)_{i \in I}$ being the $i^{\text {th }}$ value $X$ can take on and $\left(p_{i}\right)_{i \in I}$ the probability of this happening (and $I$ a countable index-set). For our random variable $C$ with the above probabilities for a household having 0 to 4 children, the expected value hence calculates as

$$
\begin{aligned}
\mathbb{E}[C] & =\sum_{i=0}^{4} c_{i} * p_{i} \\
& =c_{0} * p_{0}+c_{1} * p_{1}+\ldots+c_{4} * p_{4} \\
& =0 * 0.2+1 * 0.2+\cdots+4 * 0.2=2.0 .
\end{aligned}
$$

Thus this allows for the school size to shrink from 4 students per household down to 2 /household - given a sufficiently large population.

But what is the effect in the present example of 16 households, is there already a smoothening effect? For this it is necessary to evaluate the difference among the pools. Knowing that the expected value of children in every household is two, the relevant figure is the deviation from this expected value. To be precise, one wants to know, how far the average household size within a given pool is off this value of 2 .

This question is answered by the standard deviation $\sigma=\sqrt{\mathbb{E}\left[(X-\mu)^{2}\right]}$, where $\mu$ denotes the mean of the random variable $X$ (being 2 for $C$ ). The standard deviation is applied to the averages of children per household in every pool and subsequently compared among pool sizes. The pools are defined according to Fig. 1.3. The numbers of children inside a pool, as well as the average per household for every pool as well as the respective standard deviation are presented in Table 1.3.

In the given case (discrete random variable) the standard deviation is computed using the overall mean/expected value of 2 children (instead of taking the pool-size's own mean). Thus the exact formula used to obtain the results of Table 1.3 writes as

$$
\sigma=\sqrt{\frac{1}{n-1} \sum_{i \in I}\left(x_{i}-\mu\right)^{2}}
$$



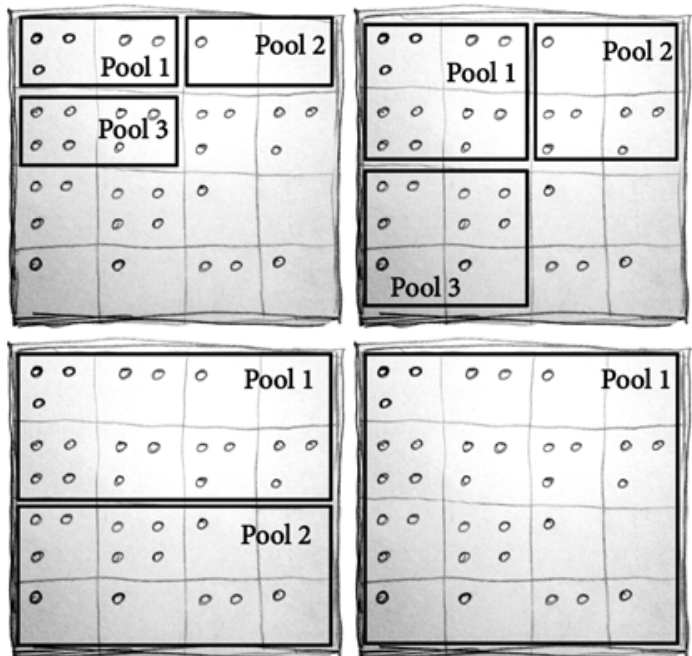

Figure 1.3: From left to right, top to bottom: Pooling with 2, 4, 8 and 16 households per pool.

with $n$ the number of pools in the set of pools $I, x_{i}$ the average children per household in the $i^{\text {th }}$ pool and for all sets $\mu=2$, the expected value of $C$ 's underlying distribution.

It is obvious, that the growing pool size significantly reduces the influence of statistical dispersion and subsequently allows organizing things with a much higher efficiency. Nevertheless, there is no guarantee whatsoever that the average number of children per household will converge towards the expected value and thus it will usually be desirable to include a certain margin when sizing the facilities. The margin's size of course depends on the resources available and/ or the willingness to provide them. In the present example of a governmental service, this usually translates into the availability of a budget and the priority which is given to the respective service by the administration.

Being an example aiming solely at illustration of the pooling effect, it intentionally neglects all other aspects. These are for example the availability of the provided service, especially in terms of distance to the service. Economies of scale represent a second aspect, actually favoring bigger pools. On the other hand this can lead to ever bigger facilities up to the point where they become un-administrable or psychologically negative (e.g. no personal touch - small is beautiful).

\subsubsection{Setting the Focus on Universities}

Space management in terms of organizational management (in contrast to individual optimization of space use) is of course not sensible everywhere. It is unlikely that space use within a residential building (i.e. its inhabitants) can be organized in a more efficient way trough external management. Further, as stated in section 1.2.1, the economic foundations for cooperative business strategies between the involved stakeholders don't exist at present. Without these foundations, it is possible, but fairly abstract to investigate ways to in- 
Table 1.3: Pool-size in households (HH) and numbers of children per pool, pool-average $\bar{x}$ of children/HH and standard deviation $\sigma$ of pools' means (according to Figs. 1.3 and $1.2)$.

\begin{tabular}{cccc}
$\mathrm{HH} /$ pool & Children in pool & $\bar{x}$ (pool average) & $\sigma$ \\
\hline 1 & $3,2,1,0,4,3,3,3$ & - & 1.3166 \\
& $3,4,1,0,1,1,2,1$ & & \\
2 & $5,1,7,6,7,1,2,3$ & $3.5,0.5,1.0,1.5$ & 1.2817 \\
4 & $12,7,9,4$ & $3,1.75,2.25,1$ & 0.8416 \\
8 & 19,13 & $2.375,1.625$ & 0.5303 \\
16 & 32 & 2 & - \\
\hline
\end{tabular}

crease the efficiency of space management. This leaves governmental facilities as potential targets, especially such, in which all stakeholders involved can be attributed to the government.

\section{Educational Facilities' General Potential}

Educational facilities are such a section of buildings where all involved stakeholders are attributed to the government — owned by the government (or stateowned subsidiary enterprises), operated by (governmental) school/university authorities, used by the public (students) and government employees (teachers and professors). Adding the government as the policy maker for education and the public as beneficiary of any improvement, this constellation seems an ideal one. One can assume that finding a consensus between stakeholders should be easier than in other constellations and successful test-implementations could be broadly adopted and implemented. Further, according to BIG (2013) educational facilities are the largest segment of publicly owned buildings, making up $64 \%$ (4.5 million square meters) of the portfolio of the "Bundesimmobiliengesellschaft" ${ }^{10}$. Thus improvements in this section accumulate to a big overall effect.

And yet another detail adds to the charm of these buildings: their mostly low utilization, as analyzed by Wiegand (2012) and in studies such as Prirsch (2012) or HEFCE (1999). This low rate of utilization is regularly contrasted by news reports of overcrowded schools and huge class sizes, or even by inquiries among teachings staff, according to which the lack of - financial or spatial - resources is often the reason why teachings cannot be scheduled (see Woplatek, 2010). Woplatek states that" "the presence of sufficient resources, regardless whether

\footnotetext{
${ }^{10}$ The Bundesimmobiliengesellschaft, short BIG is a quasi-governmental company managing Austrian publicly owned real estate

${ }^{11}$ Translation by the author, original wording: "Das Vorhandensein ausreichender Ressourcen, egal ob es sich dabei um finanzielle Mittel oder beispielsweise auch die Verfügbarkeit von Räumlichkeiten handelt, stellt für die befragten Personen aber eine wichtige Voraussetzung für das Zustandekommen des Muttersprachlichen Unterrichts dar und oft scheitert die Durchführung an dieser Problematik."
} 
monetary or the accessibility of space $[\ldots]$ is an important precondition for teaching [ ... ] being made possible, and often a deal-breaker." This leads directly to the core of space management.

Taking into account usual teaching times, vacations and holidays, the general utilization of educational facilities is dramatically lower than intuition might suggest. This of course does not imply that it would be best to lock students into educational facilities for as long as possible, cheating them out of their free time in order to increase utilization. On the contrary, regarding students and faculty as the user or charterer of the facilities, one of the goals of and characteristics for good space management has to be enabling highest possible quality of teaching - in terms of providing adequate time slots and infrastructure.

Intuitively, if there is no change in business hours, one would assume that there is limited possibility to increase the utilization of a building regarded as used to capacity during normal operation. For now the notion of "being used to capacity" shall be ignored (and covered at a later point) with the focus left on the change of business hours. As pointed out educational facilities are (virtually ${ }^{12}$ ) state property. Thus the government might as well use them for other than educational purposes if the types of use don't conflict. For example would it be possible to hold municipal council meetings in schools instead of building a separate assembly room. This might not be feasible for larger cities with regular meetings requiring special infrastructure and with meetings during normal working (and thus teaching) times. But it most likely would work out for smaller municipalities with meetings taking place on weekends or in the afternoon. The same applies to church choir practices or meetings of voluntary fire departments and the like.

As these examples suggest multi-purpose utilization can help increasing building efficiency. But there are obstacles to this of different nature, with the three most relevant categories probably being the "psychological", organizational and legal aspects. The first category includes things such the attitude of involved people towards abdication of special rooms, which is often seen as a status symbol or interpersonal friction in general. Secondly questions addressing the practical aspects of space management (e.g. body in charge of scheduling and the preparation/removing of infrastructure, or priority in case of collisions) need to be solved. And finally all aspects have to be covered legally, with schools being a very delicate environment with the potential worst-case scenario of child abuse.

\section{Universities' Advantages}

Many of the above mentioned obstacles apply to educational facilities in general, but are much more pronounced in schools than in universities or colleges, making the latter more favorable subjects. Beyond this universities also show a higher degree of freedom when it comes to operating times and they are (usually) much larger than schools, thus making pooling an interesting instrument for management. The larger scale usually carries on in terms of equipment, infrastructure and subsequently functionality of space, with university space in general - being much more versatile than space in schools.

\footnotetext{
${ }^{12}$ In Austria they are in general property of the previously mentioned, state owned enterprise Bundesimmobiliengesellschaft (BIG), but one could also think of other situation such as for example "sale and lease-back".
} 
Analyzing the aforementioned obstacles the psychological aspect is much reduced by the fact that universities are - as a rule of thumb - located in large urban settlements and themselves complex systems, usually having departments specialized on administrative tasks. Thus multi-purpose use of the facilities differs strongly from the sketched school arguably located in a rural community or small town. One can assume that external users approaching a university will be aware of entering a self-governing institution and behave accordingly.

Through the universities administrative departments the organizational obstacle can be also regarded as tackled - in principle. In practice, especially in countries in which universities are is still operated in a very bureaucratic fashion, the organizational aspect will most likely require real reorganization, change of processes and attitude. Or as Scott (1998) put it:

"It is far easier for would-be reformers to change the formal structure of an institution than to change its practices. Redesigning the lines and boxes in an organizational chart is simpler than changing bow that organization in fact operates. Changing the rules and regulations is simpler than eliciting behavior that conforms to them."

Being a governmental institution, this aspect is naturally closely connected to the legal bearings and the mission with witch universities are assigned by those. A very fundamental example for this is the question, if such institutions are even allowed to let other parties use their facilities at all.

Even though, according to Austrian law ${ }^{13}$, schools are allowed to let their facilities to external parties, the administrative effort for this makes it partially unpractical. As stated above, universities can be assumed to possess administrative capacities, even though these might not (yet) be geared to this purpose.

\section{Current University Organization in a Brief Historical Context}

This thesis is certainly not the place to plunge into the historical development of universities up from medieval times. Yet, in order to grasp the complexity behind ostensibly simple organizational changes, it is necessary to have a minimal understanding of the historical context of the subject. As major influence upon to today's situation can be attributed to the last two significant amendments of university laws - also referred to as reforms - which took place in 1975 and 2002 , these events will be covered briefly ${ }^{14}$.

Based upon Burtscher et al. (2006) the university systems - for the times before and after these reforms - can be described as follows:

1. Prior to the UOG $1975^{15}$ the university system had structures as found

\footnotetext{
${ }^{13}$ Precisely through $\$ 128$ SchOG, which stands for Schulorganisationsgesetzt and literally translates to "school-organizational law". This law does not apply to universities, but prior to 2002 the legal situation of universities was similar to that of schools today, as they were (respectively are) legally dependent institutions with partial legal competence. The current situation of universities together with its emergence and implications will be covered in the next two sections.

${ }^{14}$ The here presented information on the historical context as well as on the implications of the reform in 2002 is an attempt to excerpt the essence of Burtscher et al. (2006), necessary for basic understanding. Nevertheless, I strongly recommend reading the whole text to everybody that strives for a deeper understanding of the Austrian university system and the inherent difficulties.

${ }^{15}$ UOG abbreviates Universitätsorganisationsgesetz, which literally translates to "university organization law".
} 
in a corporative state ${ }^{16}$, with the professors holding extensive rights and individual freedom inside of the university system and external regulation coming from the federal government.

2. The system's reform through the UOG 1975 was coined by the protests of 1968 and needs to be seen in the light of those events, with a strong wish for transparency and participation. The amendment gave voting power to the non-professorial scientific personnel, non-scientific employees and even students, but basically did not change the rigid structures, while granting further influence to the federal government.

3. With the 1990ies and the emergence of new public management came what can be seen as antipole to the 1968 criticism of authority and the establishment - pressure to re-organize universities according to marketoriented ideas, inspired by the Anglo-American model. This culminated in the subsequent reform carried out by amendment of the UG $2002^{17}$.

As Burtscher et al. illustrate, the changes of the reform of 1975 are of especial interest, because although they shifted the balance of power towards the socalled Mittelbau, the non-professorial faculty, there was - as mentioned above no change of the Estates of the realm. The Mittelbau was still left without an exit-option (as becoming professor was only possible to very few of them), but now they had a voice. Further, universities were rationalized paving the path for modern mass universities.

The impacts of the 2002 reform are in this respect problematic, as they consistent with new public management - introduce an extensive centralization of the system, bundling powers within the rectorate (management). This of course means that the UG 2002 is stripping the Mittelbau of their voice and influence. On the other hand, the UG 2002 does not offer any exit either, which is fairly likely to lead to discontent among the affected groups.

Further Burtscher et al. argue, that the government secured its influence on universities through the regulations on how the Universitätsrat ${ }^{18}$ is being put together. This procedure ${ }^{19}$ requires the senate to set the size of the Universitätsrat to 5, 7 or 9 members, which have to fulfill some general criteria. Of these members $50 \%$ minus one person are filled (via elections) by the senate, and the same number of people are appointed by the government (on proposal of the responsible secretary). But, the "government-members" have to be designated after the election of the "senate-members". The final person of the Universitätsrat has to be decided upon amicably by the " $100 \%$ minus one". It has to be added, that only persons are eligible as member - besides the aforementioned general constraints -, which do not have and have not had an employment relationship with the university or the government for the past four years ${ }^{20}$.

\footnotetext{
${ }^{16}$ The term ständestaatliche Struktur would describe the model best, but is very hard to translate. Thus the probably best fit is "the structures of a corporative state" or the so-called Estates of the realm. Another suggested translation - actually a description - would be that of universities controlled by tenured faculty.

${ }^{17} \mathrm{UG}$ abbreviating Universitätsgesetz, literally "university law".

18 Universitätsrat roughly translates to "university advisory board". It is one of the highest institutions of every university next to the senate, the rector and rectorate. Its task can be compared to that of a supervisory board of a (commercial) company.

${ }^{19}$ According to $\S 21$ Abs. 6 Z. 3 \& 6 UG 2002.

$20 \S 21$ Abs. 16 Z. 4 UG 2002
} 
To describe the motivating ideas and intention for the UG 2002, Burtscher et al. use the term fracasomania ${ }^{21}$. This characterizes a high sensibility for a given (referred to as "local" by them) system's weaknesses but a blindness towards its strengths, which leads to comparing the bad or worst sides of the local system with the best ones of the alternative. On the other hand fracasomania has an inherent tendency to misunderstand the alternative model and subconsciously keep inadequate elements of the local system while overlooking the importance of necessary requirements (especially historical ones).

A very good example for fracasomania within the UG 2002 is the $\S 4$, through which universities became legal entities under public law with full legal competence ${ }^{22}$. This is aiming at giving high autonomy (with management-like structures) to universities in order to participate in the international competition and match with elite institutions. With the misjudgment being that this can or should be achieved solely through independence and market-like management structures, neglecting the fact that international elite-institutions are well in funds, contrary to Austrian universities which are chronically underfunded. Even worse, the expectation is not only that they will be able to compete on top levels, but also that they will need less governmental funding, since they are now equipped with management structures.

This brings up the topic of university property, which could be used to generate funds, as it is for example by the British top institutions. Those do not only posses buildings, but sometimes considerable amounts of land often in prime areas. Austrian universities on the other hand do neither own land nor the buildings they are using. In this respect the UG 2002 basically only stipulates ${ }^{23}$ that the universities are the legal successor with regard to the tenancy agreements and the right of use.

To complicate things further, the university property is not owned by the government directly, but - as previously mentioned - by the Bundesimmobiliengesellschaft $(B I G)$, a state-owned real estate company. One of the main factors to outsource the state-owned real estate and all tasks surrounding it mainly maintenance and administration - was to (optically) improve the federal budget. Even though this state-owned company was established with the goal to cover the federal government's demand for space ${ }^{24}$, the law explicitly states that this has to be done "according to market conditions and if economically justifiable" 25 .

This leads to the fact that the government owns a real estate company that is assigned to work profitable. At the same time almost $100 \%$ of this company's tenants are of governmental nature, which means, that the government has to equip these tenants with sufficient funding to pay the rents which, by law, need

\footnotetext{
${ }^{21}$ The term was coined by American sociologist and economist Albert Otto Hirschman in a Latin-American context as: "the conviction that all attempts at solving the nation's problems have ended in failure ... By invoking the desperate predicament in which people is caught, as well as the failure of prior attempts to reform, it is implicitly or explicitly argued that the old order must be smashed and a new one rebuilt from scratch regardless of any counterproductive consequences that might ensure" (see Hirschman, 1991)

${ }^{22}$ Translation by the author. Original, German term: vollrechtsfähige juristische Personen des öffentlichen Rechts.

${ }^{23}$ UG 2002 "VI. Teil" $§ 117, \S 118$ and "VIII. Teil, 5. Abschnitt" $§ 137-\S 140$.

${ }^{24}$ Defined in $\S 4$ of the Bundesimmobiliengesetz, roughly translated as "law of federal real estate".

${ }^{25}{ }^{2} 4$ Abs. 2 "[ ...] zu marktkonformen Bedingungen, und wenn es für sie wirtschaftlich vertretbar ist [...]", translation by the author.
} 
to be customary in the market. With some of the federal tenants, namely the universities, being legal entities that need to economize, one can easily imagine that this constellation is building up unnecessary friction and bureaucracy. This is of course counterproductive in a system where all stakeholders (should) pursue a common goal: generating benefit for the entire society, ideally through synergistic effects.

As universities have to fulfill an essential role for a nation - educating people and thus securing its future productivity and creativity - Ridder (2006) argues that universities must not be looked upon and treated as companies but as vital governmental institutions and resource.

\section{Conclusion}

Summing up, educational facilities, especially universities, appear to be a suitable target for increasing space management efficiency, thus the focus of this thesis shall be set on universities. With a single "owner" it should be possible to install a tested and proven method in all buildings/universities, which would not only allow a more efficient use of space in general, but also discharge the chronically strained budget of the department for education. Further it would help reducing $\mathrm{CO}_{2}$ and GHG emissions - a goal that otherwise has to be met by costly compensation payment.

Nevertheless, the current situation in Austria is making a successful transfer of findings complicated, as the organizational structure (BIG - universities government) is likely to cause friction between stakeholders instead of serving public interests. In order to take full advantage of a potential efficiency increase through space management, the government would have to work over the organizational structures and processes. But this is not the topic of this thesis.

Finally it needs to be mentioned, that, triggered by the (Austrian) interpretation of the Bologna process, teaching and studies at Austrian universities are becoming increasingly regimented (school like). While a strict organization clearly also bears advantages, it has a negative impact when trying to improve things through reorganization. E.g. temporal rearrangement of lectures could lead to a higher efficiency of space utilization, but with centralized, fixed schedules and/or curricula re-scheduling is not possible.

For objectivity reasons it has to pointed out, that, in order to enable smooth and uninterrupted studies, courses have to be scheduled - at least within branches of study - in such a way that there are no, or as little as possible, conflicting or overlapping teaching units. Such a schedule can of course be obtained much easier if coordinated centrally. Further such a schedule has the same effect on flexibility as the above mentioned regulation. 


\section{Chapter 2}

\section{From Model to Decision}

\subsection{Modeling and Simulation}

In the previous two sections of chapter 1 the focus was set upon the thesis' initial motivation and target for improvement. In this section the means used to achieve this aim - modeling and simulation - as well as its application will be explored.

Starting with a general overview on modeling and simulation the reasons for harnessing this approach will be outlined, together with a brief sketch of the diversity of the terminology. In the second section the concept of dynamic models will be analyzed and defined. Next the classical outline for simulation projects, the simulation process, will be expanded in order to allow for a holistic system analysis and thus avoid some of the most common problems.

\section{The Problem with the Terms}

The following two - unscientific - examples are to show the problem faced when trying to pin down the meaning of the term model - a search-engine check and a probe of Wikipedia.

The web-search for the term "model" produces $3.3 * 10^{9}$ hits with the search engine "Google", $9 * 10^{8}$ with "Yahoo" and $6.6 * 10^{8}$ with "Bing". On WikiPEDiA the English version offers three major categories for the term: "physical model", "nonphysical model" and "titles and names". Even by neglecting the last category, impressive 47 entries remain in the first two ${ }^{1}$. This extreme ambiguity induces a huge potential of misunderstanding for the word model and subsequently also for modeling.

Without providing any linguistic or etymologic proof, it does seem logical that the meanings of the term simulation, which implies the use or the existence of some kind of model, will vary depending on the (underlying) model. It can be assumed that such polysemy of the terms model, modeling and simulation poses an even bigger threat of confusion, misinterpretation or even disagreement if used in an interdisciplinary environment, and can thus subsequently if the worst comes to the worst - be a reason for (project) failures. To avoid

\footnotetext{
${ }^{1}$ Both, the web- and Wikipedia-search, were conducted on July 20, 2011.
} 
such confusion the capabilities of different types of models have to be precisely specified in (interdisciplinary) projects.

Within this section the focus will be set on mathematical and computational models ${ }^{2}$. As will be shown even with this reduction of the model scope there is still a wide range of different models and modeling approaches.

\subsubsection{General overview}

\section{Reasons for Simulation}

The reasons to use simulation are manifold. Most of the time models are used to reproduce (real world) systems, with their characteristics and behavior, and exploit them within a controllable environment. In such an environment simulation (experimentation) with these models allows studying the behavior of the (modeled and usually simplified) systems as well as testing assumptions and/or alternatives (What would happen if...?) on them. This generates information which can be condensed into understanding and knowledge of the (modeled) system and subsequently transferred back to the original system.

Often the use of a model (of the real system) is the only possible way to conduct an experiment - the reasons for this are as manifold as those for using simulation in the first place. Real world systems might, for example, be evolving too slowly in order to observe relevant characteristics (e.g. the development of the universe), or it might be unethical to conduct certain experiments (tests of nuclear weapons or medical drugs on humans). Sometimes it might simply be too expensive to run experiments "for real" (crash-tests) and/or too risky (training of pilots, medical staff or workers of nuclear power plants). The need to "extrapolate" systems in order to obtain information for planning and decision making is yet another reason (e.g. weather forecast, epidemiological models, etc.). While this listing is far from complete and could easily be prolonged, it does hint the idea of reasons for using simulation ${ }^{3}$.

On the other hand modeling also has shortcomings which need to be kept in mind. The basic and most relevant one being that any model is a simplification of the real system. Thus one of the main tasks when creating a model is the identification of relevant influencing factors and their appropriate "translation" within the model. Further, creating a model of a complex system is not a task solved within a few days or weeks. The necessary analysis of the original system, gathering of (input-) data and information for the creation of the model, adjustments, parameter identification, and so forth is a lengthy and complex process itself — and hence a costly one.

\section{Models and Approaches}

Regardless of the surrounding processes and tasks, the core of a simulation project remains the development of an adequate model. For this numerous different modeling approaches and techniques are available, all with respective strengths and weaknesses. Which approach is best used strongly depends on the system to be modeled. It is not necessary that there is only one suitable

\footnotetext{
${ }^{2}$ With the introduction of Business Process Models (BPM), at a later point, not all of the models within this thesis will be of such a nature.

${ }^{3}$ Further and other examples can be found in almost any general book on modeling and simulation such as, for example, Bungartz et al. (2009) and Sauerbier (1999).
} 
technique. In fact sometimes even a combination of two or more approaches might be needed, e.g. to overcome flaws of one method through a second ${ }^{4}$. Such models, utilizing a combination of approaches, are referred to as hybrid models.

Other possible classifications which distinguish simulation techniques according to their characteristics are

- discrete/continuous,

- deterministic/stochastic,

- bottom-up/top-down and

- dynamic/static.

Each two groups of these four categories are disjoint - the categories themselves are not. Thus a model(ing approach) can, as an example, potentially be discrete, bottom-up, stochastic and dynamic. Cellular automata (with stochastic rulesets) are such a modeling technique.

Assignment of a model to a group of the first three categories is straight forward and self-explanatory. If a model bears stochastic elements it is referred to as stochastic; if a model is based on discrete time (e.g. time is not continuous, updates are taking place only at specific time-steps without changes in between them) it is discrete. Approaches describing the system as a whole (e.g. differential equations for epidemics) are classified as top-down while those that describe the system's behavior via its "smallest entity" (e.g. agent-based models) are classified bottom-up.

Unfortunately this is not the case with the last category; distinguishing between dynamic and static models is an area of frequent dispute. The main reason for this is the lack of a commonly accepted definition of dynamic models, which is partly owed to the fact that the terms model and simulation themselves are extremely ambiguous. To clarify which characteristics a dynamic model needs to hold in terms of mathematical modeling and simulation, a technical definition will be set up in the following section.

\subsubsection{Defining Dynamic Modeling}

\section{Time-dependent Models}

The term dynamic is very frequently used in combination with systems or models, which is also stated in Sauerbier (1999), nevertheless there is no definition of a dynamic model, system or simulation or of its opposite (static model, system or simulation ${ }^{5}$ ). The general definition of simulation by Banks (1998),

"Simulation is the imitation of the operation of a real-world process or system over time. Simulation involves the generation of an artificial history of the system and the observation of that artificial history to draw inferences concerning the operation characteristics of the real system that is represented. Simulation is an indispensable problem solving methodology for the solution of many real-world

\footnotetext{
${ }^{4}$ For a detailed description of such an example see Emrich (2007), where a combination of two bottom-up methods (Cellular Automata and Agent-Based Modeling) is used to create an influenza-model for heterogeneous, urban populations.

${ }^{5}$ In the following model shall be used as a substitute for all three terms.
} 
problems. Simulation is used to describe and analyze the behavior of a system, ask what-if questions about the real system, and aid in the design of real systems. Both existing and conceptual systems can be modeled with simulation." (page 3-4),

introduces one core element necessary for distinction between dynamic and static models — the imitation of a process or system over time. Nonetheless it frequently happens that the concept of time is neglected when modeling. Following Fishwick (2007b) the three reasons for this are ...

"Number one: the underlying phenomenon being modeled is not being well understood. Number two: The phenomenon is well understood but the model takes too long to analyze or to run. Or number three: the model is more complex than its static equivalent."

Reason number three implies that static models can sometimes be used as an alternative to dynamic ones. Nevertheless, for the purposes of the present context, the element of time is necessary for a model to be referred to as dynamic model.

\section{Complexity of Systems}

The next aspect necessary for this thesis' definition is more or less obvious. A system which consists of only one variable or entity can be described by a simple (linear or nonlinear) function. Even though the mathematical function itself may to some degree be "complex", the underlying system itself is lacking complexity.

E.g. the costs $C$ for production of a product are the sum of a piece independent costs $c_{\text {base }}$ (factory heating, personnel, etc.) and the piece dependent costs $c_{\text {piece }}$ for raw materials which - because of economies of scale - do not increase in a linear but in a logarithmic way in dependence of the number of produced items $x$. Thus we can describe the model with the simple function

$$
C(x)=c_{\text {base }}+\log \left(c_{\text {piece }} * x\right)
$$

with two parameters (the fixed and piece-dependent costs) and only one variable $x$ - the number of pieces produced.

While such a model for the prediction/calculation of production costs does effectively fulfill its purpose, it shall not be considered dynamic, as it is lacking complexity from the modeling perspective - one self-referencing variable.

Thus the second condition of the definition of a dynamic model is the existence of at least two variables or entities within the system, which is also implied by the following definition of simulation.

\section{Interacting Processes}

Even though this second definition, as found in Verein Deutscher Ingenieure (1996), is being a "technical" one ${ }^{6}$ it still remains very general:

\footnotetext{
${ }^{6}$ The "Verein Deutscher Ingenieure" (VDI) is the Association of German Engineers. Its role is comparable to that of the American Society of Civil Engineers (ASCE).
} 
"Simulation ist ein Verfahren zur Nachbildung eines $\rightarrow$ Systems mit seinen dynamischen Prozessen in einem experimentierbaren $\rightarrow$ Modell, um zu Erkenntnissen zu gelangen, die auf die Wirklichkeit übertragber sind.

Im weiteren Sinne wird unter Simulation das Vorbereiten, Durchführen und Auswerten gezielter $\rightarrow$ Experimente mit einem Simulationsmodell verstanden.

Mit Hilfe der Simulation kann das zeitliche Ablaufverhalten komplexer Systeme untersucht werden (s. a. $\rightarrow$ Simulationsmethode)."

Where " $\rightarrow$ " denotes cross references to other definitions within Verein Deutscher Ingenieure (1996). When translated from German into English, this reads as:

"Simulation is a method to reproduce a $\rightarrow$ system with its dynamic processes within a $\rightarrow$ model suitable for experimentation, in order to gain insights that can be transferred to reality.

In a broader sense simulation can be understood as the preparation, operation and evaluation of specific $\rightarrow$ experiments with a simulation model.

The aid of simulation allows for examination of the temporal kind of flow of complex systems (see $\rightarrow$ simulation method)."

Nevertheless, when compared to the first definition of Banks (1998), it does introduce a third very important aspect - the reproduction of a system with its (inherent) dynamic processes. Which also does back the second aspect of multiple variables/entities, in this case referred to as "processes". Following this definition "dynamic" does not refer to the system itself. Rather a dynamic model is characterized as such by the processes contained.

With this aspect in mind, the example of a model for the production costs from page 24 can again be regarded as static ${ }^{7}$. It is completely described by function 2.1, which is not a dynamic process (but a linear function).

Subsequently the question is how a dynamic process is being defined. As the model has to evolve over time (as described in section 2.1.2) and contain more than one variable/entity (section 2.1.2) a sensible approach is to request that the models variables/entities (and thus the processes describing these) are interlinked in such a way that their changes (can) cause interactions. One of the most prominent examples for such interactions are feedback loops.

\section{Definition of Dynamic Models}

Summarizing the three aspects described above, throughout this thesis ${ }^{8}$ a model shall be considered dynamic only if it fulfills following criteria:

1. The model evolves over time (time-dependence)

\footnotetext{
${ }^{7}$ Where static denotes the opposite of dynamic.

${ }^{8}$ The reader is kindly invited to use this definition for her purposes. Due to the numerous perspectives and definitions of "modeling" and "simulation" this definition does not claim universal validity.
} 
2. The model consists of more than one variable/entities (sufficient complexity)

3. The variables/entities are somehow connected and influence each other (interacting processes)

Nevertheless the definition still leaves a high degree of freedom. E.g. role play exercises can be regarded as dynamic simulations with respect to it. In order to narrow this down further, in this thesis "simulation" will refer to computer simulation only. This stands for simulations carried out on computers or, to be more precise, by computer programs.

For a detailed general introduction to computer simulation, reference is made to McHaney (1991). Further reference, for a very thorough overview of dynamic simulation (satisfying above definition), is made to Fishwick (2007a). And for a general introduction to the spectrum of (mathematical) concepts used in simulation - from discrete structures, continuous system (linear algebra and analysis), stochastic and statistical foundations to numerical methods - to Bungartz et al. (2009).

\subsubsection{The Simulation Process}

As indicated before, development of a (valid) simulation model and experimentation with it, are usually "only" the core tasks of a simulation project. In order for the whole project to be a success further steps and tasks are necessary. Thus this section will analyze the simulation process with a focus on the tasks surrounding the "core" - starting with a comparison of process models ${ }^{9}$ found in classical modeling and simulation literature. Based on these findings the definition will be broadened and expanded so that it better reflects the holistic perspective necessary for many complex projects.

\section{The Classical Definition}

Even though the details vary throughout classical (modeling) literature, simulation projects are usually described as processes that start with a given problem statement or the assignment to create a specific simulation model. Next the requirements for this task are analyzed (data, technical system, etc.), followed by formalization of a model, implementation and its verification and validation. Finally the model is used to conduct experiments, producing results which are then interpreted.

A comparison of such process models is made by Rabe et al. (2008, see chapter 3). Rabe et al. also propose a process model of their own, which is shown in Fig. 2.1 and also sticks to the pattern described above.

Some descriptions, such as by Bungartz et al. (2009), who refer to their process description as simulation pipeline, also include embedding/integration of the model. But this term refers to the procedure of embedding software interfaces with the surrounding system (see Fig. 2.2).

Although catchy, the term pipeline and to some extent the image in Fig. 2.2 as well, are misleading as they imply a straight-forward process - which seldom

\footnotetext{
${ }^{9}$ The process model itself is not a mathematical simulation model but, as explained in section 2.1 (ambiguity), denoting a different kind of model, namely one depicting the process of creating and using a simulation model.
} 


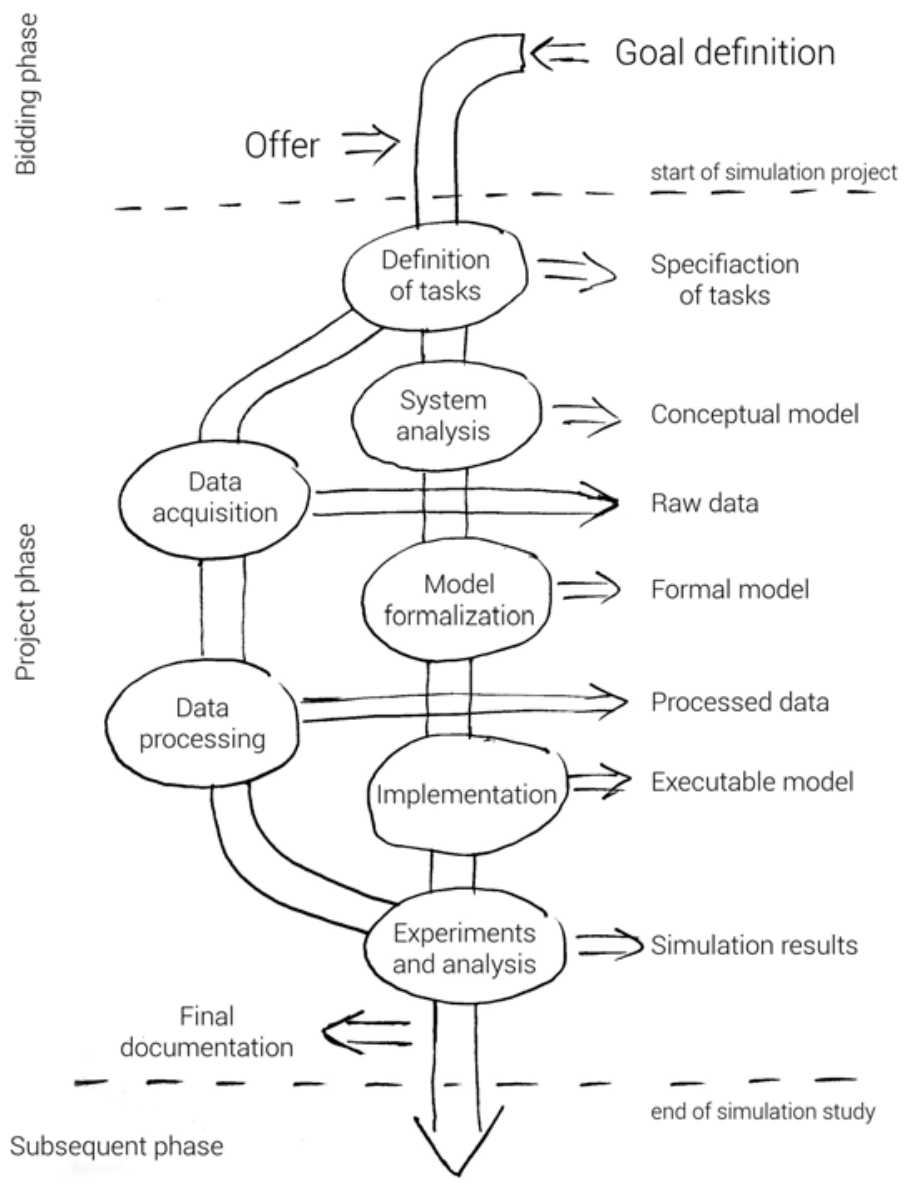

Figure 2.1: Process model as described by Rabe et al. (2008) who also conducted a comparison of process models in classical modeling literature.

is the case. Creation of a model usually is an iterative (and looped) process of system analysis, implementation, validation and parameter identification ${ }^{10}$.

In general, one can identify three task-clusters. The exact borders between them vary, depending on the definition used or the process model considered. The numbering is primarily to distinguish among the parts, especially as the nature of the process is, as pointed out, an iterative one.

1. System analysis and modeling identification of the main influencing factors and setting up of the model

2. Verification, validation and parametrization of the model testing of the model's validity

3. Utilization of/experimentation with the model

\footnotetext{
${ }^{10}$ In Fig. 2.2 the iterative nature of the process is hinted at by the inside arrows pointing from "validation" to the left.
} 


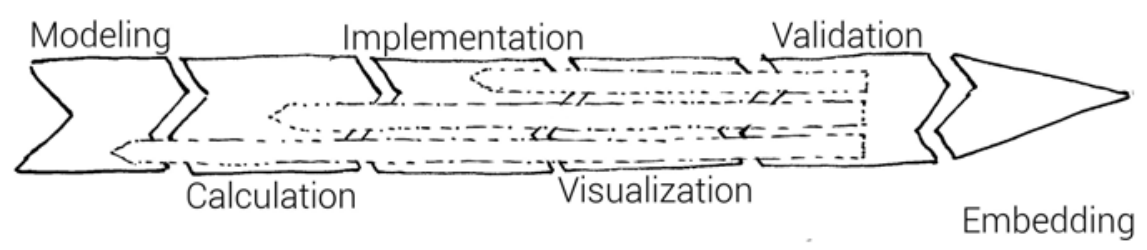

Figure 2.2: So-called simulation pipeline of a simulation process.

While such process models are intended to solve the technical problems posed, they generally neglect a holistic system analysis and the embedding of the created model within the surrounding system - in terms of process-interfaces and decision making. Depending on the type of project these two aspects might not be of highest priority. Still, often it is them that decide upon the outcome of a project. For example is might be sufficient to find an answer to a single question (e.g. where is the safest position for an airbag inside a car). But often simulation models are to be harnessed on a daily or even hourly basis (e.g. weather forecast for air traffic control). In the latter case not only will it be necessary for the simulation model to provide correct forecasts, but also that these results reach the right people in an adequate form. To stick with the example of air traffic control, the head of the team will most likely not have time to run over an hourly 20 sheets of paper full with data points for a 50 kilometer radius around an airport. Instead she might want a map indicating the situation with some general data, highlighting critical factors.

\section{Additional Considerations}

As the previous air traffic control example indirectly illustrates, it is not possible to adjudicate on a solution (obtained by a simulation model) without knowing the goal of the model or the intention for its use. This insight is crucial as it adjusts the focus - away from the model and onto the purpose for modeling! A successful project needs clearly formulated goal-definitions, a "question to answer".

It can be assumed that this definition is preceded by identification of some kind of shortcoming or problem within the given system. Upon this the decision maker(s) in charge come(s) up with a strategy to improve the situation - in this case the initiation of a simulation project. At this point the classical definition of the simulation process sets off with the assignment to create a model to simulate the given system. In many projects this hand-off, setting the project's course at an early stage, is a major contributing factor to its success - or failure.

One may argue that it is the job of the responsible decision makers to clearly set out the direction and the goal(s) of the project, but in the end the outcome counts. It determines which impression the professions involved will have from each other. Thus, in order to improve the reputation of modeling and simulation in general - and one's own in particular - this hand-over has to receive special attention. Also it has to be kept in mind that the ordering party, in most cases, will not be a modeling and simulation expert and thus with only limited knowledge in this field.

In order to reduce the chance of setting the project out on a wrong path, 
the system analysis and definition of goals should be done cooperatively by the ordering party and the simulation experts. This requires a common language as well as - at least - basic understanding of the work scope of the other ${ }^{11}$.

The same holds true for the utilization of the finished model if it is to be used on a regular basis. In such a case the model has to be integrated into the system's daily processes in order to obtain necessary (input) data and parameters and also for proper dissemination of the results produced. Ideally one will understand which results have to be communicated how and to who through the insight gained during system analysis (conducted at the beginning of the project). Thus this (final) step of the project is based upon the first one.

\section{An Expanded Definition}

To formalize this expanded understanding of the simulation process the three steps of the "classical" definition are merged with the problem solution process proposed by Schalcher (2008) and Ulrich and Probst (1991), depicted in Fig. 2.3. Comparing this process to the three "classical" steps it becomes understandable that these make up only the very core of the "modeling and interpretation" section, although with fuzzy borders. One is tempted to heretical argue that the classical simulation process is starting somewhere along the question "what is the problem situation?" and ending halfway through "how can we affect the situation?". The system analysis' approach to problem solving perceives the task in a much broader way. Yet it is noteworthy that in this broader perspective, even though at a very different level, a clear separation between decision making and modeling remains.

Summing up, the extended process can be laid out in five phases, starting with the analysis of the system, which is - performed in a holistic way - regarded as a separate phase and placed before the modeling. Then the three classical steps (the first one being stripped-down) follow. Embedding of the model, respectively its integration within the system poses the final phase. Decision making and actions derived are not a part of the simulation process, but are listed for the sake of completeness.

- Initiation of process

Identification of a potential for improvement or necessity to change; definition of project's aim/goal(s).

1. System analysis

A holistic analysis of the system (not only of technical aspects) its boundaries and the relevant environment. Such a view is, according to Schalcher (2008) only possible "if the interdependence of all human activity and natural events is consciously accepted and not neglected". This step should also (re-)evaluate the goal-definitions of the project, as previously described.

2. Modeling

Development of the model including its algorithmic framework and the implementation in a programming language.

\footnotetext{
${ }^{11}$ The ordering party needs to understand the basics of simulation while the simulation experts need to have a (fairly solid) know-how of the business they are about to model.
} 


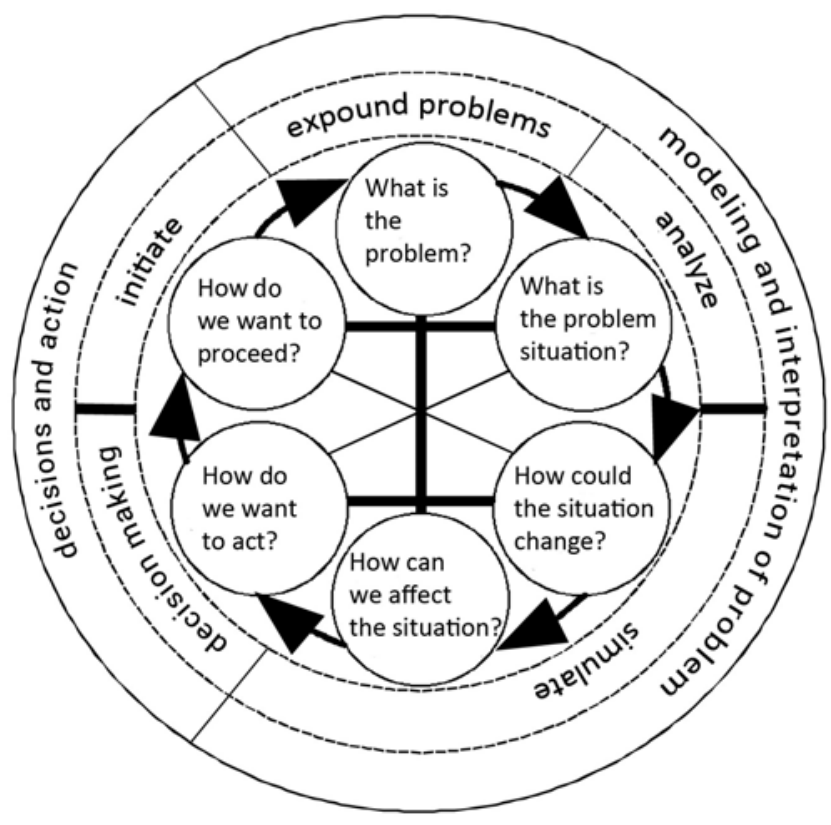

Figure 2.3: Problem solution process with initiation and decision making (source: Ulrich and Probst (1991)).

3. Verification, validation and parametrization Thorough testing of the model regarding its algorithmic correctness and its sensibility (representation of the system's behavior), as well as the tuning of the necessary input-parameters.

4. Utilization of the model

Conducting experiments and tests with the model, interpretation of the obtained results ${ }^{12}$.

5. Embedding the model within its surrounding system

This stage is not necessarily part of every simulation project. But if integration of the model is required, it is necessary to not only provide the right (software) interfaces but also to deliver meaningful results to the appropriate stakeholders.

- Decision making

Deriving actions from the information gained.

As previously argued, this process is of an iterative nature. Especially with the boarders between the steps being fuzzy, and even more so with their order being subject to permutation. This is best explained with step "embedding of the model", which is strongly depending on the findings of system analysis, running in parallel to modeling, and taking place prior to and after utilization

\footnotetext{
${ }^{12}$ Information visualization is a very powerful way to derive information from model results and to support decision makers. Nevertheless the task of visualizing can be regarded as part of model implementation, utilization, the embedding procedure or a combination of these. Therefore it is not explicitly included in this listing.
} 
of the model. Thus the numbering is primarily for identify of the phases and to point out the difference between the approaches.

The methods used for and within the newly added phases (i.e. system analysis and model integration) will be laid out in chapter 3 , along with a description of the mathematical modeling concepts used. However the two stages surrounding the (expanded) simulation process, initiation of the process and decision making based upon simulation results, will not be covered in this thesis.

The reason for the former is that decisions to start a simulation project will most of the time be taken within an institution or company - for manifold reasons. Potential triggers can involve identification of bottlenecks or of an improvement-potential. Others might be upcoming changes for which one needs or wants to prepare. An example for the latter would be the strong increase in student numbers due to a change in Germany's educational system ${ }^{13}$, which lead two consecutive age groups to enter universities together in 2011. Frequently data mining or analysis of the systems will be the base for such decisions. An analysis of these aspects would lead to far from the present topic.

The same applies for the second phase - dissemination of project results. In this case because the transfer of the outcome of a simulation project and decision making based upon it, especially implementation of structural or organizational changes, are themselves highly complex processes. Often sophisticated change management will be required, as diverse aspects (e.g. psychological aspects, such as employee satisfaction) need to be taken care of. In addition, at (Austrian) universities ${ }^{14}$ such changes will result in institution-internal political controversy. However the aspects and problems which are caused by this will be described later (see section 4.1.3 - "Problems Encountered").

\footnotetext{
${ }^{13}$ High school was reduced from 13 levels of education to 12 in several federal states.

${ }^{14}$ And other institutions with a similarly weak central management and highly independent staff.
} 


\subsection{Towards the problem's solution}

\subsubsection{Mathematical Optimization}

\section{Mathematical Definition}

In a mathematical context the term optimization denotes the process of finding the best solution for a given problem - the optimum. Although this might not seem that intriguing, the meaning is fairly different from the colloquial use of the term, which usually implies plain improvement — without ambition of finding the true maximum (or minimum).

Depending on the nature of the problem, the optimum may be a single solution or a set of (equally good) solutions. Further, a distinction is made between local and global optima. A local optimum is, as its name implies, the best solution within a given neighborhood, while a global optimum is the best solution over the whole problem(range).

Analytically speaking, the search for a problems optimum is equivalent to that of finding the problem's objective function's extreme values. Thereby it is regardless if finding the optimum means to search for a minimum or a maximum, as a simple multiplication of the objective function by -1 turns a minimum into a maximum and vice versa. Given the (objective) function

$$
f(x)=x^{3}-x
$$

(see also Fig. 2.4) the extreme values are calculated as finding the roots of the function's derivative $f^{\prime}(x)=3 x^{2}-1$. Solving this quadratic equation with the quadratic formula

$$
x_{1,2}=\frac{-b \pm \sqrt{b^{2}-4 a c}}{2 a}
$$

where the letters $a, b$ and $c$ denote the coefficients of the terms as in

$$
a * x^{2}+b * x+c=0,
$$

produces the two zeroes $\left(x_{1}\right.$ and $\left.x_{2}\right)$

$$
\begin{aligned}
x_{1,2} & =\frac{0 \pm \sqrt{0-4 * 3 *(-1)}}{2 * 3}=\frac{ \pm \sqrt{12}}{6} \\
& =\frac{ \pm 2 * \sqrt{3}}{6}=\frac{ \pm \sqrt{3}}{3} \\
& =\frac{ \pm \sqrt{3}}{\sqrt{3} * \sqrt{3}}= \pm \frac{1}{\sqrt{3}} \\
x_{1} & =+\frac{1}{\sqrt{3}} \\
x_{2} & =-\frac{1}{\sqrt{3}} .
\end{aligned}
$$

Optimization of a problem with objective function described by equation (2.2), where the task is to find the minimum $\min _{x \in I} f(x)$ within the interval $I=[-1, \infty)$ would thus produce the optimum $x=1 / \sqrt{3}$, whereas the search for the maximum $\max _{x \in I} f(x)$ would yield $x=+\infty$. 


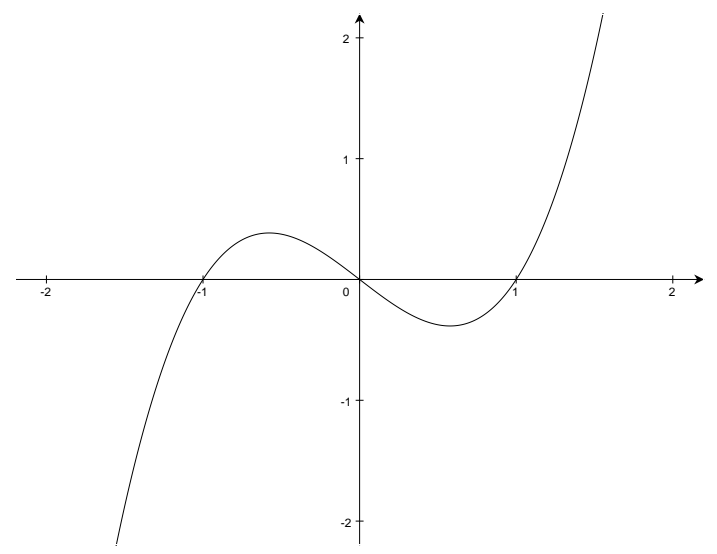

Figure 2.4: Plot of cubic function $f(x)=x^{3}-x$ with extreme values (local minimum and maximum at $\left.x= \pm \frac{1}{\sqrt{3}}\right)$.

\section{Multi-Objective Optimization and Pareto Efficiency}

Real world optimization problems are usually not as simple as above case, in which the objective function depends on a single variable. Often the objective function will be subject to constraints that have to be satisfied and/or incorporate more variables. Following example of a factory producing T-shirts and boxer shorts shall illustrate the problems faced.

The target function for this company shall be the net profit generated through sale of the products. The company is selling T-shirts (denoted as $x$ ) for $\$ 2.50$ and boxer shorts $(y)$ for $\$ 4.00$ a piece. Formally the optimization problem is to maximize the profit $(p)$, which obeys the objective function

$$
p=2.5 x+4 y .
$$

With this information alone the company should specialize on producing Tshirts as theses yield more revenue. But following constraints need to be taken into account. The company has a fixed contract for fabric and receives 500 units of it per day. Working power is also limited, as the company employs 5 people who work for 8 hours/day. This means that labor time is limited to 40 hours per day. Production wise T-shirts require 5 units of fabric and a working time of $1 / 4$ hour and boxer shorts 2 units of fabric and $1 / 2$ hour. And finally, the production of boxers is limited to at most 60 pieces (e.g. due to limited button supply). Formally these restrictions are the side conditions

$$
\begin{aligned}
5 x+2 y & \leq 500 \\
0.25 x+0.5 y & \leq 40 \\
y & \leq 60
\end{aligned}
$$

Of course the produced number of shirts $(x)$ and boxers $(y)$ cannot be negative and as such $x \geq 0$ and $y \geq 0$ have to be added to the above set, leading to the optimization problem depicted in Fig. 2.5.

Such problems are usually solved using linear programming. In the given (simple) example this can also be achieved via its graphic representation, as 


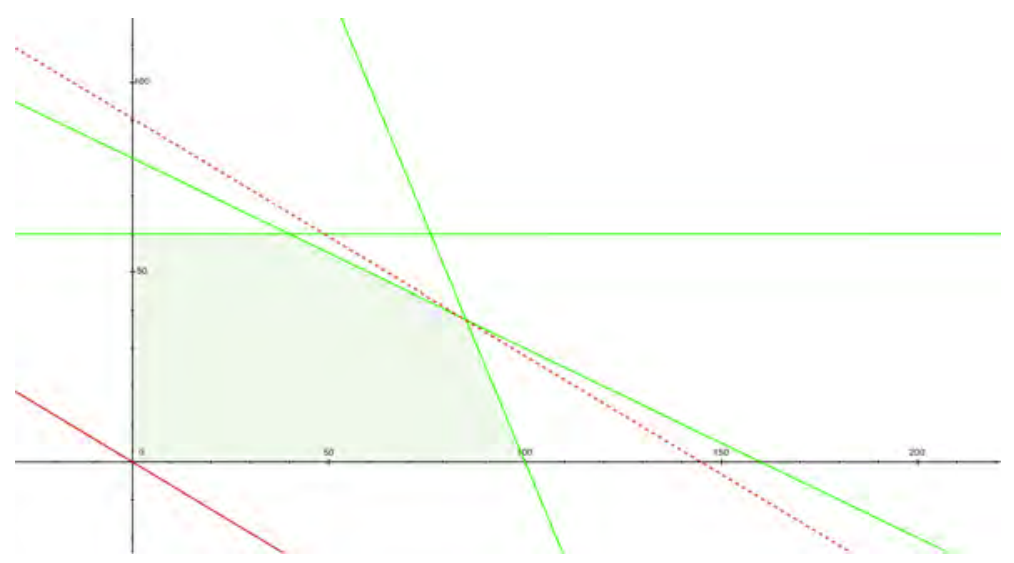

Figure 2.5: Linear programming problem for a factory producing two products; boundary conditions (equations 2.6) in green, resulting solution space in light green, objective function (equation 2.5) in solid red and its parallel translation dashed red.

the maximum revenue is generated at the intersection of the side conditions $y \leq 80-x / 2$ and $y \leq 250-5 / 2 x$ which is found by solving the equation $80-x / 2=250-5 / 2 x$. The solution yielding the maximum profit is thus located in the point $x=85$ and $y=37.5$, which means production of 85 T-shirts and 37.5 boxers per day.

As soon as there are more than two variables involved (e.g. jeans being the third product produced in the factory) one would have to use a three dimensional graph in order to visually/manually solve the problem - which is not practical any more. In such cases algorithmic solutions (e.g. utilization of the simplex algorithm) become inevitable.

So far the above example is aiming only at optimizing the clothing factory's profit. Staying with this case, things start to look differently when a second, conflicting criterion is added to the optimization problem; greenhouse gas (GHG) emissions for example. Solutions producing as little GHG emissions as possible are favored over those with high emissions.

Theoretically it is possible to convert emission into currency via $\mathrm{CO}_{2}$ certificates $^{15}$. In such a way emissions could be incorporated within the objective function by calculating the emissions of a single shirt or boxer short respectively and then reduce the products revenue value by the emissions monetary value. Another example would be to add the maximization of employee satisfaction as a second optimization goal. Here it is already a challenge to even measure the (soft) objective itself. Afterwards it might again be possible to somehow evaluate employees satisfaction in monetary terms (e.g. via staff sick days, productivity, innovativeness, etc.), but again only to a limited extent.

Even though the above - contradictory - new objectives can be somehow linked to the initial objective (obtaining maximum revenue) so that they become comparable, this does not provide a solution to the problem to optimize both objectives. In order to find a solution for the optimization problem (with contradictory objectives) it is necessary to find points which provide improved

\footnotetext{
${ }^{15}$ Practically $\mathrm{CO}_{2}$ certificates are not yet at a stage where they truly represent the impact of emissions.
} 
solutions with respect to every objective. As the objectives are of contradictory nature, one will come across the point from where on improvement in one objectives direction leads to a worsening in that of another. Such points are called Pareto optima or Pareto efficient points ${ }^{16}$. The set of all Pareto optimal points is referred to as Pareto frontier.

So it exists, the optimum solution in the example with one objective and two variables (as depicted in Fig. 2.5 is either a single point or a line ${ }^{17}$. This changes when a second objective is added, now there is a solution-set for every criterion. These sets have to be combined within a "meta-solution", forming the problems Pareto frontier (which can be two-, three- or multi-dimensional). For the sake of simplicity the implications of such a Pareto-frontier shall be explained using a two-dimensional graph.

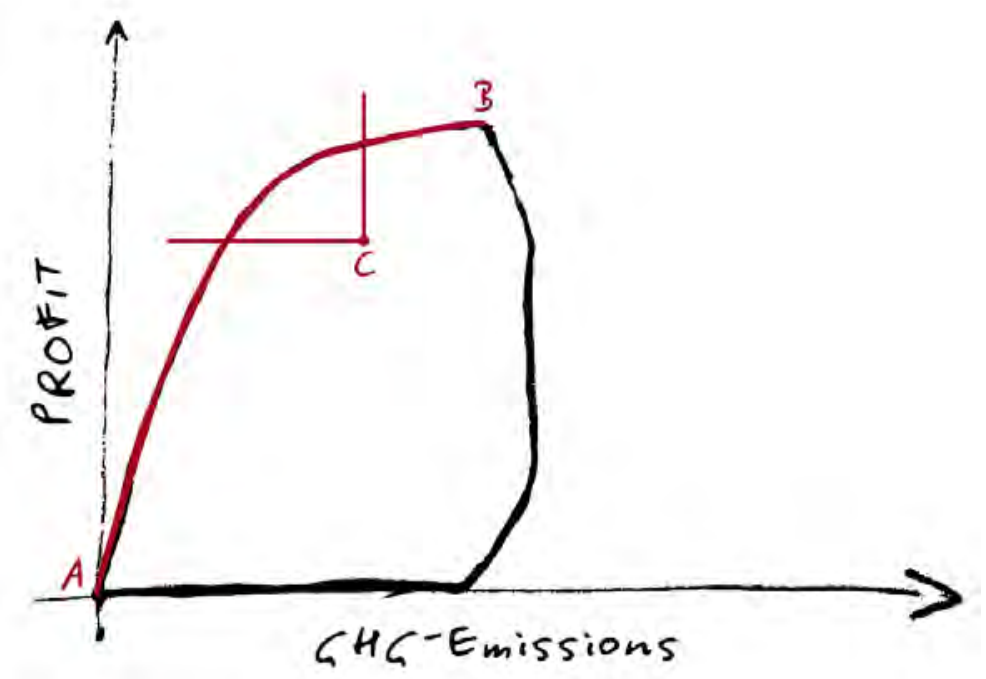

Figure 2.6: Value set for two contradictory objectives ("profit" and "GHG-emissions") with its Pareto frontier (from $A$ to $B$ ) marked in red and the "pie slice" for all superior points of $C$.

In Fig. 2.6, which shows the value set for the objectives "profit" (maximize) and "GHG emissions" (minimize), the Pareto frontier is highlighted red (from point $A$ to $B$ ). From point $A$ towards $B$ the GHG emissions are increasing while the profit rises. These points cannot be compared to each other as they are Pareto optimal. It is not possible to obtain a better solution with respect to both objectives.

Following the set's frontier beyond $B$, emissions increase while profit decreases. Thus these solutions are inferior to point $B$ with respect to both criteria. The decrease settles in a steady vertical slope at the far right of the set where emissions do not increase further, but the profit still declines (inferior to $\mathrm{B}$ in both objectives). After this slope emissions and profit decrease until the profit hits 0 (inferior to $B$ with respect to profit). The edge of the set continues

\footnotetext{
${ }^{16}$ Named after Vilfredo Pareto, an Italian economist.

${ }^{17}$ While this resembles a theoretically infinite set of points, it might practically be a countable or finite set, as some problems might only accept discrete solutions.
} 
to follow the $\mathrm{x}$-axis decreasing towards zero, where it reaches point $A$. Until reaching $A$, all points are having a "dual point" with equal x-value and higher $\mathrm{y}$-value on the Pareto-frontier and are thus inferior to these.

All other (internal) points of the value set - for which point C (in Fig. 2.6) is a representative - are not Pareto efficient as there are superior solutions with respect to both objectives. I.e. all points within the "pie slice" defined by the internal point (as lower right corner of the slice), the horizontal and vertical lines through the point and the "northern" edge of the value set (see Fig. 2.6 with pie slice of $C){ }^{18}$

\subsubsection{Finding Good Enough Solutions}

The goals within multi-objective optimization problems are, as shown above, often contradictory. Thus, in order to come up with a solution at all, it is necessary to understand and accept its formally imperfect nature. It is the task to find a compromise that satisfies all formal constraints and that is the most favorable for the involved decision makers. Or that one with the least negative impact, respectively. These decision makers have to accept and choose a trade off in one direction or another, an arbitrary weighting of objectives.

\section{Manual and Automatic Approaches}

This weighting can be carried out either automatically or manually. For the manual process a procedure (algorithm, simulation, etc.), that produces a set of feasible solutions from which a decision maker then chooses the one solution that she ranks best, is required. As described in Das and Dennis (1996) this approach makes it necessary that the set of solutions is presented in an understandable/readable manner to the decision makers, usually via some sort of visualization. And, as they put it, this "visualization process may be complicated for more than three objectives, and how helpful it will be in guiding the user towards a better choice may depend on factors like the psychological aspects of the visualization". The automated process, on the other hand, requires that the objective-weighting is already implemented within the problem-solving algorithm, which can be achieved in several different ways.

The weighting can either be incorporated into the objective function, leading to the construction of a single aggregate objective function (AOF), as for example described by Messac et al. (2000). This approach is arguably the most intuitive. In order to solve problem, it is required, as the name indicates, to find the (best) solution of a single function, the AOF. Alternatively, the objective functions can be computed independently and their respective solutions subsequently used as input of a weighting function. In any case the weighting (inside the AOF or within the "external" weighting function) again requires a decision maker's assessment. Because of the important role within the weighting process, the decision maker has to be carefully selected, in terms of her competence of the problem as well as her hierarchical position.

\footnotetext{
${ }^{18}$ The here sketched boundaries are of course for the present optimization problem. If the objective-directions change (e.g. maximization of both) the location of the Pareto frontier will change as well.
} 


\section{Optima and Sufficient Solutions}

Following equation (composed of several, fairly simple trigonometric components) is to illustrate the problems of finding the (local) optimum of an unsteady - even though periodical - objective function:

$$
\begin{aligned}
f(x, y)= & -0.2\left(\sin (2 x+y)-2 \cos \left(x^{2}+4 y\right)\right. \\
& \left.-3 \sin \left(3 x-y^{2}\right)+4 \cos (y)\right)
\end{aligned}
$$

It is obvious, that this equation is composed by only two variables, namely $x$ and $y$. Nevertheless, as visible in the plots shown in Fig. 2.7, this function is rich in (local) maxima, respectively minima ${ }^{19}$. Finding the global maximum - or maxima, if multiple exits - of such a rough function with non-analytic methods, is a hardship for any search-algorithm.

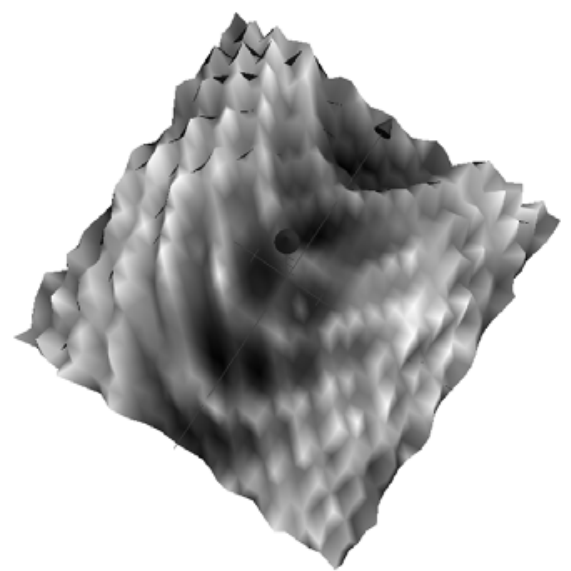

(a) Top view

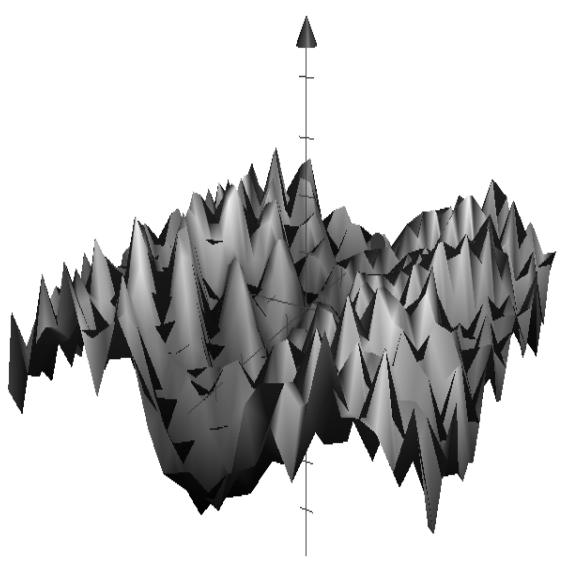

(b) Side view

Figure 2.7: Graph of the objective function described by equation (2.7), viewed from above (left) and from the side (right). The highest values of the function are coded white, the lowest black.

Because of its components' trigonometric nature, the function's maximum value is limited: $\cos (z)$, as well as $\sin (z)$, can be at most \pm 1 . Consequently the sum of the equation's four terms cannot exceed $\pm 1 \pm 2 \pm 3 \pm 4= \pm 10$. Multiplication of this theoretical extreme value by -0.2 leaves \pm 2 as upper, respectively lower bound of equation (2.7). In order to obtain this extreme value all trigonometric terms have to, simultaneously ${ }^{20}$, assume their extreme value. The inner terms of the sine functions would have to equal $\pm(4 k+1) * \pi / 2$

\footnotetext{
${ }^{19}$ In the interest of simplification the term "maximum" shall be used from here on, as the minimum is the maximum of the inverted function.

${ }^{20}$ Thus in one point $(x, y)$
} 
and those of the cosine functions $\pm 2 k \pi$ with $k \in \mathbb{Z}$. Thus the non-linear system

$$
\begin{aligned}
(4 k+1) * \frac{\pi}{2} & =2 x+y, \\
(4 k-1) * \frac{\pi}{2} & =3 x-y^{2}, \\
(2 k-1) * \pi & =x^{2}+4 y \text { and } \\
2 k \pi & =y
\end{aligned}
$$

has to be solved for $x$ and $y$, with $k \in \mathbb{Z}$.

From the respective sub-functions' repetitive behavior it may be assumed, that the given upper or lower bounds of the objective functions might be reached for sufficiently large (or small) values of $x$ and $y$, which is also illustrated in Fig. 2.8, especially with the quadratic functions' trajectories (defined by equations (2.9) and (2.10)) running closer and closer with increasing distance to the axis $^{21}$.

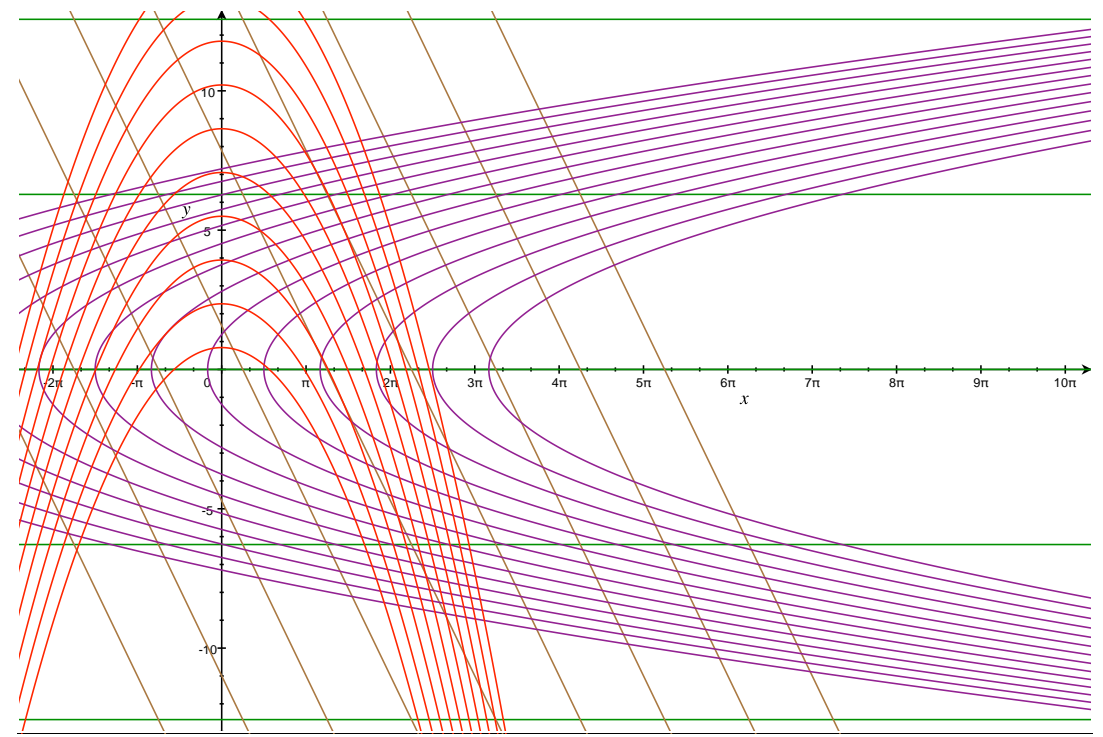

Figure 2.8: Plot of equations (2.8) - (2.11) (for some values of $k$ ), where an intersection of all four would mark a global maximum of objective function 2.7 .

If a problem cannot be solved analytically because of its nature (e.g. the objective function cannot be solved analytically or a simulation model is set up without an explicit mathematical function), alternative approaches have to be harnessed. Theses have to solve the problem step by step, searching for the best - or an acceptably good - solution, which has to be reached in reasonable time.

Scanning the whole search space, i.e. all possible input-combinations, will seldom be an option ${ }^{22}$, as the search space of complex problems usually exceeds

\footnotetext{
${ }^{21}$ For the sake of completeness, it is noted, that $f(x, y) \approx-1.9976$ for $(x, y)=(3.99,6.22)$, which is arguably close to the theoretical minimum of -2 , which can be obtained through Fig. 2.8. However, this is not the intended automated approach.

${ }_{22}^{2}$ If it is an option, it is referred to as "brute force" approach, as it relies on testing every combination without any "intelligence" behind. Comparable to a thief trying to open a bikelock by trying all its combinations (e.g. $0000--9999)$ and not picking the lock instead.
} 
the computational capabilities not only of today's computers, but also of those that will be built in years to come $^{23}$. Thus it is required to utilize an algorithm that relies on some kind of strategy in order to come up with a solution for the problem.

Generally such solutions will not represent the absolute optimum for a given problem. It has to be accepted that the produced result are "reasonably good" and not perfect. This of course, strongly depends on the problem and its sensitivity. In practice the quality of the solution will also depend on the available computing resources, or, viewed upon from the opposite perspective, on the efficiency of the implemented algorithms/models. Since it can be assumed that a user will buy an adequately strong computer (workstation, server, cluster, etc.) in order to perform the necessary optimization, the effectiveness of the algorithms/models will more often prove to be the limiting factor than the available hardware. This is especially true, when entering scales of problems such as global weather models or high-end simulation of molecular processes, which can be run only ${ }^{24}$ on today's supercomputers.

To sum things up, in order to solve such problems, it will be necessary to accept reasonably good solutions. For this it is necessary to utilize special algorithms, capable of "intelligently" scan the search space for such a solution.

\section{Metaheuristics, Evolutionary Algorithms \& Co.}

Metaheuristic approaches represent a frequently used set of techniques used for evaluation and iterative improvement of candidate solutions of a given problem. Some of the best known heuristic algorithms are inspired by natural processes, such as evolution (evolutionary or genetic algorithms), ant colonies (ant colony optimization) or swarms of animals/insects (particle swarm algorithms). But solidification of liquid materials during cooling has also inspired a family of algorithms (simulated annealing). For a very interesting and thorough introduction to complexity theory and metaheuristics, reference is given to Mitchell (2009).

In general such (meta-) heuristic approaches will not yield the optimal solution. Practically all existing algorithms are capable of finding local maxima of objective functions. The challenge, though, is to find the global maximum, or a solution sufficiently close to it, in reasonable run-time - which is a much harder task (compare to above example). Metaphorically speaking, finding a local optimum of an objective function such as defined by equation 2.7 simply requires to choose a random starting point and keep "walking" in the direction of improvement until no further improvement is possible. The point found is a local optimum. Finding the global optimum is equivalent to identification of the best of all local optima. This of course requires knowledge of all local optima in order to select the globally best. Even more so if the problem has several global optima (i.e. in the above case $f(x, y)= \pm 2$ ). While in some cases it might be sufficient to stop the algorithm after finding the first global optimum - which implies beforehand knowledge of the optima's value! - additional constraints might ask for more appropriately situated points with optimal solution.

\footnotetext{
${ }^{23}$ For the sake of completeness: yet to be developed quantum computers might be able to change this situation. Though this remains an unsettled dispute among computer scientists, as Bernstein and Vazirani (1997) show that the question cannot be answered.

${ }^{24}$ Nevertheless, simplification of the model or limitation of the problem (e.g. local instead behavior instead of global or smaller time intervals) may allow it for the problem to be solved also on "smaller" machines.
} 
Since complete knowledge of the objective function does not exist, metaheuristics can heretically be referred to as "educated guessing". Respective algorithms try to find reasonably good solutions improving a single or a set of randomly chosen starting points via strategies that often seem incomprehensible to the outsider. ${ }^{25}$ Nevertheless, for a lot of problems in various fields of applications the results obtained by metaheuristic approaches do produce satisfactory solutions. Or as Jason Lohn, a computer scientist working at NASA Ames Research Center put it in Williams (2005): "Evolutionary algorithms are a great tool for exploring the dark corners of design space. You show [your results] to people with 25 years' experience in the industry and they say, 'Wow, does that really work?'."

In the end, the decision whether to choose the manual path or an automated method, with or without the use of metaheuristics, to come up with a solution for a problem, will depend on a lot of influencing factors. It is impossible to answer this question in general, as the answer will vary from situation to situation and will be affected by the goals, the data, the problem's nature, the given system's structure, interactions and many more.

\subsubsection{Settling for Room Allocation Instead of Timetabling}

When setting the focus onto this thesis' aim - increasing the efficiency of room utilization at universities - the previously mentioned aspects need to be taken into account. In addition to these technical aspects, with universities being located in an intense social and socio-political area of conflict, as described in section 1.2.4, additional aspects have to be taken care of. In principle university room utilization is directly linked to university timetabling and the assignment of adequate "rooms", which may be laboratories as well as large auditoria or small seminar rooms, to the respective courses.

Hereby it must be kept in mind that the requirements towards timetabling/ scheduling in education vary widely, depending on the considered nations' or regions' educational systems and teaching forms. U.S. senior high school stu$\operatorname{dents}^{26}$, for example, are choosing their courses and thus create individual timetables. According to these they then move from one course to another, with the courses being held by the teachers in "their" rooms. The timetable is repeated every day of the week; the choosing of courses is subject to certain constraints (e.g. a minimum of mathematics courses are needed to obtain the high school degree). In the Austrian Oberstufe ${ }^{27}$, on the other hand, the schedule is planned centrally and for "classes" of students. These classes are age-consistent ${ }^{28}$ groups of students that are assigned a room which is visited by the respective teacher. Spare some special courses (e.g. physical education, chemistry, etc.) which are held in according rooms that are visited by both, students and teachers. Classes thus share a common timetable which is usually different for every day of the week. It is clear that timetabling approaches for a U.S. high school and for an Austrian Oberstufe have to follow different

\footnotetext{
${ }^{25}$ In Mitchell et al. (1996), Crutchfield et al. (1998) and Mitchell (2009) fascinating insight is given on approaches towards understand the evolutionary processes within such algorithms.

${ }^{26}$ Students in grades 9 trough 12.

${ }^{27}$ The Oberstufe is comparable to the level of education of senior high school in the U.S. consisting of grades 9 to 12 .

${ }^{28}$ Spare students that have failed or skipped a level of education.
} 
objectives and need to take care of different restraints.

\section{Status Quo}

Technically the room-to-course or room-to-class ${ }^{29}$ scheduling problem can be interpreted as assignment of a "work piece" to a "server", and vice versa, with certain constraints that have to be respected. With this interpretation it is a matter of definition and of objective, whether the classroom, the teacher, the student body or the course held will be regarded as "server" or as "work piece". Such scheduling and timetabling problems can be approached either via exact algorithms or by heuristics. Regardless of the approach, only constraints that can be formalized may be taken into account by the algorithms. Further, the more constraints a solution has to be obey, the harder it is to come up with one and the longer the algorithm will take to compute one. The size of the respective system adds to the runtime of the algorithm as it increases the search space. Unfortunately the search space, and thus runtime, increase more often than not exponentially with the system's size instead of linearly. This is also the case for "the general course timetabling problem [which] is known to be NP-hard", as stated by MirHassani and Habibi (2011).

While methods for such timetabling-, scheduling- or resource-allocation problems exist, they are found mainly in the industry (especially in production and logistics) or for "plain" timetabling (e.g. organization of night shifts). At universities their application is scarce, if existing at all. One of the main reasons for this is the necessity for incorporation of personal preferences or individual requirements into the scheduling/timetabling task - which is making things really complex. These additional constraints are especially problematic as it is frequently not possible to formalize them. They are thus at least an obstacle to the solution, if not contradictory to its efficiency.

Herein lies a core obstacle of solving the university space management problem via (pure) algorithmic approaches. Even if these produce technically correct results, successful deployment at an institution is unlikely if there are (major) concerns regarding the method or objections against it - even if nonrational. The authors' own experience regarding the incorporation of the human factor into the university space management problem is expressed perfectly by Carter (2001) who wrote: "Practical course timetabling is 10\% [...] theory, and $90 \%$ politics! When we first began designing the system, we were warned: 'You cannot dictate to professors when they will teach courses!' " An opinion shared by McCollum (2007) who describes it as "a balance between keeping all the stakeholders happy". These all are summed up within the analysis of Piechowiak et al. (2005) who put it as:

"Fully automated tools are not efficient when the constraints cannot lead to a valid solution (impossibility of building a clash-free timetable). In these situations, the tools do not provide any support in explaining the causes for the lack of solution. Nothing is given to determine which constraints must be relaxed to bring about a solution. The quality of these timetables also depends on the exhaustiveness of the constraints. In a university, it is impossible to

\footnotetext{
${ }^{29}$ And the other way round: course-to-room and class-to-room, respectively.
} 
collect and to formalize all this information. Expertise of timetablers is the key."

As a consequence Piechowiak et al. $(2005)^{30}$ explicitly distinguish between the two types of constraints, classifying them as "physical constraints (also called hard constraints) and preference constraints (or soft constraints) which are linked to the pedagogical quality of the timetables". The differentiation between these two types is put as follows: "The physical constraints make it possible to be sure that [a] problem has a solution (resolution) whereas the preference constraints are generally taken into account when a solution is being improved (optimization)." For a listing of frequently used objectives, hard and soft constraints in timetabling problems see MirHassani and Habibi (2011).

The problem to transfer theoretical knowledge and solutions into practice was also noted by Carter and Laporte (1998), who were "somewhat surprised to discover that there are very few course timetabling papers that actually report that the (research) methods have been implemented and used in an institution". As this meta-analysis has been published in 1998, things have changed in the meantime. Yet, "many successful university timetabling systems are often applied only in the institutions where they were designed [at]" MirHassani and Habibi (2011). According to them, the reason for this is, "that the [timetabling] system is often tailored to the needs of a particular institution or user." Another, much more drastically formulated, reason is given by McCollum (2007), who states that "researchers, in many cases, have been working on oversimplified problems". In order to overcome these problems MirHassani and Habibi (2011) propose "to provide a method that [is] easily reformulated or customized to support change". This was one of the main considerations when creating the tool that will be presented in section 4.2.1 of this thesis.

\section{Conclusion}

Taking into account the above described difficulties which are surrounding the process of setting up a successful timetabling or scheduling solution, the question is, whether such an approach can satisfy the real world needs of a university-like institution. The importance of the human factor within the system has been repeatedly stressed. Especially the finding of Carter (2001) that "giving timetable reps [representatives; note from the author] the facility to make ... changes was the single most important contribution [to the goodness of solutions]" supports the need for an, at least partially, human-controlled approach.

At Austrian Universities scheduling of courses and events is organized and coordinated "centrally" by the universities' deaneries and/or departments. The hereby produced timetables are a result of many influencing factors, such as historical development and know-how, reaction to newly introduced branches of study, personal preferences of the faculty, etc. For example are groups of courses, especially for lower semesters that seem to be scheduled without causing friction frequently kept that way over years (and decades). Such "introductory" or basic courses usually address a large audience, and while this procedure might not be perfect it works out sufficiently well. Problems arise with changing (i.e. growing) student numbers and the introduction of additional branches of study that have to be woven into the existing schedule. In the course of studies the

\footnotetext{
${ }^{30}$ Amongst others such as, for example, Burke and Petrovic (2002).
} 
initially compact and large student body gets smaller (mainly due to dropouts) and decomposed into smaller units (i.e. partitioning of branches and further specialization). As a result master- and elective courses are most of the time for a much smaller audience and thus can be - and are - arranged more flexibly.

Since faculty seems to be fairly opposed to dictated schedules and on the other hand accustomed to the passed down timetables, it is unlikely that a completely new timetable would be accepted. This naturally hinders an automated timetabling process, as fixing of large parts of the schedule would leave only little room for optimization. Additionally an automated timetable could change drastically from semester to semester (or year to year) because of only small changes in the courses, requiring the faculty to adapt their remaining work (and life) schedule ${ }^{31}$ accordingly. Finally, as already mentioned, high flexibility to meet personal needs is often desired - especially in higher semesters.

Thus in order to increase the space management efficiency this thesis' focus is set on assignment of adequate rooms instead of on the (automated) generation of a timetable. While this approach may seem inferior on paper, it is more likely to be put in place and thus to increase efficiency of real world systems. To solve this task a simulation model, which will be briefly presented in section 4.2.1, is developed. Since this model is not based on an explicit objective function - in its mathematical term - an analytic solution of the problem is not possible. Due to university politics the decision/selection of a space management strategy to be used shall be made by a (human) decision maker and not the model. For this reason the term improvement is favored over optimization in the present case in order to avoid the risk of confusion regarding the mathematical definition of "optimum" and thus the expectation of finding the best solution. Even more so in the case where the final solution is chosen by a human decision maker and thus not mathematically reproducible and/or deducible.

\footnotetext{
${ }^{31}$ Research days, consultation hours, working days if only employed part time, etc.
} 


\section{Chapter 3}

\section{Methodology}

In this chapter the methods used, adapted or developed in order to support the expanded simulation process, as defined in section 2.1.3, will be described. The main focus is hereby put on the extended stages/steps (system analysis and embedding of the model). Starting out with the preconditions (definition of objectives and measurement), methods for system analysis will be covered. These are followed by tools for embedding of the model, with a strong focus on information visualization and the development of the "deployment matrix", which is to assist the deployment processes or to guide through alternatives if deployment is not possible as intended. Finally the mathematical simulation approaches - Discrete Event Simulation, Cellular Automata and Agent-Based methods - will be characterized.

\section{Objectives and Measurement}

As mentioned at the end of chapter 2, the reasons to initiate a simulation project are manifold and usually rest outside of the project itself. Nevertheless they reach into the project with a very important, not to say crucial, aspect choosing and defining the objectives. While this seems trivial and fairly easy, it poses a great threat in practice. The risk is that the reason for initiation of the process will be mistaken as a sufficient explanation or even as definition of an objective. This risk is especially high in cases where the ordering party and the modeling and simulation team are not (very) familiar with the business area of the other.

In some cases the customer does not even know what exactly she or he wants to simulate and what the results should be used for or which decisions they should support. Let's assume that this is not the case in following short example: a project in order to "simulate, evaluate and optimize a new airplane design". If this is "all" the information given, the outcome might be a flightsimulator to train pilots on the new airplane's cockpit and controls, a simulation of the plane's structure under physical stress, a simulation for plane evacuation scenarios, simulation of the behavior and interaction between various on-board systems, etc. While such an incomplete description of the project is unrealistic, it does point out, that an initially - seemingly - "sensible" simulation project is not necessarily a well-defined model objective!

Hence a modeling project should at least question and critically analyze 
the ordering party's assignment - this is if the ordering party is experienced with such projects. To eliminate sources of misunderstanding it is ideal that, at project start, the parties involved work out and define the questions the model should be capable of answering. In extreme cases this can even lead to a completely different (type of) project than what was expected by the ordering party. Although one that covers its actual needs!

While models allow for differing scenarios to be simulated and thus tested, this does not automatically lead to obtaining the desired solution - as explained in section 2.2. The key to this is comparability. However, comparability implies the existence of standardized units of measurement which are applicable for evaluation of all alternatives. Beyond that the units of measurement have to support reaching the objectives and thus comply with them. For completeness it has to be noted, that it is regardless whether measured quantities are contradictory or not, as this has to be dealt with in the course of the weighting process (see section 2.2).

For simulation tasks in common areas identification of adequate units of measurement will most likely be straightforward. Though it can become tricky when breaking new ground and working in uncharted fields - the case in this thesis.

\subsection{System Analysis}

\section{Historical development}

The urge to understand and subsequently to predict the behavior of (complex) "systems" is probably as old as humanity is, as calendars and astronomic observatories, often used for astrological or religious purposes, of ancient cultures (e.g. Stonehenge, Maya, ancient Egypt, etc.) indicate. It was probably Aristotle who first grasped and formulated the nature of the core concept of (complex) systems' in his famous saying "...the totality is not, as it were, a mere heap, but the whole is something besides the parts..." (Aristotle, 350 B.C. (est.), see Book VII, Part 6), which nowadays is best known as "the whole is greater than the sum of its parts" ${ }^{1}$. This quote also serves as link to complex system, as it describes the phenomenon which nowadays is referred to as emergence.

Nevertheless it took natural sciences ${ }^{2}$ several hundred years, until the beginning of the $20^{\text {th }}$ century, to be precise, to pick up on the system-idea. Until then, according to Krallmann et al. (1999), the concept was to be found in philosophy and theology (such as in "Summa Theologica" by Thomas von Aquin or in "Discours de la méthode" by René Descartes), while natural science stuck to the principle of causality ${ }^{3}$ - although with great success!

The turning point, according to Krallmann et al. (1999), was marked by biologist von Bertalanffy who, in the 1920ies, published his findings, that studying single components and processes will not allow comprehensive understanding of the phenomenon of life - a perspective he referred to as "Systemtheorie des

\footnotetext{
${ }^{1}$ While the latter does sound catchier than the original, it contains the error that summing up the parts is not a sensible "operation".

${ }^{2}$ Natural sciences in the modern sense - not to be confused with the ancient understanding of natural philosophy.

${ }^{3}$ It is worth noting that it was Aristotle again who described this principle (apparently) first.
} 
Organismus" (literally: systems theory of the organism). Necessary for the, subsequent, development of the general systems theory was the understanding that generalization of these (biological) system's principles allowed them to be applied to virtually any kind of system. The foundation for general systems theory was laid.

To stick with ancient Greek philosophers, Heraclitus' "war is the father of all things" also holds true for systems theory, which significantly prospered and advanced during World War II. Especially in the US and in Great Britain scientist of various fields, although primarily of technical nature, were thrown together to work and research interdisciplinary, which eventually created new research areas such as operations research and systems analysis.

Computers and the steady increase in computing power where further ingredients. Brought along during the second part of the last century, they allowed systems analysis - and related research areas - to evolve. Computers brought along completely new possibilities and eventually led to the development of system dynamics, probably most famous for Jay Forrester's world model published by the "Club of Rome" in its report "The Limits to Growth". It was this report that triggered discussion about earth's capacity, today better known as ecological footprint.

In its early days systems analysis was mainly applied to mechanic systems and only later to investigate socio-economic or socio-technical systems. A reason for this was the problematic incorporation of the human factor, as, despite assumption, humans do not behave rationally. Thus, as a solution to the problem, human irrational behavior had to be incorporated into systems analysis and development. This happened, to the authority of Krallmann et al. (1999), through the development of participative systems analysis, which strives to include the human behavior of (at least) those directly involved.

\section{Technological, Organizational and Psycho-Social Aspects}

The importance of inclusion of the human factor is especially high when analyzing socio-technical or socio-economic systems, which is explained by the systems' nature. According to Krallmann et al. these are made up of

1. technological,

2. organizational and

3. psycho-social

layers, which are to be analyzed (in an iterative process). As every system is composed of different layer-proportions, analysis and the methods used for it will vary accordingly. It lies within the responsibility of the people in charge to apply adequate methods. Nevertheless a general overview will be the starting point for the vast majority of processes.

Identification of the system, its relevant environment and the elements within both provides such an overview. This is also the standard approach described in respective literature, such as Ulrich and Probst (1991); Krallmann et al. (1999); Ulrich (2001); Schalcher (2008). It classifies elements according to their "position" - inside or outside of the system. As visualized in Fig. 3.1 elements located outside of the system's boundaries are further classified as within the 
relevant environment or outside of it. Those belonging to the latter class are of no relevance to the system.

This procedure transcends the core system's boundary, the outside environment too is becoming subject of the investigation. While this may be irritating or even scary from a technocratic perspective, it is necessary if the analysis should not be an end to itself. Or, as Ulrich and Probst (1991) put it ${ }^{4}$ : "technical systems are only of value if they are incorporated into social systems." And it is this that justifies the expansion of the simulation process (as described in sec. 2.1.3).

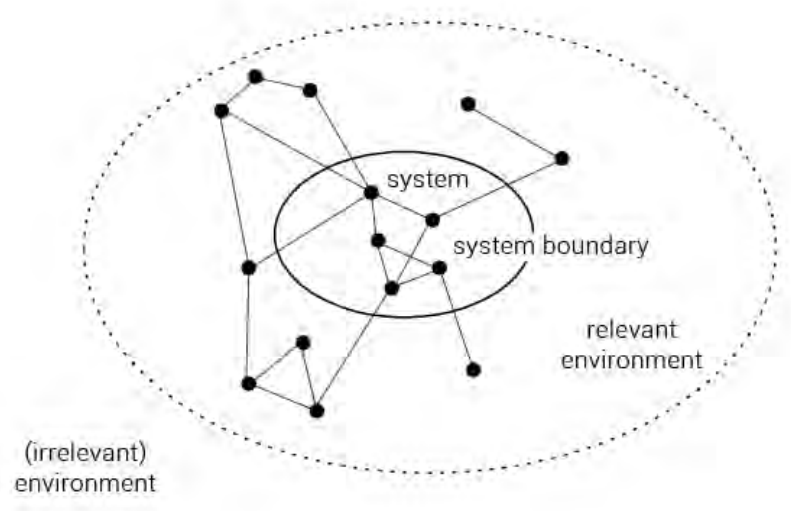

Figure 3.1: Sketch of a system with in- and external elements, relations between elements, the system boundary and a relevant sphere of the environment (source: Schalcher (2008, p. 2-4)).

Although it might not be necessary to formally carry out this preliminary overview (e.g. for small systems and people routinely involved in "systemthinking"), the insight gained is very helpful for the subsequent analysis of the three layers. It provides a first holistic perspective and allows for identification of (vital) system input (i.e. elements in the relevant sphere of influence) and possibly for assessment of the systems' influence upon its environment. With input elements being of special importance as they (usually) are beyond control from within the system but with a big effect on its behavior.

As mentioned before, system analysis is an iterative process that switches between the three layers. It is obvious that the layers' differing nature calls for specific analysis approaches for each. While analysis of the technological layer has to deal with clearly formulated processes and structures (e.g. physical or electromechanical laws), a precise description or definition of processes at the organizational layer is harder to obtain. And, finally, at the psycho-social level it's the fuzzy extreme of well-defined laws of nature. Consequently insights from every level support different aspects of the modeling process.

Roughly speaking analysis of the technical layer can be regarded as closely related to step 2 of the simulation process ("modeling" as defined in 2.1.3) - its findings are main contributions to effective model implementation. Of course insights gained from analysis of the systems' remaining two layers - the orga-

\footnotetext{
${ }^{4}$ German original “. . . technische Systeme machen nur Sinn, wenn sie als Elemente in soziale Systeme eingegliedert sind.", translation by the author.
} 
nizational and psycho-social - also influence the actual implementation. Nevertheless information extracted from organizational analysis will more often be used to support the core model and its deployment. This also includes assessment of data and data-sources, of business processes and potential modifications or of suitable specification for representation of simulation results. This in turn influences the design of the model (e.g. design of data-interfaces or the behavior of entities) or immerses into psycho-social aspects respectively (e.g. preattentive processing of information visualization). Information gathered during psycho-social analysis can be essential for both, the implementation of the core model (e.g. integration of irrational user-behavior) as well as the integration of the model within the system itself (e.g. formally nonexistent business processes need to be considered and implemented). In short: the borders between the layers are blurry, which is a major cause for the iterative nature of the analysis process.

A widely used method to analyze the technical layer are causal loop diagrams, as known from system dynamics (see example in Fig. 3.2). This representation facilitates understanding of elements' mutual influences and identification of feedback-loops. It also allows classification of a system's elements, via the socalled "influence matrix", which is depicted in Fig. 3.3 (as described by Ulrich and Probst (1991)). Every element's influence on all other elements is rated and stored row-wise in the matrix (e.g. $0=$ no influence, $1=$ weak influence, $2=$ substantial influence and $3=$ severe influence). Ratings are then summed up row- and column-wise, which gives totals for each element's influence and controllability. Now it is possible to classify them as either "reactive", "critical", "inert" or "active", depending on their influence-controllability relation (see Table 3.1).

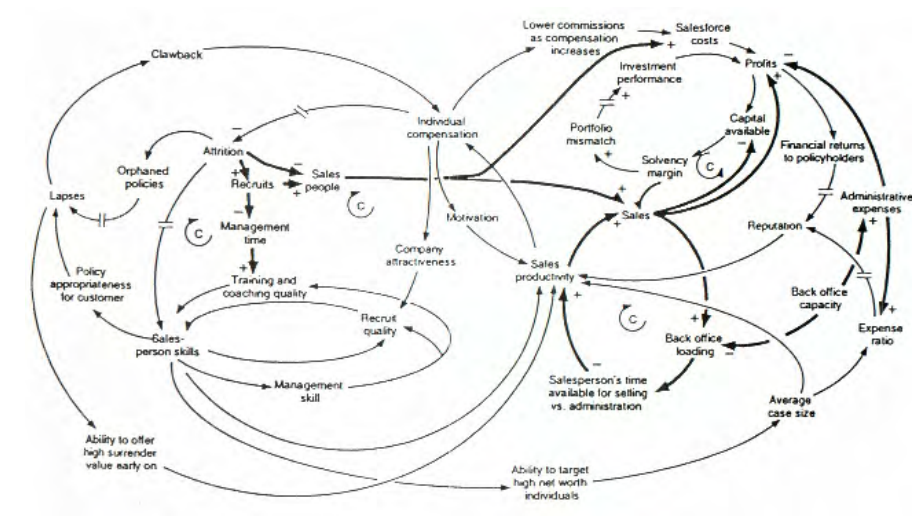

Figure 3.2: Example of a causal loop diagram used in a system dynamics model (author: Robert A. Taylor, U.S. Department of Energy; image obtained via WIKIPEDIA ${ }^{5}$.

Elements with a high influence (i.e. active and critical) can potentially be used as controllers for the system. Since critical elements are themselves easily influenced, manipulation can trigger (potentially fatal) oscillations in the feedback-loops, leaving only active elements as a sensible option for controllers. Reactive elements can be monitored and used as indicators or early warning

\footnotetext{
${ }^{5}$ Website: http://en.wikipedia.org/wiki/File:Causal_Loop_Diagram_of_a_Model.gif last checked: September 16, 2013.
} 


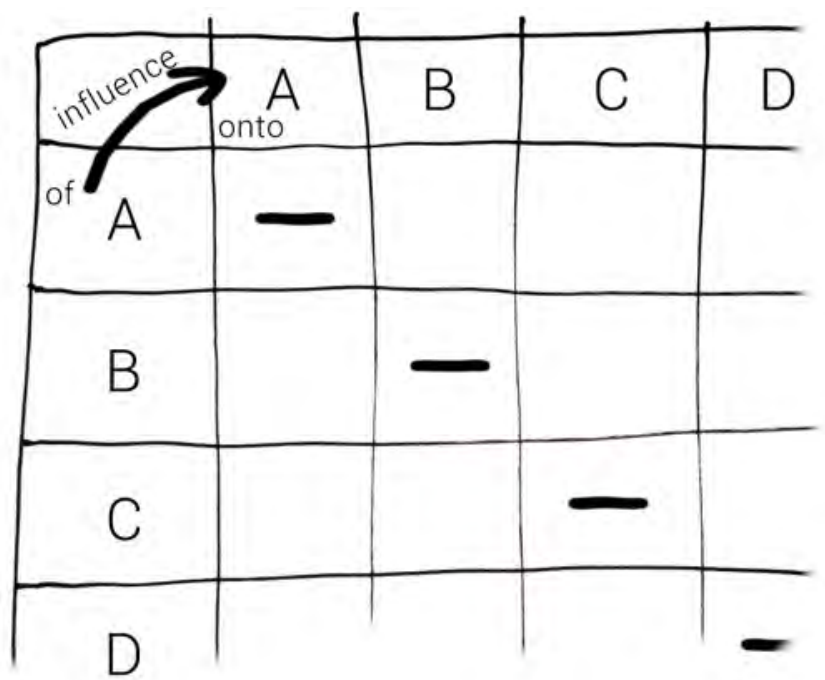

Figure 3.3: Sketch of an influence matrix.

Table 3.1: Classification of elements by influence-controllability relation

\begin{tabular}{c|cc} 
& low influence & high influence \\
\hline low controllability & "reactive" & "critical" \\
high controllability & "inert" & "active"
\end{tabular}

signals since they will react easily (and thus relatively) early on changes within the system.

Causal loop diagrams, and system dynamics, are classified as top-down approaches. Top-down approaches describe the system "as a whole" from a topperspective. This works fairly well when modeling systems whose entities can be aggregated and thus mapped onto stocks and flows (e.g. amount of goods in stock, money, total number of employees, etc.). But they fail (or become overly complex and complicated to set up) if a system's elements cannot be summed up or when it becomes necessary to distinguish the instances of an element (e.g. modeling of the employees as individuals instead of the employee headcount). For such cases bottom-up approaches, such as agent-based methods or cellular automata, are better suited. As the latter is the case of the present problem to increase the utilization-efficiency of lecture space, a combination of bottom-up methods has been harnessed. In this thesis the methods used will be introduced shortly and a brief overview of the hybrid model will be given. Reference is made to Tauböck (2010) for a thorough technical description of the model.

Due to the nature of the psycho-social layer's analysis, methods from social sciences and psychology need to be applied. They are not covered within this thesis as their exploration would go far beyond its technical focus. In addition, as will be explained later, the team conducting the case study project was part of the investigated system (Vienna University of Technology) and thus relied on gut instinct and informal knowledge for analysis of the psycho-social layer. 
The third - organizational - layer also acts as putty between the precise technological and the fuzzy psycho-social layer. Via analysis of business processes it is possible to incorporate the human factor (partially, i.e. usually without irrational behavior). At the same time business processes are fairly precise and explicit, thus able to be translated into a technical model description. Methods used to support the analysis process of the organizational layer are numerous. With some of the famous being Structured System Analysis (SSA), Structured systems analysis and design method (SSADM), System or Structured Analysis and Design Technique (SADT), Data Flow Diagrams (DFD), Entity Relationship Modeling (EMD), Unified Modeling Language (UML) and Business Process Modeling (BPM) with its special Business Process Modeling Notation (BPMN). Deciding which method is best suited will depend on the given case and thus on the people involved in the respective project.

In the present case the Business Process Modeling Notation (BPMN) has been chosen and adapted in order to better suit the projects needs. For purposes of a complete system analysis it was accompanied by a Stakeholder Analysis and Entity Relationship Modeling (ERM) to develop an Entity Relationship Diagram (ERD).

\subsubsection{Business Process Modeling Notation}

The Business Process Modeling Notation (BPMN) provides a way to produce Business Process Diagrams (BPD), with a primary focuses on the graphical presentation (notation). It has been developed by Stephen White and released to the public in its version 1.0 via the Business Process Management Initiative (BPMI) in May 2004 ${ }^{6}$. According to White (2004) the notation produces BPDs which are "based on a flowcharting technique tailored for creating graphical models of business process operations. [The resulting] Business Process Model, then, is a network of graphical objects, which are activities (i.e., work) and the flow controls that define their order of performance." In the official BPMN specification (see (Object Management Group, 2008)), the main goal behind the development of the notation is defined as, providing "a notation that is readily understandable by all business users, from the business analysts that create the initial drafts of the processes, to the technical developers responsible for implementing the technology that will perform those processes, and finally, to the business people who will manage and monitor those processes. Thus, BPMN creates a standardized bridge for the gap between the business process design and process implementation."

\section{Classical BPMN}

As stated above, a BPD is made up of graphical objects for which the BPMN strives to set a standard graphical notation. In order to make it easier for readers and/or modelers, the number of distinct shapes is limited to the subclasses of elements; elements within these sub-classes use variations of the main presentation. While the primary focus of the BPMN is clearly set on the notation, it also defines the semantic (meaning) of the symbols, but it attaches less value to this aspect. A reason for this might be to allow the notation to be as

\footnotetext{
${ }^{6}$ In the meantime specification version 2.0 of the BPMN is available, see http://www.omg.org/spec/BPMN/.
} 
flexible as possible to thus ensure compatibility with as many engineering- and IT-languages as possible.

BPMN classifies elements regarding their general purpose into four basic categories which are further split up. These categories and sub-categories are $^{7}$ :

- Flow objects (see Fig. 3.4a)

- Events ... something is "happening" during the (business) process. Events usually have a cause (trigger) or an impact (result). There are three types of Events, based on when they affect the flow: Start, Intermediate, and End. Their graphical representations are circles with open centers that are reserved for internal markers, which differentiate different triggers/results (see Fig. 3.6 for a listing).

- Activities

...represent "work" that is performed. This can either be single tasks or (sub-)processes. Graphically activities are represented by rounded-corner rectangles ${ }^{8}$. Collapsed sub-process, which are indicated by a "+" sign in the lower-center of the rectangle, contain closed BPMN-processes within.

- Gateways

... are represented by a diamond shape and diverge or converge the sequence flow of the process. As with events, internal markers indicate the type of behavior (see Fig. 3.6 for a listing).

- Connecting objects (see Fig. 3.4b)

$\ldots$ are all represented by arrows.

- Sequence flows

... show the order in which activities are performed form the start event to the end event. The arrow is a solid line with a solid head (note: there are several sub-types of sequence flows).

\section{- Message flows}

... depict the exchange of messages between different participants of the process. The participants are represented by different pools (see swim lanes) in the BPD. The message flow's notation is a dashed line with a small empty circle at its beginning and an empty arrowhead at its end.

- Associations ... are used to associate data, text and other artifacts with flow objects. They are represented by dotted lines with a line arrowhead.

- Swim lanes (see Fig. 3.5a)

... serves as graphical containers for activities of participants.

\footnotetext{
${ }^{7}$ See White (2004) for a brief overview and Object Management Group (2008) for an extensive description. The following descriptions are extracted from, or based on these sources.

${ }^{8} \mathrm{~A}$ design for which Apple was granted a patent by the US Patent and Trademark Office as of November 6, 2012 (Patent-No.: D670,286). Imho: crazy.
} 


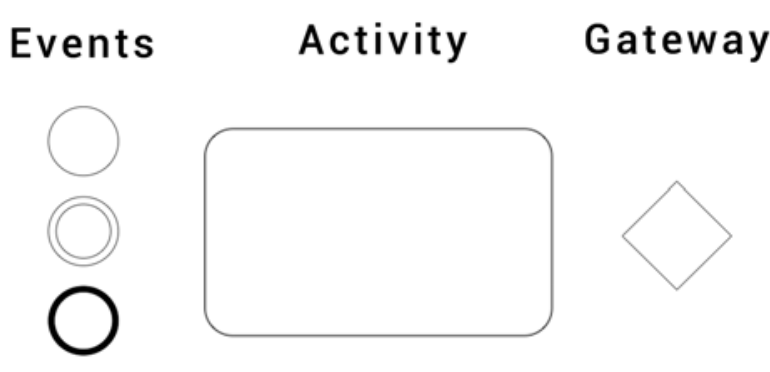

(a) BPMN Flow Objects (from left to right): Events (from top: Start, Intermediate, End), Activity, Gateway (source: White (2004)).

\section{Sequence Message Association}

(b) BPMN Connecting Objects (from left to right): Sequence flow, Message flow, Association (source: White (2004)).

Figure 3.4: Basic elements of the Business Process Modeling Notation (BPMN) classes Flow Objects and Connecting objects.

- Pools ... represent one specific process participants (i.e. one pool per participant); can be aligned vertically or horizontally.

- Lanes

... are sub-partitions of pools, they extend the entire length of the pool and are used to organize and categorize activities within pools.

- Artifacts (see Fig. 3.5b)

... do not have a direct effect on the sequence or message flow. They are used to extend the basic (BPMN) notation and to add context and thus give modelers a certain extent of flexibility.

- Data Objects

...provide information on what activities require to be performed and/or what they produce. They are connected with activities via associations.

- Groups ... are represented by a dashed rectangle with rounded corners. They are used for documentation or analysis purposes.

\section{- Annotations}

... also called text annotations, are used to provide the reader of the BPD with additional information.

With these elements and the BPMN specifications (even more with those of version 2.0) it is possible to create very detailed presentations of business processes which can be also be used as technical implementation guideline. 
Pool ...

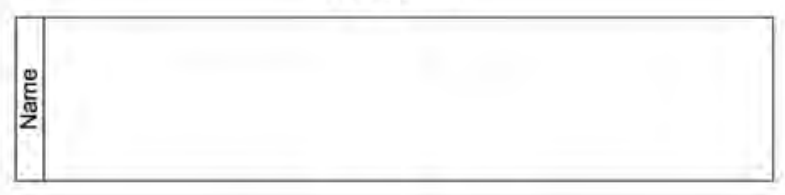

with 2 lanes

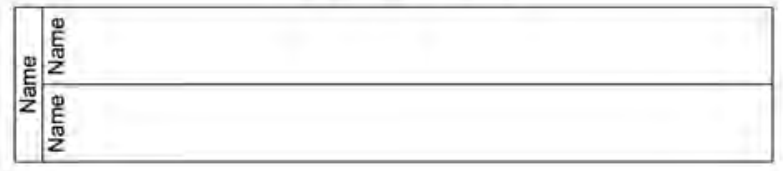

(a) BPMN Swim Lanes: Pool (top), Pool with two Lanes (bottom; source: White (2004)).

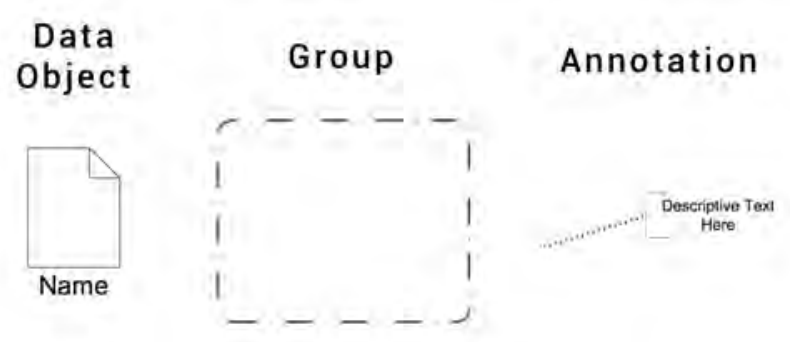

(b) BPMN Artifacts (from left to right): Data object, Group, Annotation (source: Object Management Group (2008)).

Figure 3.5: Basic elements of the Business Process Modeling Notation (BPMN) classes Swim Lanes and Artifacts.

Figure 3.7 shows a BPMN (version 1.1) example: an email-voting process ${ }^{9}$ which contains two collapsed sub-processes ("Discussion Cycle" and "Collect Votes" - see Fig. 3.8 for expanded processes) and one expanded (unnamed, in the upper right corner, containing only two actions). Besides these three subprocesses there are 21 flow objects ( 5 events, 10 activities and 6 gateways) in the process model - thus a total of 24 elements, neglecting connecting objects.

When also counting the flow objects and artifacts of the sub-processes one ends up with 65 elements in this fairly simple example. The numbers of elements of each (sub-) process are displayed in Table 3.2 - neglecting only connecting objects, as the model does not contain any swim lanes.

The author is aware of the fact that four actions, two events and one gateway are counted twice, as they are also displayed with the sub-process "Collect Votes" in Fig. 3.8b. The reason for the double counting is, that these elements are depicted twice as they are deemed necessary for convenient reading of the model. Subsequently they add to the visual noise of the diagram. Besides, if the number of elements were to be reduced by expanding the sub-processes within the top-level of the model (i.e. the two collapsed sub-process elements

${ }^{9}$ Taken from the official specification of version 1.1 (see Object Management Group (2008)). 
Triggers

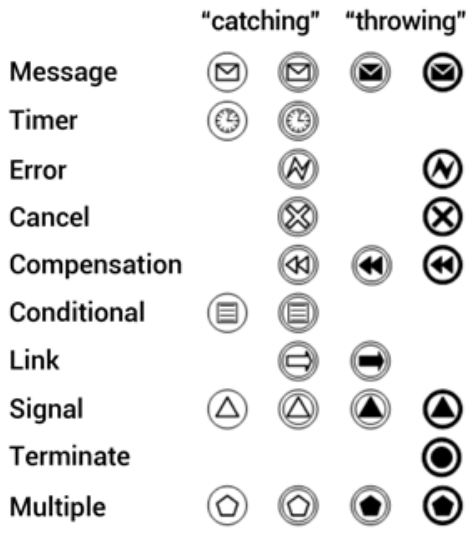

Gateways

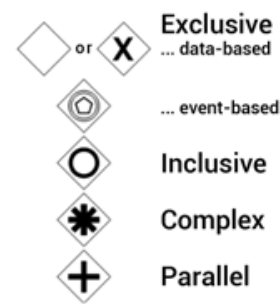

Figure 3.6: Incomplete selection of Trigger- and Gateway-symbols defined in the BPMN specification (source: Object Management Group (2008)).

Table 3.2: Elements of top-level process and sub-processes of the example process depicted in Fig. 3.7 and 3.8.

\begin{tabular}{ccccccc|c} 
& Events & Actions & Gatew. & Data Obj. & Annot. & Subs & Sum \\
\hline Top & 5 & 10 & 6 & - & - & 3 & 24 \\
Sub 1 & 5 & 6 & 4 & 2 & 3 & - & 20 \\
Sub 2 & 6 & 10 & 2 & 3 & - & - & 21 \\
\hline Total & 16 & 26 & 12 & 5 & 3 & 3 & 65
\end{tabular}

are replaced by the elements contained within the actual sub-processes) it would save nine elements (2 sub-processes, plus the previously mentioned 7 duplicate elements) but the readability of the model would arguably suffer — despite the lower overall number of elements.

Nevertheless, visualizing all of these elements in one diagram, i.e. without collapsed sub-processes, is still possible at the complexity-level of this voting process. But, as argued before, the diagram is becoming visually crowded. It is obvious that more complicated and/or larger processes require splitting and grouping in order to embrace all details. In both cases (visually crowded or fragmented diagrams) this is making it hard for people not familiar with the notation to read and understand them - which in the first place has been one of the reasons for the development of the BPMN.

Naturally complex problems and processes require adequate solutions and it will most likely not be possible to conceptualize them without complex business process diagrams. This hold especially true if such a diagram is to serve as development blueprint for IT-implementation. Hence, large and complex projects will require training those involved in reading and understanding of BPD. Its scale will thereby depend on the specification of the projects as well as on economic, financial and temporal limitations. 


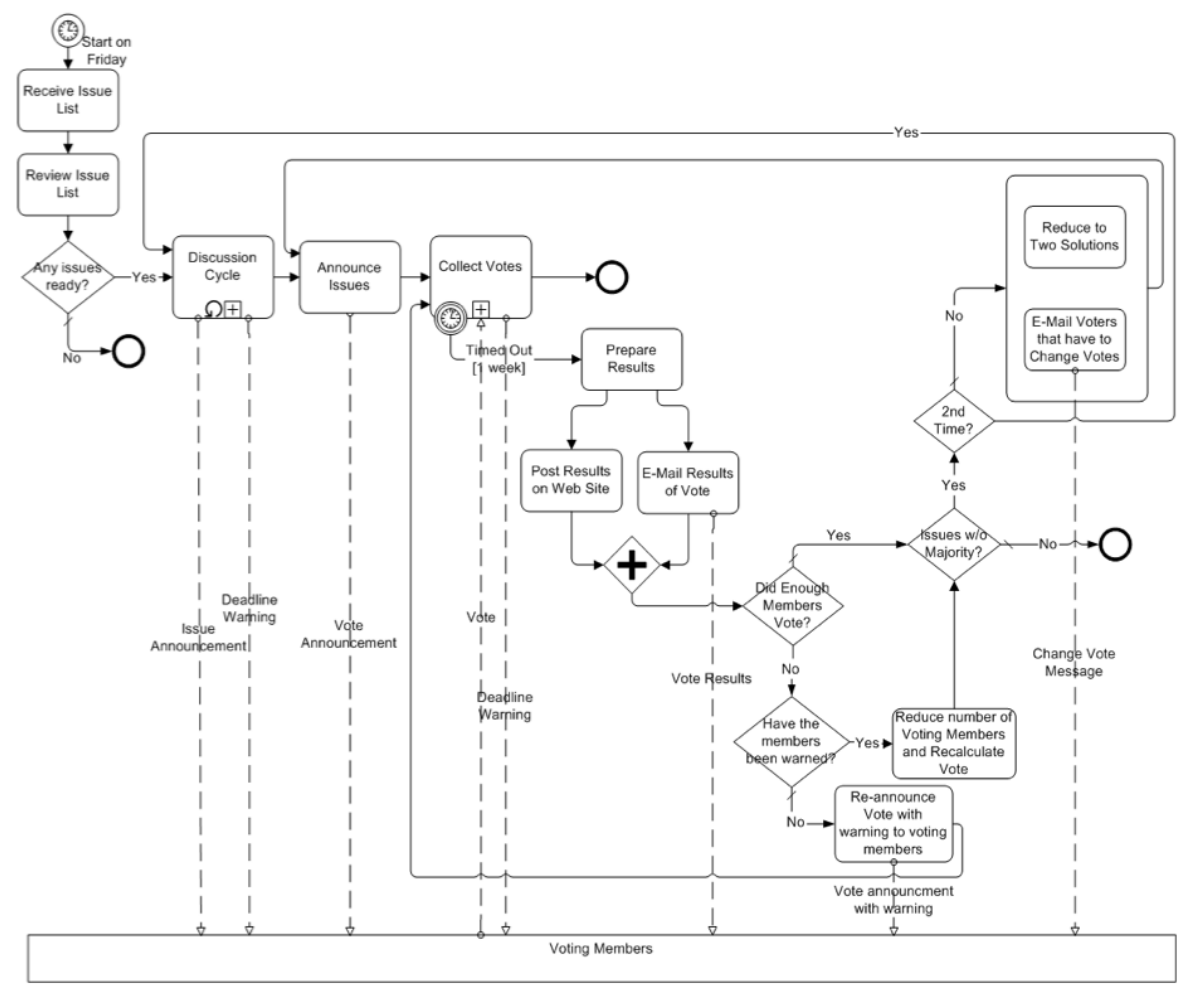

Figure 3.7: Email-voting process as an example for a BPMN diagram; with collapsed sub-processes "Discussion Cycle" (expanded in Fig. 3.8a) and "Collect Votes" (expanded in Fig. 3.8b; source: Object Management Group (2008)).

\section{Simplified BPMN}

Initially the Business Process Modeling Notation has been used for the case study project at Vienna University of Technology. But during the course of the project it was found that it has some shortcomings: even though the notation is fairly intuitive, its visual overload was hindering communication with the involved stakeholders. The reason for this was that the project was of limited size (and budget) and hence integration of the stakeholders into system analysis was only possible to a very limited extent. Subsequently the stake holders were not in contact with BPMN diagrams on a regular basis. Training them on reading and understanding the notation would have been uneconomic ${ }^{10}$. With the BPMN's regulations and specifications being beyond the level necessary for this project, an alternative way to communicate the business processes, correctly but in a simplified version, had to be found.

In this respect the small size of the project proved to be beneficial. As the team conducting the case study was small as well ${ }^{11}$, team-internal communica-

\footnotetext{
${ }^{10}$ For the sake of completeness: such training would most likely have been also problematic from a psychological aspect, as the project was not welcomed by everybody.

${ }^{11}$ While a total of 20 people (not counting stakeholders outside of the project team) have been directly involved in the project over its four year span, the core team fluctuated between three and six people - depending on the active working packages and work load.
} 


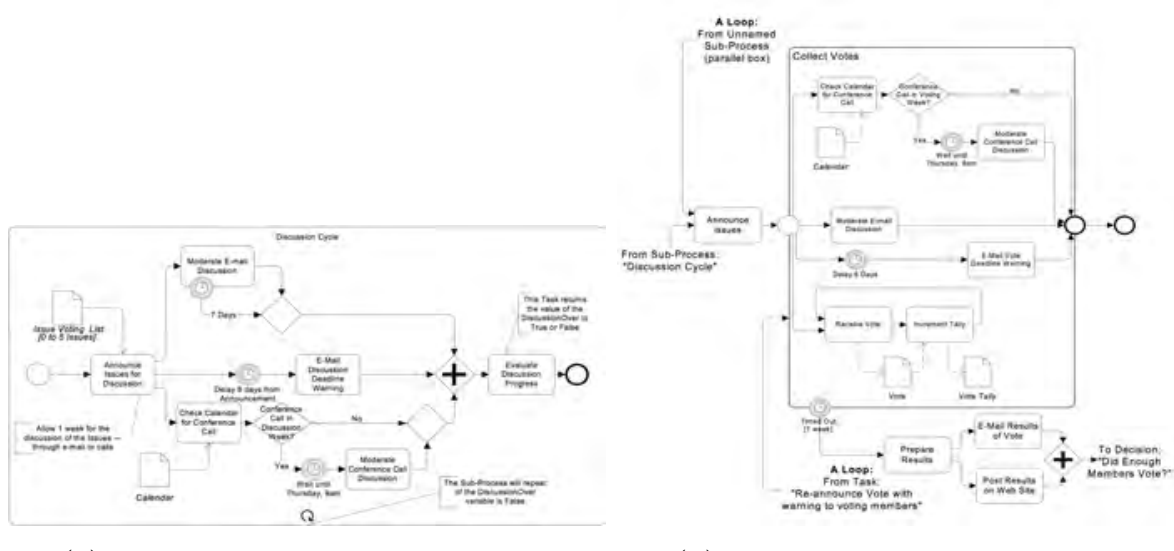

(a) Sub-process "Discussion Cycle"

(b) Sub-process "Collect Votes"

Figure 3.8: Expanded sub-processes "Discussion Cycle" (left) and "Collect Votes" (right) from email-voting process in Fig. 3.7 (source: Object Management Group (2008)).

tion was very direct. Because of this there was no need for a BPD that serves as a technical implementation guideline but merely as a general overview of the business processes and a mean of communicating them to the stakeholders involved. For this a simplified notation was derived from the original BPMN adapted for the requirements of the case study project at hand.

This understanding is supported by Mendling et al. (2007) who empirically investigated "understandability as a proxy for quality of process models and focus on its relations with personal and model characteristics", for which they conducted a questionnaire-based experiment in classes at three European universities. Validation of the experiments' results and findings was done by interviews with practitioners.

Mendeling et al. point out that "there is only little empirical work reported on process model quality and its impact factors" and can only provide their own (empirical) work as references in this direction. From this work they do derive one of their hypotheses: "that human modelers lose track of the interrelations of large and complex models due to their limited cognitive capabilities" — which they see backed up by "Science of the Artificial" from Herbert A. Simon. They also see that the results of these previous investigations "reveal that an increase in size of a model appears to have a negative impact on quality." Interestingly they find that "small variations between models can lead to significant differences in their comprehensibility" even though that the questionnaire's findings, validated by practitioners, "seem to underline the insight that model size is of dominant importance on model understandability".

The combination of these findings, the experiences made by the case study project team and the urge to use a tool as simple as possible - but not any simpler -, lead to the adaption of the BPMN (version 1.1). The resulting, stripped down notation still relies on the four basic BPMN classifications (flow objects, connecting objects, swim lanes and artifacts) and on all of its elements. It does, however, reinterpret the meaning of the message flow as data flow. With a special flow-item for the handover of data the necessity to explicitly use data 
objects within the models became obsolete. The main change from the BPMN is definitely the strongly reduced variety of trigger- and gateway-symbols.

Only the trigger symbols link and message (see Fig. 3.6) were used in the notation for the case study project. The remaining ones were not explicitly excluded and could thus be used if required. Regarding gateways it was possible to limit their use to exclusive "yes or no" and binary decisions.

These limitations were partially possible because of the observed system's nature. Furthermore the simplification itself was only possible as a consequence of this "simplified BPMN" use within the case study project. Within the project team the primary use of the BPMs developed was to identify involved stakeholders, their interconnection, flaws and/obstacles in processes and to extract information regarding (input) data. Outside of the project team the BPMs were used to verify and validate them in cooperation with the stakeholder and to communicate necessary process modifications (as well as preconditions). Thus the process models only needed to roughly identify the business processes. This allowed for a simplified representation, as the BPM did not need to embrace all process details.
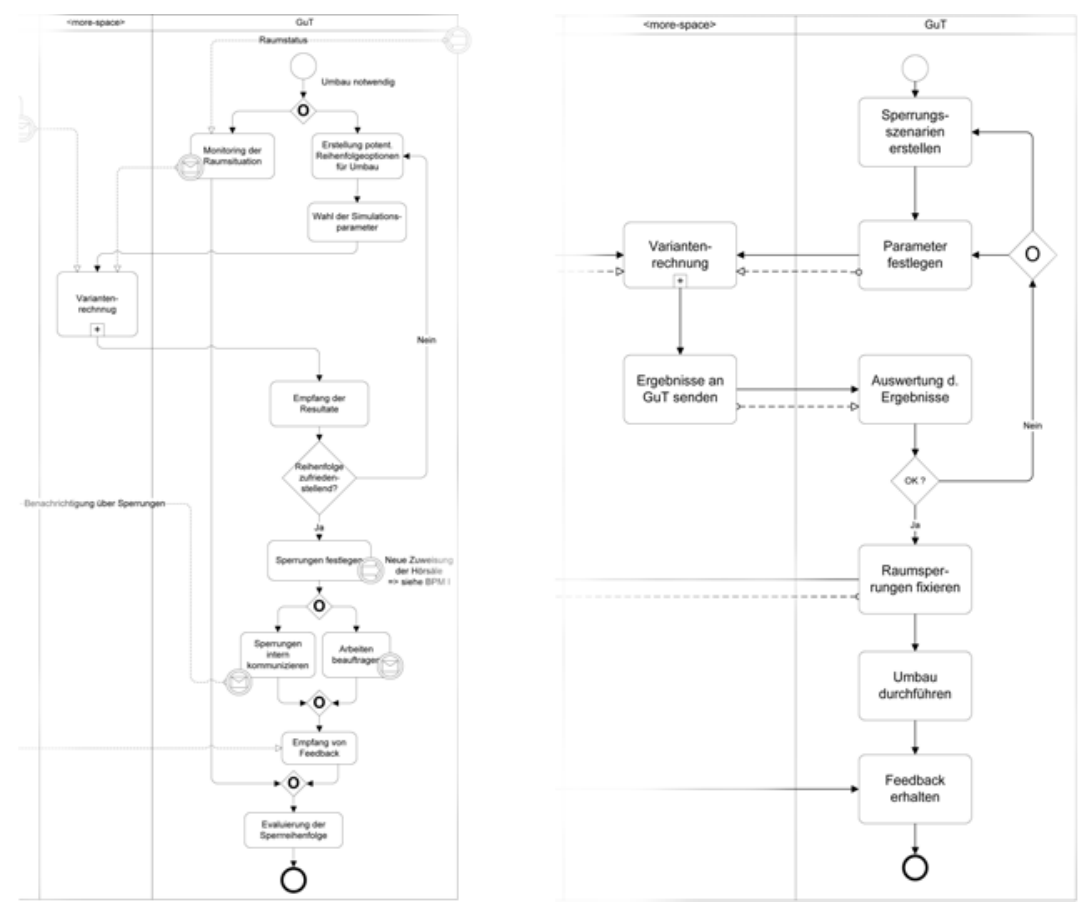

Figure 3.9: Business process fragment in original BPMN (version 1.1, left) and simplified notation (used in the case study project, right).

The effect of the modifications is visible in Fig. 3.9 which shows a business process fragment in both, the original BPMN (version 1.1) and the simplified notation used for the case study project. It is evident that the simplified notation strongly reduces visual noise and improves readability, especially for untrained readers - though at the cost of neglecting detail. 


\subsubsection{Complementary Methods Used}

Besides business process modeling two additional methods were used during system analysis: "Stakeholder Analysis" (SHA) and "Entity Relationship (ER) Modeling", which produces ER diagrams. These techniques complemented the BPM-mapping of the project environment. The stakeholder analysis was used to support the development of the process model, while the ERD helped to assess the quality and structure of (input) data.

\section{Stakeholder Analysis}

Stakeholder analysis is a relatively new tool originating from change projects and policy making. Over the recent years it is slowly finding its way into general project management. The idea behind SHA is to identify all stakeholders involved along with their concerns and interest in order to effectively and actively manage conflicting interests. As a projects fate can be doomed by "unfavorable behavior" of crucial stakeholders, SHA should be carried out at the very beginning of a project. It is desirable to do this even before announcing a project in order to inform the stakeholders and include them in the planning process as good as possible. Hereby it is possible to avoid misunderstandings, circulation of (bad) rumors and active opposition at much lower costs than at a later project stage. Ideally SHA and management will help to generate project outcomes that are accepted by the vast majority through inclusion of all stakeholders.

Formulation of the (project) goals marks the starting point of any SHA. It is followed by the identification of all stakeholders - regardless of whether they are involved or affected directly or indirectly. The next step is to choose the stakeholder characteristics to be analyzed, which has to be in accordance with the project goals set. Thereafter one can start with the actual analysis of those characteristics. This is usually the most time/resource consuming part of the analysis process, as it will most likely require in-depth research and interviews with the stakeholders. During the research an ear should be given to (potential) alliances between stakeholders, which can influence the classification that is to come next.

Based on the information from the research stakeholders will be classified. There are several methods that can be applied in this stage; a very straight forward one, leading to a good overview, is to distinguish the power as well as the interest of every stakeholder and place her in a four-quadrant graph (see Fig. 3.10 for the graph) according to this estimate. It is very helpful to further break down classification by adding stakeholders' positions. One option to add this valuable info to the presentation is to use traffic light color coding for the stakeholder symbols (e.g. green marks for supporters, orange for neutral ones and red for opposed stakeholders), as shown in Fig. 3.11.

This graphic classification facilitates clear stakeholder identification and hereby supports the project management as it now can take (special) care of the most crucial stakeholders - key-stakeholders, with high power and high interest. Nevertheless those with less interest but high power can, if becoming discontent, turn into ones with high interest, turning them into opposing key stakeholders! Similarly stakeholders with a high interest but low power could form alliances and gain power. It is the responsibility of stakeholder management to actively approach the (individual) stakeholders according to their classification, position 


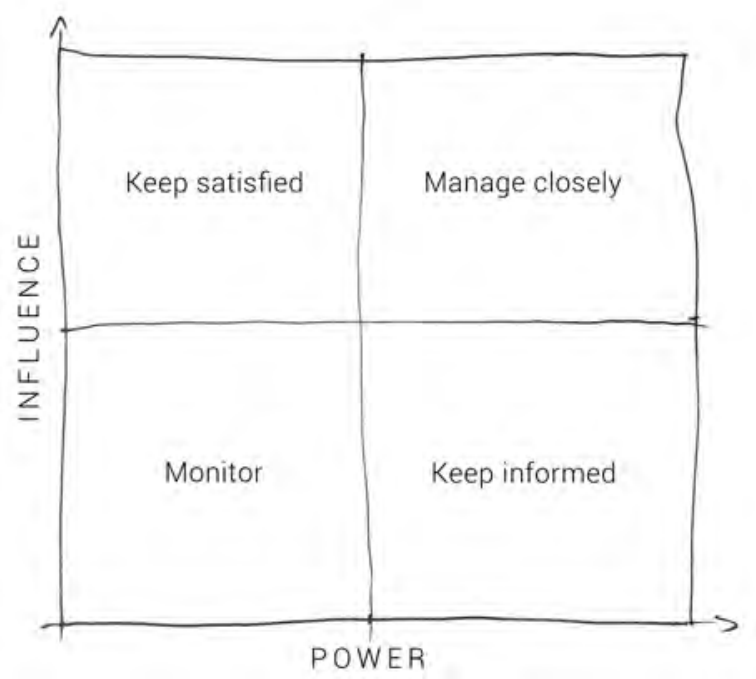

Figure 3.10: Four sector graph for classification of stakeholders according to power and interest.

- and the resources available. Concluding the SHA process is roughly structured as follows:

1. Definition of project goals and planning of the process

2. Identification of all stakeholders

3. Selection of relevant criteria

4. Research/collection of information

5. Stakeholder classification

Hereby generated information should then be used for active stakeholder management in order to reach the project goals.

In the case study project SHA has been used as a complement to the BPM inasmuch, as the key-stakeholders identified (high power, high interest) are very likely to be participants within the business process. Thus the SHA, next to its original purpose of keeping the stakeholders content, presented a mean of validation. This is especially helpful when the BPM is not depicting an existing process but defined a completely new process. In this case the SHA's original purpose, namely inclusion, classification and management of stakeholders, is a very important one.

\section{Entity Relationship Modeling}

The early 1970ies saw the advent of "very large" databases and subsequently data models, such as IBM's IMS database system (hierarchical model) or Honeywell's IDS database system (network model) emerged, according to Chen (2002). As he explains, the relation model was introduced with "considerable interest 


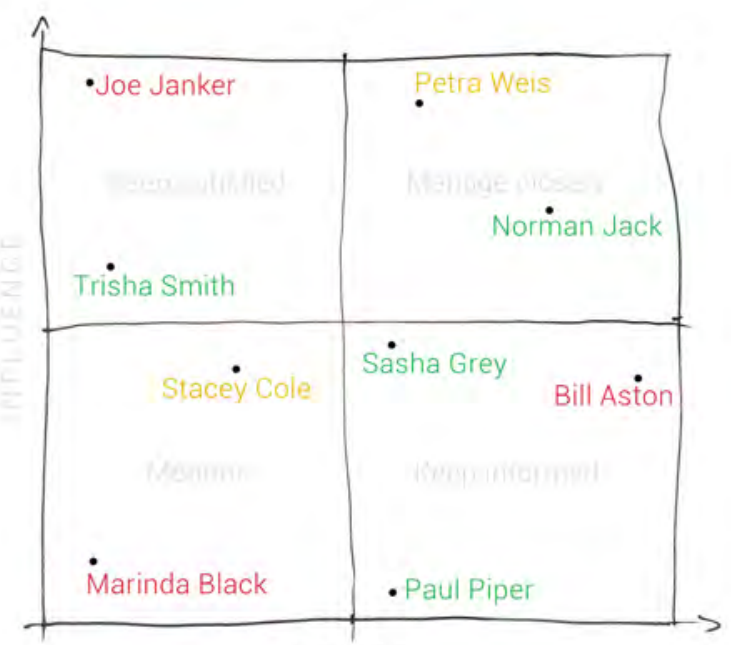

Figure 3.11: Classification of stakeholders with color-coded attitude towards project goals.

in the academic world" in 1970. Later, in 1975, Chen himself presented the first paper describing an ER model, which was published a year later.

In this paper, (see Chen, 1976), he describes the superiority of the ER model over the, then competing, three approaches (network models, relational models and entity set models). It is the combination of "most of [their] advantages" within one single approach while it "adopts the more natural view that the real world consists of entities and relationships". Chen advocates this on grounds of "four levels of views of data" which are ${ }^{12}$ :

1. Information concerning entities and relationships which exist in our minds.

2. Information structure-organization of information in which entities and relationships are represented by data.

3. Access-path-independent data structure-the data structures which are not involved with search schemes, indexing schemes, etc.

4. Access-path-dependent data structure.

Of these the entity set model describes levels 1 and 2 , the relational 2 and 3 , and the network model primarily level 4, while the ER model sums up all of them.

Chen also proposes a diagrammatic technique for such ER models - the entity-relationship diagram (ERD) -, a design process for the respective databases, as well as operations and rules for information retrieval, insertion, deletion and updating of entries. Despite of several different ERD-notations being around nowadays, Chen's basic definition is still valid. The underlying idea of ERM is to (1) identify all (relevant) sets of entities as well as the sets of relationships, (2) analyze the relationship-sets' semantic information, which is followed

\footnotetext{
${ }^{12}$ Level definition cited from Chen (1976).
} 
by (3) a definition of the respective attributes or value-sets, finally (4) the data is organized as entity-relationship relations with specific "primary keys" (i.e. keys respectively labels that uniquely define each relationship/record).

The graphical representation of ERDs varies depending on the chosen notation. Chen's original proposal (see Fig. 3.12a) models the entities as rectangular boxes and relation-descriptions as diamond-shape boxes. If two entities are in a relationship then a line, with a diamond shaped description of the relationship, is connecting them. In addition the mapping of the relationship is specified by figures next to the entities outgoing lines. In such a manner a $1: 1,1: n^{13}$ or $n: m$ mapping can be indicated. In the latter case both $m$ and $n$ indicate "many" and may be, but don't have to be equal $(m=n$ or $m \neq n)$. The notation does not distinguish between these cases, which explains its convention to write "N : $\mathrm{N}$ " for all possible "multiple"-relationships (i.e. $n: n, n: m$ and $m: n)$.

In Fig. 3.12a the relationship's mapping is $1: n$, indicating that every person has (exactly) one location as its birthplace, while one location can be birthplace to many persons.

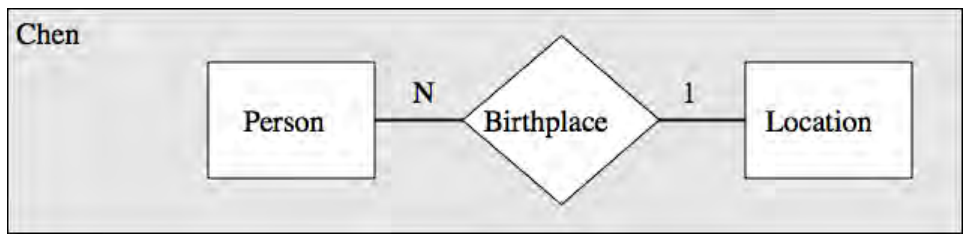

(a) Chen's ERD notation

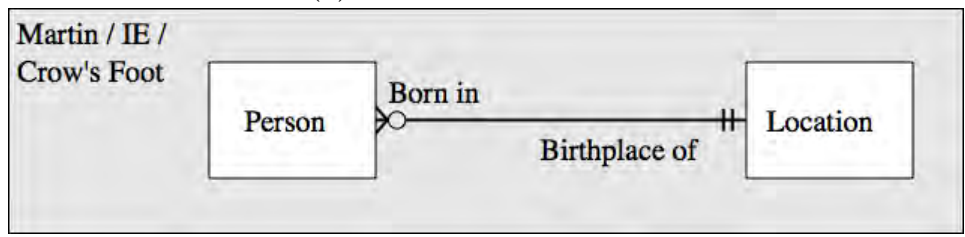

(b) ERD notation "Crow's foot"

Figure 3.12: ERD notations (Chen's at top and "Crow's foot" at bottom) representing an entity-relationship where one location (entity) may be the birthplace (relationship) of multiple persons (entity) and every person has exactly one birthplace (author: Benthompson, image obtained via WIKIPEDIA $\left.{ }^{14}\right)$.

As previously mentioned the precise graphical definitions vary depending on the used notation. Within the present research project both notations, or more precisely: variations of both notations (Chen's and the "crow's foot") have been used. Within the used Chen-based version the convention to denote a $1: n$ relationship as " $1-(1, n)$ ", which brings it a bit closer to the crow's foot notation which specifies the complete mapping interval of the entities.

Of the crow's foot notation several, slightly varying, versions exist. It is further known under the names "Barker's notation" or "information engineering (IE)" notation. Its appeal lies in improved readability and efficient use of

\footnotetext{
${ }^{13}$ And vice versa, i.e. $n: 1$.

${ }^{14}$ Website: http://en.wikipedia.org/wiki/File:ERD_Representation.svg last checked: September 16, 2013.
} 
drawing space, as it omits the diamond-shaped box. Instead relations are, if at all, written directly above the connecting line, while the mapping is indicated by pairwise combinations of three markers: $\bigcirc$ for "none", | for "one" and the crow's foot $\rightarrow$ for "multiple". These pairs declare the possible mapping intervals. Thus $>\bigcirc$ in Fig. 3.12b implies that one location might have zero to multiple persons being born in it. From the other direction the mapping interval II declares that a person must have exactly one birthplace (location). The used notation further enhances the information content of the produced diagram as it adds attributes and their value-sets into every entity's (rectangular) box. Using such ERM techniques it is possible to describe and model large sets of relational data with the entities' respective attributes. 


\subsection{Integration of Model and Data}

The information gathered during system analysis is used not only for development of the simulation model, but also for several other tasks of the simulation process (as defined in section 2.1.3). In this section two of these tasks will be covered: preparation of model deployment and simulation post-processing. Together with actual model utilization (i.e. simulation), they constitute the three major deployment phases:

1. Pre-deployment phase check if all preconditions are met, alternatives and workarounds if not

2. Deployment "classical" utilization of simulation model

3. Post-deployment phase analysis of results (statistics, etc.), visualization and presentation

The second phase (simulation) is not covered in this thesis. It is obvious that it is a core-competence of simulation and modeling science, such as verification and validation or parametrization of the model, whereas the focus here is set on integration of simulation.

\subsubsection{Model Deployment \& Post-Processing}

\section{Modes of Operation}

Assuming that simulation projects are not initiated as ends to themselves, the intention behind them is reaching certain goals or completing/assisting specific tasks. In such a case the nature of the goal or task will determine the way in which the simulation model is to be used. As sketched in Emrich et al. (2012), the type of use can be categorized into following modes of operation:

1. frequent recurrence,

2. infrequent recurrence and

3. non-recurring.

Frequent recurrence (1) classifies applications in which the simulation model is being used on a regular (e.g. hourly, daily, weekly) basis to generate information, which is immediately put into use by the surrounding system for its day-to-day operations. An example for such a case would be simulation of (lecture) room booking strategies and their immediate evaluation in order to choose the most suitable one for the current booking period. The second mode of operation, infrequent recurrence (2), is one, where the model is being put to use on a regular basis, but in infrequent intervals. In this case the results obtained are more of an informative nature and used for strategic planning instead of operational decision making. This would, for instance, be the case if historical (or forecast) data would be used to test and compare different management strategies in order to improve future decision making. Non recurring (3) applications can also be understood as "consulting assignments". In such projects the goal is to answer a single question or a set of questions, usually in order to assist 
decision making processes. Temporally seen the simulation is used punctually; the model becomes "useless" as a certain process stage is reached. In practice such a job could be to assist a building owner in finding a suitable layout for a given building, based on its projected user structure and behavior.

With closer examination it becomes obvious that these three modes of operation require different levels of integration within the surround system. The reasons for this are mainly found in models' preconditions and economic aspects. The former is explained by the fact that mode (1) deployment requires fast delivery of (sensible/helpful) results. This can only be achieved if a simulation assignment can be processed without, or with only minimal, preparation. Subsequently input data, and parameters have to be readily available as well as all model preconditions (constantly) met. Further, dissemination of results has to be a (highly) automated process. In general complete manual analysis and post-processing of simulation data is too time consuming to provide the stakeholders concerned with the necessary information on time. From an economic perspective it does not make sense to embed a model deeper into a system than necessary. Especially not if this requires to modify existing business processes. Mode (3) projects, for example, are punctual not only regarding the temporal perspective, but usually also stakeholder-wise (i.e. the simulation outcome supports a specific stakeholder with information, assisting her decision making). Hence simulation outcome will probably be used only once ${ }^{15}$ and by a limited group of people. In such a case it is neither sensible nor necessary to come up with (costly) general solutions (e.g. automated data-interfaces \& postprocessing) if a special solution, consuming less resources, is sufficient. This can, of course, imply manual processing of results, which takes more time than an automated solution would. It is this trade-off between resources available and necessary depth of embedment, which directly links to the models capabilities, that is in the focus of this section.

\section{Model Preconditions}

As explained above, the limiting factors are the models' preconditions and economic aspects. These aspects are the key to the question whether a simulation model can be deployed successfully (i.e. in a manner that it reaches the project goals at reasonable "costs") or not. And they are connected closely to another, as the question may be paraphrased as "how much resources are necessary to satisfy the preconditions for successful model deployment?". The answer to it lies within the information gathered during system analysis.

One the one hand, this information is likely to have an influence upon model implementation. Nevertheless a good portion of the model's specifications and its layout are derived from the goals that it has to achieve and the answers to deliver. Subsequently the model's preconditions might differ from what the system provides. Thus the question arises whether the actual system satisfies the preconditions of the model developed. At this point the information from system analysis comes into play again. Comparing the state of the system (i.e. the information from system analysis) with the model's preconditions is much like working though a checklist. Taking a model ready for use (parameterized, validated and verified) its requirements can be classified as:

\footnotetext{
${ }^{15}$ In terms of "at one stage of the project" for which the simulation model has been commissioned.
} 
- structural preconditions and

- data preconditions.

Data preconditions are more or less self-explanatory. They comprise all technical model input (e.g. necessary parameters and data for respective parameterization, as well as all input data) - even if this information is based on expert knowledge and thus not more than an educated guess. Structural preconditions on the other hand include the "soft factors" around these technical sources. They are closely linked to the above defined modes of operation. As previously described, the system/model interfaces will depend on the necessary mode of operation. And the processes that make available the input data necessary are described by the structural preconditions. Without these processes in place it may be possible to produce input data satisfying the preconditions. But it would require additional intermediate steps (such as time consuming manual preparation) which would hinder the simulation from being used in the intended manner.

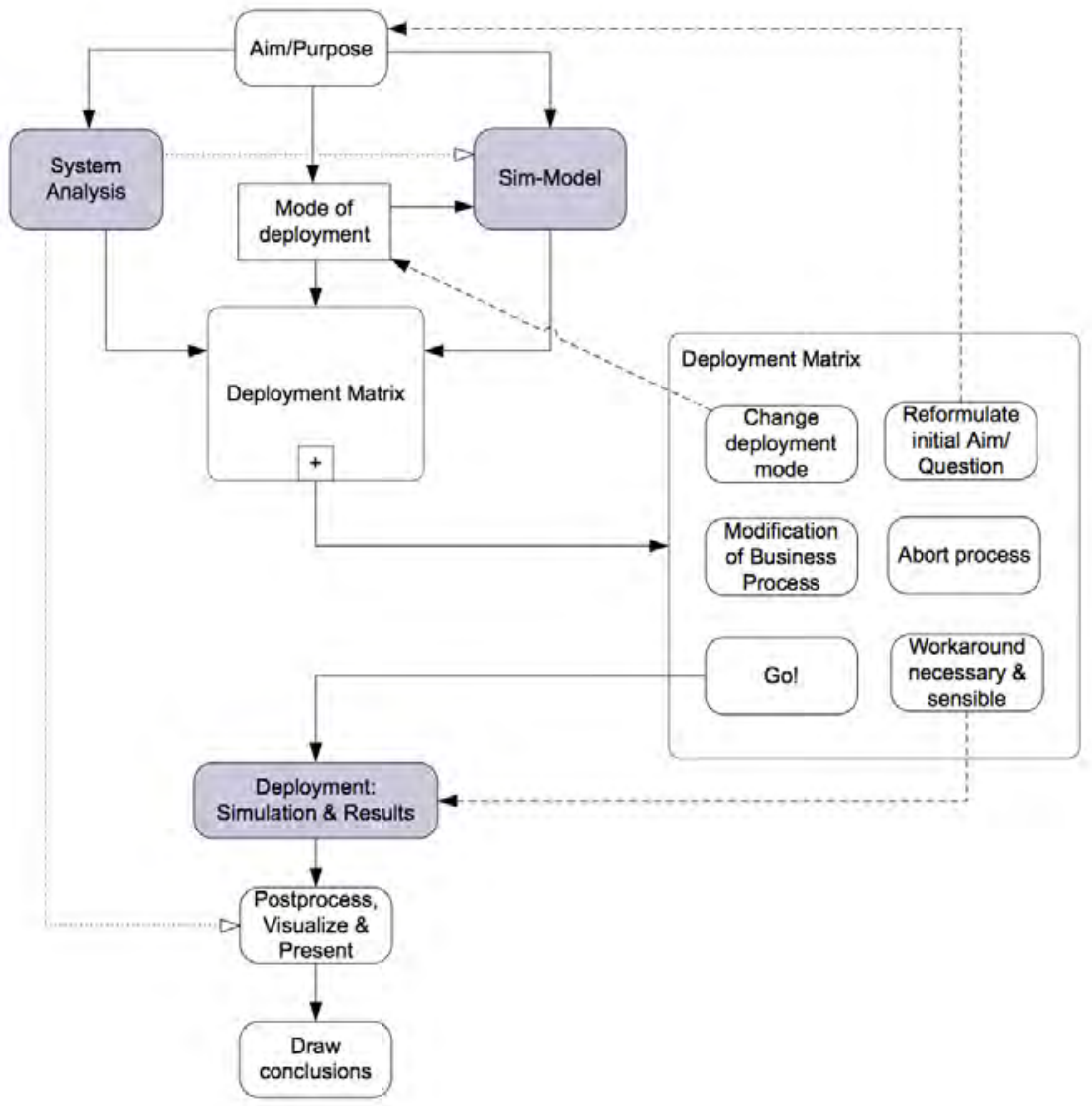

Figure 3.13: Interactions of process steps leading to the deployment and utilization of a simulation model.

Figure 3.13 illustrates the links between system analysis, modes of deployment and the simulation model within the deployment process. As visible the 
(initial) purpose of the simulation project influences system analysis and the simulation model, the latter directly and indirectly. The purpose of the model is of course translated into direct instructions towards the models capabilities. In addition the aim implies a certain mode of operation, via which the model is affected a second time. The link between system analysis and simulation model represents the system-analysis-information, which is used for modeling.

The attentive reader might already have noticed parallels between the methods applied during system analysis (business process and entity relationship model) and the (structural and data-) preconditions. With these it is possible to use the information gathered during system analysis as counterpart to the model's preconditions. To a large extent the ERD will serve as a guideline to whether all of the model's data-preconditions can be met and the BPM in order to check up on the status of the structural ones. If this assessment yields a positive result (i.e. all preconditions are satisfied) then it should be possible to successfully deploy the model. If, on the other hand, the assessment comes up with shortcomings, solutions for these have to be found. At this point the deployment matrix (see Fig. 3.13 and table 3.3) comes into play.

\section{Deployment Matrix}

Good simulation models follow Albert Einstein's credo "as simple as possible, but not any simpler". I.e. they only incorporate the relevant parts (entities, features, etc.) of the system in order to produce realistic system behavior while maintaining a "slim" model. Assessment of those relevant parts is one of the key competences when creating a good model. Further the relevant parts are highly dependent on the purpose of the model. When modeling room utilization, for example, this would require to model the rooms with specifications and features of importance to space-management, such as capacity, technical equipment available or accessibility for handicapped. On the other hand it will most likely not make sense to incorporate room height, thermal insulation and heat flow through walls - which would be necessary for simulation of room climate.

Model preconditions are deduced from the implementation and thus from its features. Hence, as a good model itself cannot be reduced any further, so cannot its requirements and thus its preconditions. As explained above, business process models and entity relationship models can be used to check whether ...

- the preconditions of the model are met,

- the business process of the peripheral system is adequate, and

- if the necessary (input) data can be provided by the stakeholders (identified via stakeholder analysis and in the BPM).

If the requirements cannot be fulfilled, the model cannot be deployed as intended. Instead of aborting the project, in such a case it is also possible to pursue two alternative strategies:

1. Reformulation of the question towards the model in order to abate preconditions.

2. Adaption of the system's business processes. 
Table 3.3: The deployment matrix as a guideline to the feasibility of modes of operation.

\begin{tabular}{|c|c|c|c|}
\hline \multirow[b]{2}{*}{ Preconditions } & \multicolumn{3}{|c|}{ Recurrence } \\
\hline & frequent & infrequent & non-recurring \\
\hline all met & possible & possible & possible \\
\hline some met & $\begin{array}{l}\text { not possible with- } \\
\text { out transformation }\end{array}$ & $\begin{array}{l}\text { eventually with } \\
\text { workaround, else } \\
\text { transformation }\end{array}$ & $\begin{array}{l}\text { likely, based on } \\
\text { workaround }\end{array}$ \\
\hline none met & $\begin{array}{l}\text { not possible with- } \\
\text { out transformation }\end{array}$ & $\begin{array}{l}\text { not possible with- } \\
\text { out transformation }\end{array}$ & $\begin{array}{l}\text { unlikely. transforma- } \\
\text { tion is not reason- } \\
\text { able \& workaround to } \\
\text { costly }\end{array}$ \\
\hline
\end{tabular}

Adequate reformulation of questions, leading to a less demanding model means that the original questions will remain unanswered — which will often be undesirable. If on the other hand changing the business processes is too costly (especially in relation to the expected gain of the simulation project), a compromise between the two options might become interesting. As previously noticed, the depth of integration is connected to the model's levels of detail and its requirements, which hints that changing the mode of operation might open the option for model deployment. Naturally such a change influences the quality and/or amount of information derived from the model. The converse argument holds as well: different simulation goals can be achieved through differing levels of system integration.

The deployment matrix in table 3.3 sums up the possibilities under which a model, depending on the intended mode of operation and the preconditions satisfied, can feasibly be deployed. It is obvious that in case of all preconditions being met it is possible to deploy respective models in all three operational modes - frequent recurrence, infrequent recurrence and non-recurring. With met preconditions decreasing, deployment becomes increasingly problematic. Nevertheless it depends on the level of integration whether unfulfilled preconditions turn out as deal-breaker or not. This is the case for deployment within frequent recurrence scenarios, as these situations require a very close connection between the surrounding system and the simulation model, workarounds to overcome shortcomings on the part of the preconditions will hinder the workflow. Hence the solution will be to either change the mode of deployment, shifting to a less-embedded one (infrequent recurrence or non-recurring) in order to reduce the model's demands, or to transform the actual system. The latter might require simple steps such as setting up a new database-interface. But it could also call for controversial or critical steps such as changing complete business processes, forcing employees to change their routines - including employee resistance against such plans.

When faced with incomplete preconditions in cases of infrequent recurrence 
deployment, finding workarounds can present an option. Depending on the $\operatorname{costs}^{16}$ it can make sense to institutionalize the workaround. In non-recurring applications the model's requirements will, more often than not, be met by workarounds. By definition it is not the intention to integrate the model within the system, thus the necessary information (model input) is assessed only once or a few times. Subsequently it depends on the general availability of this input information and the costs to gather it, whether the model can be put to use or not.

In the case that such information is not available at all, the mode of operation becomes irrelevant, as deployment does not make sense at all. But the more frequent a model's intended use, the more likely it becomes that a transformation of the surrounding system pays off. As necessary model-input - at least parts of it - also presents valuable information for the systems management ${ }^{17}$, its (complete) non-existence acts as a strong indicator for major errors within the business processes in place. Here a change of business processes will most likely be not only of benefit to the model deployment.

Following the deployment matrix the possible options (also see Fig. 3.13) are either

1. a "go",

2. finding a workaround in order to overcome requirement-shortcomings,

3. the necessity to transform the system (change business processes),

4. a change of the mode of deployment, which will usually require

5. reformulation of the initial aim and thus of the questions towards the model, or even

6. abortion of the simulation process.

Thus, in cases one to five simulation is (potentially) possible. Although decision makers will have to judge in cases two to five whether it is worth to pursue the project or better (cheaper) to abort it. If finally the model is being deployed it is going to produce results which, as indicated in Fig. 3.13, have to undergo post-processing.

\section{Post-processing}

The output of simulation models can be as manifold as there are questions towards the model or applications for simulation. While it can happen that a question is answered seemingly straightforward (e.g. 99\% of the time five counters will be sufficient) it will so only for a minority of cases. Staying with the 5-counter-example, a manager could be content with the answer. If she were presented with more information (e.g. in $85 \%$ two counters are sufficient), she would probably think differently and start estimating whether it is worth or not to save $60 \%$ on counters and risk for waiting customers to turn angry. It is thus

\footnotetext{
${ }^{16}$ Cost are not to be understood exclusively financial but in a very general way as "the amount of resources necessary". In businesses this will most often be directly linked to money (e.g. working time of employees), but this does not have to so necessarily.

${ }^{17}$ This is explained by the fact that models have to incorporate all relevant parts and features of the system, which in turn should be known to the management.
} 
of vital importance to the decision makers (or stakeholders) what information is presented to them. This holds especially true the more data a simulation run generates, as it is not about presenting big data but deriving meaningful insights from this data.

Post-processing is to be understood as a decision making support. Thus it is about selecting the relevant data and information in order to generate and present the maximum amount of knowledge. The underlying procedure is best described by the "Visual Information Seeking Mantra" from Shneiderman (1996):

Overview first, zoom and filter, then details-on-demand Overview first, zoom and filter, then details-on-demand Overview first, zoom and filter, then details-on-demand ...

Overview first, zoom and filter, then details-on-demand

Overview first, zoom and filter, then details-on-demand

While this mantra is often used in the context of interactive visualization tools that allow the user to analyze and manipulate data herself, it also serves as guideline for post-processing in Fig. 3.13. As visible in that diagram information from system analysis finds its way into this project stage as well. It is used, along with the initially formulated questions towards the simulation model, to assess the information required by the stakeholder(s). Hence it is not only about what information is presented, but about who it is intended for. Subsequently, if there is more than one group of stakeholders, it might be necessary to present different aspects or perspectives of the data.

The techniques that are to be applied to do so, vary and are depending on the data, the stakeholders and the purpose/the questions that are to be addressed. Thus the decision how to proceed and which tools or methods to apply is a situational one. Moreover, besides understanding what information is to be presented to whom, it requires (at least basic) knowledge of information visualization (InfoVis). InfoVis covers the how of data presentation. Concluding, post-processing has a three-part objective. Its quest is to present meaning- and insightful data (what) to the respective stakeholder(s) (who) in an adequate manner (how).

\subsubsection{Information Visualization}

The field of information visualization (InfoVis) is a very broad one. Subsequently tracing back its origins strongly depends on the definition of infoVis. Geographic maps, which according to Tufte (1986) have first been created almost 7000 years ago, can be understood as very abstract representations, where information is encoded in 2D-space via its coordinates (latitude and longitude). Nevertheless, it took humanity until the middle of the 17th century to encode other than purely geographic information with respect to their coordinates ${ }^{18}$. To fully grasp the potential of positioning values other than cartographic coordinates on a "map", i.e. replace latitude and longitude with other units of measurement (such as time, money, temperature or economic measurements),

\footnotetext{
18 "One of the first data maps was Edmond Halley's 1686 chart showing trade winds and monsoons on a world map." (Tufte, 1986, p. 23).
} 
took another 100 years, with an exceptional - and isolated - first time-series plot $^{19}$ reputedly produced in the tenth or eleventh century.

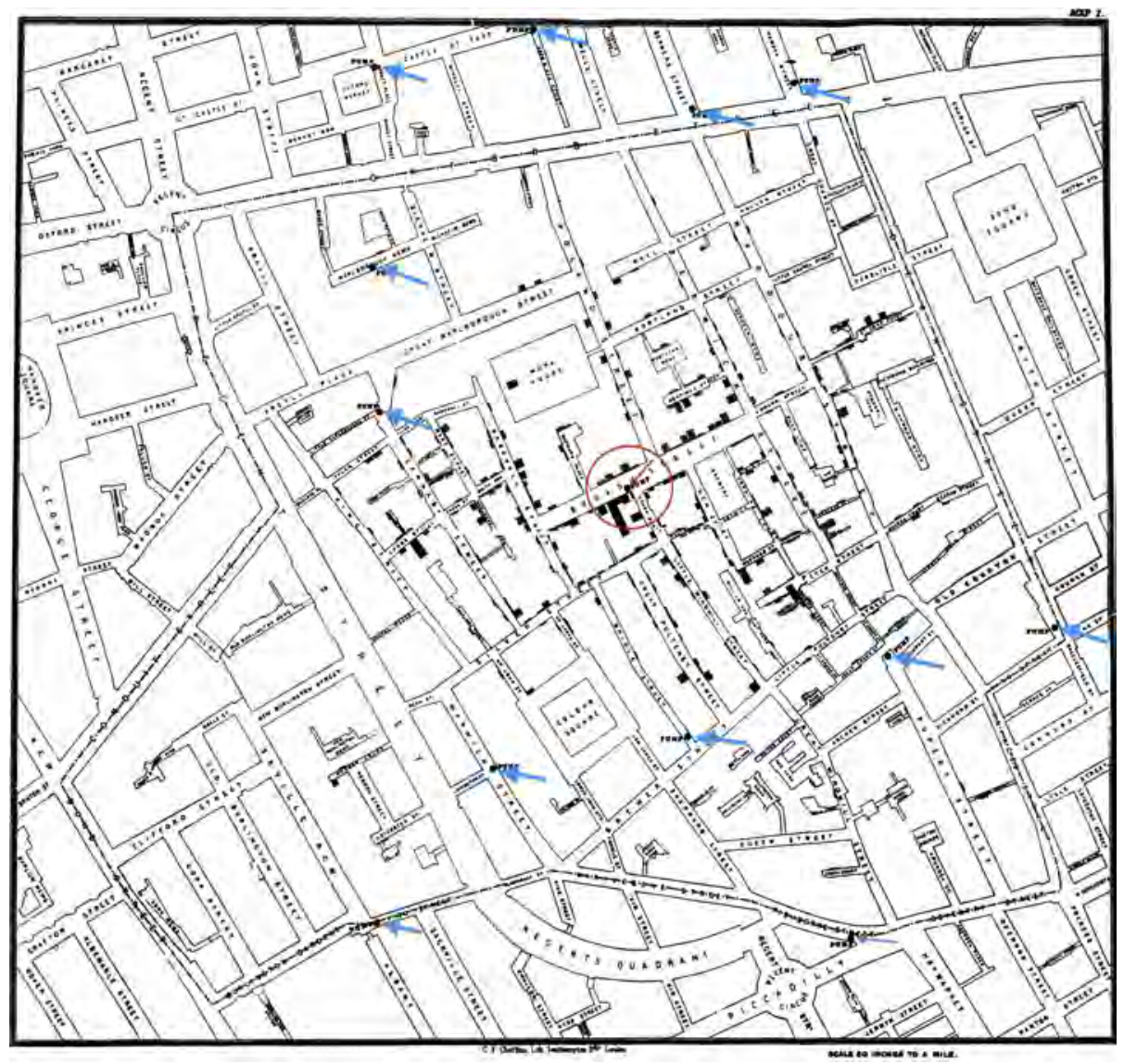

Figure 3.14: John Snow's map plotting cases of deceased cholera victims (stacked black dashes; appear as bars due to resolution) and the location of water pumps (blue arrows), leading to disablement of the Broad Street pump (encircled red arrow) and the end of the cholera epidemic ${ }^{20}$.

Staying with data maps, two early and well known examples for the combination of maps and non-geographic data are the cholera-epidemic map of Dr. John Snow (made in 1854) and that of Napoleon's Russian campaign drawn by Charles Joseph Minard (in 1861). Especially Snow's map (depicted in Fig. 3.14) is a great example for exploratory data analysis. At the time Snow designed his map, the cause for cholera was widely believed to be "miasma" 21 — instead of germ-polluted drinking water. Although Snow suspected that water was functioning as vector, he could not affirm this theory by analysis of the water. But

\footnotetext{
${ }^{19}$ The time-series plot, depicting "the inclination of the planetary orbits as functions of time" (see Tufte, 1986, p. 28), although with a faulty or confused content, appears some 800 years prior to following time-series plots.

${ }^{20}$ Image is in the public domain due to its age; author: John Snow; obtained via WIKIPEDIA: http://en.wikipedia.org/wiki/File:Snow-cholera-map-1.jpg last checked: September 16, 2013.

${ }^{21}$ Which was "bad" of "foul" air, with the name derived from ancient Greek $M \iota \alpha \sigma \mu \alpha$ for "pollution".
} 
his map showed that the cases of cholera related deaths were clustered around a single water-pump in Broad Street. This eventually led to the pump being disabled, the cholera epidemic contained and contaminated water being - albeit slowly - accepted as carrier of cholera.

According to Keim et al. (2010) the shift from "confirmatory data analysis (using charts and other visual representations to just present results) to exploratory data analysis (interacting with the data/results)", as foreshadowed by Snow, was "one of the most important steps" to bring together the fields of statistics and visualization. Although this step was undertaken by John W. Tukey in his 1977 book ("Exploratory Data Analysis"), it was not before computer graphics became (more) powerful that the emphasis on visualization picked up — which happened at the end of the 1980s.

Even though InfoVis is primarily about visualization and not about computing, there is a fair amount of it required in the journey from raw data to a visual representation. This holds especially true when one is to visualize big data. The process from (raw) data to a visual representation, which is depicted in Fig. 3.15, starts with structuring the raw data at hand (i.e. organize data in tables or data-bases). This is necessary in order for the data to be (computer) processible. In this step the user may already choose to focus only on a part or certain aspects of the data. Previously mentioned visual information seeking mantra comes into play at latest in the next processing step: creating a visual mapping. This step takes the data (of interest) and puts it into a visual form, thus designing a visual structure. It is arguably the most important step when creating an InfoVis-graphic, as the next step (view transformations) only alters what has been produced so far. Common examples for view transformations are scrolling or changing the view angle of 3D-visualization. As visible in Fig. 3.15, user-interaction is intended at every step of the process. It is thus desirable that the whole process is an interactive one, as it allows the user to play with and explore the data. Preferably the visualization-tool allows to interactively filter and zoom (e.g. brushing or dynamic queries) within the graphical representation - a task that otherwise (at least filtering) has to be done when structuring data or creating a visual mapping. The possibility of "playing around" and exploring data with such a powerful visualization tool can result in unexpected insights, which lead Shneiderman to the statement that good "visualization gives you answers to questions you didn't know you had". Yet, developing a visual mapping which is adequate for the data given and able to convey the intended messages requires to understand the human process of visual perception.

\section{Visual Perception}

It is no coincidence that information visualization is relying on visual perception and not on another of the human senses. The reason for this is the immense "bandwidth" of information that can be picked up, transmitted and processed visually. As explained (in greater detail) by Few (2004), this procedure involves several actors. First, "let there be light" (TLB, 1971, Genesis 1:3). For without light the process won't work. Light functions as medium conveying information from an object into the eye. The eyes' sensors then translate the incoming light into neural signals which are transported into the brain via the optical nerve. Finally the brain processes these neural signals, transforms them into a picture and immediately starts to link it with associated information. It is at the end 


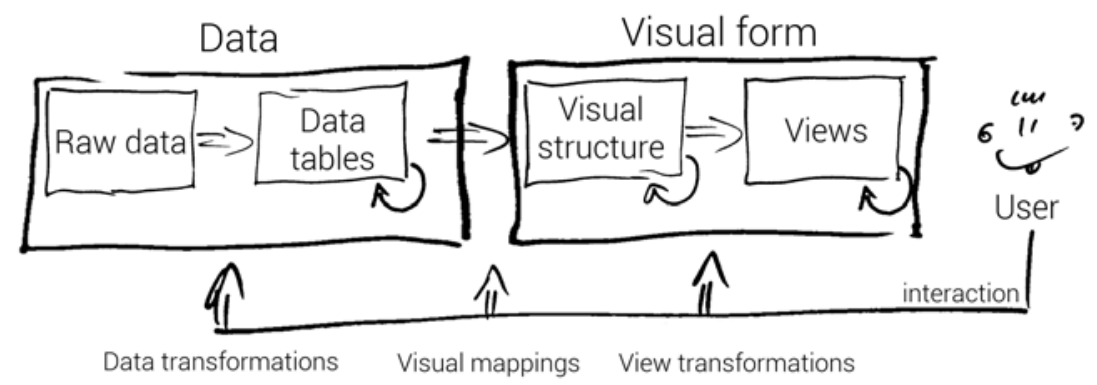

Figure 3.15: The process of turning raw data into a visual representation, with its feedback loops for user/human interaction.

of this cognitive process - from a physical stimulus to its interpretation - that the essential part of information visualization kicks in.

The brains image processing is taking advantage of three types of "memory":

- iconic or sensory memory ${ }^{22}$,

- short-term memory and

- long-term memory.

When it comes to (good) visual design, the iconic memory plays the most important role. As described in Mazza (2009), it retains impressions from the sensory organs only for 250 to 500 milliseconds. Further, it stores visual information automatically and without conscious control. For this reason the processing done by the iconic memory is also referred to as preattentive processing. In this process "only a limited set of visual attributes is detected. Such basic features include colors, closure, line ends, contrast, tilt, curvature and size [according to "Preattentive processing in vision" by Anne Treisman, 1985]." (Mazza, 2009, p. 34).

The short-term memory receives a limited amount of information from the sensory memory. It remains there for a limited time (ranging from a few seconds up to a minute). An important aspect of the short-term memory is its limited storage capacity. In contrast to sensory memory the short-term memory "is conscious, and involves an attentive process of perception" (Mazza, 2009, p. 34). As argued by Miller (1956) the limit for (equal) stimuli that can be remembered by the short-term memory lies around seven ${ }^{23}$. This capacity can be increased by organizing information into chunks (e.g. several short number-blocks of a longer phone number).

Only information that makes it into the long-term memory is stored for longer periods of time (potentially up to a lifetime). Transfer of information from short- into long-term memory (usually) relies on rehearsal and repetition. The information in this part of the memory "is vitally important to visual perception

\footnotetext{
$34)$.

${ }^{22}$ The visual sensory memory is usually referred to as iconic memory (see Mazza, 2009, p.

${ }^{23}$ Although there are exceptional outliers, such as "musically sophisticated person[s] with absolute pitch [who] can identify accurately any one of 50 or 60 different pitches" (Miller, 1956) instead of the average five to nine.
} 
because it holds our ability to recognize images and detect meaningful patterns, but we don't have to understand very much about long-term memory to become better designers of tables and graphs" (Few, 2004, p. 97).

Visual perception, and with it our preattentive capabilities, are evolutionary products shaped by the natural selection-process, the survival of the fittest. Thus they are geared towards fast identification of important, potentially lethal, albeit small, deviations of the "norm" - and adequate response. This is the reason why preattentive attributes "pop out" (Ware, 2004) at the viewer. When it comes to presenting information visually, it is the address of these preattentive processing-capabilities that can determine the quality and informative value of a graphic or visualization.

\section{Preattentive Processing and Gestalt Principles}

A great example for the power of preattentive processing and its advantages (subconscious, automated and fast) over attentive (conscious, sequential and much slower) processing is identification and counting of all instances of a given number (in this case the number 2) within a large block of numbers.

\section{6 \\ 19024641106119241470443887313514781304003820467367 \\ 43605550551134767340206493682956765115437758900563 \\ 93956954954613997134585138683997107984408058086138 \\ 33169384513389710884286434601145334610601070743191}

Figure 3.16: Finding all instances of "2" requires - slow - attentive processing.

In Fig. 3.16 the reader has to apply attentive processing which will, depending on her skill, take at least half a minute if not well over one minute. In Fig. 3.17 on the other hand, she can rely on the support of preattentive processing. With this support, the task of identification is accomplished within split seconds and counting within a few seconds.

\section{6 19024641106119241470443887313514781304003820467367 43605550551134767340206493682956765115437758900563 93956954954613997134585138683997107984408058086138 33169384513389710884286434601145334610601070743191}

Figure 3.17: Identification of all 2's is done preattentively and fast.

In the second figure, it is possible to identify 7 out of 250 numbers within split-seconds - with the aid of preattentive processing. Taking this one level further, as explained in Ware (2004, p. 145), the human brain can scan a $500 \mathrm{x}$ 500 array of white pixels in search of a single black one within one second. Thus it is possible to replace the 250.000 pixel screen every second and subsequently conduct a search of 15 million pixels within a minute. As noted by Ware himself, this is an arbitrary and very simple example, yet it does give an idea of the power of preattentive processing. 
According to Ware preattentive properties can be grouped in four categories: form, color, spatial position and motion. Examples for attributes of form are found in Fig. 3.19 and for color in Fig. 3.20a (intensity) and Fig. 3.17 (hue). While these attributes are readily registered by the human brain, the challenge is to effectively transport information with them. This becomes evident in Fig. 3.18, where the same ratio (2:1) is represented once by line length (Fig. 3.18a) and once by the area size of circles (Fig. 3.18b). It is apparent, that not every attribute is equally suitable for every type of data. Thus, in order to choose the appropriate attributes, one has to identify what sort of data is to be conveyed. Generally speaking data can be of categorical, ordinal or quantitative nature. Quantitative (or numerical) data can be compared naturally (e.g. a list of numbers), ordinal data is not of a numeric nature but does have an intrinsic order which allows for sorting (e.g. the quarters or months of a year). Categorical data on the other hand lacks intrinsic order and thus its sorting is purely arbitrary (e.g. a list of countries could be sorted by alphabetical order, by number of letters in their names or by continent). In table 3.4 (on page 77 , source: Mazza (2009)) preattentive attributes of all four categories are classified according to their suitability for representation of these three types of data.

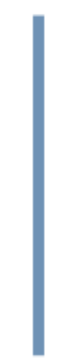

(a) Line length is optically perceived very accurately - the ratio is identified easily.

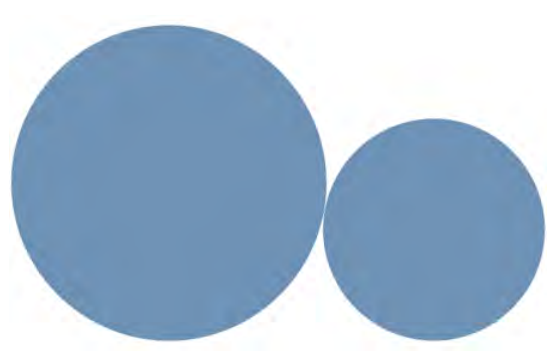

(b) Area size is perceived very inaccurately; not suitable for comparison of numeric values.

Figure 3.18: Comparison of preattentive attributes (length and area size) to carry information; both diagrams represent a 2:1 ratio.

While no attribute is suitable for all types of data, some attributes (such as hue or flicker) are not suitable (at all) for any type. Nevertheless they can be used for other visual purposes. Flicker for example is an extremely eye-catching attribute. It is therefore predestined to be used when very important/critical information has to be made stand out (e.g. pointing out malfunctioning of cooling on nuclear power plant dashboard, also see Few (2006)). Others can be used to point attention towards certain aspects of data and hereby tell a story (e.g. using a contrasting hue to emphasize the bar of an important event in a bar chart).

Conscious use of preattentive attributes in visualizations can greatly improve them. But there are limits to their use. As previously written, humans can distinguish only around 7 equal stimuli. Thus a diagram depicting 12 differently encoded data-sets (e.g. time-series of stock prices for 12 companies, each 


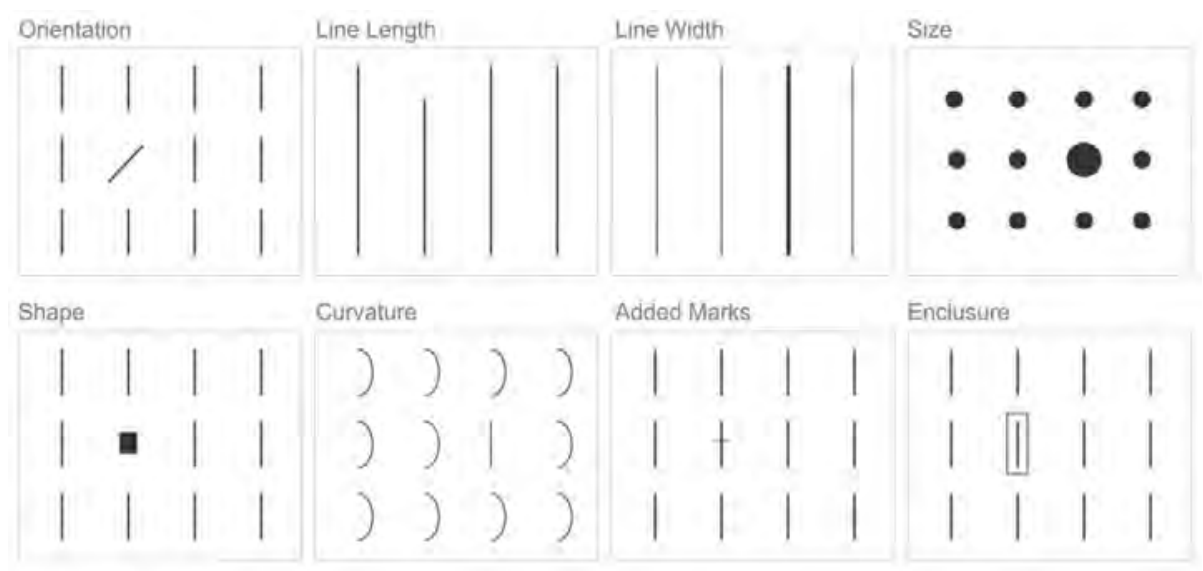

Figure 3.19: Selection of preattentive attributes of form (source: Few (2004, p. 99)).

company's time series encoded with a distinct color ${ }^{24}$ ) will most likely require the viewer to switch between the actual diagram and the explanation (legend) several times.

Similar holds true when trying to combine several attributes, as shown in Fig. 3.20. When using a single attribute (color intensity, left image) to encode objects, the three groups can be distinguished easily. When adding a second attribute (shape, right image) it is still possible to preattentively distinguish the object-groups for both attributes. In this case identification of all black, medium or light gray objects - regardless whether they are circles or squares. But it is not possible to preattentively pick out the combined groups (e.g. light-gray squares).

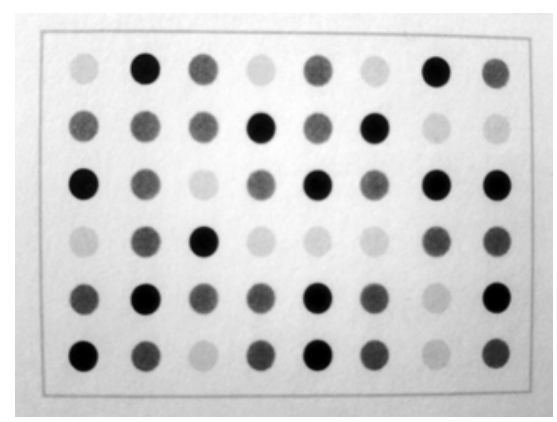

(a) Use of color intensity (black, medium gray and light gray) to classify three groups.

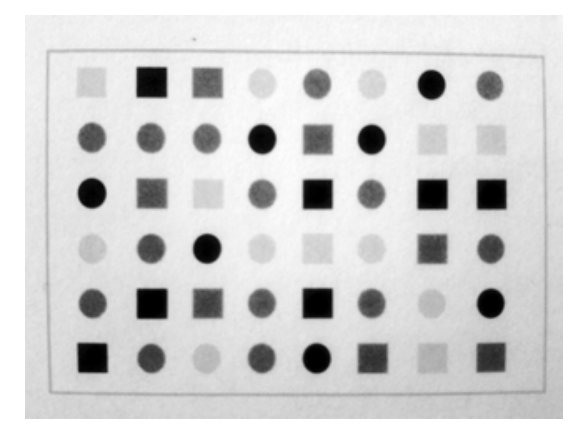

(b) Combination of color intensity (3 groups) and shape (2 groups) to encode six groups of objects.

Figure 3.20: Combinations of preattentive properties cannot be picked out preattentively. Differentiation is only possible for one attribute at a time (source: Few (2004, p. 107)).

Further, the power of preattentive attributes diminishes as more of them get

\footnotetext{
${ }^{24}$ In addition to the limits of the short-term memory, this example also stresses the availability of well distinguishable colors which will look good on a screen and when printed.
} 
Table 3.4: Preattentive attributes and their suitability to encode types of data ( $\checkmark$ suitable, - limited suitability, x not suitable), according to Mazza (2009, p. 40).

\begin{tabular}{lccc}
\hline Attribute & Quantitative & Ordinal & Categorical \\
\hline Color & & & \\
Hue & x & x & $\checkmark$ \\
Intensity & - & $\checkmark$ & x \\
Form & & & \\
Orientation & - & - & x \\
Length & $\checkmark$ & - & x \\
Width & - & - & x \\
Size & - & - & x \\
Collinearity & x & x & x \\
Curvature & - & - & x \\
Spatial grouping & x & x & x \\
Added marks & x & x & $\checkmark$ \\
Shape & x & x & $\checkmark$ \\
Numerosity & $\checkmark$ & $\checkmark$ & x \\
Spatial position & & & \\
2D position & & & \\
Stereoscopic depth & x & x & x \\
Concavity/convexity & - & - & x \\
Movement & & & \\
Flicker & & & \\
Motion & x & x & - \\
\hline & - & - & x \\
\hline
\end{tabular}

used within the same visual representation. Ware (2004, p. 152) described this as follows:

It is easy to spot a single hawk in a sky full of pigeons, but if the sky contains a greater variety of birds, the hawk will be more difficult to see. Studies have shown, that two factors are important in determining whether something stands out preattentively: the degree of difference of the target from the nontargets, and the degree of difference of the nontargets from each other (Quinlan and Humphreys 1987; Duncan and Humphreys, 1989).

This quote also indicates the answer to the question which attributes are stronger in terms of better processing - there is no real answer. The salience of attributes depends on the feature's strength and the context in which it is used. 
Figure 3.21: The phenomenon of gestalt principles: A combination of one triangle and three rectangles is naturally recognized and interpreted as a house (source: Mazza (2009, p. 41)).

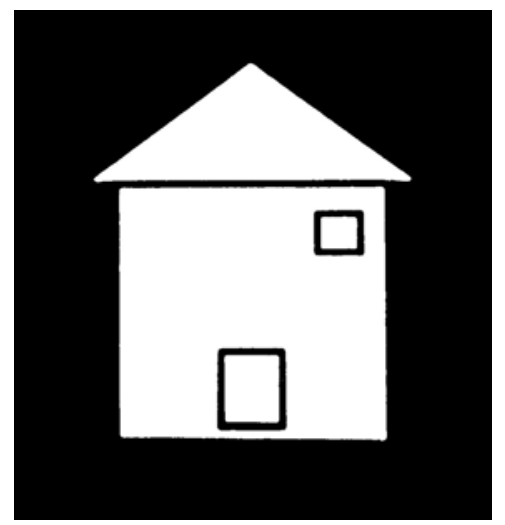

Besides preattentive processing the gestalt principles are major contributing factors that determine "what we see". The term is derived from the German word "Gestalt", which translates as "shape" or "form". It describes the phenomenon that we "see" more than there is, as illustrated by the example in Fig. 3.21. According to Mazza (2009); Few (2004) it has been first studied at the beginning of the $20^{\text {th }}$ century (1920 or 1912 respectively). The "basic principle" behind it "is that the whole ... is not simply the sum of its parts ... but has a greater meaning than its individual components" (Mazza, 2009, p. 41). The following is a selection of a few gestalt principles:

- principle of proximity,

- similarity,

- enclosure and

- closure,

- continuity and

- connection.

The principle of proximity describes the effect, that the human brain perceives objects close(r) to each other as forming a group (see Fig. 3.22a), this can even "overrule" preattentive attributes, as shown in Fig. 3.22b. A similar effect of perceiving objects as belonging together do have the principles of similarity (Fig. 3.22c) and enclosure (Fig. 3.22e). These state that objects with a similar "look" (form, size, color, texture, ... ) or objects enclosed by a visual border, respectively, appear as groups. In the latter case the border does not have to be anywhere near dominant, as shown by the subtle ones in the example. The principle of closure (Fig. 3.22d) and continuity (Fig. 3.23a) are closely related and are the reason why we tend to complete forms which are either incomplete or disrupted by some other object. Finally the principle of connection provokes the cognition of objects connected belonging together (Fig. 3.23b). This is put into use in line charts, for example.

\section{Graphical Excellence}

The expression "good visualization" has been previously used a few times without defining what good refers to in this context. And it is worth noting that it 


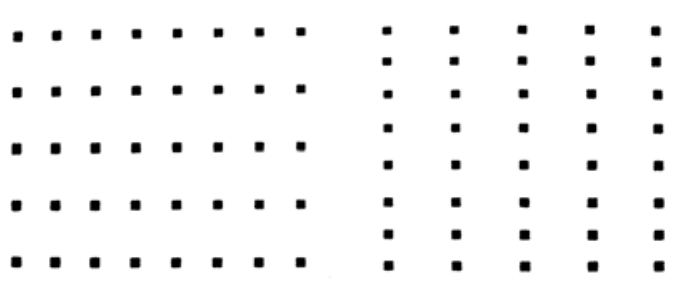

(a) Principle of proximity, depending on spacing the objects are perceived as rows or columns (e.g. used for table design).

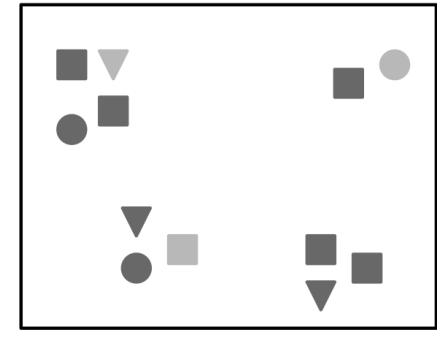

(b) Example of proximity "overruling" preattentive attributes (form and color).

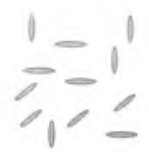

(c) Principle of similarity, similar objects are perceived as groups that belong together.
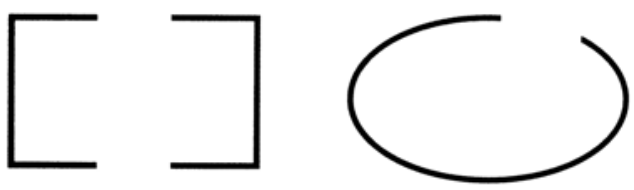

(d) Principle of closure, leads us to complete shapes.
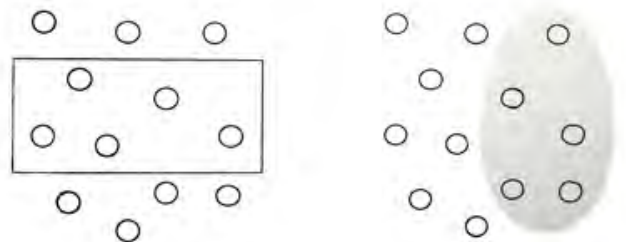

(e) Principle of enclosure, only subtle enclosure is necessary to provoke a completely changed perception of the same arrangement.

Figure 3.22: Gestalt principles of proximity, similarity, closure and enclosure (source: Few (2004, p. 110 ff.)).

is unlikely to find a unanimous definition for something that is (also) subject to taste - and thus to personal preferences. Nevertheless, a lot of work has been done on the subject, of which Edward Tufte's stands out. His work is unarguably one of the most prominent in information visualization, especially with regards to statistical graphics and visual communication of scientific matter. Visualizations are a means of communication and as such they can generally be considered "good" if they manage to complete the task intended. I.e. if they transport the intended information in such a way that the intended recipient gets the intended message. 

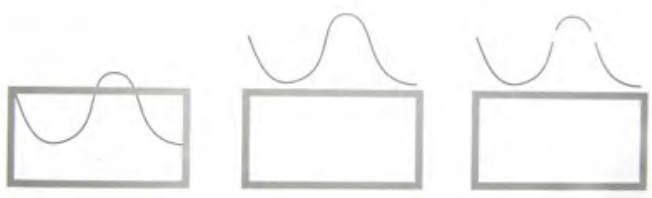

(a) Principle of continuity, gives the impression to see object continued instead of partitioned.

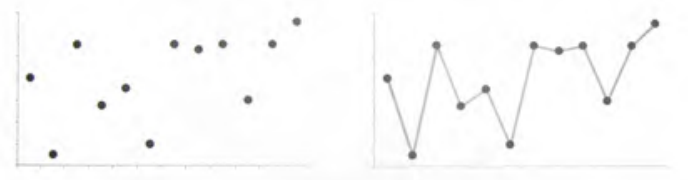

(b) Principle of connection, can be used to put the shape of data into focus.

Figure 3.23: Gestalt principles of continuity and connection (Few (2004, p. 110 ff.)).

Unfortunately, as Mazza (2009) puts it, "there is no magic formula that, given a collection of data, shows us systematically which type of representation to use." Thus it lies with the creator of a visualization to make the best out it. Though a quote by Tufte (1986, p. 51) can serve as a helpful guideline on the way towards graphical excellence:

"Graphical excellence is that which gives to the viewer the greatest number of ideas in the shortest time with the least ink in the smallest space."

As we will see, this sentence puts almost all his guidelines and principles in a nutshell. But let's start with an example of graphical excellence: the (previously mentioned) map of Charles Minard depicting Napoleon's army's devastating Russian campaign (mentioned on 71). This map (Fig. 3.24), as nearly all examples considered being graphically excellent, is multivariate. It shows Napoleon's army's size (including auxiliary troops) - differentiating between the army's advance (brown band) and retreat (black band) - with respect to its geographic position during the Russian campaign (1812). In addition location of important settlements is given and - for withdrawal - the temperature (of an extremely cold winter, given on the Réaumur scale), the rivers crossed and a timescale for those events is given.

An important term that was coined by Tufte is the data-ink-ratio (see Tufte, 1986, p. 93). As its name indicated, he describes with it the amount of ink that is dedicated solely to depicting the data, "the non-erasable core of a graphic, the non-redundant ink arranged in response to the variation in the numbers presented." The goal of a graphic should be to maximize the data ink by removing other ink (labels, grid-lines, etc.) as far as possible. An improved data-ink ratio also contributes to the overall perception of the graphic, as the viewer can identify the important aspects faster. A second approach to improve

\footnotetext{
${ }^{25}$ Image is in the public domain due to its age; author: Charles Minard; obtained via WiKIPEDIA: http://commons.wikimedia.org/wiki/File:Minard.png last checked: September $17,2013$.
} 


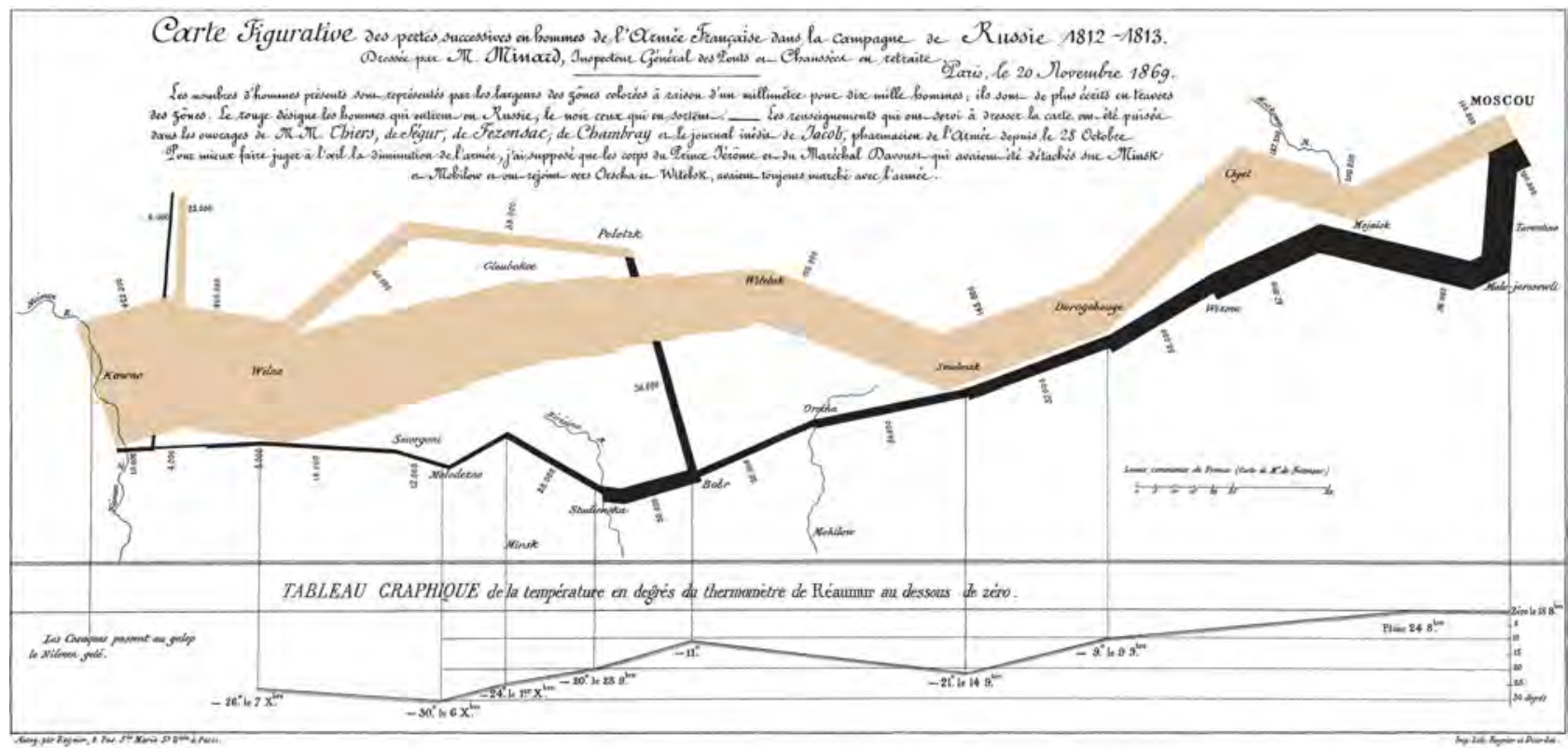

Figure 3.24: An example of graphical excellence: Charles Minard's map of Napoleon's army's Russian campaign ${ }^{25}$.

this ratio is to reduce (unnecessary) redundancy, which is illustrated very nicely by Fig. 3.25. In it the data is displayed by six attributes (height of left and right lines, height of shaded bar, position of top line, position of number and finally the number itself) at the same time - removing any five of which would still tell the same story.

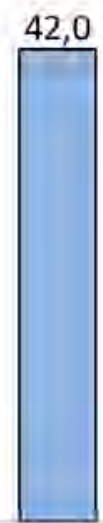

Figure 3.25: Data displayed redundantly by six attributes.

Another technique to improve the data-ink ration (and readability) is to de-emphasize non-data ink (i.e. using very subtle shades for non-core data). Further, to transport a clear message fast, data should be organized and highlighted, as argued by Few (2004, p. 117 ff.). This chapter (General Design 
for Communication) is highly recommended for communication of numerical data, as it goes beyond visualization and into general communication of it (e.g. table design). Finally graphical excellence is not possible without graphical integrity.

Statistics, statistical information and visualizations of such are often greeted with skepticism. Quotes such as "there are three kinds of lies: lies, damned lies, and statistics" 26 or its German counterpart "Never trust any statistics that you didn't forge yourself" 27 are everyday examples of this skepticism. And partially with good reason, as statistics and diagrams were and are often (bee it consciously or not) produced falsely or at least misleadingly.

Graphics and diagrams must not distort the data or put it out of scale. Common examples for such a practice are two-dimensional representations of one-dimensional data. As the univariate (1-D) measurement increases, the 2$\mathrm{D}$ representation does so in both dimensions creating an inaccurate impression as the area is growing quadratically instead of linearly. Even if the graphical representation is scaled properly (i.e. data is represented by the area instead of the edge lengths) its use is sub-optimal as the preattentive attribute area is not well suited for encoding of numerical data (see table 3.4 and section "Preattentive Processing and Gestalt Principles on page 74). If such a graphic is designed conscious of these effects, it is a clear attempt to deceive the audience. But often such faulty graphics are created unconsciously or by accident. The reasons for this often are a lack of "quantitative skills" (Tufte, 1986, p. 79) in the people producing info-graphics, which are usually illustrators with an artistic goal and no (or only little) statistic know-how. Tufte also lists "the doctrine that statistical data are boring" as a reason. Because of it diagrams have to pep up the data that would otherwise be "boring" - through decoration \& chartjunk. Darrel Huff (1977) devoted his whole book "How to Lie with Statistics" to this issue and covers a wide range of techniques on how data and statistics can be used to tell a story (consciously) misleading.

\section{Considerations on Presentation of Data}

Today tools for presenting data and information are virtually omnipresent. Spreadsheet and slide-show software is found on virtually any computer and an increasing portion of mobile phones (smart phones) and other gadgets. They are extremely convenient and (supposedly) state of the art for almost any presentation, independent of the area of application. But this does not necessarily mean that they are put to use in a good way. As with every tool, the easier it is to use, the more people can use it - regardless whether they know what they are doing (with it) or not. The ease of use encourages to experiment with the numerous possibilities, while the results are judged by not goal oriented standards. In the following spreadsheet software and the effect of slide-show programs are discussed (very briefly).

Arguably, Microsoft Excel, besides being the the most powerful and versatile spreadsheet program, it is also the most widely used - and thus de facto

\footnotetext{
${ }^{26}$ Of which the true author is not definitely known, but which was popularized by Mark Twain.

${ }^{27}$ Original: "Traue keiner Statistik die du nicht selbst gefälscht hast". In the Germanspeaking countries it is commonly referred to as being by Winston Churchill, but it is most likely a product of Nazi propaganda trying to depict Churchill as liar.
} 
the industry standard. It is thus no surprise that a vast number of diagrams encountered are produced by it and it shall serve as representative example for a brief analysis ${ }^{28}$. A first glimpse at its standard charts shows that the first five categories (vertical bar charts, line charts, pie charts, horizontal bar charts and area charts) offered are those that can be reasonably used for univariate data (with the exception of ring charts and radar charts, which are packed further down the menu). Taking a look at these five categories (see Fig. 3.26), one notices that all of them offer 3D-visualizations. Moreover, from the 53 options within these five categories only 19 are not 3D! Thus of the options one has to visualize univariate data much more than half (namely $65 \%$ ) are 3Dvisualizations. As projections of three dimensions onto two-dimensional space by definition inherit distortion (or loss of information), the user is offered a vast amount of visualization options that are going to produce inexact graphics.

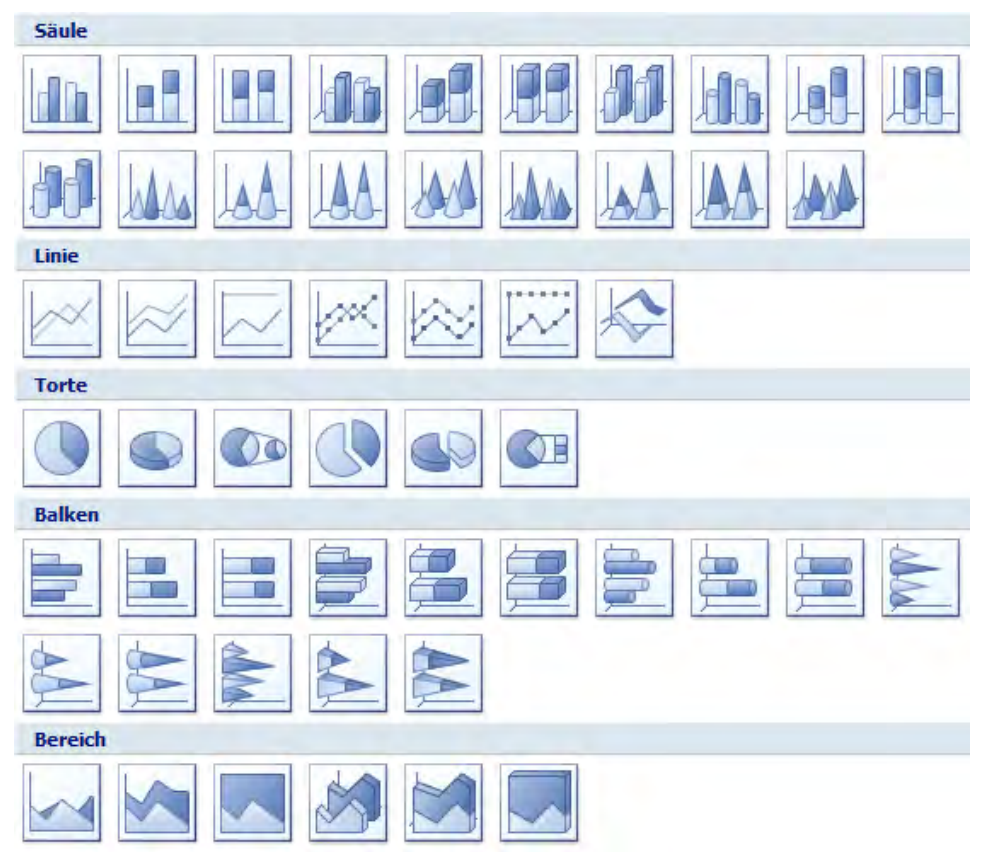

Figure 3.26: Excel's top five chart categories.

Further, pie charts are listed third. As pie charts use angles and areas to encode quantitative data, they should generally be avoided, as these preattentive attributes are ill-suited for this purpose (compare table 3.4). The addition of $3 \mathrm{D}$-distortion to a pie chart (1/3 of the options offered by the software) renders it totally useless, as it becomes impossible to compare angles and areas, especially as the differences in data become smaller and smaller.

Even if sticking to slender visualizations (e.g. bar or line charts), suitable for the data at hand, Excel's default is improvable - to say the least. Figure 3.27 shows drastically the visual clutter that the software uses in its default (line) diagrams and compares it to an improved version - both of which use the same sized area. In the present case improvement was made by deleting

\footnotetext{
${ }^{28}$ Excel 2007 was used in this case.
} 
of grid lines, the de-emphasis of axes, reduction as well as de-emphasized of their labeling and deleting of the legend, freeing space used to display data. Finally the important ${ }^{29}$ data (red line) highlighted, while the other data (used for comparison) serves as an informative background (de-emphasized). Although manual improvement of the data-ink ratio is tedious it is possible - and pays off.

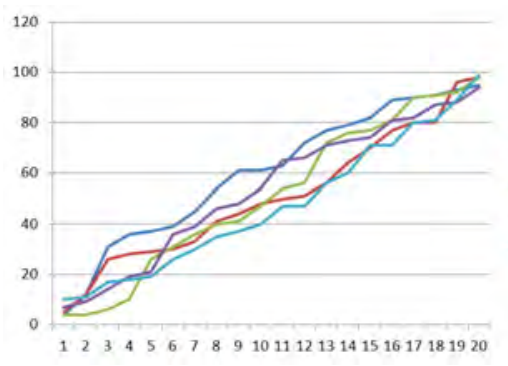

(a) Default line chart ...

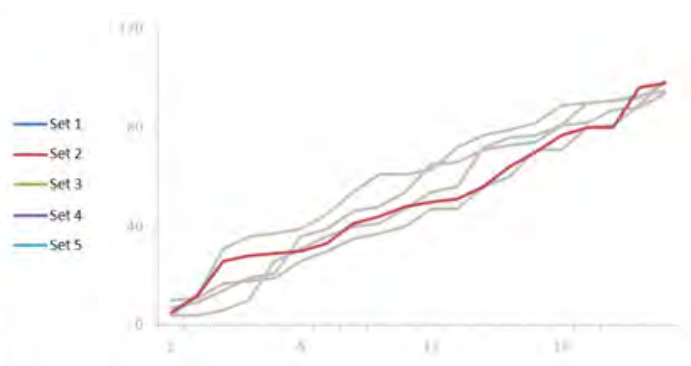

(b) after (manual) data-ink maximization.

Figure 3.27: Comparison of Excel's default line chart and possible (manual) data-ink maximization (data: random numbers sorted ascending).

When communicating messages with data it is not only about poorly designed templates as well as limited and/or tiresome possibilities for improvement. The general suitability of charts to transport this message properly is a critical aspect as well. Choosing the form of visualization requires understanding of the (visual) perception process - as mentioned earlier. For example doesn't it make any sense to present real estate prices of several districts by a line chart. The continuous line would (gestalt principle of connection) mislead the viewer to perceive the values as one evolving value. As the districts do not have any intrinsic order according to which the prices could be connected, a different chart (e.g. bar chart) should be used.

These shortcomings of spreadsheet programs (poorly designed templates and misuse of techniques) can be mapped one to one when talking about slide-show presentations. As argued in Tufte (2006) utilization of slide-show programs is so common that it is hardly ever reflected. In his work he enumerates several major shortcomings of slide-show presentations. Among them (1) bad style sheets, using too much space for unnecessary elements and (2) bullet points which create the impression of structure but at the same time encourage use of incomplete sentences, often lacking the context-relevant verb. Another one is (3) the low data density of slides, which he describes as follows: "People read 300 to 1,000 printed words per minute, and find their way around a printed map, $\ldots$, displaying 5 to $40 \mathrm{MB}$ in the visual field. ... Yet, in a strange reversal, $\ldots$, the PowerPoint slide typically shows 40 words, which is about 8 seconds of silent reading". This low data density holds especially true when displaying tables on slides.

While parts of this work have been criticized for being imprecise or biased (as for example by Doumont (2005)), this does not apply to the core of Tufte's critique: slide-show presentations have to be treated with much care when used

\footnotetext{
${ }^{29}$ As the data is complete bushwah, the "important" data set is an arbitrary choice. It only serves to show the potential of improving chart readability.
} 
for communication of technical content, which is explained by him by the very dramatic example of the Columbia Space Shuttle disaster. In that case slides were passed on across hierarchical levels and departments which diluted the message, as oral explanations were lost and technical terms not specified precisely enough (partially due to the software's inability) ${ }^{30}$.

He concludes that technical papers (or hand outs) are often the better way to convey messages, as they offer the room to elaborate and describe all necessary details of a topic. The same holds true for tables, which often are to be preferred over graphs. Nevertheless, slide-show presentations - when done right (as for example described in Duarte $(2008,2010)$ ) - do have their validity. Again, the most important is to "above all else show the data" (Tufte, 1986, p. 92). Choosing the right method, approach and technique for this task is part art, part science and a lot of practice and experience.

\footnotetext{
${ }^{30}$ It is worthwhile noting that it was also Tufte who showed how the use of bad charts contributed to the Challenger Shuttle disaster several years before.
} 


\subsection{Mathematical Modeling Concepts Applied}

The following three modeling concepts (Discrete Event Simulation, Cellular Automata and Agent-Based methods) are used for the creation of two simulation models referred to by this thesis. The first one was developed in the course of the MoreSpace-project, with the aim to improve room utilization at Vienna University of Technology (see "Genesis of the MoreSpace-Project" and the following). The second model, which is presented in detail in section 4.3.1, was developed with a distinct focus on workplace utilization in office buildings. Both applications rely on a hybrid coupling of the approaches, which is described in the very end of this chapter.

\subsubsection{Discrete Event Simulation}

As the name indicates, discrete event simulation (DES) is an approach for simulation of discrete systems. In contrast to continuous simulation (e.g. in its purest form with electronic analog computers) in such systems time does not advance continuously but in discrete steps. These steps are either of predefined step size or they leap directly from one event to the next. These events can in turn trigger other events or routines, which allows modeling systems with a complex behavior. Thus the main challenge is to keep track of the event sequence (event calendar).

Informally discrete event systems can be characterized (top-down) as follows:

- Discrete event systems are collections of components which are interacting in order to perform given functions.

- Components are represented by discrete state-sets and operations defined upon these sets.

- Operations are mainly specified as set of rules which determine state transitions.

- State transitions are performed over time, but only on occurrences of events.

- Events are classified by their trigger into externally (e.g. arrival of a message) and internal (e.g. timeout) events.

For a formal description of discrete event systems often the discrete-event systems specification (DEVS) is being used. Although the DEVS, as defined by Zeigler (2003), started out as a formalism for modeling of discrete event systems, in the meantime he regards it as "a framework rather than a particular technique, method or technology" (see Zeigler, 2003, p. 160). His gradual development of the DEVS can be traced back to his 1976 book "Theory of Modelling and Simulation" (Wiley \& Sons), although it first crystallized in his 1984 book "Multifacetted Modelling and Discrete Event Simulation" (Academic Press). In the meantime DEVS is a proven and tested method in many areas of application and with numerous dedicated (open-source as well as commercial) simulation environments ${ }^{31}$.

\footnotetext{
${ }^{31}$ See http://en.wikipedia.org/wiki/List_of_discrete_event_simulation_software for a listing (last accessed: March 26, 2013).
} 
The DEVS formalization is constructed using set-theory, which allows to represent the (within the informal description) mentioned sets of states and rules. Through its hierarchical and modular specification it allows for an iterative decomposition into collections of (modular) sub-model-hierarchies, which, in turn, can be used for a bottom-up model implementation (while still remaining a topdown design). It distinguished between two classes of models - atomic and coupled models. Formalization of both is based upon (Fishwick, 2007a, p. 6-3 ff.), who himself boiled down two of Zeiglers books ${ }^{32}$.

\section{Atomic Models}

As the name indicates, atomic models (AM) are the smallest units and cannot be further decomposed. They are formally defined as 7-tuple of form:

$$
\mathrm{AM}=\left\langle X, S, Y, \delta_{\text {ext }}, \delta_{\text {int }}, \lambda, \text { ta }\right\rangle
$$

where

$X \ldots$ set of input events,

$S \ldots$ set of sequential states,

$Y \ldots$ set of output events,

ta: $S \rightarrow \mathbb{R}_{\geq 0} \ldots$ time advance function,

$\delta_{\text {int }}: Q \rightarrow Q \ldots$ internal transition function,

where $Q=S \times \mathbb{R}_{\geq 0}=\{(s, e) \mid s \in S$ and $0 \leq e \leq \operatorname{ta}(s)\} \ldots$ states set,

$\lambda: Q \rightarrow Y \ldots$ output function,

$\delta_{\text {ext }}: Q \times X \rightarrow Q \ldots$ external transition function.

Thus the atomic model is defined via three sets and four characteristic functions. In line with external and internal triggers/events, there are internal and external transition functions. This formalization differs a bit from Zeiglers (from 2000), as it specifies the internal transition as $\delta_{\text {int }}: Q \rightarrow Q$ instead of $\delta_{\text {int }}: S \rightarrow S$, i.e. the state changes from $q \in Q$ to $q^{\prime} \in Q$. As this notation of $q=(s, e) \in Q$ represents a state and the associated elapsed time at that state, it allows to explicitly specify a condition for when an internal transition occurred. Similar reasoning applies to the definition of the external transition function and the output function.

\section{Coupled Models}

Again, the name gives away the idea behind it: coupled models $(\mathrm{CM})$ are compositions of either atomic or coupled models. Thus it is possible to create hierarchic constructions and subsequently complex models. Formally CM are defined as 8-tuple of the form

$$
\mathrm{CM}=\left\langle X, Y, D,\left\{M_{i} \mid i \in D\right\}, \text { EIC, EOC, IC, Select }\right\rangle
$$

where

$X \ldots$ set of input events,

$Y \ldots$ set of output events,

\footnotetext{
${ }^{32}$ The above mentioned "Multifacetted Modelling and Discrete Event Simulation" (1984, Academic Press) and the $2^{\text {nd }}$ edition of "Theory of Modelling and Simulation" (2000, Academic Press).
} 


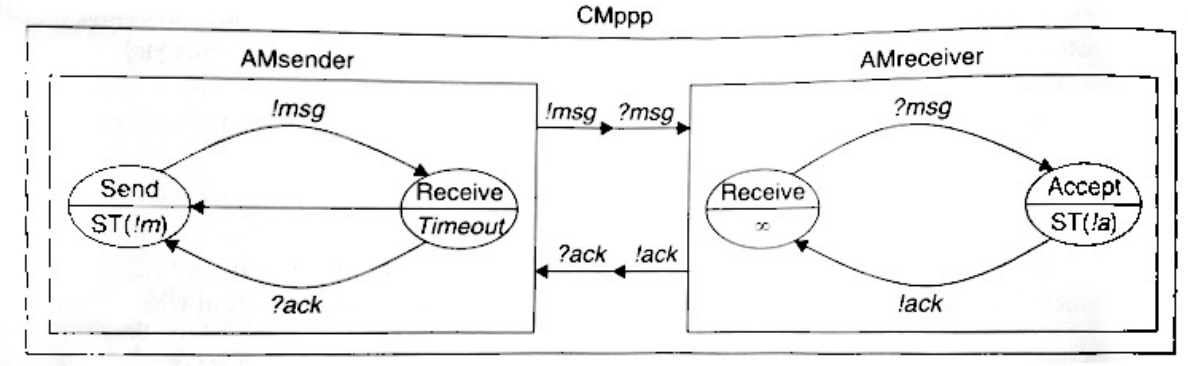

Figure 3.28: Coupled DEVS model consisting of two atomic models (sender and receiver; source: Fishwick (2007a, p. 6-2 ff.)).

$D$... set of component names,

$M_{i} \ldots$ a DEVS component - either an atomic or coupled model,

EIC $\subseteq X \times \cup_{i \in D} X_{i} \ldots$ external input coupling relation,

EOC $\subseteq \cup_{i \in D} Y_{i} \times Y \ldots$ external output coupling relation,

IC $\subseteq \cup_{i \in D} Y_{i} \times \cup_{j \in D} X_{j} \ldots$ internal coupling relation,

Select: $2^{D}-\emptyset \rightarrow D \ldots$ tie-breaking selector in case of simultaneous events.

The coupled model consists of four sets, of which the set $M_{i}$ of components is forming a coupled model and four functions. The functions (coupling relations) are set's of ordered pairs of events $(e 1, e 2)$, which instantaneously transmit all information from event $e 1$ to $e 2$. EIC specify how output events of a component (of the $\mathrm{CM}$ ) are connected to the output events of the CM. The same is the case for EOC and output events while the IC defines intra-CM coupling of internal output to internal input events.

\section{DEVS Example}

To illustrate the DEVS formalization following example from Fishwick (2007a, p. 6-2 ff.) (displayed in Fig. 3.28) shall be used. A ping-pong messaging protocol consisting of two coupled atomic models (a sender and a receiver). The sender sends a message to the receiver, which, if it receives it, acknowledges this. In case that this acknowledgment reaches the sender, another message is sent. In case that anything goes wrong, the sender is experiencing a timeout while in state "Receive" and proceeds to sending another message (potentially the same as before) to the Sender.

Formally the sender's atomic model can be described as

$$
\text { AMsender }=\left\langle X, S, Y, \delta_{\text {ext }}, \delta_{\text {int }}, \lambda, \text { ta }\right\rangle
$$


where

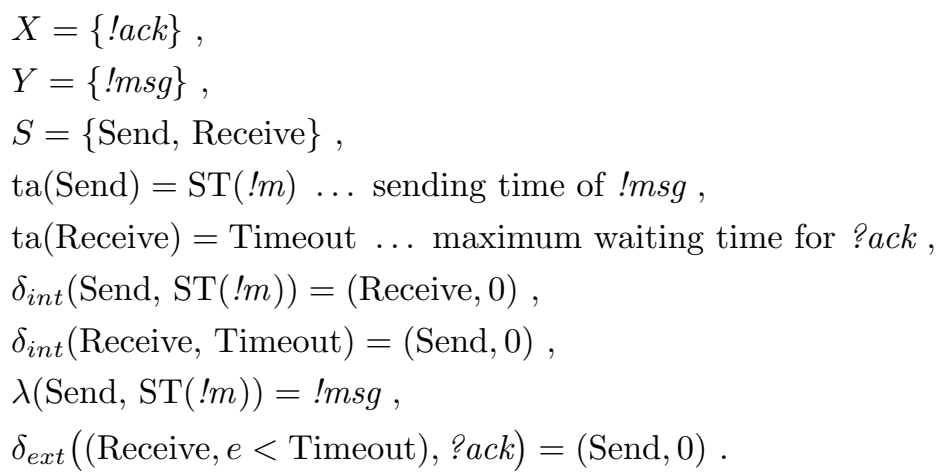

Each of the states in the atomic model has a specified sojourn time, defined by the time advance function $\mathrm{ta}()$. These are ST(!m) and Timeout in the sender's and ST(! $a)$ as well as $\infty$ in the receiver's model. The internal transition $\delta_{\text {int }}($ Send, $\mathrm{ST}(! m))=($ Receive, 0$)$ causes the sender to change from state "Send" to the beginning (which is 0 ) of "Receive", after the sojourn time ST $(! m)$ is elapsed. As mentioned before, this atomic model has a built in timeout $\left(\delta_{\text {int }}(\right.$ Receive, Timeout $)=($ Send, 0$\left.)\right)$ causing a transition from "Receive" to "Send" in case that no external event (arrival of ?ack) triggers it.

The receiver's atomic model is defined analogously. But, as it has no timeout that would serve as internal trigger for a state change, the time advance function ta(Receive) is set to $\infty$; the model does not know for how long it has to stay in the "Receive" state.

$$
\text { AMreceiver }=\left\langle X, S, Y, \delta_{e x t}, \delta_{i n t}, \lambda, \text { ta }\right\rangle
$$

where

$$
\begin{aligned}
& X=\{? m s g\}, Y=\{! a c k\}, S=\{\text { Accept }, \text { Receive }\} \\
& \operatorname{ta}(\text { Accept })=\mathrm{ST}(! a), \operatorname{ta}(\text { Receive })=\infty \\
& \delta_{\text {int }}(\text { Accept }, \mathrm{ST}(! a))=(\text { Receive }, 0) \\
& \delta_{\text {int }}(\text { Receive }, \infty)=(\text { Send }, 0) \\
& \lambda(\text { Accept }, \mathrm{ST}(! a))=! a c k \\
& \delta_{\text {ext }}((\text { Receive }, \infty, ? m s g)=(\text { Accept }, 0)
\end{aligned}
$$

Finally these two atomic models are combined to form the coupled model CMppp, which defines the interactions between the sub-models (in this case both are of atomic type, but the sub-models could also be of type combined themselves), as well as the connection of the outside (in- and output).

$$
\mathrm{CMppp}=\left\langle X, Y,\left\{M_{i}\right\}, \text { EIC, EOC, IC, Select }\right\rangle
$$


where

$$
\begin{aligned}
& X=Y=\emptyset, \\
& \left\{M_{i}\right\}=\{\text { AMsender, AMreceiver }\} \text {, } \\
& \mathrm{EIC}=\mathrm{EOC}=\emptyset \ldots \text { no interaction with environment, } \\
& \mathrm{IC}=\{\text { (AMsender.!msg, AMreceiver.?msg), } \\
& \text { (AMreceiver.!ack, AMsender.?ack) \}, } \\
& \text { Select }(\{\text { AMsender, AMreceiver }\})=\text { AMsender . }
\end{aligned}
$$

As visible by $X, Y$, EIC and EOC being empty sets $(\emptyset)$, there is no communication or interaction with the outside of the coupled model. The IC set defines the coupling between the sub-models. In this case this consists of the two pairs for sending (!) and receiving (?) of messages $(m s g)$ and acknowledgements (ack), respectively. The "Select" function finally defines that in case of concurrent events (change of state) the sender is being prioritized.

\subsubsection{Cellular Automata (CA)}

Cellular automata are, as the name indicates, based on cells. These cells are arranged as a (regular) lattice, assume discrete states and are updated (synchronously) in discrete time steps according to a uniform set of rules. These update rules define the new state of all cells and depend on the states of the respective cell's neighborhoods (i.e. its neighboring cells). Despite their apparent simplicity - which makes it easy to implement CA on computers - they exhibit a complex, unpredictable behavior - which makes them usable for (bottom-up) simulation of complex problems.

\section{Development of CA}

The concept of cellular automata evolved in the late 1940-ies around a group of people working at the Manhattan-Project, most notably John von Neumann ${ }^{33}$ and Stanisław Ulam ${ }^{34}$. The idea of CA is very similar to that of the Turingmachine, with the exception that all cells are updated simultaneously.

The concept of cellular automata can be used for an arbitrary number of dimensions, although one-, two- and three-dimensional ones are the most common ones. In his Book "A new kind of science" Wolfram (2002) analyzes and classifies one-dimensional CA on 1200 pages and concludes, that the whole universe is a cellular automaton and that CA provide a complementary kind of science. Although his work doubtlessly presents a great contribution to the exploration of (one-dimensional, 1D) CA, he has been criticized for these conclusions especially the latter one. An example for the use of 1D CA is the simulation of simplified traffic flow, where the cells represents segments of a street and their possible states are "empty" or "speed of vehicle on segment".

In two dimensions (2D) the behavior of CA naturally becomes even more complex than in 1D. One of the most impressive examples for a 2D CA is the one used by Thomas C. Schelling, winner of the Nobel Memorial Prize in Economic Sciences in 2005. In his model, described in (Schelling, 2006, p. $147 \mathrm{ff}$.), he

\footnotetext{
${ }^{33}$ Born as János Neumann Margittai in Budapest

${ }^{34}$ Born in Lwiw, and also known as Stanley Ulam after changing his Polish name.
} 
analyzes individuals' preferences on choosing a neighborhood to live in. His "segregation model" exhibits quite contra-intuitive behavior, as the individuals' preference " $50 \%$ of my neighbors should be of same race as I am" does not lead to a mixed population but to one living in segregated neighborhoods. An application example for three dimensional CA is simulation of tumor growth, as described in Kansal et al. (2000).

Besides expanding into more dimensions, there have also been extensions of the CA definition. The search for a possibility to simulate fluid flows led to development of lattice-gas cellular automata (LGCA) and the lattice Boltzman method (LCB), which are "promising methods for the numerical solution of (nonlinear) partial different equations" (Wolf-Gladrow, 2000, p. 2). They introduce stochastic behavior and expand the definition of CA as cells don't hold a single state, but up to six particles that move around within the CA. In his work Wolf-Gladrow extensively describes the development of the methods, including dead ends such as the first proposed LGCA (the HPP ${ }^{35}$ ), which, despite conservation of mass and momentum, does not lead to the Navier-Stokes equation - a necessity for modeling of the behavior of gases or liquids.

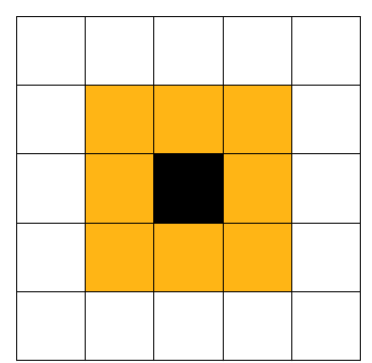

(a) Moore neighborhood with radius one.

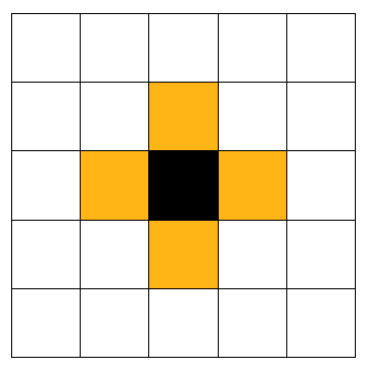

(c) Von Neumann neighborhood with radius one.

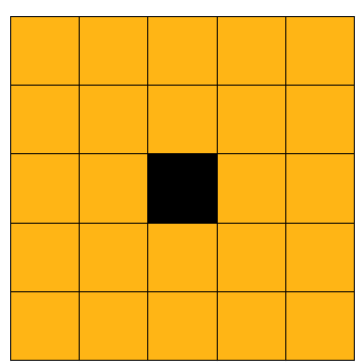

(b) Moore neighborhood with radius two.

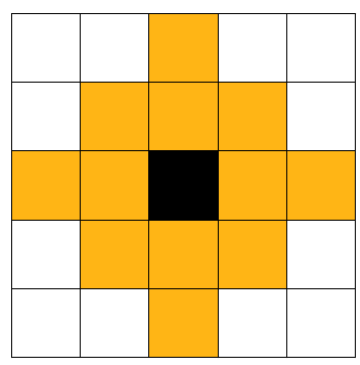

(d) Von Neumann neighborhood with radius two.

Figure 3.29: Moore (top) and von Neumann (bottom) neighborhoods with radius 1 and 2 .

A very well known and simple example for a (2D) CA is Conway's Game of Life. It is based on two cell states (dead and alive) and only four rules:

1. A live cell with less than two live neighbors dies (solitude).

2. A live cell with two or three live neighbors stays alive.

\footnotetext{
${ }^{35}$ Named after Hardy, de Pazzis and Pomeau
} 
3. A live cell with more than three neighbors dies (overcrowding).

4. A dead cell with exactly three live neighbors becomes alive.

And the neighborhood used is the Moore neighborhood with radius 1 (see Fig. 3.29 which shows the two most common neighborhood types for radius one and two).

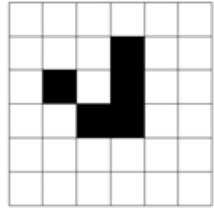

$t=0$

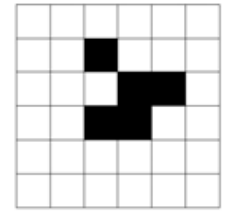

$t=1$

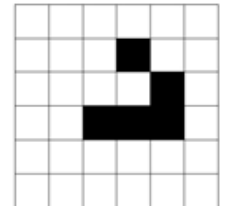

$t=2$

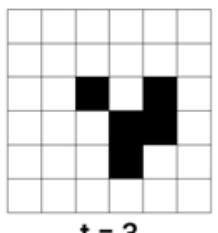

$t=3$

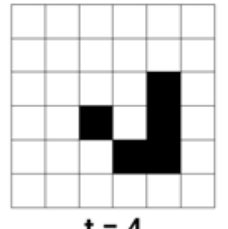

$t=4$

Figure 3.30: One period of the self-propagating structure "Glider" from Conway's Game of Life.

John Conway, the developer of the game ${ }^{36}$ awarded a prize to the first person to find an infinitely growing pattern. This prize money created a frenzy around the game which lead to a very high popularity and partially explains its nowadays cult-status among computer enthusiasts ${ }^{37}$. The prize itself went to a team at the MIT around Bill Gosper that found a periodic pattern (periodicity 30 ) that is producing "Gliders" (a self-propagating structure with periodicity 5 , see Fig. 3.30) and sending them into (theoretical ${ }^{38}$ ) infinity. This pattern has come to be known as Gosper glider gun.

\section{Mathematical Formalization of a CA}

Following formalization of cellular automata is based upon the definition of the research group Mathematical Modelling and Simulation (MMS) at TU Vienna, elaborated by Schneckenreither et al. (2013). A cellular automaton consists of the elements:

- Cell

- The set of all cells will be denoted $M$. Consequently a cell is a unique (A set contains by definition unique elements.) element $m \in M$. As there is no limit to the number of cells within an automaton, these can be finite or infinite depending on the number of cells.

- Lattice

- Index set:

A subset $I$ of $\mathbb{Z}^{d}$ is called connected if for each two elements $a, b, \in I$ there exists a series of elements $\left(z_{\alpha}\right)_{\alpha \in \mathbb{N}} \subset I$ for which $\left\|z_{\alpha}-z_{\alpha+1}\right\|=1 \forall \alpha \in \mathbb{N}$ and for which $a, b \in\left(z_{\alpha}\right)_{\alpha \in \mathbb{N}}$. A connected subset $I \subseteq \mathbb{Z}^{d}, d \in \mathbb{N} \backslash\{0\}$ is called an index set.

\footnotetext{
${ }^{36}$ The term game is a bit misleading, as it works without any user-interaction except for specification of the initial state.

${ }^{37}$ The Game of Life is still the target of scientific research and has a very active community surrounding it. Its Wiki can be found at http://www.conwaylife.com/wiki/Main_Page .

${ }^{38}$ As computer memory is limited, so is the lattice of the Game of Life. Nevertheless, theoretically these Gliders would propagate into infinity of space.
} 
- Index mapping:

If $M$ is a set of cells, we call $M$ indexed or regularly arranged if there exists a bijective mapping $\mathcal{I}: M \rightarrow I: m \mapsto \mathcal{I}(m)=i$ between $M$ and an index set $I$.

We call $\mathcal{I}$ an index mapping and also use $\mathcal{I}$ for mapping tuples of cells onto tuples of indices

$$
\mathcal{I}: M^{k} \rightarrow I^{k}:\left(m_{1}, \ldots, m_{k}\right) \mapsto\left(\mathcal{I}\left(m_{1}\right), \ldots, \mathcal{I}\left(m_{k}\right)\right)=:\left(i_{1}, \ldots, i_{k}\right)
$$

where $k \in \mathbb{N} \backslash\{0\}$.

$\left(m_{\mathcal{I}(m)}\right)_{m \in M}$ is the natural indexing for a set of indexed cells.

Since by now all cells are "arranged" or indexed using index set $I \subseteq \mathbb{Z}^{d}$, we call $d$ the dimension of the cellular automaton.

- Neighborhood

- Relative and absolute index tuples:

Let $k \in \mathbb{N} \backslash\{0\}$.

A tuple $J:=\left(j_{1}, \ldots, j_{k}\right) \in\left(\mathbb{Z}^{d}\right)^{k}$ where $j_{\alpha} \neq j_{\beta}$ is called a relative index tuple and for $i \in \mathbb{Z}^{d}$ the addition respectively subtraction

$J \pm i:=\left(j_{1} \pm i, \ldots, j_{k} \pm i\right)$ is well-defined.

Given a relative index tuple $J$ we define the index translation $\mathcal{T}_{J}$ of an index $i$ by

$$
\mathcal{T}_{J}: I \rightarrow\left(\mathbb{Z}^{d}\right)^{k}: i \mapsto i+J
$$

and call the result an absolute index tuple.

Note that $i+J$ is not necessarily a subset of $I$.

- Indexed neighborhood:

For a cell $m_{i}$ from an indexed set of cells with index set $I$ and a relative index tuple $J$ we use the resulting absolute index tuple $\mathcal{T}_{J}(i)=\left(i_{1}, \ldots, i_{k}\right) \in\left(\mathbb{Z}^{d}\right)^{k}$, to define the neighborhood of $m_{i}$ as

$$
N_{m_{i}, J}:=\left(n_{1}, \ldots, n_{k}\right) \in(M \cup\{\emptyset\})^{k}
$$

where

$$
n_{\alpha}:=\left\{\begin{array}{ll}
m_{i_{\alpha}}=\mathcal{I}^{-1}\left(i_{\alpha}\right) & i_{\alpha} \in I \\
\emptyset & i_{\alpha} \notin I
\end{array} \quad \alpha \in\{1, \ldots, k\}\right.
$$

Furthermore we call $k$ the neighborhood dimension.

The nonexistent cell $\emptyset$ is required in order to maintain the original tuple structure of the neighborhood and to be able to indicate that indices which are outside the index set do not refer to a cell.

- Characteristics:

A cell lies in its own neighborhood (reflexive) if and only if $0 \in \mathbb{Z}^{d}$ is part of the relative index tuple. An index tuple respectively neighborhood is neither necessarily symmetric, bidirectional nor local.

- Neighborhood mapping:

For an indexed set of cells $\left(M, I, \mathcal{I}, \mathcal{I}^{-1}\right)$ and an index translation $\mathcal{T}$ the neighborhood mapping is defined as

$$
\begin{aligned}
\mathcal{N}:=\mathcal{I}^{-1} \circ \mathcal{T} \circ \mathcal{I}: M & \rightarrow I \rightarrow \mathbb{Z}^{k} \rightarrow(M \cup\{\emptyset\})^{k}: \\
m_{i} & \mapsto i \mapsto\left(i_{1}, \ldots, i_{k}\right) \mapsto\left(n_{1}, \ldots, n_{k}\right) .
\end{aligned}
$$


- Border

- Special cells:

A border cell is a cell, which is located at the boundary of the lattice. If the absolute index tuple of a cell $m_{i}$ does not lie completely within the index set $\left(\mathcal{T}_{J}(i) \nsubseteq I\right)$, we talk of (a cell with) a degraded neighborhood.

- Extended geometry:

In order to manipulate the geometry of a lattice (e.g. periodic boundary conditions) we modify the index translation:

Given a relative index tuple $J$, the generalized index translation is defined by $\mathcal{T}_{J}: I \rightarrow I^{k}: i \mapsto\left(i_{1}, \ldots, i_{k}\right)$ where

$$
i_{\alpha}:=\left\{\begin{array}{ll}
i+j_{\alpha} & i+j_{\alpha} \in I \\
\tau\left(i+j_{\alpha}\right) & i+j_{\alpha} \notin I
\end{array} \quad \alpha \in\{1, \ldots, k\} .\right.
$$

and $\tau: \mathbb{Z}^{d} \backslash I \rightarrow I$.

- State

- State mapping:

Let $k \in \mathbb{N} \backslash\{0\}$ be the neighborhood dimension.

There exists a (temporary) state mapping form the set of all cells $M$ to the set of all possible states $\mathbb{S}$, which assigns a state to each cell: $\mathcal{S}: M \rightarrow \mathbb{S}: m \mapsto \mathcal{S}(m)=s$. We also use $\mathcal{S}$ as

$$
\mathcal{S}:(M \cup\{\emptyset\})^{k} \rightarrow(\mathbb{S} \cup\{\emptyset\})^{k}:\left(m_{1}, \ldots, m_{k}\right) \mapsto\left(s_{1}, \ldots, s_{k}\right)
$$

where

$$
s_{\alpha}:=\left\{\begin{array}{ll}
\mathcal{S}\left(m_{\alpha}\right) & m_{\alpha} \in M \\
\emptyset & m_{\alpha} \notin M \Longleftrightarrow m_{\alpha}=\emptyset
\end{array} \quad \alpha \in\{1, \ldots, k\} .\right.
$$

The nonexistent state $\emptyset$ is required to maintain the tuple structure and to indicate a degraded neighborhood.

- State-space:

The set of all possible states $\mathbb{S}$ may contain a finite or infinite number of states.

A "state-space" (coll.) can be a vector space, a ring or any other algebraic structure.

By introducing a partitioning on the set of all possible states, different cell types can be distinguished.

A non-trivial cellular automaton features more than one different element in $\mathbb{S}$.

- Update

- Preliminary:

An update rule (update rule set) can be the explicit definition of a mapping but also a (continuous) function or a combination of functions. 
Since degraded neighborhoods contain non-existent cells respectively states $(\emptyset)$, an update rule must react on a degraded neighborhood and implement (arbitrary) boundary conditions.

Update rules never define the geometry of the lattice! Further, stochastic update rules are excluded from the basic definition, as the necessary introduction of a probability space would be an extension to the formal definition.

An update rule must be defined for every possible neighborhood configuration. Otherwise the automaton would exhibit undefined behavior.

Update rules have to be compatible with the index set: All occurring degradation of neighborhoods has to be taken into account.

Update rules must be self-contained since all possible state-configurations (except for the initial condition) arise from the update rule.

- Update rule:

Let $k \in \mathbb{N} \backslash\{0\}$.

An update rule is a mapping

$$
\mathcal{F}:(\mathbb{S} \cup\{\emptyset\})^{k} \rightarrow \mathbb{S}:\left(s_{1}, \ldots, s_{k}\right) \mapsto s
$$

To calculate a new state for a cell:

$$
\begin{aligned}
\mathcal{F} \circ \mathcal{S} \circ \mathcal{N}: M & \rightarrow(M \cup\{\emptyset\})^{k} \rightarrow(\mathbb{S} \cup\{\emptyset\})^{k} \rightarrow \mathbb{S}: \\
m & \mapsto\left(m_{1}, \ldots, m_{k}\right) \mapsto\left(s_{1}, \ldots, s_{k}\right) \mapsto s
\end{aligned}
$$

- Global state

- The state of all cells is accumulated in the temporary state mapping $\mathcal{S}$ which can be identified with an element of $\mathfrak{S}:=\mathbb{S}^{M} . \mathcal{S}$ is then also called the (temporary) global state.

Given a neighborhood mapping $\mathcal{N}$, a temporary mapping $\mathcal{S}$ and an update rule $\mathcal{F}$ we define the local evolution operator

$$
\begin{aligned}
\tilde{\mathcal{S}}:= & \mathcal{F} \circ \mathcal{S} \circ \mathcal{N}: \\
& M \rightarrow(M \cup\{\emptyset\})^{k} \rightarrow(\mathbb{S} \cup\{\emptyset\})^{k} \rightarrow \mathbb{S}: \\
& m \mapsto\left(m_{1}, \ldots, m_{k}\right) \mapsto\left(s_{1}, \ldots, s_{k}\right) \mapsto s .
\end{aligned}
$$

Thus the local evolution operator is a state mapping and a global state.

- Iteration

A (global) evolution operator is a mapping

$$
\mathcal{E}: \mathfrak{S} \rightarrow \mathfrak{S}: \mathcal{S} \mapsto \tilde{\mathcal{S}}:=\mathcal{F} \circ \mathcal{S} \circ \mathcal{N}
$$

An iterative process can be obtained by defining

$$
\mathcal{S}_{t+1}:=\mathcal{E}\left(\mathcal{S}_{t}\right)=\mathcal{F} \circ \mathcal{S}_{t} \circ \mathcal{N}
$$

where $t \in \mathbb{N}$.

For iteration, an initial state or initial condition $\mathcal{S}_{0}$ must be given. It is necessary that the initial condition is compatible with the update rules. 
With these elements defined it is possible to formally compose a cellular automaton. According to the above, a CA comprises of ...

1. an index set of cells,

2. an initial state mapping $\mathcal{S}_{0}$,

3. a relative index tuple $\mathcal{J}$,

4. a generalized index translation (if the geometry is to be "manipulated"),

5. an update rule $\mathcal{F}$, and

6. the iterative application of an evolution operator.

\subsubsection{Agent-Based Methods}

Autonomous agents and as a consequence systems composed of such, i.e. multiagent systems (MAS), are a relatively new subject. Thus they struggle with problems common to early development stages of scientific disciplines, most importantly the lacking of a universal definition. In addition the subject is an inter-disciplinary one, which adds to the complexity and gives it a bit of a chaotic appearance. The origins of agent-based (AB) methods may be traced back to complex adaptive systems, artificial intelligence (AI) and distributed artificial intelligence (DAI). Similar to the situation of these fields in the 1980s, and despite the mentioned issues, $\mathrm{AB}$ systems (ABS) are receiving very much attention - with the immanent danger of becoming over-hyped. Nevertheless ABS pose a very valuable and worthwhile approach for many problems.

The AB systems are defined its smallest units - the agents. Accordingly ABS are classified as bottom-up approach. As simulation of large systems with large numbers of (interacting) agents (i.e. MAS) requires sufficient computing resources, thus the increase of computing power over the last decade is also a reason for their success-story. Another one is their (potential) use in social sciences, which has even been described as "the breakthrough in computational modelling in the social sciences" by Gilbert and Terna (2000, p. 60). The reason for this enthusiasm is that the $\mathrm{AB}$ approach allows modeling of virtually any (complex) behavior of the entities within a system. While cellular automata (CA) also facilitate this idea to some degree, agents take it a whole lot farther. Although, as previously mentioned, there is no universally accepted definition of an (autonomous) agent yet. Nevertheless, many definitions have a common core, or use similar terminology.

\section{Definition of Agent-based Systems}

Following definition ${ }^{39}$ of Jennings (2000, p. 280) is arguably a solid basis.

"An agent is an encapsulated computer system that is situated in some environment and that is capable of flexible, autonomous action in that environment in order to meet its design objectives."

\footnotetext{
${ }^{39}$ Besides this definition in "On agent-based software engineering" (2000) he worked out his ideas in several previous publications (e.g. Jennings et al. (1998)) which are also often cited in respective literature.
} 
According to it agents are characterized as:

- encapsulated,

- situated in an environment,

- flexible,

- autonomous and

- goal-oriented.

A full ten years later Macal and North (2010, p. 135) still see that "there is no universal agreement ... on the precise definition of an agent beyond the essential property of autonomy" and also acknowledge Jennings' above definition as "a computer science definition ... [emphasizing] the essential characteristic of autonomous behaviour." They further propose a definition "from a practical modeling point of view" which differentiates between an agent's essential and other useful characteristics. The former state that an agent has or is

- self-contained,

- autonomous behavior,

- states (that change over time),

- social,

while the latter characteristics are

- adaptiveness,

- goal-orientation and

- heterogeneity (of agents).

Since Jennings explained encapsulated as "clearly identifiable ... with welldefined boundaries and interfaces", and autonomous as "they have control both over their internal state and over their own behaviour", the definitions differ only marginally. In this sense Jennings' "encapsulated" can be put on a level with "self-contained" from Macal and North while "autonomous" includes both "autonomous behavior" and "varying states". The definition(s) also explain the popularity of ABS among social sciences: with the possibility do define heterogeneous agents with a given behavior that interact autonomously with(in) their environment, potentially even with an evolving /adaptive behavior, they are predestined for complex interacting systems - like the real world.

Modeling of complex systems usually requires multiple agents, thus multiagent systems (MAS). Besides containing multiple agents the characteristics of these systems is (see Jennings et al., 1998, p. 17) that:

- each agent only has incomplete information (i.e. a limited or local point of view),

- the system has no global control,

- data is decentralized and 
- computation is asynchronous.

Implementation of MAS is ideally done using object-oriented (OO) programming, as it logically supports modeling of the agents' autonomous (and computationally asynchronous) nature. Object-oriented programming environments not only allow to create multiple independent instances of an object (i.e. agent) but also to - via inheritance - create a heterogeneous population of related objects.

While agents' behavior can be defined by plain code, it is often done using state diagrams or statecharts (e.g. UML state diagrams). As shown in Fig. 3.31, a statechart is easily read and understood, which facilitates interdisciplinary communication and cooperation. Even if the precise conditions for state changes are not obvious from the diagram alone, it is possible to verify the general agent behavior. Another advantage, besides easy optical verification, of state diagrams is their easy modification, which allows to quickly adapt agent behavior. This is also shown in Fig. 3.31, where the statechart is modified (from left to right) in order to add "field work" to the agent's behavior.
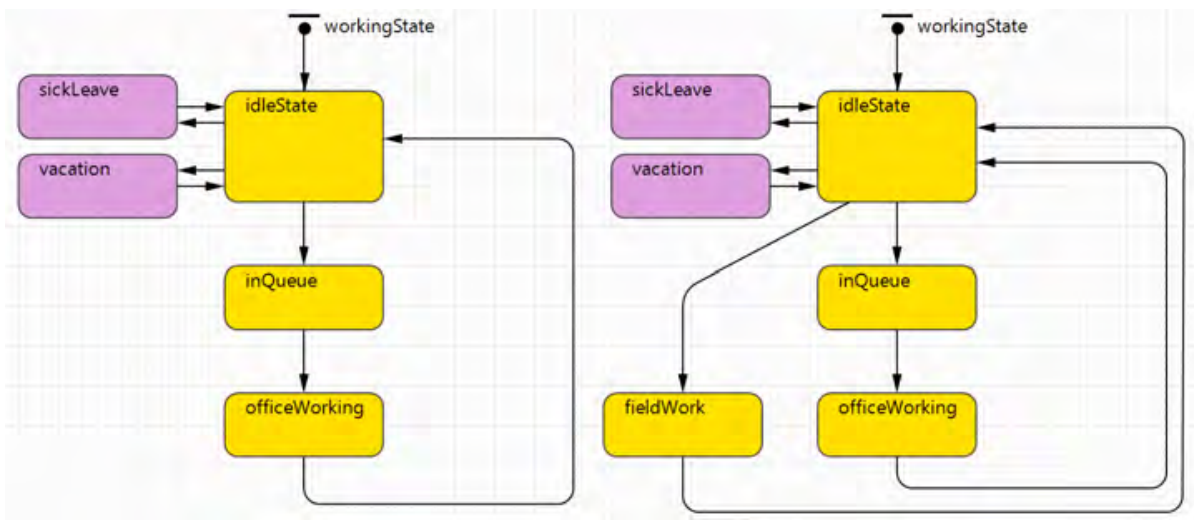

Figure 3.31: Example of an AnyLogic statechart depicting behavior of an office employee (left) and extension of statechart (right) to reflect behavior of employee with office and field work.

\section{Hybrid coupling of Agent-based features}

Due to agents' autonomous and asynchronous nature, implementation of MAS poses a great challenge in terms of conflicting or simultaneous events. An event affecting a single agent can cause a chain reaction of events that leads to a simulation outcome that drastically differs from the development if a concurring event would have been executed in the first place. For this reason accurate simulation requires some sort of state event finder / event scheduling. In addition to the processing of simultaneous events, stochastic elements within agent behavior also influence simulation outcome. The Monte Carlo method is often applied to overcome these problems (or at least the stochastic influence) and to generate more accurate results.

Taking a look back at cellular automata (CA) and discrete event simulation (DES), one can see that these methods do not have the problem of concurrent events (cellular automata) or are explicitly designed with the problem in mind 
(DEVS). On the other hand these methods are confined to a handful of states (CA) or to processing of only a few different servers and products ${ }^{40}$. This contrast, and the fact that all three methods are bottom-up approaches, makes it interesting to develop hybrid models by combining features of CA or DES respectively with the $\mathrm{AB}$ approach. This is supported by other overlapping characteristics, such as limited/local information and spatial embedding ( $\mathrm{AB}$ and $\mathrm{CA}$ ) or discrete events causing state changes (AB and DES). With a hybrid model it is possible to add individual memory or information to otherwise identical copies of an object and still have the advantage of a CA's simplicity or of the well-defined structure of the discrete-event systems specification.

Finally, it is necessary to note that the relationship between agents and simulation is a multifaceted one (see Fishwick, 2007a, p. 8-10). According to Fishwick there are two aspects in modeling of and with agents. On the one hand agents are used as a metaphor for designing software systems and for the modeling of dynamic systems which are than to be evaluated by simulation. On the other hand simulation is used to evaluate agents (e.g. for analysis of cooperation or problem solving strategies).

\footnotetext{
${ }^{40}$ Of course the interpretation of server and product can be a different one, such as doctor and patient.
} 


\title{
Chapter 4
}

\section{Application and Case Study}

\begin{abstract}
After filling the toolbox with the tool set elaborated in the preceding chapter, this one focuses on its application. For this the MoreSpace project (introduced in the consecutive section) will serve as case study. In section 4.1 the respective system will be analyzed and deployment of the model prepared. Due to the "Problems Encountered" (section 4.1.3) a modification of the project becomes necessary (section 4.2) including a change of the intended deployment as well as development of alternative support tools (based on information visualization). The architecture of the hybrid simulation-model itself will be covered briefly in 4.2.1. The lessons learned and experiences gained in the course of the project finally motivate a digression into space management of office buildings, including development of a simulation model (section 4.3).
\end{abstract}

\section{Genesis of the MoreSpace-Project}

In 2004, after receiving increased autonomy through the UG 2002 (see section 1.2.4), talks regarding a potential emigration of the Vienna University of Technology (TU Vienna ${ }^{1}$ ) were initiated as the province of Lower Austria (Niederösterreich) offered to subsidize the migration of two faculties (electrical engineering and chemistry) to Tulln (a city $45 \mathrm{~km}$ NW of Vienna) with $€ 300$ million. According to Krause (2005), the city of Vienna responded at the last moment (the deal should have been officially proclaimed on January $25^{\text {th }} 2005$ ), by offering eight alternative locations within the city boundaries to the TU Vienna on January $21^{s t}$.

What followed was a long and intense discussion which lead to the formation of a group surrounding professor Hierzegger (see Skalicky, 2007, p. 3), which was in charge of assessing all options and possibilities. By 2006 the group had narrowed down the possibilities to two. The first one was to build the university from scratch at the "Airport Aspern", the site of Vienna's former airport ${ }^{2}$, located in its $22^{\text {nd }}$ district. The second was to maintain the innercity location and restructure accordingly. While rector Skalicky favored the

\footnotetext{
${ }^{1}$ Despite its English name being Vienna University of Technology the university chose "TU Vienna" as its short form, instead of the expected "Vienna UT" (VUT). The reason for this is not known to the author. Rumor has it that officials were afraid of the similarities of "VUT" to "Fut" in German pronunciation - with the latter being vulgar slang for vagina.

${ }^{2}$ Nowadays it is (one of) Vienna's largest real estate development areas. Construction began in 2010 .
} 
latter option, staff and affiliates opposed it, so that in June 2006 the rectorate (in unison) decided to pursue the option favored by the staff. This decision was supported by the Universitätsrat ${ }^{3}$. Thus the option for the university to remain at its inner city location and renovate, reorganize the buildings to achieve an infrastructure level comparable to that of a newly built campus at a new location, was selected to be pursued. The project, including financing of it, was accepted by the Austrian government and the city of Vienna, and subsequently named "Univercity 2015" in fall 2005. The name points at its intended finishing deadline in 2015, when the university is going to celebrate its $200^{\text {th }}$ anniversary.

As mentioned the concept included renovation of all buildings and reorganization of the eight faculties, such that each two faculties were assigned one of the four main groups of buildings in the city center - all of it parallel to regular day-to-day operations. The university's former rector Peter Skalicky (1991-2011) thus compared the process with the ambition of changing a car's tire during full speed.

With construction work also taking place during regular teaching periods it was necessary to compensate for closed/blocked auditoria. Finding alternatives for large auditoria in order to accommodate lectures and exams in an adequate manner is especially tricky. And even more so if these alternative spaces are to be close to campus in order to minimize commute. In addition these spaces have to be rented and thus payed for. While the project budget initially (presumably) covered all the necessary costs, the respective ministry "changed the rules during the game", as current rector Sabine Seidler put it (see Dzugan, $2012)^{4}$. This not only abruptly stopped or at least strongly changed the project "Univercity 2015", but also further increased financial strain on the chronically under-financed TU Vienna ${ }^{5}$.

The financial development (see Table 4.1) alongside a steady increase of student numbers - and with it the need for additional space for teaching - caused the university to search for strategies to improve the situation. According to Prof. Wiegand "vice rector Prof. Schimak, who heard and read about my [Prof. Wiegand's - author's note] research conducted at the ETH Zürich (see Mebes et al., 2006; Wiegand et al., 2008), asked me if it would be possible to carry out such an analysis for TU Vienna".

In order to evaluate the effective need for lecture rooms and to find a strategy to reduce renting additional space in 2008 feasibility studies were conducted by the university's department for building, construction and technology (GuT, derived from its German name "Gebäude und Technik"), the working group "Mathematical Modelling and Simulation" $\left(\mathrm{MMS}^{6}\right)$, and the "Chair of Real Estate Development and Management" (RED $\left.{ }^{7}\right)$

Due to the promising nature of its outcome the three organizational units

${ }^{3}$ The Universitätsrat is one of the highest bodies of Austrian universities. Its role is comparable to that of a supervisory board of companies.

${ }^{4}$ Quote translated by the author; German original: "die Spielregeln während des Spiels geändert"

${ }^{5}$ As of May 2012 the Vienna University of Technology piled up 20 million Euro debts (see Dzugan, 2012) - which is roughly $10 \%$ of the annual budget (see 4.1 ).

${ }^{6}$ Lead by Prof. Breitenecker, part of TU Vienna's Institute for Analysis and Scientific Computing.

${ }^{7}$ Lead by Prof. Wiegand, part of TU Vienna's Institute of Urban Design and Landscape Architecture. 
Table 4.1: Total budget of TU Vienna and building related costs (including rent, repair, energy, cleaning, etc.; sources: Vienna University of Technology (2007, 2011)) in million Euro, share of building related costs, student numbers in winter semester (source: Bundesministerium für Wissenschaft und Forschung (2012, visited: May $23^{\text {rd }}$, 2012)) and available per-student budget (total divided by student number).

\begin{tabular}{cccccc} 
year & $\begin{array}{c}\text { total } \\
\text { budget }\end{array}$ & $\begin{array}{c}\text { building } \\
\text { related }\end{array}$ & $\begin{array}{c}\text { building } \\
\text { fraction }\end{array}$ & $\begin{array}{c}\text { student } \\
\text { numbers }\end{array}$ & $\begin{array}{c}\text { budget per } \\
\text { student }\end{array}$ \\
\hline 2007 & 200.4 & 53.2 & $26.5 \%$ & 22,912 & $€ 8.747$ \\
2011 & 229.5 & 67.5 & $29.4 \%$ & 29,035 & $€ 7.904$ \\
\hline
\end{tabular}

(GuT, MMS and RED), together with the dwh $\mathrm{GmbH}^{8}$, a Vienna-based company specialized in creating customized simulation services, developed the project "MoreSpace" for which they successfully applied for funding at the "Zentrum für Innovation und Technologie" (ZIT), a funding authority of the city of Vienna ${ }^{9}$. The goal of the project which started in 2010 was to create a tool that on one hand would be able to assist GuT and TU Vienna in increasing the efficiency of space utilization and to help find solutions for the reconstruction phase. On the other hand the tool should be made as generic and flexible as possible in order to be transferable to other institutions - primarily, but not limited to, universities.

Support for this procedure was found in a survey among English universities, which showed, that their utilization was at a low 20-40\%, according to HEFCE (1999). Something that seemed to be similar for TU Vienna, as far as the feasibility study hinted. It is fascinating to note, that there is hardly any explanation for this discrepancy between perceived and real utilization. Or, as put by Beyrouthy et al. (2010, p. 364), who find it "alarming", that "there is little consensus as to whether such low utilisation rates are an inevitable result of the constraints within space planning process, or (more likely) that they can be improved by better (long-term) space planning and better (short-term) space management".

Nevertheless, when aiming at improvement of space management in educational facilities, one has to bear in mind, that space planning is something (very) different from timetabling, as the former involves multiple time-scales and also "because of the need to plan resource capacities rather than taking them as fixed" (also Beyrouthy et al., 2010, p. 365) - which is also explained and described in section 2.2.3.

\subsection{System Analysis and Pre-Deployment}

Starting out with development of the project goals, the units of measurement necessary are defined and formalized in section 4.1.1. With these in mind the target system (TU Vienna) is thoroughly analyzed (section 4.1.2). In the course of this process several problems are encountered (section 4.1.3) — which will force a modification of the project (described in section 4.2).

\footnotetext{
${ }^{8}$ Website: http://www.dwh.at/

${ }^{9}$ It literally translates to "center for innovation and technology".
} 


\subsubsection{Definitions and Objectives}

At the initiation of the project the most important goal was understanding the impact of closing (large) lecture rooms on daily business (i.e. teaching). Especially renovation of the "Audi Max", the largest auditorium at TU Vienna with an official maximum capacity of 643 seats $^{10}$, presented a critical moment. With the next largest auditoria having capacities of 400 or slightly more seats, it was understood, that an external replacement had to be found at least for the largest lectures/events.

Coming from this objective other wishes towards the simulation model were added. Among those were the desire to better understand and subsequently improve booking processes, analyze different room-allocation strategies, realtime allocation of lecture rooms for events (lectures, internal and external ones), testing of system limits (i.e. maximum number of students), advance testing for introduction of new curricula, to name a few.

Together with the GuT (department for building, construction and technology) this "wish list" was analyzed and assessed, which lead to identification of three objectives for the to be developed tool. These three were:

1. Testing of system limits or new curricula including testing the effect of blocked rooms

2. "Real-time" space management room-to-lecture assignment on a frequent (e.g. daily or weekly) basis

3. Retrospective system analysis that allows testing alternative management strategies

Which, taking a closer look, inherit correlations.

Scenario (3) was initially a "political" scenario in as far, that it could be used to prove validity of the tool using historical data. Based on the demand from recent semesters allocation proposals can be generated with different space management strategies (also referred to as booking strategy) and compared to the real course-room bookings. Since all data is available at the start of retrospective analysis, it can potentially generate better results than has been possible in reality. The reason for this is, that data from the past is known for the whole time interval in advance, while live data "trickles in" in the course of time. As more knowledge allows for better results (i.e. with all data available at once a better room allocation is possible than if allocation is done piece-wise, see section 1.2.3 for a detailed explanation), which poses a problem for comparability. To pay respect to this effect, "pooling" has been introduced to the simulation in order to collect and temporally group room-requests that entered the system. These pools are then processed by the simulation as (virtual) time progresses, taking into account the events from previously allocated pools.

For scenario (2) the pooling is necessary as well, although this time because actual input data is only gradually available. Thus pools are being processed along real time (instead of virtual time as in (3)). Thus the difference between (2) and (3) lies mainly in the input data — and in the applied space management

\footnotetext{
${ }^{10}$ The unofficial occupancy was regularly much higher, as stairs and balustrades were being used by the audience as well (past tense is used as the Audi Max is undergoing renovation at the moment and is thus out of order, author's note).
} 
Table 4.2: Increase of student numbers for architecture at TU Vienna, source: https://tiss.tuwien.ac.at/statistik/public_lehre - last visited: April 18 ${ }^{\text {th }}, 2013$.

\begin{tabular}{cccccccc} 
& $2010 \mathrm{~S}$ & $\mathrm{~W}$ & $2011 \mathrm{~S}$ & $\mathrm{~W}$ & $2012 \mathrm{~S}$ & $\mathrm{~W}$ & $2013 \mathrm{~S}$ \\
\hline Students & 4709 & 5316 & 5145 & 5757 & 5374 & 5957 & 6095 \\
Relative & $100 \%$ & $113 \%$ & $109 \%$ & $122 \%$ & $114 \%$ & $127 \%$ & $129 \%$ \\
\hline
\end{tabular}

strategies. While (3) can be used to test and evaluate various strategies, it is obvious that one can only pursue one strategy at a time in real life (2).

Finally scenario (1) can be interpreted as a mixture of both (2) and (3). Or as "retrospective simulation" with "future input data". Since, to the author's knowledge, future data does not exist, prediction or expectations are used to extrapolate existing data. Based on these assumptions it is then possible to test limits of the system (e.g. increase student numbers or block lecture rooms). It is also possible to examine which space management strategies are best suited to cope with certain situations. A real-world example for this is the steady increase of student numbers for architecture at TU Vienna by $29 \%$ over the past 7 semesters (see Table 4.2 for detailed figures). The zig-zag-pattern is explained by the fact that the majority of students - due to curricula design - enrolls in winter semester. With only few enrolling numbers drop in summer semester as students finish studies or drop out. This increase put extreme pressure not only on the respective faculty that had to cope with teaching and examining these students (without staff increase). The demand for auditoria especially for the very large introductory courses - rose proportionally.

\section{Definition of Terms and Units of Measurement}

As previously mentioned (see sections 2.2 and 3), results of alternatives cannot be compared if no standardized measurement exists. Similar as in the general timetabling vs. room-allocation problem (see section 2.2.3), there is also a difference between measuring the quality of a particular timetabling solution and the practical needs of real institutions. As McCollum (2007, p. 16 f.) points out, the latter require a measurement that combines "room usage, staff and student satisfaction". Measurement of the room usage can be done in a fairly straight forward way using operating figures and data from space management. Measuring satisfaction of staff and students (or of staff and clients, in a more general context) on the other hand is much harder. McCollum argues that staff satisfaction can be measured by the "extent to which teaching duties can be 'bunched' together" and thus leave large(r) windows of unscheduled time for "research and other activities". Similarly he proposes to use "the spread of events and the availability of choice" as measures of student satisfaction. With this approach he introduces a measurable component to an area dominated by soft constraints, although he acknowledges that "more work needs to be completed to understand the relationship between space usage, staff flexibility and student choice", and it thus is "essential that metrics are produced to measure the effectiveness of timetables from all perspectives".

Efforts to formalize measuring of room usage have already been undertaken, such as by Beyrouthy et al. (2007, 2009, 2010), which also serve as a base for the 
following definitions. Usually, and in their work, the time domain (of schedules and the like) is broken into timeslots of fixed, equal size that are periodically available (e.g. full hours or 50-minute slots). Here the timeslot is interpreted as the smallest (or atomic) temporal unit. With respect to the problem of space management, it is - from a practical as well as a technical perspective feasible to set the atomic temporal unit as one minute. For figurative reasons it is referred to as time slice instead of timeslot. This interpretation of 1minute slices increases the applicability of the following definitions and also reflects the peculiarities of Austrian universities, where no strict, system-wide timeslots exist. Lectures are usually scheduled to start at the quarters of an hour and usually last for multiples of 45 minutes, but exceptions from this are not uncommon.

Hereinafter $C_{i}$ denotes the capacity of room $i$ and $S_{i, t}$ the number of students within room $i$ at time slice $t$.

With this in mind, the total number of seat-hours is defined as

$$
B=\frac{1}{60} \sum_{i} \sum_{t} S_{i, t},
$$

and the maximum number of seat-hours as

$$
B_{M}=\frac{1}{60} \sum_{i} \sum_{t} C_{i},
$$

the sum over all rooms' capacities over all time slices - both standardized (division by 60) for a better handling/understanding. The latter definition $\left(B_{M}\right)$ neglects the possibility that rooms can be filled over capacity. The reason for this is the assumption, that events with an audience larger than the assigned room's capacity (a) either could not be provided with a room large enough (i.e. such a room does not exist at all or for the time necessary) or (b) are deliberately assigned too small of a room, as it is known/expected that over time student numbers decrease sufficiently to fit.

Occupancy of a room $i$ at time slice $t$ (denoted $O_{i, t}$ ) is defined as the fraction of seats used, thus

$$
O_{i, t}=\frac{S_{i, t}}{C_{i}} .
$$

Occupancy $O_{i}$ of a room $i$ is the average of its occupancies over all of the room's occupied time-slots $\left(t_{i}^{\text {occ }}\right)$.

$$
O_{i}=\frac{1}{t_{i}^{\mathrm{occ}}} \sum_{t} O_{i, t} .
$$

Frequency usage for a room $i$ is the fraction of used time-slots (i.e. such slots that have events assigned):

$$
F_{i}=\frac{t_{i}^{\mathrm{occ}}}{t_{i}} .
$$

Finally utilization (of room $i$ ) is the product of occupancy and frequency usage, 
which leads to

$$
\begin{aligned}
U_{i}=F_{i} \cdot O_{i} & =\frac{t_{i}^{\text {oce }}}{t} \cdot \frac{1}{\text { toce }_{i}} \sum_{t}\left(O_{i, t}\right) \quad \text { substitution by }(4.3) \\
& =\frac{1}{t} \cdot \sum_{t} \frac{S_{i, t}}{C_{i}}=\frac{1}{t} \cdot \frac{\sum_{t} S_{i, t}}{C_{i}} \\
& =\frac{\sum_{t} S_{i, t}}{t \cdot C_{i}} \text { or } \quad \frac{\sum_{t} S_{i, t}}{\sum_{t} C_{i}}
\end{aligned}
$$

and denotes the used fraction of the room's total seat hours.

In order to obtain total utilization it is necessary to combine that of all rooms. In the course of this it makes sense to weight the rooms, as their size (usually) differs. As such, the sum over all rooms' weighted utilization divided by the total weighting leads to

$$
U^{W}=\frac{\sum_{i} W_{i} U_{i}}{\sum_{i} W_{i}},
$$

with $W_{i}$ being the respective weighting for room $i . W_{i}=1 \forall i \in \mathcal{I}$ ( $\mathcal{I}$ being the set of all rooms) resembles the trivial unweighted case, but it comes natural to weight the rooms' utilizations according to room-capacity (i.e. $W_{i}=C_{i}$ ). Thus capacity-weighted (total) utilization is defined as

$$
\begin{aligned}
U^{C} & =\frac{\sum_{i} C_{i} U_{i}}{\sum_{i} C_{i}} \text { substitution by (4.6) } \\
& =\frac{\sum_{i} \varnothing_{i} \frac{\sum_{t} S_{i, t}}{t \cdot \not \varnothing_{i}}}{\sum_{i} C_{i}}=\frac{\sum_{i} \sum_{t} \frac{S_{i, t}}{t}}{\sum_{i} C_{i}} \quad t \text { being fixed } \\
& =\frac{\frac{1}{t} \cdot \sum_{i} \sum_{t} S_{i, t}}{\sum_{i} C_{i}}=\frac{\sum_{i} \sum_{t} S_{i, t}}{t \cdot \sum_{i} C_{i}} \quad \text { also written as } \\
& =\frac{\sum_{i} \sum_{t} S_{i, t}}{\sum_{i} \sum_{t} C_{i}}=\frac{B}{B_{M}} \text { by (4.1) and (4.2). }
\end{aligned}
$$

Subsequently capacity-weighted utilization is the ratio of seat-hours in the maximum number of seat-hours.

In practice generated schedules and lecture-to-room assignments are usually disturbed "when students arrive and populate the skeleton structure of the timetable", as McCollum (2007, p. 17) puts it. This subsequently causes conflicts and problems, which are usually solved "through negotiation and compromise" without the need for repeated generation of timetables.

Taking this into account, it is not (absolutely) necessary that an assignment of events to rooms is free of conflict. It can be sufficient to generate an assignment and produce a list of conflicting events which are then sorted out "manually" (i.e. negotiated). Thus the amount of conflicts within a solution of an event-room allocation problem poses an additional, practical measure for the goodness of it. Needless to say that conflict-free assignments are favored over such that inherit conflicts, but the possibility to also accept "imperfect" solutions and to compare (and rank) such solutions can be of great benefit. An example for this is when, due to constraints, no "perfect" solution may 
be obtained. Another one is that a solution with few conflicts may generate a "tighter" schedule than one without conflicts, in which case the remaining (spatial and temporal) areas can be used more versatile.

For this measurement $\mathcal{E}$ denotes the set of all events $e$ (within the system during the temporal interval of interest). Based on it are the definitions of the two subsets of satisfied and unsatisfied events

$$
\begin{aligned}
\mathcal{E}^{\mathrm{sf}} & =\{e \mid e \in \mathcal{E} \text { and } e \text { has an assigned room }\} \subseteq \mathcal{E} \\
\mathcal{E}^{\text {usf }} & =\{e \mid e \in \mathcal{E} \text { and } e \text { has no assigned room }\} \subseteq \mathcal{E}
\end{aligned}
$$

which are complements, i.e.

$$
\mathcal{E}=\mathcal{E}^{\mathrm{sf}} \cup \mathcal{E}^{\mathrm{usf}} .
$$

Accordingly the number of unsatisfied events is the cardinality $\left|\mathcal{E}^{\text {usf }}\right|$ and a solution's success rate

$$
R=1-\frac{\left|\mathcal{E}^{\mathrm{usf}}\right|}{|\mathcal{E}|}
$$

is then defined as the relative distance from a perfect solution (i.e. one where $\left.\mathcal{E}^{\text {usf }}=\emptyset\right)$.

The previously used terms space management and space planning denote the short-term allocation of available room (space management) and the strategical long-term coordination in order to meet demand (e.g. re-allocation of room for different tasks - space planning).

Room-time (RT) describes the option of using a room over a specified period of time (i.e. one or several time slices). For practical reasons RT is usually measured in units of room-hours (RH), which is one room worth of 60 time slices (of 1-minute length). RH are conceptually related to seat-hours (see (4.1) and (4.2)) and are used to articulate demand for room (with certain specifications). Formulated demand is referred to as requested room-hours ( $\mathrm{rRH}$ ). It is the task of space management to allocate room for $\mathrm{rRH}$ and (i.e. transfer the requests into booked room-hours (bRH)) and cope with emerging conflicts which arise if there are more requests than $\mathrm{RH}$ (with corresponding specification) available.

Room specifications include all "hard facts" describing a room. Most notable characteristics are capacity, type, infrastructure and location.

Lectures and courses are used synonymously. These terms suggest periodic repetition of dates - something not necessarily found with events in more general applications. Thus event is used to denote each individual date of reoccurring lectures as well as for singular dates. To hold an event adequate space is required, which means that (1) the event's requirements are (more than) met by the assigned room's specifications and that (2) sufficient RH are available.

Course credit (CC) is the weighting of the time requirements of an academic course as such it is used to compute the calculative course volume (CCV) which is the expected amount of RH needed - by multiplying all CCs by the number of dates, which is usually equal to the number of weeks $w$ per semester. For the MoreSpace it has been assumed that every courses is held once a week during the semester and thus $w=14$. The CCV makes it possible to compare articulated room demand to a reasonable estimate and thus identify existing discrepancies or anomalies. 
The calculated course volume is computed as

$$
C C V=\sum_{e} C C_{e} \cdot w
$$

where $w$ stands for the number of weeks in the semester (i.e. number of repetitions of the event - in the MoreSpace project $w=14$ ) and $C C_{e}$ for the course credit of $e$. The real-world counterpart to $\mathrm{CCV}$ is the total of requested room hours

$$
r R H=\sum_{e} \Delta_{e}(t)
$$

where $\Delta_{e}(t)$ is the number of time slices required for event $e$. In the ideal case the articulated demand should be equal to the calculated one, i.e. $C C V=r R H$. In practice there usually is a discrepancy. Its degree can serve as an indicator for potential anomalies.

Formally room-time is a set of pairs, defined as

$$
\mathcal{R}:=\mathcal{I} \times \mathcal{T}
$$

where $\mathcal{I}$ is the set of all rooms and $\mathcal{T}$ that of all time slices. As such, the total available room-hours $t R H=\frac{|\mathcal{R}|}{60}$ are obtained by standardizing (division by 60 ) the cardinality of $\mathcal{R}$.

The task of space management is, as mentioned before, to assign room to events and thus transform $\mathrm{rRH}$ into $\mathrm{bRH}$. The event-to-room assignment is thus a - neither injective nor surjective - function

$$
f: \mathcal{E} \rightarrow \mathcal{R}^{\Delta_{e}(t)}: e \mapsto\langle i, t\rangle^{\Delta_{e}(t)}
$$

mapping events $e$ from the set of all events $\mathcal{E}$ onto the set of room-time $\mathcal{R}$, where $\Delta_{e}(t)$ denotes the number of time slices required by $e$ and the projection is defined as

$$
\langle i, t\rangle=: i\left(e_{t}\right) .
$$

Since events are (defined as) single dates, they require one room per time slice. The case of one event being held parallel in several rooms is excluded by either interpreting that it is using a coupled room (i.e. two rooms that have been merged to form one room, which is the case if video-transmission is used) or by creation of a second event (e.g. parallel groups are referred to as independent events).

Most events will require more than one slice of room-time, for which two cases are to be distinguished. Either (1) it is possible that the tuple $\mathfrak{D}:=$ $\left(t_{j+1}, t_{j+2}, \ldots, t_{j+m}\right)$ of all time slices ${ }^{11}$ is being held in the same room

$$
i\left(e_{t_{k}}\right)=i\left(e_{t_{l}}\right) \quad \forall k, l \in\{j+1, \ldots, j+m\}
$$

or (2) that the room is changed once or several times (e.g. block courses with a different room before and after a break). For each change of room a new sub-tuple $\mathfrak{S}_{g} \subseteq \mathfrak{D}$ is defined, so that $\mathfrak{D}=\mathfrak{S}_{1} \oplus \mathfrak{S}_{2} \oplus \ldots \oplus \mathfrak{S}_{n}$ where

$$
\mathfrak{S}_{g}=\left(t_{j+x_{(g)}}, t_{j+x_{(g)}+1}, \ldots, t_{j+x_{(g)}+y_{(g)}}\right) \quad \forall g \in\{1, \ldots, n\}
$$

\footnotetext{
${ }^{11}$ It is obvious, that each event has its distinct tuple.
} 
and

$$
\begin{aligned}
x_{(1)} & =1, \\
y_{(n)} & =m, \\
1 & \leq x_{(g)} \leq m, \\
y_{(g)} & \leq m-x_{(g)} \text { and } \\
x_{(h)} & =x_{(g)}+y_{(g)}+1 \text { for } h=g+1,
\end{aligned}
$$

$\forall g, h \in\{1, \ldots, n\}$, such that

$$
i\left(e_{t_{k}}\right), i\left(e_{t_{l}}\right) \ldots\left\{\begin{array}{l}
i\left(e_{t_{k}}\right)=i\left(e_{t_{l}}\right) \quad \forall t_{k}, t_{l} \in \mathfrak{S}_{\mathfrak{g}} \\
i\left(e_{t_{k}}\right) \neq i\left(e_{t_{l}}\right) \quad t_{k} \in \mathfrak{S}_{\mathfrak{g}}, t_{l} \in \mathfrak{S}_{\mathfrak{h}}: g=h-1 .
\end{array}\right.
$$

Finally an event $e$ is formalized as $n$-tuple

$$
e=\left\langle C_{e}, \Delta_{e}(t), Y_{e}, i\left(e_{t}\right)^{\Delta_{e}(t)}, \ldots\right\rangle
$$

where $4 \leq n \in \mathbb{N}$. The reason for this is, that events can not only contain (the necessary) core information but also (an arbitrary amount of) additional information. As core information are regarded

- $C_{e} \ldots$ the minimum capacity necessary

- $\Delta_{e}(t) \ldots$ event-duration (i.e. necessary number of time slices)

- $Y_{e} \ldots$ required room type and

- $i\left(e_{t}\right) \ldots$ assigned room at time-slice $t$; a tuple of size $\Delta_{e}(t)$, where $i\left(e_{t}\right) \in$ $(I \cup\{\emptyset\})$,

while additional information can be such as type and name of event, preferences (e.g. for assigned room), curricula to which the event belongs, group information, organizing unit, etc.

\section{Questions the Model Should Answer}

As laid out in chapter 3, for the outcome of a simulation-project to be beneficial it is essential that its objectives are worked out and specified exactly. In the case of the MoreSpace project at TU Vienna, three basic application areas have been identified (see page 104). For each of these areas the following lists of questions have been elaborated which are to be answered by the model and thus guided model implementation.

1. For testing of room blocking scenarios (due to reconstruction in the course of Univercity2015) the questions were:

- Is the chosen blocking scenario (along with current space management) compatible with teaching activities?

- Can all events be carried out (i.e. is it possible to assign a room to every event)?

- Are the rooms assigned of required size?

- Are rooms assigned of required room type? 
- Are sufficient space capacities available?

Answering this question requires specification of a threshold level by the respective decision makers.

- In the case that a scenario is not acceptable, following results shall assist finding a better solution:

- How many events could not be assigned?

- Which event types do experience shortages (lectures, tutorials, laboratory tutorials, etc.)?

- At which times do bottlenecks occur?

- Which room sizes/-categories are struck by bottlenecks?

2. For generation of allocation proposals (i.e. proposals for event-to-room allocation that are then to be approved -or not- by responsible decision makers) for events of mandatory courses (for semesters 1-4 and 1-6 respectively), these were:

- Is it possible to assign adequate rooms to all events of mandatory courses, or do events exist which cannot be satisfied (unsatisfied events)?

And, if unsatisfied requests exist:

- How many requests could not be satisfied?

- List of unsatisfied requests ("conflict list")

- Are bookings within dean's specifications (e.g. room utilization within limits)?

And, if not: Which temporal intervals are the bottlenecks that cause utilization to be below limits/specifications?

3. For retrospective analysis of the system (i.e. simulation using historical data) and testing of varying space management strategies:

- Which space management strategy (e.g. order and priorities of eventto-room assignment) causes how many unsatisfied requests?

Priority can be given to ...

- large events (i.e. many students) - from large to small

- events of block courses

- events for which a room with (perfect) size is available (i.e. attending students $=$ capacity)

- single events

- What effect do fixed rooms (for mandatory lectures) have upon ...

- occupancy of rooms

- frequency usage of rooms

- utilization of rooms

- number of unsatisfied requests

- Which effects does the change of rooms (splitting/merging) over time have?

- Assessment

- number of unsatisfied requests 


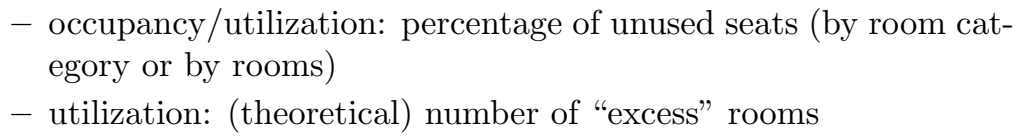

\subsubsection{System Analysis of TU Vienna}

System analysis is a process that focuses on three different - to some extent interweaved - layers of the system (psycho-social, organizational and technological layer; see section 3). Here the tools previously presented will be used to (a) analyze the stakeholders and extract the key-stakeholders for the MoreSpace project (attributed primarily to the psycho-social layer, but also to the organizational one). Based on the identified key-stakeholders the (b) processes of and for the room-booking-process will be investigated for the three application areas previously defined (attributed to the organizational layer). Finally (c) the assessment of necessary data (source/location, quality, etc.) will be carried out (primarily attributed to the technological, although with aspects of the organizational layer).

\section{Stakeholder Analysis (SHA)}

According to the process for stakeholder analysis (laid out in section 3.1.2), definition of the projects goals forms its basis - which is completed with the above specification (see section 4.1.1). It is followed by an extensive search for all stakeholders involved - be it apparent or not. For the MoreSpace project, taking place at a public university, aiming at the use of buildings owned by a federal company while still under (indirect) governmental control, the number of stakeholder-groups is, as expectable, high.

These stakeholders (and groups of stakeholders, respectively) are then analyzed with respect to the relevant criteria. As proposed, interest and power, with respect to the project and its goals, are used to classify the identified stakeholders. As the project team was composed from within the system itself, it did rely on its own, tacit knowledge for the classification. Needless to say, that this saved a vast amount of time that otherwise would have had to be spent on research and collection of respective information.

The following list briefly characterizes the identified stakeholders according to their (official/formal and personal) goals, their requirements, chances, risks and the assessed power and willingness for cooperation regarding the project. The latter are rated from 1 to 10 with 10 being the highest (power and interest for cooperation). Their order is arbitrary, with the exemption of the last four, which are external stakeholders (i.e. not from inside TU Vienna).

1. Rectorate

- Goals: create a good image and branding; increase in quality; fulfilling economic needs

- Requirements: fulfillment of quality measures; protection of places for students

- Chances: efficient assignment of resources; enable teaching activities with available resources

- Risks: lack of vision and motivation 
- Power: 10

- Interest: 10

2. Department for building, construction and technology (GuT)

- Goals: efficient handling of available resources

- Requirements: improvement of utilization and thus finances

- Chances: efficient utilization

- Risks: high costs through inefficient utilization

- Power: 6

- Interest: 8

3. Room-allocation group

A working group at TU Vienna concerned with lecture-to-room assignment. The group is an informal body that consists of staff from the "Department for Studies and Examinations" and the faculties' deans. It tries to coordinate inter-faculty assignments and solve eventual conflicts.

- Goals: Coordinate and satisfy room requests

- Requirements: enable teaching activities

- Chances: reduction of strain; increase of efficiency

- Risks: coordinate and consider many (and conflicting) interests

- Power: 8

- Interest: 6

4. Lecturers

- Goals: career; publications; obtain acceptance; social contribution

- Requirements: good surrounding conditions for research and teaching

- Chances: efficient room allocation according to needs

- Risks: lack of cooperation; cumbersome administrative machinery; friction due to undefined competences

- Power: 8

- Interest: 8

5. Students

- Goals: qualitative education

- Requirements: sufficient room for qualitative teaching

- Chances: receive teaching in adequate room and infrastructure

- Risks: loosing autonomously administered spaces

- Power: 4

- Interest: 4

6. MoreSpace project-team 
- Goals: implement and deploy MoreSpace at TU Vienna; acquire financing for employees; generate publications

- Requirements: scientific input; cooperative stakeholders

- Chances: exploitation rights; publications

- Risks: exploitation rights; publications; functionality

- Power: 10

- Interest: 10

7. Zentrum für Innovation und Technologie (ZIT)

A funding authority (for innovation and technology) of the city of Vienna, which funded the MoreSpace project.

- Goals: support research and development in Vienna; strengthen Vienna as an innovative business location

- Requirements: financial support of research topics; generation of technological advances

- Chances: improvement of Viennese economy; bring innovations onto the market; generate synergy effects (i.e. reduce government spending on room through subsidies for research)

- Risks: bad investments; failed projects

- Power ${ }^{12}: 10 ; 3$

- Interest: 10

8. Federal Department for Science and Research

- Goals: create and provide framework for research, science and teaching

- Requirements: decision- and policy-making for education and research including administration of financial resources

- Chances: save money for building-related costs; improve quality of service; obtain acceptance

- Risks: political interference

- Power: 5

- Interest: 3

9. Municipality and urban administration

- Goals: enforce compliance with miscellaneous provisions; keep universities in Vienna; generate positive effects from universities for the city

- Requirements: unclear

- Chances: bonding to location; synergy effects for municipality (e.g. putting results into use for city-owned/-administered buildings)

\footnotetext{
${ }^{12}$ The ZIT acted as funding authority and thus its power is assessed into "before" and "after" funding was granted. In the former case, with funding being a neck-breaker, the ZIT's power was 10. After funding was granted power decreased as the agency is not directly involved in the project and "only" interested in a successful outcome.
} 
- Risks: resettlement; political interference;

- Power: 3

- Interest: 2

10. Bundesimmobiliengesellschaft (BIG)

A state owned real estate company providing the buildings used by TU Vienna. See section 1.2.4 for the BIG's conflict of interests.

- Goals: high revenue

- Requirements: optimization of costs and utilization

- Chances: high utilization of federal buildings

- Risks: bad investment, loss in value

- Power: 2

- Interest: 3

This classification, especially for stakeholder groups (i.e. rectorate, lecturers, students) is of course a general one. The attitude towards the project within such a group can vary drastically, depending on personal preferences and experiences. A very good example for this is the "room allocation group". It is extremely heterogeneous, as it consists of the deans of all faculties, which all have a different background as well as differing faculty-specific problems. For example does the faculty of (technical) chemistry only have very small student numbers but very specific room demands (i.e. specialized, expensively equipped laboratories) while the faculty of architecture has huge numbers of students and thus is in need of large auditoria and student-workplaces (where those can draw and construct models). In addition the coordinators of the whole group are staff from the "Department for Studies and Examinations", which is a part of the rectorate.

Taking the power- and interest-data from the above assessment, one can produce a scatter plot of the stakeholders (see Fig. 4.1). In this plot the stakeholders in the top right corner are those who are most critical for the project.

As visible, five players are within the most important (high power, high interest) category: the rectorate, the GuT, the room-allocation group, the lecturers and finally the MoreSpace-team itself. These are the system's key-stakeholders. The remaining stakeholders are of lower interest and power (with one exception being the ZIT). Nevertheless, they must not be ignored, but it is sufficient, that they are monitored and taken into consideration to prevent damage.

The ZIT is, as mentioned, an exception. This player's case is special, as its role is changing at the point where it grants funding for the project. At this point in time the power to influence the project reduces drastically. The reason for this is that at that point the funding contract is being signed and thus surprises and changes in attitude are not possible any more - except if the project would get off track and funding would have to be reimbursed. Although in this case the stakeholder would again increase in power and thus shift from the "keep informed" into the "manage closely" category.

\section{Definition of Business Processes}

Analysis of the room assignment process proved to be more complicated than the SHA. The reason for this is, that there was no formal process(es) for room 


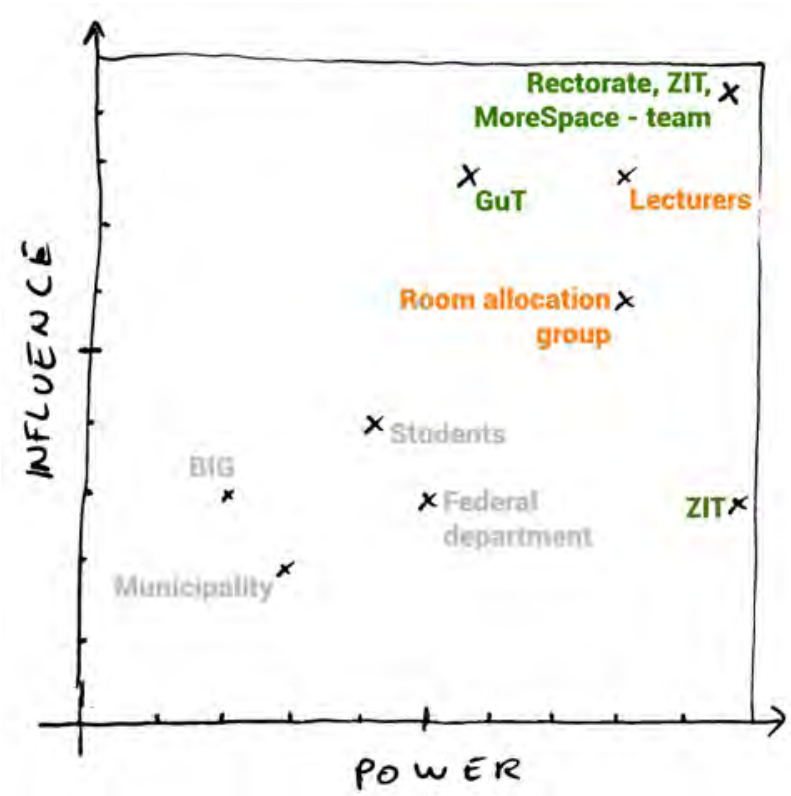

Figure 4.1: Analysis and classification of stakeholders encountered within the MoreSpace project (green - positive; orange - sceptic; gray - neutral).

allocation at TU Vienna. The processes, if formalized at all, were connected to (groups of) rooms, which again were assigned to organizational units. Thus the organizational units were in charge of room allocation for "their" lecture rooms ${ }^{13}$. Subsequently availability of these rooms for outside-events (i.e. events organized by other organizational units than the room's "owner") was (very) limited. As an example: it was only possible to book a room on short call, but one would have to do this via the "owner's" secretariat, which might or might not be manned at the time necessary. And only a few, usually large or representative, rooms were actually "centrally" managed (i.e. by the "Department for Studies and Examinations" which is a sub-division of the rectorate).

Since formalized business processes are necessary in order to deploy the MoreSpace-tool and embed it into the booking system, it was necessary to define them for the TU Vienna. With the tool aiming at three different scenarios (see section 4.1.1) three different processed were required, taking into account the respective scenarios' peculiarities. Thus three business process models (BPM) were developed within the MoreSpace project and presented as proposals for reorganization of the (non-) existent processes.

For all three scenarios the involved actors are the same, although their role varies greatly. The previously identified key-stakeholders were used to counter check the players identified for the business processes, and they are more or less identical - although with two differences. Firstly, the rectorate and the room-allocation group are substituted by general "decision makers", which even might be represented by deans. Besides this, the GuT is entitled to specify thresholds and control the space management strategies. The reason for this

\footnotetext{
${ }^{13}$ In the following quotation marks are used for possessive terms as to indicate that the room is not really owned by an organizational unit.
} 
is the assumption that the GuT, as a sub-division of the rectorate, enforces the rectorate's policies. With this interpretation it can be avoided to add a $6^{\text {th }}$ player to the BPM, which only serves as a policy maker. The second change from the key-stakeholders is the introduction of a "virtual player": the underlying IT-system.

At TU Vienna, as a result of historical development, various isolated ITsystems existed which have been integrated step-by-step into a holistic system called "TISS" (which stands for "TU Wien Informations-Systeme \& Services" which translates into English as "Information Systems and Services of the Vienna University of Technology"). This system is administered by the ZID (not to be confused with the ZIT!), the universities IT service provider. It is intended to include all electronic services which are offered by the university, ranging from student self services (e.g. printing reports and certificates, registering for lectures) via e-learning courses, project management up to the possibility for room reservation. This system is deemed as the "information backbone" for the MoreSpace project, serving as central data-base (storing room- and eventinformation) as well as the user-interface for lecturers, decision makers and students.

The BPM for scenario (1) - testing of room blocking for reconstruction (or alternatively testing of system limits or new curricula) - is depicted in Fig. 4.2. As visible, the most active stakeholder in this scenario is the GuT, which initiates, terminates and controls the whole process ${ }^{14}$. The GuT defines (reasonable) blocking scenarios and schedules, reference values and then orders simulation (and and post-processing) of the assigned tasks. If the results comply with the reference values the scenarios are accepted and reconstruction scheduled accordingly. Subsequently the information regarding room blocking is saved within the TISS and according notice passed to the decision makers and lecturers. These again have the possibility to issue feedback, which is collected and passed on in a condensed form by the decision makers (i.e. they act as facilitators).

In case that the results are not satisfactory, it is necessary for the GuT to either modify the settings (e.g. order of blockings) or the parameters (e.g. reduction of thresholds and thus tolerating more conflicts). Then the simulationevaluation process is entered again with this new input.

As could be expected, the business process for scenario (2) - real-time space management - it the most complex one. In this process (depicted in Fig. 4.3) the GuT acts more or less as walk-on, as it only supplies information regarding rooms. This scenario also intends the use of "external" simulation parameters (i.e. booking strategies to be applied) which are indicated by the external arrow (entering at the upper right corner). The idea behind this is, that the simulation parameters and booking strategies are evaluated using scenario (3) and the most appropriate ones chosen for the simulation of this scenario by the decision makers.

Scenario (2) is kicked off by lecturers offering their lectures, which is equivalent to creation of these courses (consisting of one or several events) within the TISS. As explained above, the TISS serves as user-interface (i.e. the lecturers enter all relevant information via web-GUI's) and information backbone (in this case all course- and event-information is stored in it). The next step within the

\footnotetext{
${ }^{14}$ This might change if the purpose would be to test the system's limits or new curricula. In these cases the decision makers (responsible for teaching activities) would take the position of the GuT.
} 


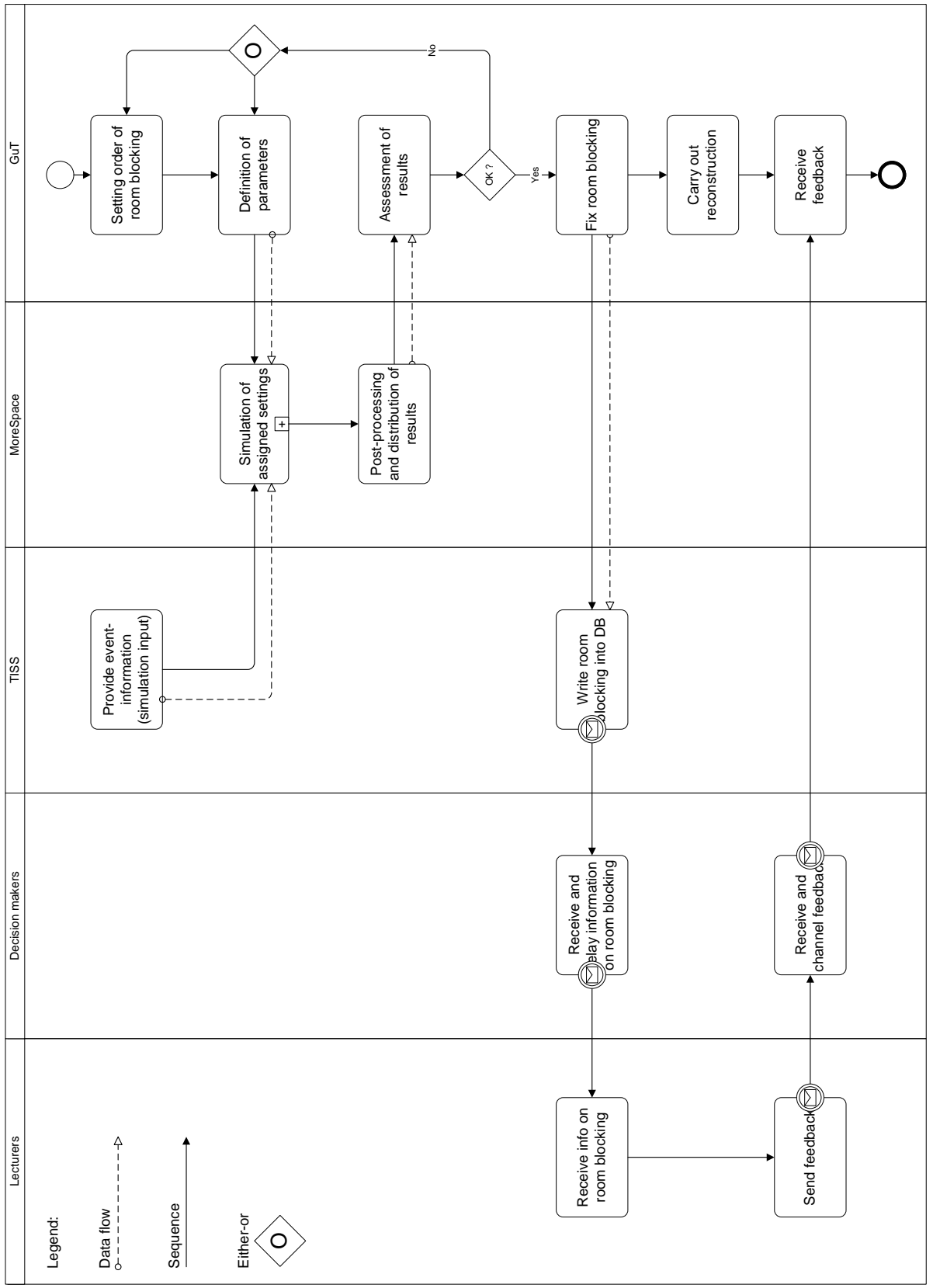

Figure 4.2: Business process model of scenario (1): testing of room blocking strategies (notation: modified BPMN as described in sec. 3.1.1).

$\mathrm{BPM}$ is the decision makers' check of the course-information and its subsequent release or release-denial, depending on the quality and eventual conflicts. If conflicts exist, the decision makers are intended to resolve them on their own and thus modify course information within TISS accordingly. The lecturers offering affected courses are then notified and have the chance to either accept or change 


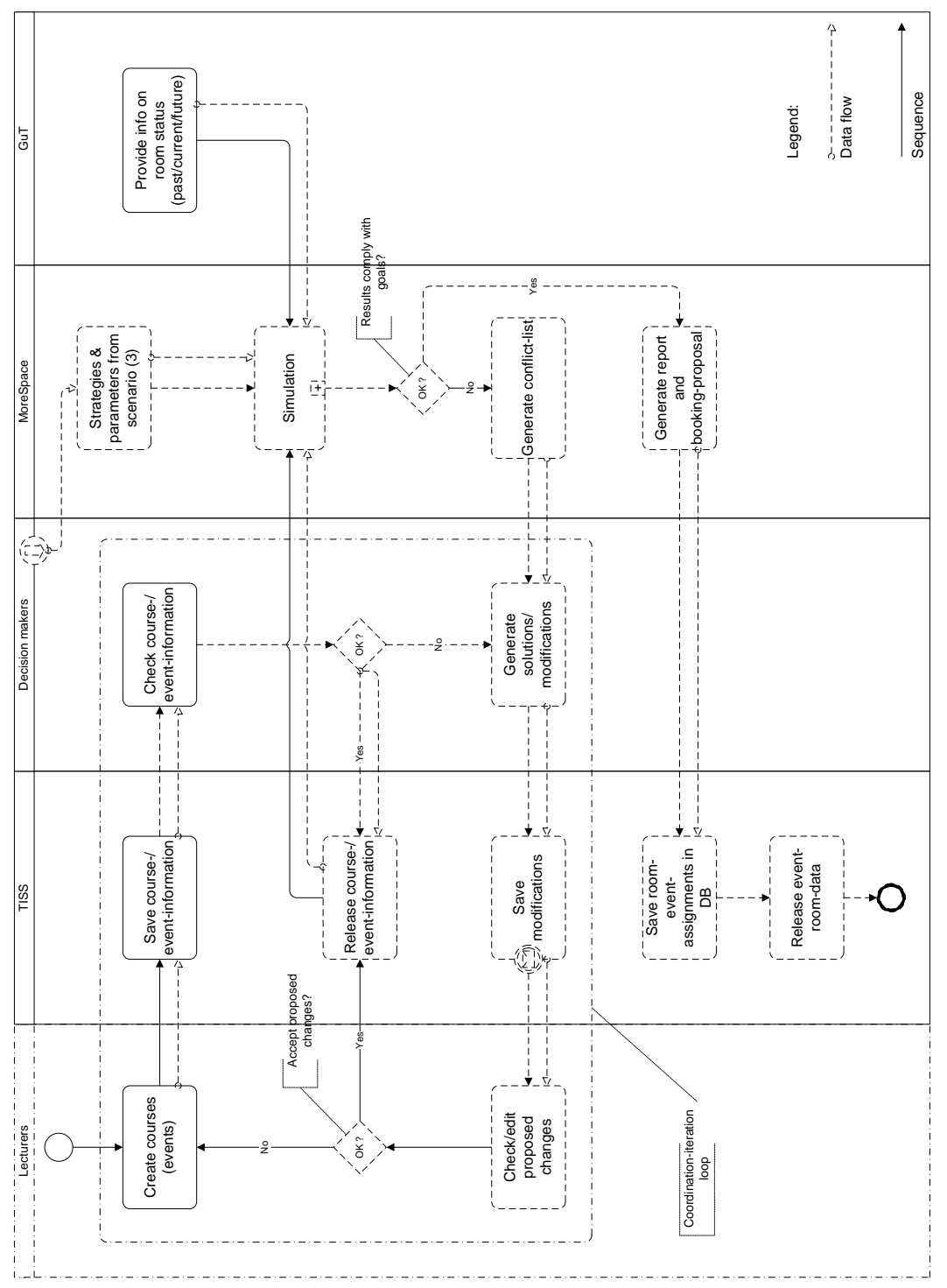

Figure 4.3: Business process model of scenario (2): real-time space management, i.e. assignment of events to rooms (notation: modified BPMN as described in sec. 3.1.1).

the decision makers' "proposal". This procedure is repeated until finally green light is given by the decision makers to proceed.

With the data within the TISS and with (room-related) input from the GuT and specifications regarding space management strategies, simulation of the room-allocation process is carried out. If the generated event-to-room as- 
signment complies with the set targets, this assignment is passed on and saved within TISS, from where it is accessible to the broad audience. If on the other hand the proposed assignment does not fulfill the objectives a conflict list is generated and handed over to the decision makers in order to sort these out. With this the initial loop of conflict-management is entered, and the same procedures apply again until successful generation of an assignment list.

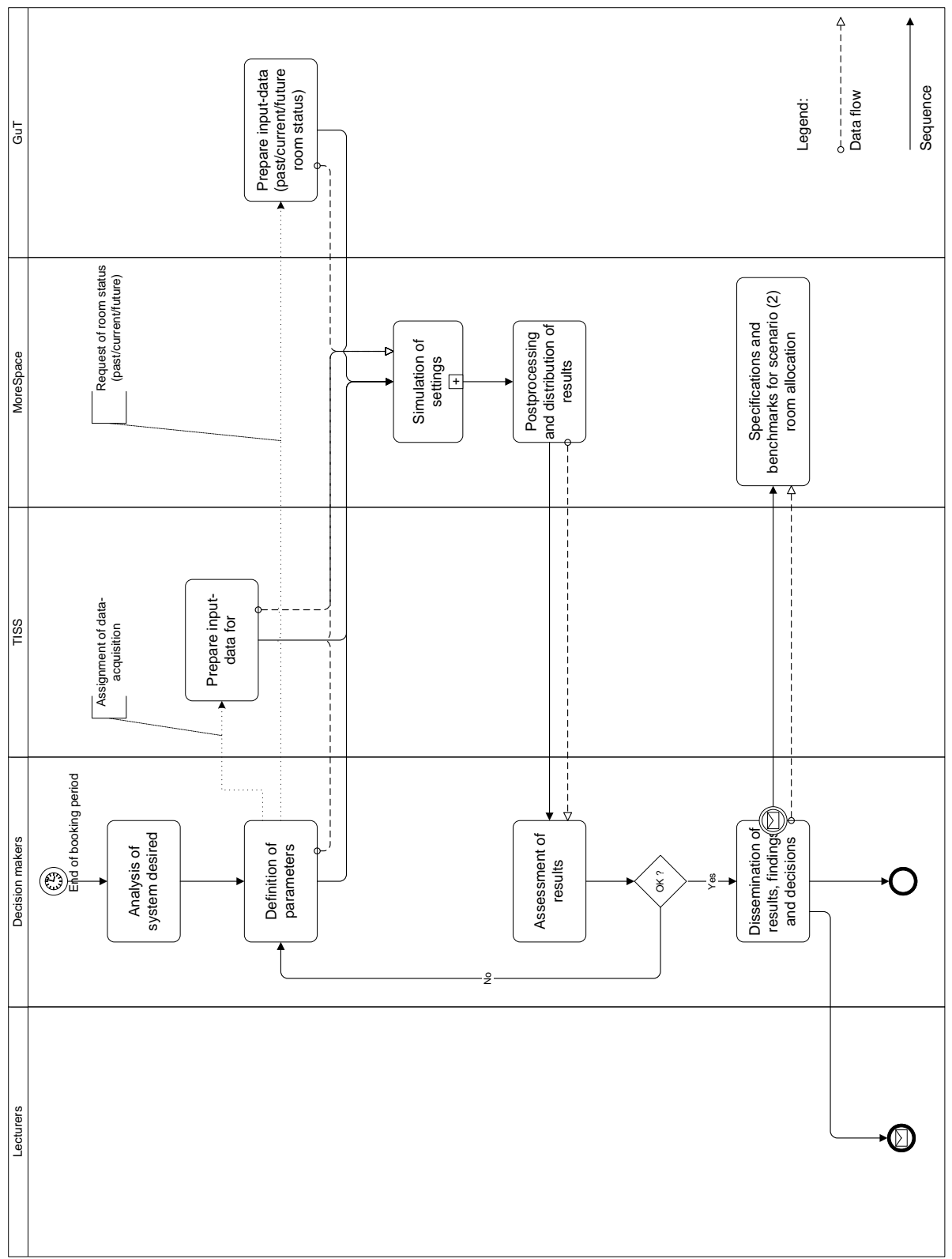

Figure 4.4: Business process model of scenario (3): retrospective analysis and testing of space management strategies (notation: modified BPMN as described in sec. 3.1.1).

The final scenario (3) - retrospective analysis and testing of space manage- 
ment strategies - can, as pointed out above, be used in order to identify suitable space management strategies which are then applied to scenario (2). The third scenario (depicted in Fig. 4.3) is initiated by the decision makers who are interested in an analysis of the system. They initiate scenario (3) after the end of a booking period - in most cases the end of a semester - and specify the aspects that are to be analyzed and the parameters to be used for simulation. These specifications are passed on to the simulation and the data necessary is made available for the simulation by the TISS and GuT. Depending on the simulation goals data can be either from the past, current, predictions for the future or a mixture of those.

Subsequently MoreSpace carries out simulation of the specified settings and post-processing of the results, according to requirements of the decision makers. These are passed on to the decision makers and evaluated by those. If the outcome is unsatisfactory, then it is up to the decision makers to define additional variations and "send" them to simulation - thus the procedure described above is repeated. If, on the other hand, the outcome is satisfying, then the decision makers can generate policies for scenario (2) based upon them. In addition the lecturers are informed about the outcome, new policies and implications.

\section{ERD - technical layer \& organizational}

From the BPM it is visible, that the MoreSpace tool is fed with data by the TISS, the GuT and parameters coming from decision makers. The structure of the required input data is the same, regardless of the intended scenario for which it is to be used - only the content and the aspects are changing.

In order to identify the data that has to be provided by these systems/ stakeholders, an entity relationship diagram (ERD) is set up for the model. Its development was an evolutionary process within the project. It set out with the identified entities necessary for the model. In its basic versions these are students, rooms and courses. Although, at a closer look, the latter ones split up into two more parts: groups and events of the courses, with each of these five entities having its own set of attributes.

As shown in Fig. 4.5, the relationship between courses and its groups is a $1-(1, n)$ relationship, meaning that each course can have one or several groups (e.g. study groups). Each of which itself has a $1-(1, n)$ relationship with events. Thus each of the courses' groups can have one or more associated events. Naturally each event requires a space in which it is held. The $1-(1, n)$ relationship between events and rooms allows it to accommodate large events in two rooms, which are then connected via video-conferencing.

Originally the student was assumed to be connected to the entity course (via $1-(1, n)$ relation). As such, every student can subscribe to multiple courses. But this interpretation is shifting depending on the organizational point of view. It is also possible to argue that the students are not in a relationship with the courses but directly with one of its groups. This reflects the shift from a holistic view to the practical perspective of lecturers that actually organize the courses and their groups, while holistic decision makers are only interested in balanced (and reasonable) ratios of students for each of the courses' groups.

From a room-centered perspective (as it is assumed during simulation) a third interpretation is possible: students are connected directly to events. With this it is possible to reflect students dropping out of courses or missing events 


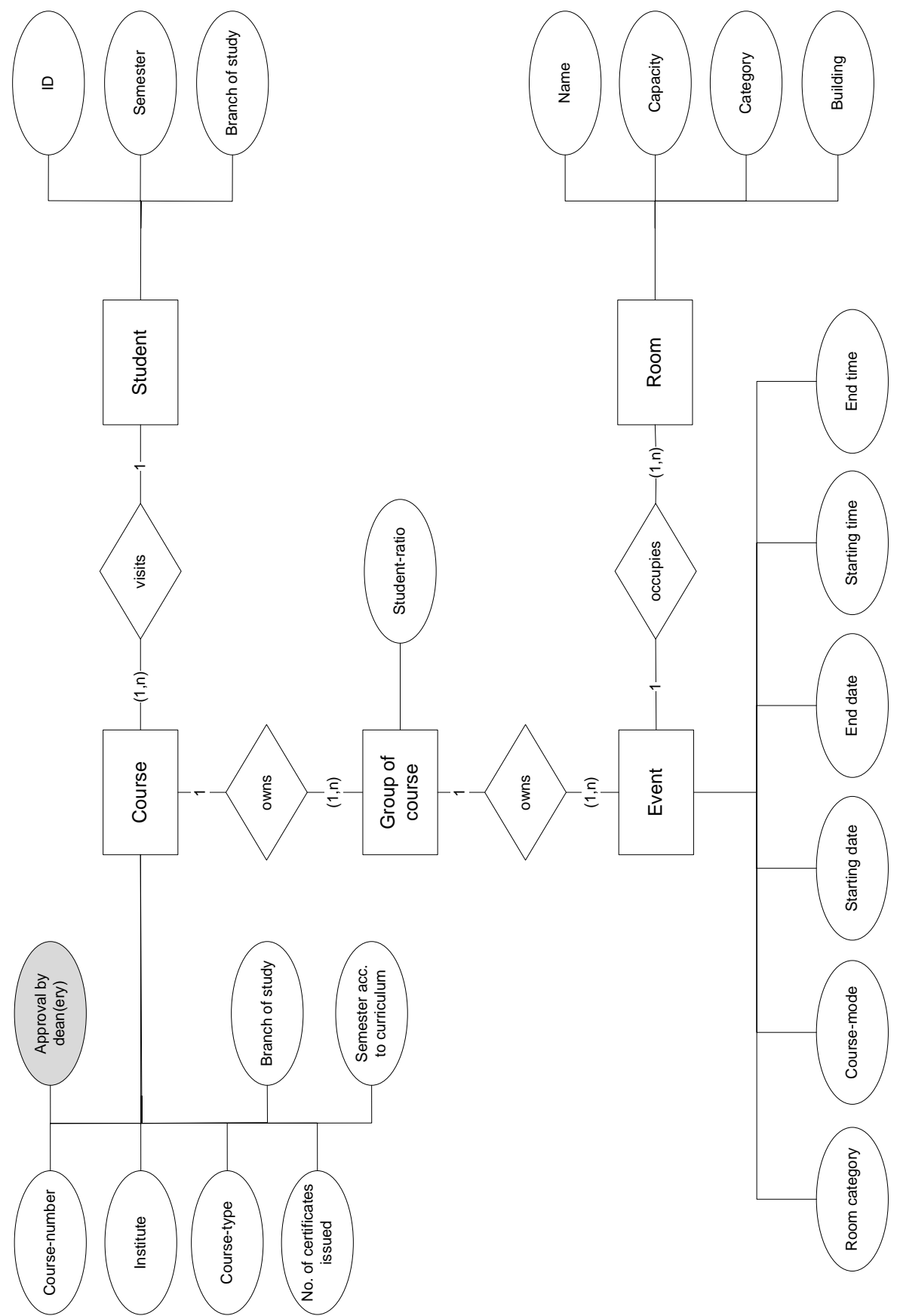

Figure 4.5: Entity relationship model with three main (students, rooms and courses) and two sub-entities (groups and events of courses).

due to various reasons (e.g. too long travel-times between lectures or temporally conflicting schedules). Nevertheless this perspective is of no or little relevance to the lecturer. The reason for this is that (within the Austrian university system) 
students enrolled in courses with compulsory attendance receive a negative grading if dropping out. Thus they are still "in the course" from a lecturers point of view, even if they don't attend any longer. For lectures and courses without compulsory attendance grading is given only after students take the respective exam. Subsequently it is irrelevant whether they attend the course or not and their "enrolling" for the course is only of informative nature.

In theory even a fourth interpretation, where students are connected to rooms, is possible. This would allow students to visit rooms at any given time without any connection to the events taking place in the rooms at these points in time. Subsequently it would be possible that such students block seats that are needed by students associated with the events. Since this situation is not a realistic one (students usually leave rooms when a course is being held that they are not associated with), it is not pursued further.

As noted above, the ERD evolved over time. It started out with the one in Fig. 4.5 which represents the holistic perspective of decision makers, i.e. with the entity student being connected to the entity course (in an $1-(1, n)$ relation). But over time, and especially for the implementation of the model, a more detailed ERD that reflects the system's behavior during simulation was required. The final ERD which fulfills these requirements is depicted in Figs. 4.6 and 4.7.

In the ERD one notices several major changes. Firstly the above entities are partially renamed or omitted: students are now persons, rooms became spaces, courses and groups are omitted and completely substituted by events. Secondly a lot of additional entities are introduced, such as event_has_person, which serves as a connecting entity between persons and events, booking which links events to spaces, category which determines the necessary room category of events and thus links those with spaces, organisation which on one the hand organizes events and on the other hand "owns" spaces. Spaces are connected to the entities building and, in a self-referencing way, to traveltime - an entity that specifies the time it takes to travel between two spaces (i.e. lecture rooms). The entities result with connection to (event, space,) usage and experiment which itself is connected to scenario and parameter, finally constitute the output of the model.

When comparing this ERD-version to the first one, it becomes evident, that several of the newly added entities are attributes of the early entities (e.g. course's attribute institute became entity organisation - and is now also connected to space; room's attribute category became an entity, etc.). Of course the perspective has also changed: from decision makers' holistic point of view to the data-base driven one, necessary for model-internal organization of entities. Both of which are helpful for analysis of the system and subsequently deliver valuable insights for deployment of the tool.

\subsubsection{Problems Encountered}

In the course of the previously described analysis several problems were encountered (e.g. skepticism of staff, incomplete data, problem to change processes, etc.). These are described in the following and will be used subsequently to assess the system's compatibility with the project-requirements. 


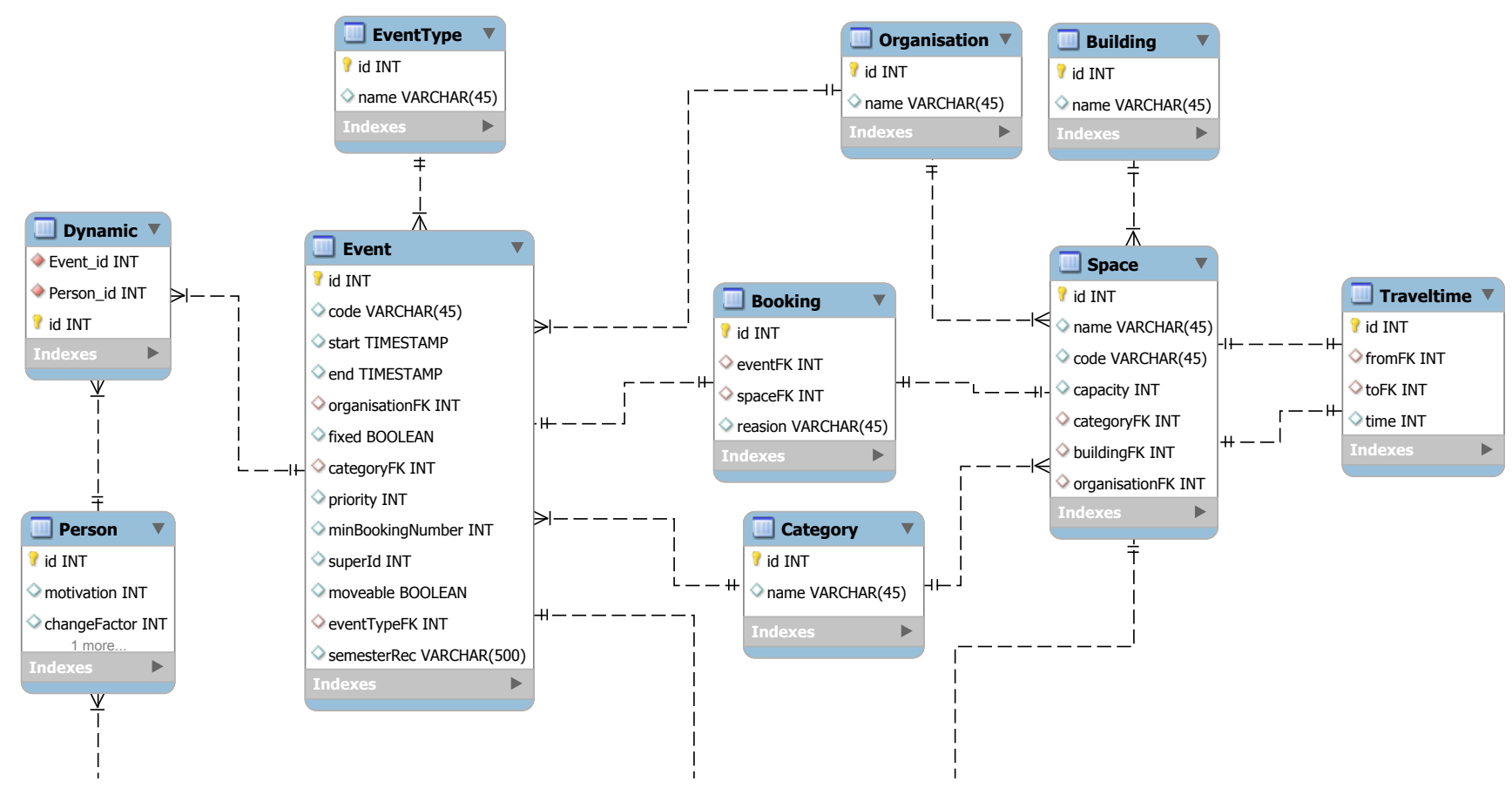

Figure 4.6: Entity relationship model of the data-base structure implemented within the simulation model (part 1 - input \& model-internal database).

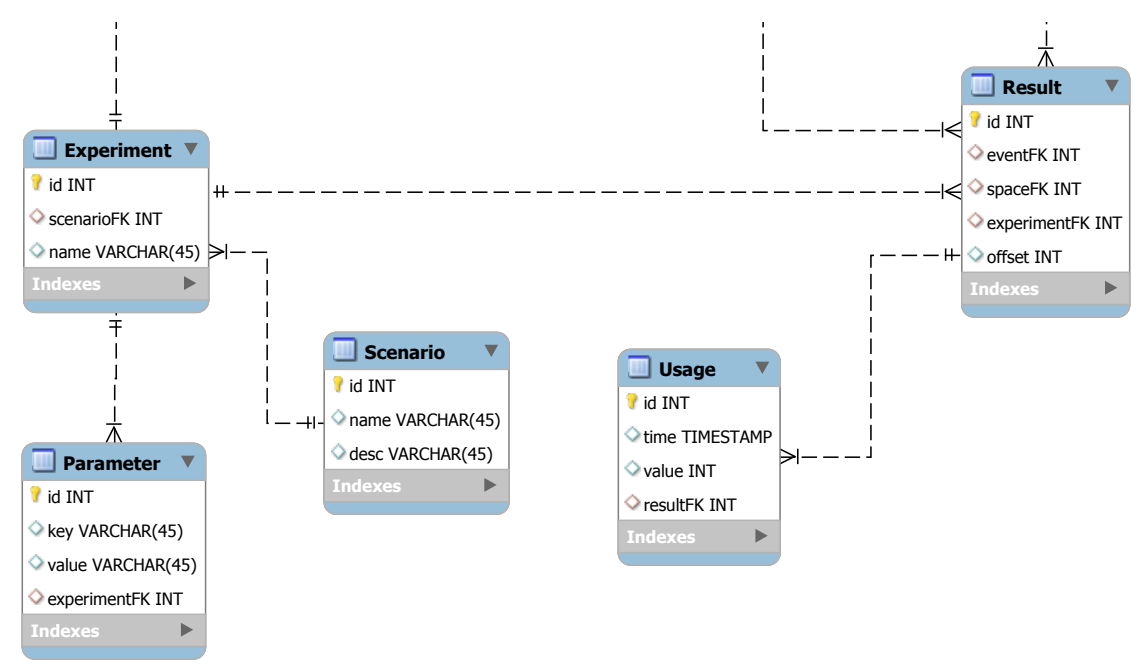

Figure 4.7: Entity relationship model of the data-base structure implemented within the simulation model (part 2 - model output).

\section{Data Assessment}

The data required for model-deployment is specified by the ERDs — from a general perspective (see Fig. 4.5) as well as from the model-internal database 
(Figs. 4.6 and 4.7). Thus it is obvious that data is needed to model the students (with their behavior), the rooms and the events. At project start the TISS was still under construction and only its predecessor (TUWIS++) working. The TUWIS ++ (and complementary systems) have been in service for (partially) up to 40 years. Correspondingly the code was a wild agglomeration and subsequently the functionality and use as an efficient database not up to date. Step-by-step roll-out of the TISS, which bundled, replaced and enhanced the features of the previously existing systems, started in 2008 but it was not before fall 2010 that handling of events was also integrated. As a result provision of event-data was anything but straight-forward at the MoreSpace project's initial stage. Over the course of the project this situation changed (with roll-out of TISS) but it still remained unsatisfactory.

To obtain all course information necessary, the ZID had to extract three different data-sets from their systems, which were transmitted as csv- (or more accurately tsv-) files by mail. Data was obtained for two semesters: winter semester of 2007 and summer semester of 2008, which were submitted as one batch (i.e. the three data-sets contained information of both semesters). Such data-sets were received three times, with the first one containing all courses associated to the faculties for chemistry and mechanical and industrial engineering, the second contained those of architecture and of civil engineering and the final set contained the data of the four remaining faculties (mathematics and geoinformation, informatics, physics, electrical engineering and information technology).

Of the three data-sets the first contained the dates/periods of the courses, which were specified as intervals with date-type of the course (single event, repeating, blocked, etc.), starting date, ending date and starting and ending time, accompanied by the course number and the semester of the course. The second file contained, besides course number and the semester in which it was held, information for which programs of study the courses are intended (index numbers) and the nature of the course (mandatory, optional or elective course). Within the second and third batch (i.e. for all faculties except chemistry and mechanical and industrial engineering) it also included the number of the course's offering institute. The third file contained information on student-numbers, albeit only as number of examinees and number of exams given (which sometimes diverge slightly), in addition it also had the type of the course (lecture, seminar, excursion, laboratory tutorial, etc.) and again course number and semester.

In order to make sense of the data it was necessary to concatenate the three data-sets. It can be assumed that some course-information is not changing over time. This information includes type of the event (lecture, seminar, ...), intended branch of study, course-offering institute and the like. Other information such as dates, times and student numbers (examinee numbers, to be precise) are subject to change. Subsequently concatenation of course-information depends on the intended use. In order to obtain a first overview, based only on the not-changing information, one can use information from both semesters. If the goal is to extract the student behavior and student input-parameters for the simulation, one has to differentiate between winter and summer semester.

First analysis of data was undertaken by focusing on not-changing information. Thus the only aspect was to identify courses (regardless of semester) with incomplete information (although including temporally dependent data entries). This assessment (see Fig. 4.8) made it obvious that the quality was anywhere 


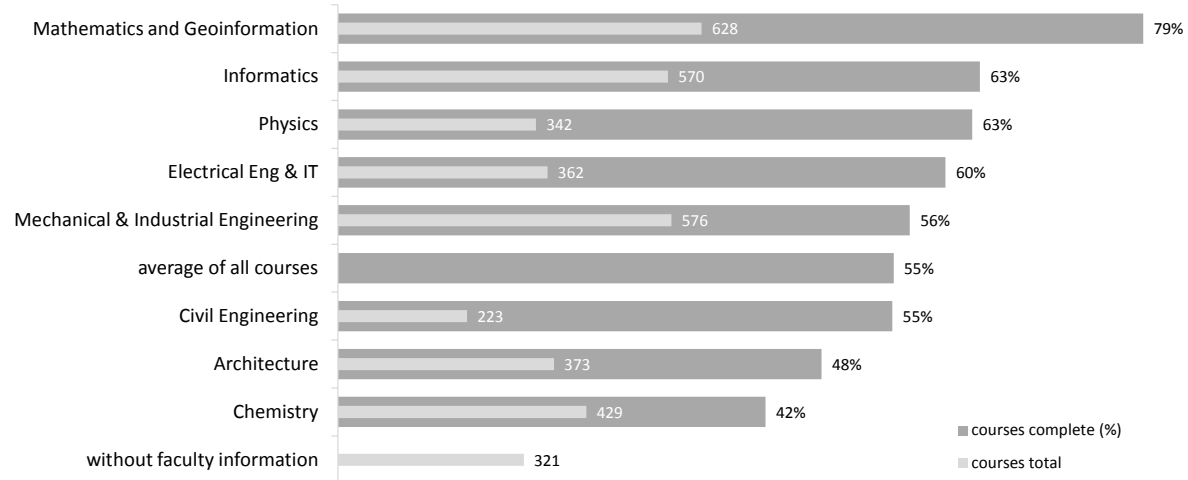

Figure 4.8: First quality assessment of course information, sorted by faculty (thick dark bars: ratio of courses with complete information; thin light bars: number of courses assigned to faculty).

near acceptable, although the quality varied greatly depending on faculty. For two faculties the initial data received was incomplete for more than $50 \%$ of their courses and seven out of eight faculties had incomplete information for more than $1 / 3$ of their courses. Even the faculty with the best data available had more than $20 \%$ incomplete courses. Over all courses offered, including those that could not be assigned to a faculty the average "completeness ratio" was a mere $55 \%$ — slightly more than half.

As there was no information about the "offering institute" for data from the faculties of chemistry and mechanical and industrial engineering, for these an assignment was made, based on course numbers ${ }^{15}$. It has to be noted, that it has been accepted, that this assignment is not completely correct. Nevertheless, the "courses without faculty" (lowest bar in chart) are only from the remaining six faculties. These courses either had no "organizing institute" entry or a number which could not be associated to any faculty.

In order to improve the quality a manual two-step repair-strategy was pursued. The first step was to look up each incomplete course in the TUWIS++ (via its web-interface) and - if available - obtain the information from there. It is needless to say that this tedious process of looking up, comparing and transcribing large quantities of numbers is an error prone one. In the second step the incomplete courses were handed on to the faculties' deans for analysis and improvement. In this context it is worth noting that (many of) the deans were very cooperative and interested in this process. The reason for this was according to their own account - that they also had only limited information and thus a great desire for an improved insight - and overview.

Nevertheless more or less problems remained, depending on the faculty. For some faculties this procedure was able to push the ratio of incomplete course information from an initial 50\% down to $32 \%$ (combined data-set for the faculties of mechanical and industrial engineering and chemistry). For others it was only of little success, as for example for the faculty of architecture, where

\footnotetext{
${ }^{15}$ As a rule of thumb the first three digits of the 6-digit course number denote the organizing faculties.
} 
incompleteness could be reduced only from $48 \%$ to $45 \%$ (none of which by help of the deanery). An interesting effect of the interviews with the deans was the identification of faculty-typical reasons for incomplete data. E.g. for chemistry the use of laboratories: since laboratories are extremely limited (by number as well as by type of use determined by their configuration) their use is very wellcoordinated among the involved institutes - often bypassing the TUWIS++ database. Analog "standard-problems" were found for almost all faculties.

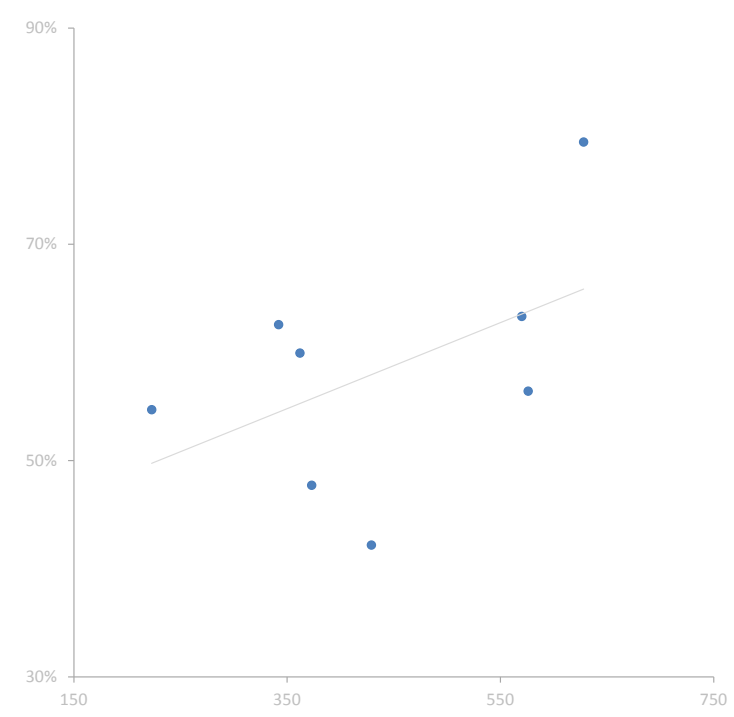

Figure 4.9: Number of courses offered (x-axis) vs. ratio of complete courses (y-axis) for faculties with linear regression ${ }^{16}$ (grey line).

Taking a look at the correlation between a faculty's total number of courses offered and its ratio of complete courses, Fig. 4.8 seems to exhibit a linear relationship. In Fig. 4.9 the numbers of each faculty's unique and complete courses are plotted against another. Arguably the sample of only eight faculties is not a highly significant one, nevertheless the linear regression (see line drawn in chart) supports the assumption that larger faculties (i.e. such offering a higher number of courses) tend to be better organized ${ }^{17}$.

As a consequence of the (poor) data quality, the project-team came up with a list of proposals for improvement of the graphical user interface (GUI) for creation of courses which would guide and require the user to enter more and complete data. Unfortunately these proposals were not implemented.

While course information was supplied by the ZID, the GuT was responsible for providing room data. The intention was that this data is stored in a database and provided to the model via a corresponding interface. As it turned out, the database was only in the process of planning from both, the technical as well as the data-acquisition perspective.

\footnotetext{
${ }^{16}$ Sample size of eight is arguably not really significant, nevertheless it is worth to discus/ bear the tendency in mind.

${ }^{17}$ If you read so far as to find this footnote and are interested in a chat about this scatter plot and its relevance over a beer, or only in a beer, let me know. I'll be glad to pay the first person a beer or two!
} 
The list of rooms (formally) intended for use as lecture rooms contained 1,274 rooms. Content of this list was solely administrative information such as ID, room code, location, assigned institute, intended usage type (lecture hall, laboratory, etc.) and size. One can imagine that collection and processing of all remaining infrastructure data (primarily available equipment and seating configurations), together with necessary revision, subsequent classification (in machine-readable format) and storing of the data within the (yet to be developed) database is a tedious and time-consuming process. This could either be solved with a dedicated workforce visiting all rooms, collecting, correcting and assessing information and entering it into database, or step-wise by adding these tasks to the routine-tasks already in place. In the latter case information would be gathered and updated room by room when regular tasks are carried out, which would naturally consume quite some time for almost 1,300 rooms. On the other hand a dedicated workforce is expensive - and not affordable in a chronically under-financed institution. To cut a long story short, the project team had to deal with the fact that room data was incomplete - and would remain so for a longer period of time.

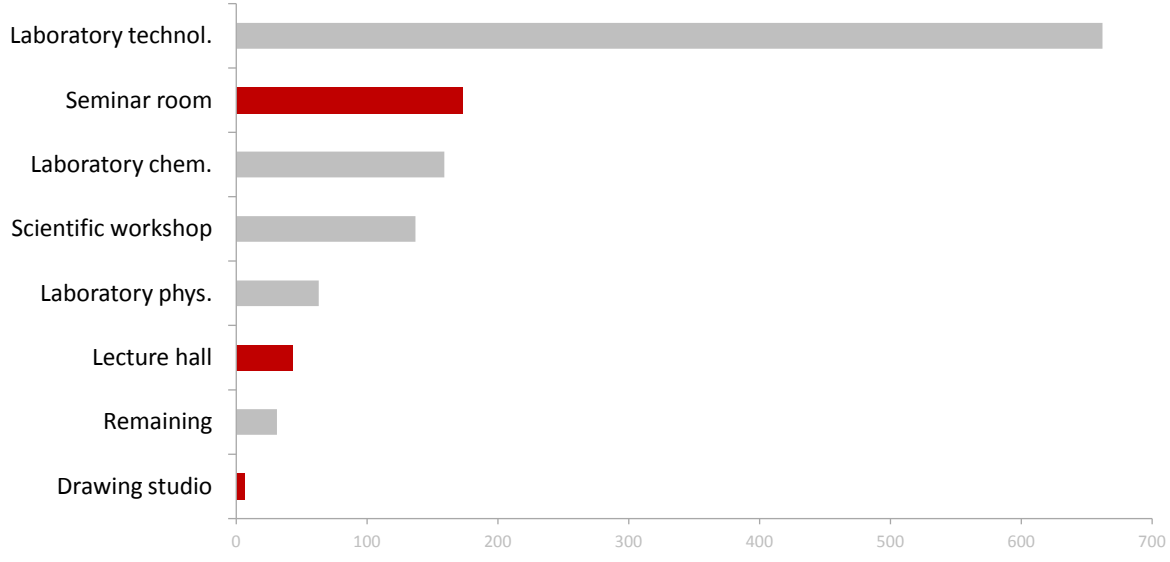

Figure 4.10: Composition of room structure at Vienna University of Technology with number of rooms per category, classical lecture rooms (lecture halls, seminar rooms and drawing studios) are highlighted.

However analysis of the limited information available made it possible to get a better understanding of the system and its dimensions. For example did the distribution of intended room-usage show to be counter-intuitive. One might assume that at a university the majority of rooms for teaching would be lecture halls (including seminar rooms and drawing studios ${ }^{18}$ ). But, as visible in Fig. 4.10, they only account for a fraction (17.4\%, to be precise) of the rooms intended for teaching. The vast majority of teaching rooms are laboratories - all categories (technological, chemistry and physics) combined account for almost $70 \%$ of teaching rooms.

Another data-related problem was the booking-availability of the rooms.

\footnotetext{
${ }^{18}$ Both, seminar rooms and drawing studios, are fairly similar to classical lecture halls. Seminar rooms are usually (much) smaller than lecture halls and without a pitched floor, whereas drawing studios are usually designed with a focus on (natural) lighting.
} 
When creating courses in the respective databases (TUWIS++ and later TISS) via web-interface, lecturers were and are provided only with a fraction of rooms. This number varied over the course of the project (for not communicated reasons) starting with a bit over 200 rooms and currently ${ }^{19}$ holds at 142 - roughly $11 \%$ of the rooms intended for lecture. These 142 rooms again are split in two categories: those with booking approval via TISS (94 rooms, $7 \%$ ) and those where personal approval is necessary $(48,4 \%)$. Which points at the "ownership" issue mentioned in section 4.1.2: organizational units de facto own the rooms assigned to them. In practice the responsibility to administer allocation of these rooms is often contrasted by a practice of arbitrary decisions and assignments - usually to the disadvantage of those not affiliated with the organizational unit in charge.

\section{Booking process}

In order to model room allocation it is necessary that the rules of the underlying processes are transparent. Without the knowledge of these rules a model cannot produce valid results. And it - rightly - will not be accepted. Subsequently the above described practice of room administration is incompatible with the intended model purpose.

To overcome this problem the MoreSpace project team proposed to implement the business process model developed for scenario (2) - real-time space management (see Fig. 4.3). During run-time of the project the team discussed this proposal and other related topics (including the need and benefits of centrally coordinated room-allocation) regularly with the responsible stakeholders: the rectorate, the room-allocation, the GuT and the TISS-development-team. Although a general consensus - that central coordination would improve the situation - was reached, it was impossible to explicitly define a business process. The reasons for this were manifold, including fear of losing control over valuable resources (e.g. "everybody could book and block the special laboratory that we absolutely rely on") as well as a lack of decision-making power (and/or will). To cut a long story short, it was not possible to implement the proposed BPM. Instead an alternative booking process sprung from these discussions.

Unfortunately this process was considerate of as much interests as possible, which eventually lead to an increase of bureaucracy and a chaotic introduction of the process. For example instead of centralizing coordination of lecture rooms (either in one place or for every faculty) a new function was created: every institute was to appoint several "lecture room representatives" who are in charge of administering requests regarding the lecture rooms "owned" by the organizational unit. Further the process allows for reactivation of courses held in the past year, with the benefit of having a higher assignment priority.

This booking process was divided in three phases and introduced towards the end of the MoreSpace project (at the end of the academic year 2009/2010). During the first phase (administrative phase) only the room-allocation group was allowed to delete, modify and add courses and events. The second (reactivation phase) allowed reactivation of lectures from last year and formulation of room-time requests for new courses and events. At the end of this phase the reactivated courses were booked into the system (if not colliding with events from

\footnotetext{
${ }^{19}$ As of June $6^{\text {th }} 2013$.
} 
the administrative phase). During the third (consolidation phase) requests for new events from phase two were either approved or denied. In case of collisions priority was given events from the administrative phase followed by reactivated courses and events.

Apparently the aim of these rules was to preserve course-structures from the past - which proved to work out (more or less). But this arguable advantage was traded for flexibility. Instead of formulating demand for an activity (i.e. required capacity and infrastructure plus time of event) courses or events still request a specific set of room-time and thus severely limit the potential for improvement of allocation.

Introduction of any new process usually induces uncertainties among those affected. So did the introduction of the new booking process. Paired with the process' rules this uncertainty resulted in fear of not receiving the rooms needed for lecture — and subsequently caused a stockpiling behavior among staff.

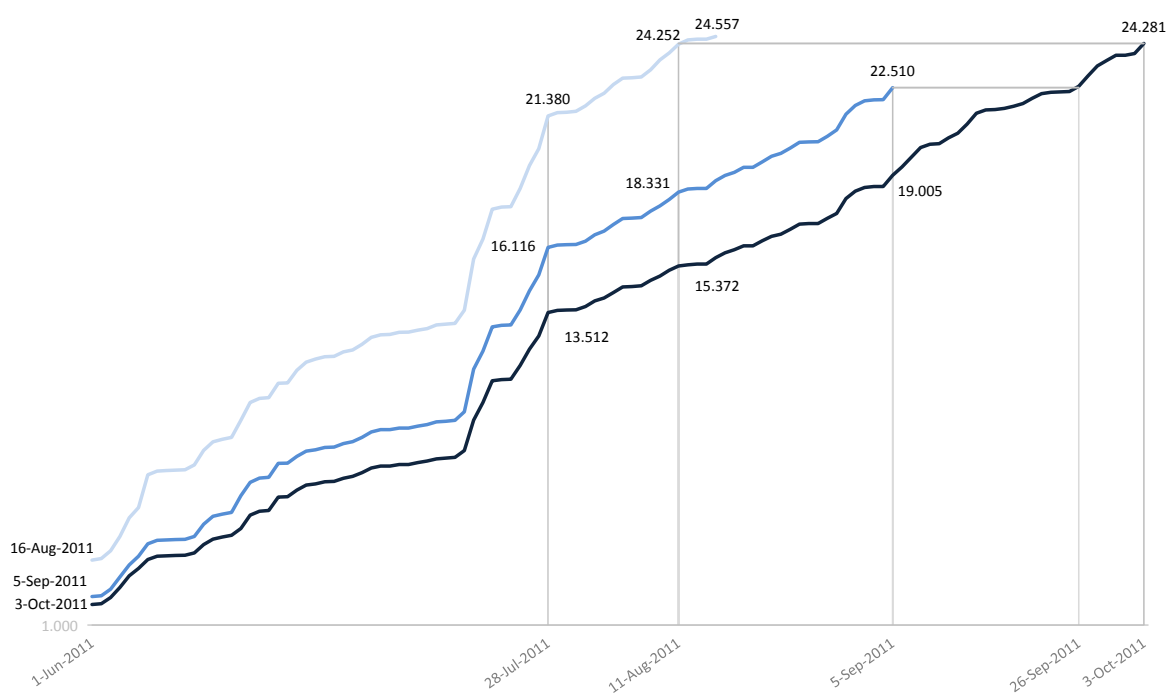

Figure 4.11: Magnitude of lecture-room stockpiling after introduction of (sub-optimal) booking process made visible through repetitive analysis of course-creation-dates.

The magnitude of this effect is made visible in Fig. 4.11. In contrast to the above assessment which was based on courses and course-numbers, in the following events will be used. The reason for this is that at this point of time the transition from TUWIS++ to TISS was already finished. And thus the data came from the TISS (database), which automatically translated created courses into their individual events. The following evaluation is basis on the "created at" entries of all events within the database. The "created at" value of an event describes what its name promises: the date (and time) when the respective event was created within the database ${ }^{20}$.

This data was collected three times: on $16^{\text {th }}$ of August (light blue band in chart), $5^{\text {th }}$ of September (medium blue) and $3^{r d}$ of October 2011 (dark blue). The numbers of created events were accumulated over time. I.e. if on day one

\footnotetext{
${ }^{20}$ This entry is the same for all automatically generated events belonging to a course (created by a user).
} 
there were 50 created events, on day two 12 and on day three 21 , then the line would start from 50 (first day) rise to 62 (second day) and continue to 83 on the third. Each line can thus be interpreted as integral over the number of events within the system.

The interesting part now is the comparison of the temporal developments between the three acquisition dates. On August 16 there were 21,380 events within the database that had been created up to July 28 . Only 20 days later (on September 5) there were only 16,116 events left within the database that had been created up to July 28. This means that (at least) 5,264 (which are $25 \%$ on July 28) of all events within the database were deleted between August 16 and September 5; and between assessment in August and October a whole $7,868(37 \%)$ ! This number is monotonically increasing as time proceeds, as it is possible to delete events ${ }^{21}$ that had been created in the past while newly created ones will carry the current date and thus not influence the sums of past dates. Subsequently the largest discrepancy will be found on the last day at which comparison (of all three data sets) is possible - which is August 16, the day when the first data set was obtained. Here the differences are 5,768 (23\%, Sept. $5,2011)$ and 8,848 (36\%, Oct. 3, 2011). Remark: change of relative figures is not monotonically (increasing) as these depend on the development of the total number and of the difference. Since, in the long run, the system stabilizes (i.e. the accumulated totals of the acquisition dates close in on each other), the total rises faster than the differences and thus the ratio between them reduces.

This is seen when comparing the three final numbers of events within the databases: one realizes that these numbers stay relatively stable - despite the vast amount of deleted events. They differ between assessment in August and September by about $8.3 \%$ and by $1.1 \%$ for assessment in August and October. An obvious assumption which would explain this observation is that of lecturers issuing "blank" requests in order to reserve room-time and to later replace the blocking-events with their regular courses and events. Evidence in support of this theory is found in the number of event-collisions. A collision is defined as an overlapping of room-time specifications of at least one event (i.e. two or more events are taking place at the same time in the same room), where it is regardless whether they overlap only one minute or complete event duration.

The number of collisions has been calculated (based on the event-data from TISS) for the second and third sampling dates. As the total number of events increases from 22.510 (September 5) to 24.281 (October 3) the collisions rise from $1.777(7.89 \%)$ to $1.882(7.75 \%)$. The total figures show a slight increase in collision numbers with a slight decrease of the relative level. It can be argued that the reason for this is: (a) an increase of total collisions as more and more events are populating the database, while (b) at the same time some lecturers are deleting their placeholder events so that the increase is not as strong as that of events. Figure 4.12 shows the change in collision-numbers for rooms with more than ten collisions on $3^{\text {rd }}$ of October - although with no obvious pattern to be detected.

A second - even stronger - piece of evidence for the theory of placeholder events is provided by manual analysis of the occupancy schedules via the TISS' web-based GUI. A screen shot of such a schedule is provided in Fig. 4.13. It

\footnotetext{
${ }^{21}$ It is most likely that the users deleted whole courses, but the effect is the same, as this automatically deleted all of the course's events.
} 


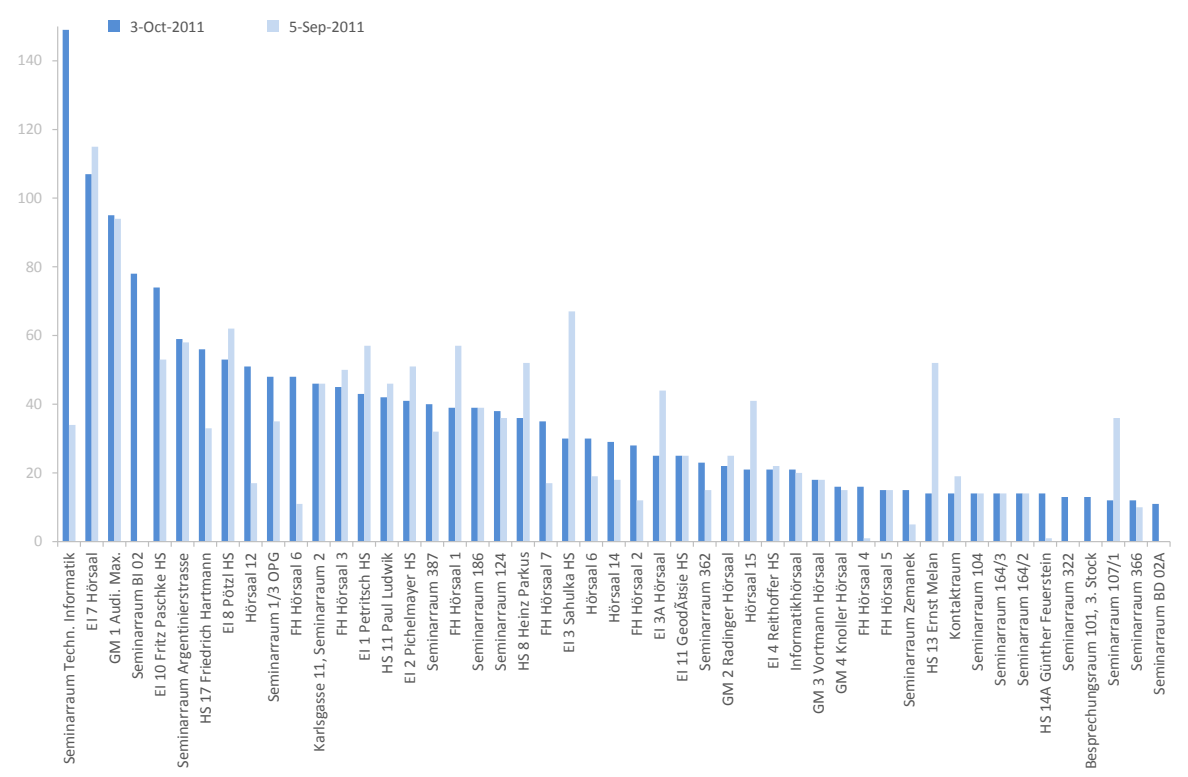

Figure 4.12: Number of colliding events for lecture rooms with more than ten collisions.

shows one weeks plan for the Audi Max, the largest lecture hall of the TU Vienna, which, as shown in Fig. 4.12, has the third highest number of collisions (as of October 3, 2011). The week depicted has a high number of collisions, almost all of which are completely congruent, which is a first indicator for placeholder events. Red events denote exams, blue ones events with course-number and grey ones all remaining events. As visible exams are scheduled in parallel for up to five times (within this screen shot). The reason for this is the fact that organizational units usually handle their written exams batch-wise. I.e. the organizational units schedule all (or several) of their courses' exams for a single date in order to minimize the time necessary for their supervision. Further one can observe that there are no collisions of blue events. Instead those collide with grey events (without course number) - completely congruently and oneby-one $^{22}$. This is another indication for placeholder events, which is backed by comparison of the course-/event-titles and/or -descriptions. For such comparison it is required to understand the content of the titles/descriptions. Since these are not designed for machine-readable interpretation, it is not possible ${ }^{23}$ to automate this analysis. Subsequently not all collisions were checked. Nevertheless the unstructured inspection of collisions' titles and descriptions strongly supported the room-blocking theory.

\section{Impact on Mode of Deployment}

As described in section 3.2.1 requirements for deployment of a model can be classified as structural and data preconditions. The data required to feed the model was, as described above, in a fairly poor state - in terms of quality of

\footnotetext{
${ }^{22}$ Except for one event on Tue, $10 / 11$ at noon.

${ }^{23}$ It might be possible to use or implement advanced content-recognition systems, but this would not have been feasible.
} 


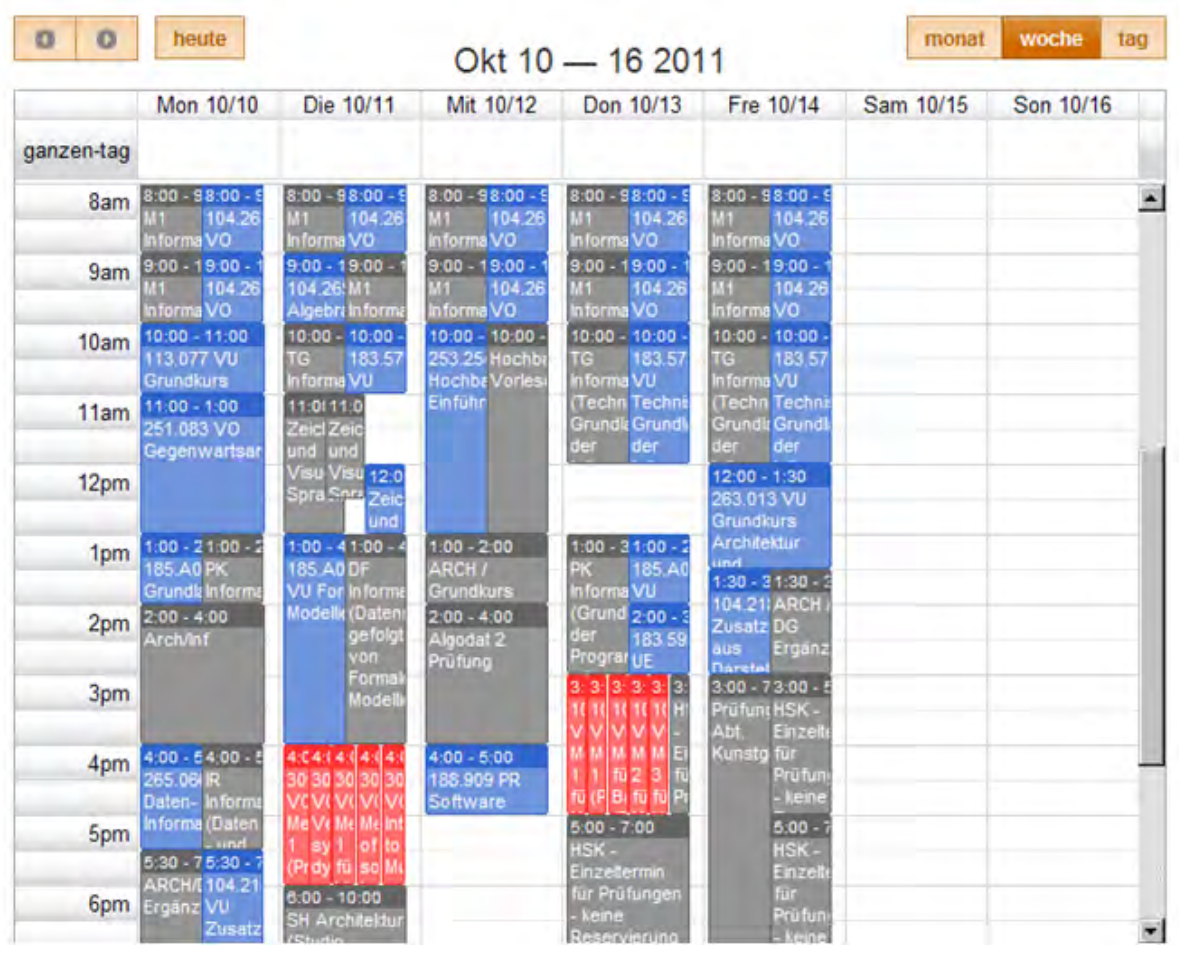

Figure 4.13: Screenshot of Audi Max' occupancy schedule (from TISS' web-GUI) showing intentional - and harmless - event collisions.

the data itself as well as accessibility. At this point it has to be added that accessibility of data improved over the course of the project, as the TISS went online. Via an interface, especially implemented for this purpose, the project team was able to query data via a web-interface which then downloaded onto the requesting client. Nevertheless this data did not include room information. A "room database" was not implemented or set up, as the respective data collection did not finish. Subsequently an important part of the data (classification of rooms) was not available to the model.

On part of the structural conditions the business processes proposed did not get implemented. Instead a compromise solution of all stakeholders involved was formulated and implemented - which did not focus on embedding of the MoreSpace model. Subsequently not only the business processes models required for deployment were not met, but also some of the most important preconditions of these BPM. An example would be the adaption of the TISS-GUI, which can be classified as a mixture of structural and data preconditions. As explained before, the project team issued a list of proposals for adaption of the interface used to create courses/events, in order to guarantee complete data (i.e. guide the user to specify all required information) — which was not implemented.

Most importantly it was not possible to shift the room-allocation focus from a room-time-driven to a demand-driven perspective. I.e. events are still created with explicit room-requests instead of specification of requirements. This focus severely limits the potential for improvement through space management, as it 
is possible to either accept or deny such a request. Only after denying a request would it become possible to propose alternative resources.

In order to assess the impact of these findings, let's look back at the three scenarios which the project targeted at (see section 4.1.1). It can be assumed that testing of system limits or the effect of introduction of new curricula - both scenario (1) - is most likely to happen seldom or with a low frequency. Tests for system limits due to a change in the room structure induced by reconstruction are not every-day tasks, as construction work is usually planned in advance for long(er) periods of time. Hence it is possible to groups such tasks and carry them out in one go. Testing system limits due to changes in the demographic structure or in the educational systems is also going to be necessary only every once in a while. Changing or newly introduced curricula on the other hand are likely to occur more frequently - nevertheless not more often than once per academic year. Thus scenario (1) can be classified as infrequent recurrence or even as a non-recurring case of consulting.

Day-to-day space management operations, defined in scenario (2), are easily classified as frequent recurrence. Depending of the exact specifications, this task is carried out on a daily, weekly or monthly basis, potentially for several times at once in order to come up with a satisfying solution.

The final scenario (3) defines retrospective system analysis. Its primary use is that of obtaining a deeper and better understanding of the system and to evaluate alternative space management strategies with historic data - and thus without the risk of negatively affecting the running system. This scenario is best classified as infrequent recurrence.

As elaborated before, all scenarios require the same input-data structure. Therefore it is irrelevant which of the scenarios is tested for compliance of its preconditions. The important aspect is the intended mode of deployment and its preconditions and requirements, for which the degree of met preconditions, business processes within the peripheral systems and availability of input data needs to be tested. Upon this the deployment matrix (see Table 3.3) is used to evaluate the options and, if necessary, feasible alternatives.

The three scenarios span across the whole range of possible modes of operation, with scenario (2) being the most demanding (frequent recurrence). Both, scenario (1) and (3) are of type infrequent recurrence with scenario (1) even reaching into the non-recurring category, depending on the nature of the assignment. It is visible from the deployment matrix that, if it is possible to deploy a model classified as frequent occurrence, it is also possible to deploy it as infrequently occurring and non-recurring. In same way it is possible to deploy nonrecurring if infrequent recurrence is possible. The modes of operation can thus be seen as proper subsets $\{$ frequent recurrence $\} \subset\{$ infrequent recurrence $\} \subset$ \{non-recurring\}. A model categorized as "frequent recurrence" that can be deployed also qualifies for deployment in all other categories.

In the present case, the preconditions are (by far) not met completely. Hence straight-forward deployment of the model is not possible. For scenario (2), "realtime" space management, the biggest handicap is the absence of an according BPM, followed by the lack of reliable, easy-to-access (i.e. automated access) data. As it turned out to be impossible to implement a BPM addressing the model's needs, it is also not possible to deploy the model for the task intended in scenario (2).

Scenario (3) is, as explained, primarily used for knowledge generation and 
to derive strategies that are to be used for the second scenario. Hence, with scenario (2) becoming impossible, scenario (3) is reduced to its "political" role to argue in favor of such a system and to prove its validity. But as it was impossible to change the BPM at the current point of time, this aspect is not as prominent as initially believed.

Remains scenario (1) - the primary reason for initiation of the project. The model could be used to support decision making regarding the planned reconstruction phase but also to compute the university's system limits. Whether this scenario is to be classified as infrequent recurrence or non-recurring depends on the decision makers. Even though data required by the model is neither of perfect quality nor of such accessibility, it is available (for the most part) and usable. As such some of the model's preconditions are met, and it is likely that the model can be used at least for non-recurring consulting in order to support decision making in the course of reconstruction. Without substantial changes (most importantly improving availability of data) it is a matter of availability of resources whether the model can - and will - be used for periodic testing of system limits and/or changing curricula. It depends on the willingness to afford the personnel necessary for data-preparation.

If a model cannot be deployed as intended and it is not possible to change the business processes, there is one more alternative to aborting the project. It is possible to reformulate the question(s) towards the model respectively to change the project adequately as explained in section 3.2.1. 


\subsection{Modification of Project}

As elaborated in the previous section, model deployment as intended is not possible. At least not for scenario (2) which relies on frequent recurrence mode, which again would require remodeling of the system's business processes. Thus scenario (3) is obsolete in its role as input-provider for the second one. Subsequently scenario (1) is the sole remaining, sensibly deployable one.

This section analyzes the impact of these findings with respect to the project goals. The question arises if the goals (see section 4.1.1) are addressed in a satisfying way and, if not, how to improve the situation. This covers the development of the simulation model itself including workarounds for its deployment (section 4.2.1). As an answer additional analysis- and visualization-tools are created to support decision makers within this specific environment (section 4.2.2).

\subsubsection{Adaption of Deployment}

\section{Implications for Model Development}

When the project team started the feasibility study preceding the MoreSpace project (see section 4, genesis of the project) it chose Enterprise Dynamics (ED) as simulation environment. Due to the good experiences during the feasibility study, especially for implementation of prototypes, ED was kept as the feasibility study made place to the MoreSpace project.

The choice fell on ED for several reasons. Firstly it is a discrete event simulation (DES) environment and thus well suited for modeling of the problem at hand. This was shown in similar preceding projects as described in Wiegand et al. (2006, 2007b). Secondly it is an object-oriented environment, which is extremely beneficial for modeling of agents - which were used as an extension to plain DES. Thirdly ED is a reliable simulation software platform used in various fields of industry. Nevertheless it was necessary to find an adequate mapping of the entity-flow concept onto the given problem of space management.

DES' theoretical basis is the EntityResource- or EntityFlowConcept. Entities are searching their way through processes to the according resources, while this way is controlled or influenced by events. E. g. patients inside an emergency department resemble the entities that are routed through diagnostic and treatment processes to the respective resources (X-ray, doctor diagnosing fracture, etc.). The way of the patients is depending on events (for example a finished diagnosis or freed resources).

Within facility management or more generally within management of the resource "room" — in terms of spaces inside a building - DES have not been utilized until now (to the knowledge of the author). Thus a proper definition or mapping of the DES-concept had to be found: while resources can be identified unequivocally with rooms, several projections are possible for the entities.

Room is generally reserved/booked for a certain demand. This demand can be identified in two ways: as groups of people that want to carry out certain work or tasks or as tasks/work that needs room - and is associated with specific persons. For the present model the first approach has been chosen. 
This required to break down the systems onto the individual users, as the groups - which carry out work (i.e. courses or events) within the rooms - are changing. Individuals can be "members" of several groups, which again can be "acting" at the same time. In this case the respective individual can of course not participate in both groups and consequently - in the worst case scenario - a group could represent a null set and thus not require space at all!

From this it follows, that although the entities of the DESconcept are represented by the groups, individuals are playing a key-role (at TU Vienna roughly 20.000 individual students need to be considered). These individuals are implemented as a combination of DES-atom and agent-based (AB) element. In such a way individual controller logic (their "schedule") can be assigned to each one of them. Thus these agents or atoms are acting in response to events (lectures) but also goal-oriented as they decide how to proceed when conflicts arise - a feature that adds much to the dynamic system behavior as well as it brings it closer to reality.

The next obstacle that needed to be overcome was the adaption of the model to a changing system. The implementation of DES models usually takes place using simulation environments (Enterprise Dynamics in the present case) that offer model-libraries and support drag-and-drop model creation, which offers a certain amount of flexibility and ease of use. Still it becomes hard to manually create a model consisting of several thousand entities (events taking place) and several hundred resources (rooms). Even more as those are subject to change - e.g. changing building plans during construction, blocked rooms due to repair or maintenance, changing curricula, etc. The complexity of the necessary input-data is displayed by the ERDs in figs. 4.5-4.7.

To conquer this obstacle a module for database-driven model generation has been developed (see Tauböck, 2010, which also includes a detailed description of the ED model). It enables the model to be created automatically and fast out of (room-)databases. Subsequently the model becomes highly flexible and can represent virtually any room structure - given the necessary database. Another advantage of this structure is the ability to also modify rooms: if necessary two adjacent rooms (equipped with appropriate infrastructure) can be combined to create a bigger one and vice versa.

- Quoted from Emrich et al. (2010).

Initially Microsoft Excel was used to provide this data. But it soon became obvious that the spreadsheet program limited the simulation model. Firstly the access time for read-/write-operations between ED and Excel was slow. Secondly a database was better suited to administer the (input) data. Subsequently Excel was dropped for in favor of Microsoft Access. The choice was an arbitrary one as SQL could have been used as well.

Cellular automata were used to model walking times between (lecture) rooms. CA are especially suitable for this as their structure is based on a spatial grid that can be easily translated into any 
spatial context - though a problem lies within the individualism of the entities that are to be simulated. Again an agent-based extension was used to overcome this shortcoming ${ }^{24}$. With this approach each individual can be clearly identified and thus passed forth and back between the DES-model and the CA-model in order to compute its walking times (see Bruckner, 2009, for a detailed description of the model).

In order to increase the computational efficiency the university campus is not depicted by a single CA - which would inevitably consist of many "white areas" which do not need modeling. Instead the buildings are broken down to hallways and corridors which are mapped onto individual CA. This does not only result in slender automata but also allows for parallelization of their computation.

The CA-model developed is connected to the ED-model via a TCP/IP interface, which is used to send individuals from one model to the other. As such a person traveling between two lecture rooms is sent to the CA-model as departs from room one. Within the CAmodel the travel is simulated and, as soon as the individual reaches its destination, the agent is again handed over to the ED-model.

As real-time simulation of walking times is consuming a high amount of processing power when utilized for the whole university campus, the CA is used in order to compute sound approximations for walking times as function of the number of people in the hallways. This correlation is calculated for each two lecture rooms using the Monte Carlo method and stored in lookup-tables. Figure 4.14 shows such a correlation of walking times and the number of simultaneously moving agents for two lecture rooms (HS11 and $\mathrm{HS}^{25}$ ). The number of persons within the building is found on the $\mathrm{X}$-axis and (minimum, maximum and average) travel times in seconds are plotted on the Y-axis. Depending on the required precision of walking times, it is then possible to either couple the CA-model and simulate highprecision values or to use the lookup tables (with average values) for increased performance.

- Quoted from Emrich et al. (2010); reformulated and

expanded.

In the course of the project the ED-model developed was regarded as "in the making". Several prototypes have been developed and refined in order to react on changes or to include additional features. This was, as previously mentioned, also one of the reasons for sticking with the ED environment. According to the project plan the model's final functionality was re-implemented in an independent at the end of the project. The reasoning behind this is that in such a way the model is independent of any simulation environment and can thus be distributed without the problem of licensing (regardless whether it is used by the MoreSpace team for consulting purposes, sold or given away for free). Further, when building a model from scratch one avoids overheads that

\footnotetext{
${ }^{24}$ Reference is given to Emrich (2007) and Emrich et al. (2008) for further information on the combination of $\mathrm{CA}$ and $\mathrm{AB}$-methods.

${ }^{25} \mathrm{HS}$ abbreviates Hörsaal, which is lecture hall in German.
} 


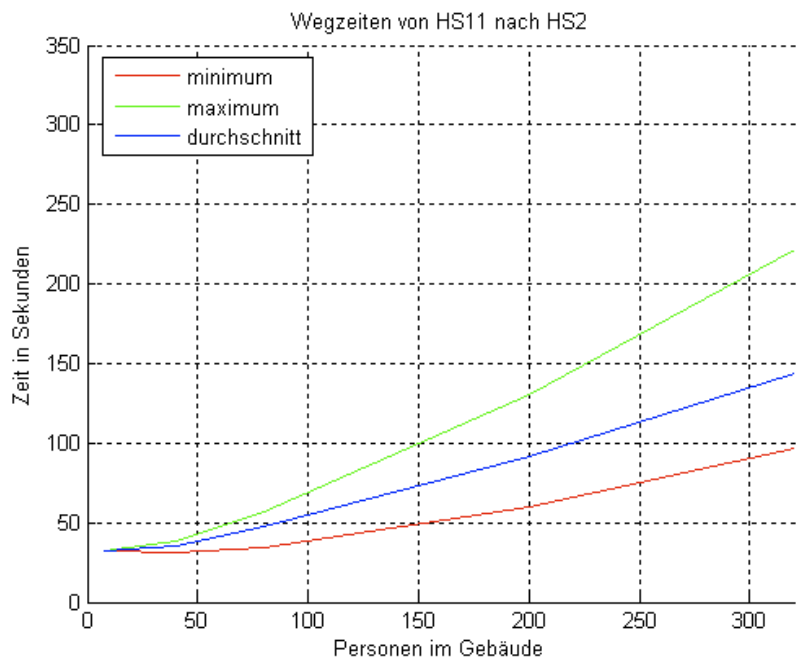

Figure 4.14: Monte Carlo simulation of walking times (y-axis, time in seconds) vs. number of moving agents (x-axis) with average time (blue), maximal (green) and minimal (red) time consumed.

many programming- and simulation-environments produce. Hence the resulting software is more efficient in terms of runtime and necessary resources.

As explained in section 4.1.3, one of the things experienced was that business processes and database-interfaces are not always available in the required form - regardless of the intentions at project start. Subsequently it was the goal to incorporate the highest possible flexibility into the final model. For this reason a program was developed which is consists of a core-framework and plug-ins for components that are frequently subject to change. This includes the import- and export-interfaces, the booking algorithms and parts of the postprocessing capabilities. Usually these are user-specific, as the databases (if existent) will vary in software and structure, while the booking requirements strongly depend on the business cases and processes of the user. The core of the model - the framework - was implemented in JAVA, while JavaScript was used as language for the plug-ins. JAVA was chosen due to its free license, objectorientation (to implement $\mathrm{AB}$ methods) and great publicly available libraries (fast implementation). JavaScript was chosen with regard to the ability of users who want or need to modify interfaces or booking routines themselves. With JavaScript being a "simpler" programming language than JAVA, the idea was to empower users with limited programming experience to modify and adapt the necessary scripts.

\section{Deployment - Scenario (1)}

The project's initial main goal was to answer how closing of lecture halls (especially of the largest ones) for renovation would affect daily business. With a basically deployable scenario (1) this question can still be answered, although it is necessary to find workarounds to overcome data gaps. In the case of the TU Vienna the biggest question was, what will happen if the Audi Max (the universities by far largest lecture hall) is being closed for retrofitting. The assumption 
was that additional space will have to be rented in order to accommodate the events originally located in the Audi Max. The estimated costs for renting of these external facilities were calculated as a quarter million euro over the whole construction period.

The BPM for scenario (1) - see Fig. 4.2 - is driven by the GuT, who is in charge of retrofitting as well as of provision of adequate lecture space. The GuT was also to provide simulation input on the rooms (availability, status, etc.). The remaining data (student numbers, courses/events) was obtained from the TISS. Due to planning requirements (organization of construction work, contracting of companies, renting lecture space, etc.) room blocking decisions have to be worked out well in advance. Thus the input data for simulation of the impact can only be of historic nature, simulation results thus only approximations.

In order to simulate the effect of the blocked Audi Max it was necessary to redistribute all events on the remaining rooms. This required to take the event data from the TISS without rooms that they eventually had booked. In doing so all information regarding the events' room requirements (in terms of infrastructure) was lost, as the booking process in place is room-time driven. Since no classification of lecture rooms existed either, the lack of events' infrastructurerequirements did not make things worse.

To cope with this fact all lecture rooms available for booking via TISS were put into one category. Event data was only considered of those courses/events that had (prior to removing this information) been assigned to one of the lecture rooms that were offered through the TISS. The assumption was that with this proceeding the considered events and available lecture rooms were roughly balanced. Another approximation was necessary to assign schedules to the (individually modeled) students. This distribution was done based on the number of exams which were multiplies with a correction value (which was subject to expert knowledge).

These approximations and workarounds were also possible as it was clear that the event-room assignment generated by the simulation would not be implemented. The purpose was not the generation of an occupancy schedule but an assessment of the lecture rooms' potential, which was to serve as decision support.

The Audi Max had a capacity of 643 , while the second largest lecture hall of TU Vienna ${ }^{26}$ only fits 434 . Thus a solution had to be found for all events with a higher required capacity than the EI 7 provides. For such events the simulation was allowed to merge two lecture halls (the "Kuppelsaal" 27 and the "Prechtl-Saal" ${ }^{28}$ ). These lecture halls were chosen for the following reasons:

- They are spatially close to another located at the same staircase separated only by 3 floors and accessible for handicapped.

- They offer a combined capacity of up to 680 with the "Prechtl-Saal" being equipped with partition walls, allowing it to host 100 people in the smaller, 200 in the larger part and 330 if combined.

\footnotetext{
${ }^{26}$ The HS EI 7 where HS stands for Hörsaal and EI for Electrotechnisches Institut, thus lecture hall "Electrotechnical institute, 7".

${ }^{27}$ Literally cupola hall.

${ }^{28}$ Named after Johann Joseph von Prechtl, the founder of the "Polytechnic Institute" , the predecessor of today's Vienna University of Technology.
} 


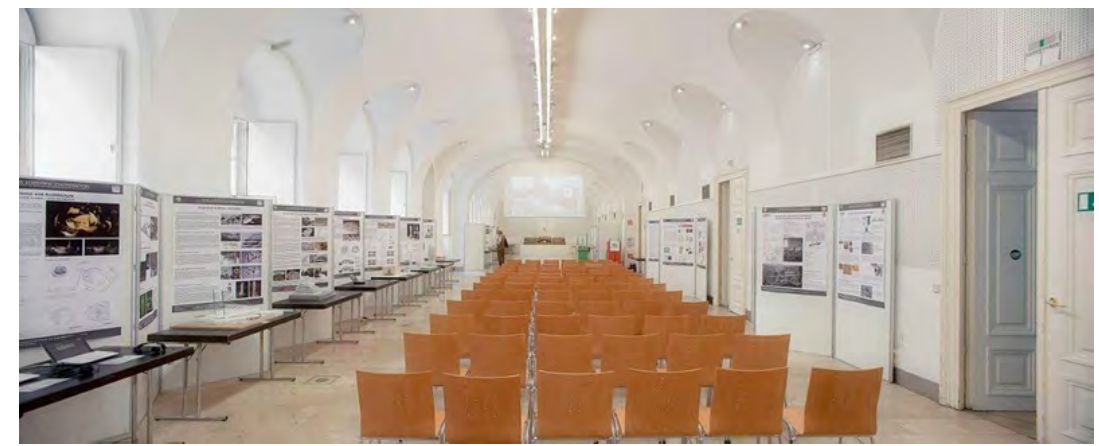

(a) 'Prechtl-Saal"

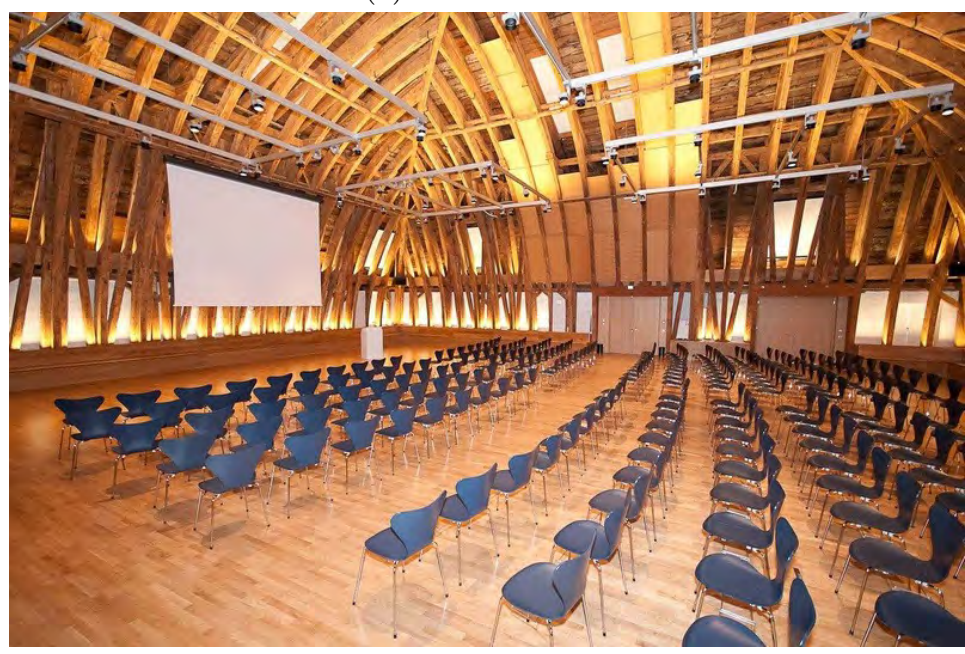

(b) "Kuppelsaal"

Figure 4.15: The "Prechtl-Saal" and "Kuppelsaal", chosen to serve as replacement for the Audi Max during its retrofit.

Subsequently this would even allow to fit the capacity to the event in steps of $350,450,550$ and 680 .

- Most importantly: both lecture halls (see Fig. 4.15) were in use (almost exclusively) for representative and external events - resulting in a fairly low utilization rate.

\section{Conclusion}

Simulation results for blocking of the Audi Max, together with static utilization analysis (which are elaborated in section 4.2.2), showed that Vienna University of Technology has sufficient resources to cope with the blocking. It was also possible to show, that the creation of a virtually merged room - the combination of the Kuppelsaal and Prechtl-Saal - is capable of satisfying all room-request with high capacity demand. Subsequently renovation of the Audi Max was scheduled as planned - although without having to rent additional space for lecture. Instead the Kuppel- and Prechtl-Saal were equipped with audio- and 
video-equipment allowing for live-streaming including feedback from one hall to the other. With this decision the university was able to save an estimated 250,000 euro otherwise spent only on space used for compensation of the Audi $\operatorname{Max}^{29}$.

In the course of data assessment the team consulted the deans of several faculties in order to improve the data available. In these talks the deans expressed a great interest in better understanding of the room-allocation process and especially in having a better overview of the actual room utilization. The complete lack of respective information was mentioned as a major problem for management and coordination.

It is this understanding that should be facilitated by the set of questions directed towards scenario (3). Its output can be subsumed as "gaining deeper system understanding" - put aside generation of input for scenario (2). This system understanding could be generated with the help of the model (relying on workarounds to provide the data necessary). A second possibility is to come up with alternative approaches to improve system understanding. With scenario (2) being impossible to deploy there is no need to generate input for it either. Hence it does not make sense to force using a resource intensive approach (i.e. feeding the model with data obtained by costly workarounds).

\subsubsection{Visualizing Room Utilization}

Since deployment of scenario (3) was not feasible in the present case the decision was made to pursue alternative strategies. The goal was to use (static) data analysis and information visualization to extract as much information as possible in order to compensate the shortfall of simulating scenario (3). The questions this scenario should have answered (see section 4.1.1) require simulation to evaluate the effects of changes (e.g. of space management strategies or of room structure). These cannot be answered by analysis of static data. Instead a general image of the system status can be drawn, with a focus on bottlenecks.

\section{Lecture Room Utilization at TU Vienna}

During the course of the project it was found that many stakeholders "felt" deficits in the system (event-room allocation) or room-utilization, but these individual impressions arose without facts. Moreover there was a general absence of figures or data that would characterize the systems (overall) state (in terms of space utilization)! This finding is in hard contrast to the finding of HEFCE (1999, p. 43) which states that "for efficient space management to be achieved, the universities will require full information about their existing space, its capacity, its use, its level of space utilisation and costs which are attached to that space. It is only by having an awareness of the full extent and composition of all the existing building stock that improvements in efficiency can be properly planned and implemented." Hence introduction of methods that would allow a first assessment of the system's state would alone pose a great improvement of the situation.

For this reason the project team carried out an analysis based upon the event-to-room assignment data available. As complete data was only available for lectures that were being held in the rooms listed in the TISS, the data only

\footnotetext{
${ }^{29}$ Estimated by GuT.
} 
represented a part of the whole system. Then again the lecture rooms listed in TISS appeared as the most problematic ones in terms of conflicting requests and demands by lecturers and organizational units.

Without reliable numbers of visitors for the events it was not possible to compute rooms' occupancy and subsequently also not their utilization as defined in section 4.1.1, instead the frequency usage was taken for primary analysis. The diagram in Fig. 4.16 shows the amount of requested room-hours in contrast to the total available room-hours during core-time (light gray). With respect to the apparent lack of space a core-time of 10 hours per lecture room and lecture day was assumed. I.e. 10 hours for every days of the semester which is neither a public holiday - including vacations - nor a Saturday or Sunday. This core time would allow for use of the lecture room from 8:00 to 18:00. The data used for the evaluation was assessed on August 16 and October 3, 2011.

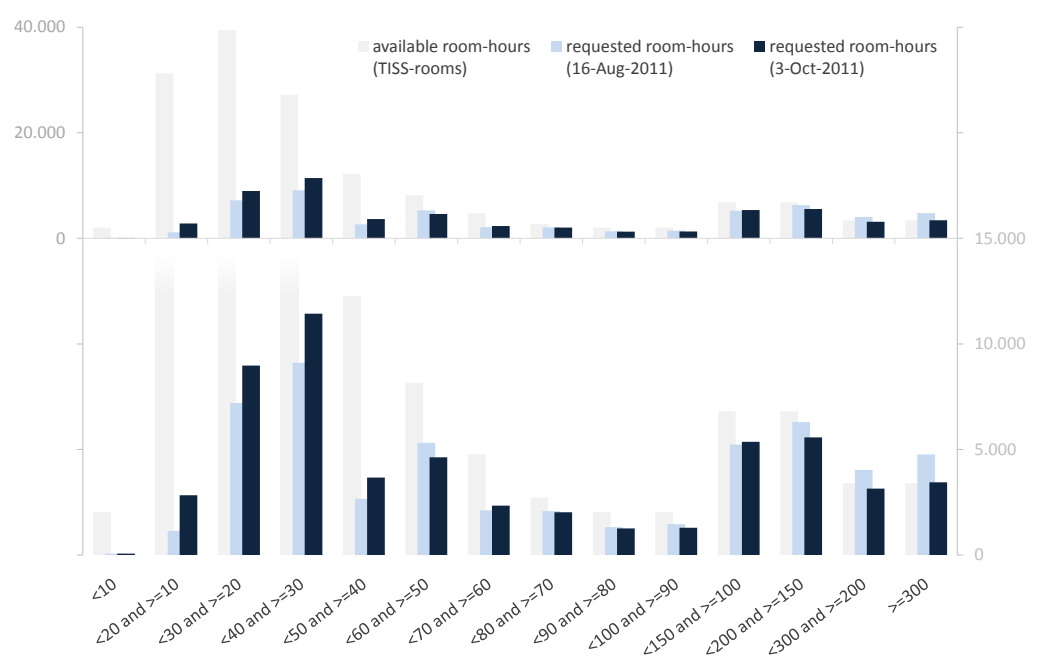

Figure 4.16: Frequency usage of lecture rooms by capacity-classes, as requested on August 16 (light blue) and October 3, 2012 (dark blue). Light gray bars: total available room-hours in core-time ( $13 \mathrm{~h}$ per room and lecture day).

The graph exposes an extremely low frequency usage of lecture rooms with a capacity of up to 50 and a very high one of the very large ones (capacity $\geq 200$ ). For better readability the upper part of the chart is giving an overview of the whole data (with peaks of up to 40.000 room-hours), while the lower part focuses on the amount of requested room-time. It is visible that all but the two largest categories provide enough room-time to easily fit all evens with room-time requests - under the assumption of $13 \mathrm{~h}$ core time and reasonable scheduling (i.e. no collisions, no unusable gaps between events, etc.). And even the two instance of requests exceeding available room-time (capacities " $\geq 200$ and $<300$ " and " $\geq 300 ")$ corrected itself by the second data sampling.

With the insight from section 4.1.3, which suggests that a substantial amount 
of the requests within the system are placeholder events, one can conclude that the current room structure is providing sufficient resources for all planned events - including reserves. This is assumption is backed by comparing events' roomtime request and their calculative course volume (CCV), which can be seen in Fig. 4.17. The comparison shows that lectures more than double $(231 \%)$ the number of room-hours was requested than one would expect from the CCV (data assessed on October 3, 2011). For tutorials the ratio is even more extreme, with the difference between expected and requested lecture space being almost factor six (595\%). Though it has to be noted that for tutorials the CCV has not been corrected to compensate for multiple groups. Depending on the course tutorials are split into up to several groups in order to obtain a feasible groupsize. Nevertheless, the discrepancy between CCV and requested room-hours is large enough to support the initial assumption of overbooking.

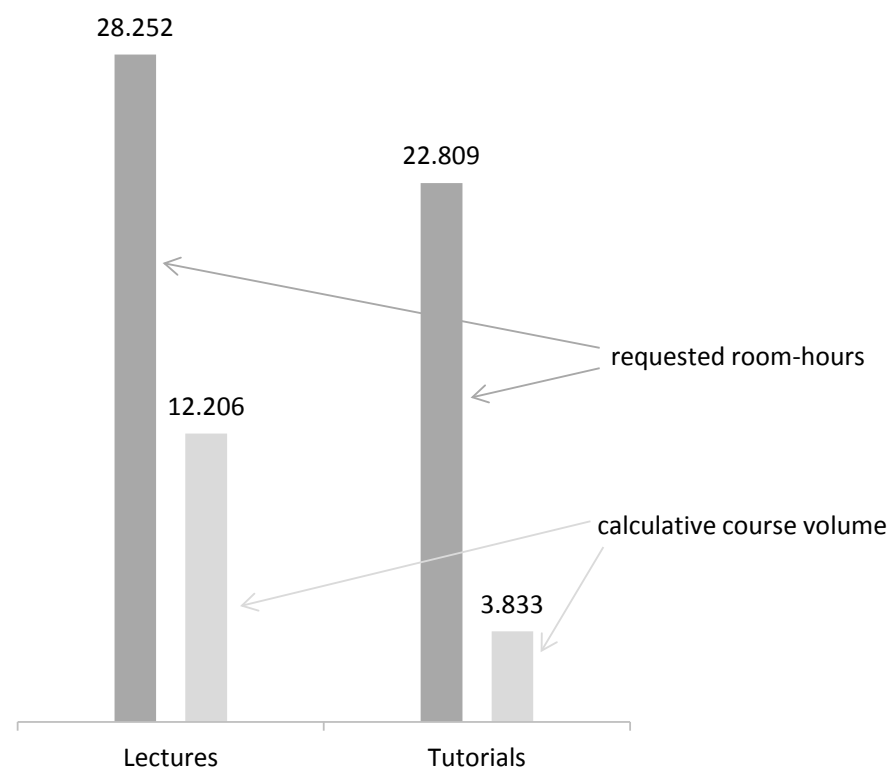

Figure 4.17: ATTENTION: Find better approach to labeling bars than by THESE arrows. Expressed (dark bars) vs. expected (light bars) lecture room demand of lectures and tutorials; expressed demand in room-hours, expected in calculated course volume (CCV).

TISS-data was the only available source of information on actual room requests. While the TISS covers all courses offered, it only covers a relatively small set of the university's lecture rooms. Subsequently room requests are only registered if a course requests one of the TISS-lecture rooms. All other courses' room demand remains unknown. The implications of this are shown by Table 4.3. It shows the number of courses and the ratio of courses without expressed room demand ${ }^{30}$ for the eight most common course-types ${ }^{31}$. It is obvious that lectures and lectures with tutorial have a much lower rate of courses without room request than the remaining types. Tutorials (47\%) are also far

\footnotetext{
${ }^{30}$ I.e. no demand for one of the TISS-lecture rooms has been formulated.

${ }^{31}$ The next largest category ("excursion") only has 28 courses.
} 
Table 4.3: Courses without a TISS room-request (relative figures) and total numbers by course-type.

\begin{tabular}{lcc} 
Course type & without room request (\%) & total number \\
\hline Exclusive tutorial & $96 \%$ & 320 \\
Research project & $77 \%$ & 146 \\
Laboratory tutorial & $75 \%$ & 170 \\
Seminar & $73 \%$ & 639 \\
Academic internship & $72 \%$ & 175 \\
Tutorial & $47 \%$ & 245 \\
Lecture with tutorial & $27 \%$ & 397 \\
Lecture & $23 \%$ & 714 \\
\hline
\end{tabular}

apart from the remaining five entries, but already with much less requests. The reason for this can be found in the nature of lectures, which usually require lecture halls (or at least large lecture rooms), and these are all within the TISS-set of rooms. Tutorials are usually for smaller groups of students, thus most of them do not rely on large lecture rooms. Further it can be assumed that laboratory tutorials will require laboratories - which are not covered by the TISS - and that exclusive tutorials are for a fistful of students and thus often take place in small meeting rooms. Research projects and academic internships often require a single coordination event and afterwards no explicit room any more, as the students are having individual meetings with their coordinator(s), e.g. taking place in the coordinators office. The last remaining category is that of seminars. These might take place either in small rooms not covered by TISS or they do take place in TISS-rooms, but rooms which are "owned" by the organizing units. In the latter case it is possible that the events are scheduled during a coordination meeting at the beginning of the course, but without transferring of this information into the TISS.

Summing up the information of this (brief) static utilization analysis, it can be said with a sufficient degree of certainty that for the courses and rooms covered by the TISS the system holds a fair amount of reserves. At the same time it is apparent that this set of courses and rooms is only a fraction of the whole system - although the problematic one. With respect to the discrepancy between $\mathrm{CCV}$ and requested room-time the call for a re-formulation of the booking process (key-words: transparent and centralized) is once more backed.

\section{Visualization Outline}

In the following a more general approach to visualization of space utilization (than the above specific analysis for Vienna University of Technology) is introduced. For result-visualization three types of data are interesting and potentially significant:

1. Event-data information directly associated to events. 
2. Room-data

information directly derived from rooms.

3. User-data

information obtained through (optional) user-simulation.

Reasoning for this order is as follows: The center of attention is the actual "utilization" of rooms, however this may be defined. In this respect rooms are important, but are taking on a passive role - they are either available or not. When taking a look at the coupling of the three entities, it becomes evident that events are the pivotal point: They can ...

- be without assigned or requested room,

- have an allocated room and ...

- have data regarding user-behavior (from user-simulation) or

- be without user-related information.

Thus it is possible to describe events by the Venn diagram depicted in Fig. 4.18, where the set of all events has the two (proper) subsets of events with room and events with room and user-information.

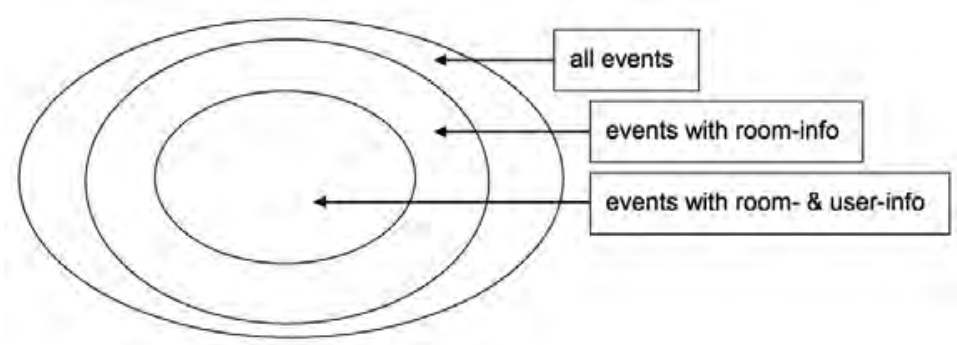

Figure 4.18: Venn diagram of the three (sub)sets of events.

For visualization purposes this Venn diagram is to be inverted. It is possible to carry out analysis for all events (without room- and user-information), followed by analysis for those events with room-information (with and without user-information) and finally for events that have user-information as well (which requires an assigned room).

Remark: In the following user-information is not being used for evaluation in as far that users or groups of users are being focused upon (e.g. which events are frequented most by people with beard?). User-information is used solely for assessment of occupancy, i.e. as possibility to filter used rooms (e.g. rooms with occupancy of less than $30 \%$ ).

Evaluation and visualization requires quantification of data. Quantification again is ambiguous. The possibilities depend on the data available as well as on the goal of the representation. The following quantities can be used as labels for the axes of visualization/diagrams.

Quantification of Events - Events can be measured in terms of their number or their duration, which leads to following sub-groups:

- Number of events 
- absolute figures

- relative figures

- Event duration

- absolute duration

- relative (to the duration of "other" events) e.g. how large is the fraction of events of type "lecture" in relation to the total event duration?

- relative to the amount of room-time e.g. how much of the available room-time ${ }^{32}$ of lecture hall 1 is used by events (within lecture hall 1 )?

Quantification of Rooms - Rooms can only be counted, i.e. quantified only by their number. Although it is possible to calculate the amount of room-hours which they provide, but this is directly proportional to the number of rooms (due to the constant multiplicative factor "time"). Thus measurement is possible of:

- Number of rooms

- absolute figures e.g. how many rooms are there?

- relative figures e.g. how large is the fraction of lecture halls compared to total lecture rooms?

Quantification of Users - As previously explained, user-information is not used for direct evaluation. Instead it is introduced into the measurement of occupancy (used spaces' proportion of room's capacity - at a given time-slice).

From the perspective of events it is now possible to use their attributes in order to group, filter and sort and then evaluate and visualize them. According to the (sub)set of events one focuses at there are more or less attributes to be used (see Fig. 4.18). Thus following evaluations are possible:

1. All events

- classification by start of event which can be grouped by ...

- day of the week

- time (hours or minutes)

- month

- calendar day

- lecture day /weekend, vacation or holiday

- core-time and remaining time

- combinations of the above

- classification by end of event although theoretically possible it is of limited practicality; grouping as previous

\footnotetext{
${ }^{32}$ Remark: Available room-time needs to be defined! It can either be defined as a given core-time (e.g. 08:00-17:00) or as the maximum available time (i.e. 24 hours per day, 7 times per week, 365 or 366 days per year).
} 
- classification by length of event

- classification by required (minimal) capacity

- classification by "priority"

- organizational unit

- type of event

- required type of room

- "movable" events ${ }^{33}$

- "fixed" events ${ }^{34}$

- "priority" of events ${ }^{35}$

- combinations of the above

2. Events with room-information these additional attributes are available only for events with assigned $\operatorname{room}(\mathrm{s})$.

- events with vs. those without assigned room

- comparison of demand (event duration) and available resources (roomtime)

- by room (room $1, \ldots$, room $n$ )

- classification by location of room (e.g. building)

- by assigned room-type ${ }^{36}$

- by assigned capacity ${ }^{37}$

- by "owning" organizational unit of assigned room $^{38}$

- foreign events in "owned" rooms

- own events in "own" rooms

- own events in "foreign" rooms

3. Events with room- and user-information

- occupancy of events

In an analogous way it is possible to evaluate and visualize from a roomcentered perspective. In this case the attributes and respective evaluations are:

1. All rooms

\footnotetext{
${ }^{33}$ Movable events are a class of events which the MoreSpace model is allowed to reschedule within a maximum temporal distance - specified via a simulation parameter - of the intended starting point (e.g. moved front or back for up to $t$ minutes).

${ }^{34}$ Fixed events are a class which has their room assigned already prior to simulation (e.g. high priority events organized by the rectorate).

${ }^{35}$ MoreSpace offers the possibility to prioritize events.

${ }^{36}$ Remark: The room-type requested or booked has to comply with event-requirements. Thus this can be used as comparison only; basic evaluation can be made with eventinformation alone.

${ }^{37}$ Remark, as before: Since rooms' capacity has to be greater or equal to event's demand, it can be used only for comparison; basic evaluation can be made with event-information alone.

${ }^{38}$ Remark: The organizational unit offering an event does not necessarily have to be the one "owning" the room in which it is taking place.
} 
- spatial (or geographic) distribution of rooms

- capacity of rooms potentially relative to total capacity (of a building)

- by room category

- by building

- by "owning" organizational unit

2. Rooms with event-information

- classification by events assigned to room (number/duration) e.g. in how many rooms are taking place 0-99 events, 100-199 events, etc.? Possible incorporation of capacity, e.g. total capacity of rooms with 0-99 events, ...

- usable/not usable rooms (special case with " 0 " and "> 0 events")

- classification by requested date e.g. how many rooms (or which capacities) are being requested/ booked between 0:00 and 0:59, 1:00 and 1:59, etc.?

- classification by requesting event-type

- classification by requesting organizational unit

3. Rooms with event- and user-information allows for statements regarding

- booked but not used lecture rooms

- classification by occupancy

For the above listed evaluations a set of five "standard-visualizations" has been defined, of which three are distinct (line chart, bar chart, heat map) and the remaining two modifications ("step function" and histogram). These charts allow to analyze one or multiple filters as well as one or multiple experiments and are defined as:

- Line chart $\mathrm{x}$-axis: any of the previously defined values ${ }^{39}$ y-axis: any of the previously defined z-axis ${ }^{40}$ : optionally usable for comparison (e.g. multiple experiments) target value on: $\mathrm{y}$-axis

- Step function $\mathrm{x}$-axis: discrete values (any of the previously defined, sufficiently connected)

y-axis: any of the previously defined

z-axis: optionally usable for comparison (e.g. multiple experiments) target value on: $\mathrm{y}$-axis

\footnotetext{
${ }^{39}$ As long as they are "sufficiently connected" or ordered; usually time series'.

${ }^{40}$ For all charts but the heat map the z-axis denotes multiple instances of the chart displayed next to each other (e.g. the $2^{\text {nd }}, 3^{r d}, \ldots$, line in a line chart).
} 
- Bar chart

$\mathrm{x}$-axis: limited number of discrete attributes

y-axis: any of the previously defined

z-axis: optionally usable for comparison (e.g. multiple experiments)

target value on: $y$-axis

- Histogram

$\mathrm{x}$-axis: discrete groupings of attributes

y-axis: any of the previously defined

z-axis: none

target value on: $y$-axis

- Heat map

$\mathrm{x}$-axis: discrete values or discrete groupings of attributes

y-axis: discrete values or discrete groupings of attributes

Z-axis: any relative values, color coded

target value on: $\mathrm{z}$-axis

Visualization and comparison of data (static system data or simulation results) can be conducted on three levels:

1. General comparison of multiple data sets (of static system data or simulation results).

2. Comparison of multiple data sets' details.

3. Analysis of details of one data set.

In addition to the chosen chart the sensibility to visualize given information also depends on the chosen level. For example does it seem reasonable to start analysis with "macroscopic" evaluations or comparisons (detail level 1), such as looking at the number of unsatisfied booking requests. Then, in a next step one might want to look into the details of interesting experiment and compare those against each other. Finally, the most detailed analyses (level 3) are limited to evaluation of a single data set.

While this proceeding shall serve as a guideline, it has to be noted that it is extremely difficult and complex to depict all possible combinations. Hence limits of such predefined methods have to be accepted.

\section{Decision Support Tools}

The above visualization outline has been used to develop the visualization features of the MoreSpace tool. For this a procedure was defined which step-wise narrows down the users possibilities, guiding her towards a sensible result. In a first step the user chooses the diagram type, which automatically limits the possible choices for the x-axis (e.g. choosing a line chart implies a time-scale on the $\mathrm{x}$-axis). Further the combination of chart-type and $\mathrm{x}$-axis values limit the possible choices for the $\mathrm{y}$-axis. The product of this procedure is a treestructure in which the trunk represents the diagram type and the trees' leafs the individual visualizations.

With respect to the encountered problems (e.g. impossible deployment for scenario (3) and poor data-quality and -availability) it came natural do use this visualization outline to create additional tools in order to facilitate decision 
making. Here the focus was set on fast and easy assessment and the possibility to use the instrument even with limited data - in terms of quality as well as of quantity. An additional reason for this (besides the problems encountered) was the possibility to use such an instrument for awareness raising.

In order to keep the entry threshold low enough but the potential insights meaningful enough a web-based tool - with two levels of detail - has been developed. The light-weight version is referred to as Static Analysis Tool (STAN) "Basic" and the more detailed one as STAN "Advanced". For the Basic one does neither need exact real world data nor needs one to understand the implications of (a more efficient) room utilization.

The tool (web-GUI depicted in Fig. 4.19) requires to specify the number of rooms within the building(s) and - optionally - their average size. Next the user is asked to provide a period of time that she wants to examine (i.e. start and end date) and to specify the institutions (or companies) core working times. This is followed by input of required room-hours by events, which can either be entered directly (i.e. $t$ hours of necessary room-time) or via a combination of estimated event-number and average event-duration. Finally the user has the option of adding the estimated amount of external events within and outside of the core time. After each entry the result-charts are updated, so that the user immediately gets a feeling for changes. If she is OK with the entered information it is possible to save it as the user's master data.

The tool now shows the room-hours available within the users system during core time. It also compares them to "ecological operation time" ${ }^{41}$, to a $24 / 7$ benchmark and to the room-time necessary to allocate the user's events' demand for room-time (top left bar-chart in figure).

The top right chart displays the calculative number of rooms which is required to host all events with specified core-time. Again with comparison to the number of rooms needed if ecological operation time or 24/7-utilization would be used within the system.

The lower left chart shows frequency usage of the system's rooms relative to available room-time during core time, during ecological operation time and with 24/7-utilization.

Finally - if the average room size has been specified by the user - the lower right chart displays annual life cycle costs (LCC). Again LCC are compared for (a) the number of rooms in the current system and the minimum number of rooms that would be needed to fit all events into (b) core time, into (c) ecological operation time and into (d) 24/7-utilization. I.e. the LCC are calculate for the use-specified number of rooms and for the three figures calculated in the top right chart - if an estimated for average room size was given.

In order to understand the effects of (apparently small) changes (e.g. extend daily core time by 30 minutes), the user has the possibility to create three experiments (each on a separate tab) which are immediately compared to the master data. Each of these experiments (see Fig. 4.20) can be saved separately, so that the user can come back later and continue experimentation.

The charts within the experiment-tabs are structured as within the master data tab - with two distinctions. First, every bar from the master tab is now replaced by a triple of bars, representing master data (blue bar), experiment

\footnotetext{
41 "Ecological operation time" is an extended core time defined as six days per week with 14 room-hours/day/room.
} 


\section{more space $^{\text {sact }}$}

Stammdaten | Experiment 1 | Experiment 2 | Experiment 3 |

Erfassung der Daten

Branche ?
$\odot$ Universität $\odot$ Schule

Betriebszeiten:

Verfügbare Räume * Raumgröße ?

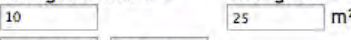

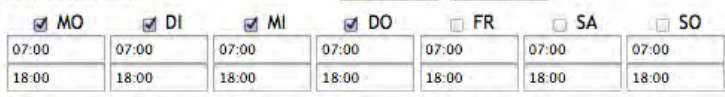

• Anzahl notwendiger RZF: a

- Anzahl Veranstaltungen: a

Veranstaltungsdauer: $a$

Externe Veranstaltungen

innerhalb der Nutzungszeit:

Externe Veranstaltungen

$\begin{array}{lll}\text { Externe Veranstaltungen } & \text { Stunden } \\ \text { außerhalb der Nutzungszeit: } & * & 0\end{array}$

Stammdaten speichern
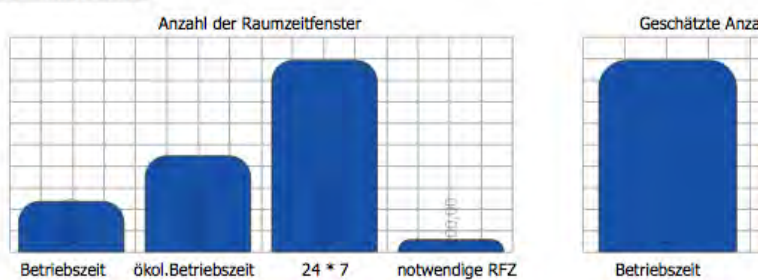

eschătzte Anzahl an minimal notwendigen Räumen

$100 \quad$ Stunden
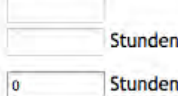

Auslastung der Räume in Prozent

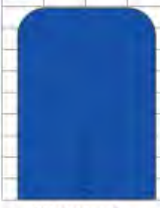

Betriebszeit

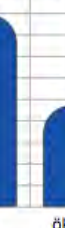

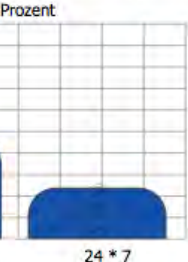

Figure 4.19: Master tab of static analysis tool (STAN, version "Basic"), intended to facilitate system understanding and awareness raising for impact of space utilization.

data (green) and the difference (red; absolute values). Second, the lower left chart has one additional value-triple which shows utilization benchmarks for core time, ecological operation time and 24/7-utilization ${ }^{42}$.

While STAN Basic is intended to facilitate system understanding and the impact of (increased) utilization efficiency, it is very imprecise. The features helpful in reducing the entry threshold are obstacles to more detailed and fact-based analysis, as all data is entered as accumulated values with little opportunity for fine-tuning. For example it does not differentiate rooms and events in terms of capacity, requirements or the like. STAN Advance was implemented with the goal to offer a benefit to users who understand the idea and possess the data necessary to evaluate space utilization.

To run the Advanced version the user needs two data sets: one describing its room structure and the second one describing the events within the system. In order to offer cross-platform access data is uploaded via web-GUI as csv-files. These files have to have a separate line for every entry (i.e. room or event) with the first line being the header and the second line the first data point. The columns of the two files are then

\footnotetext{
${ }^{42}$ These values are based on informal expert-knowledge and constant (i.e. independent of master or experiment data).
} 


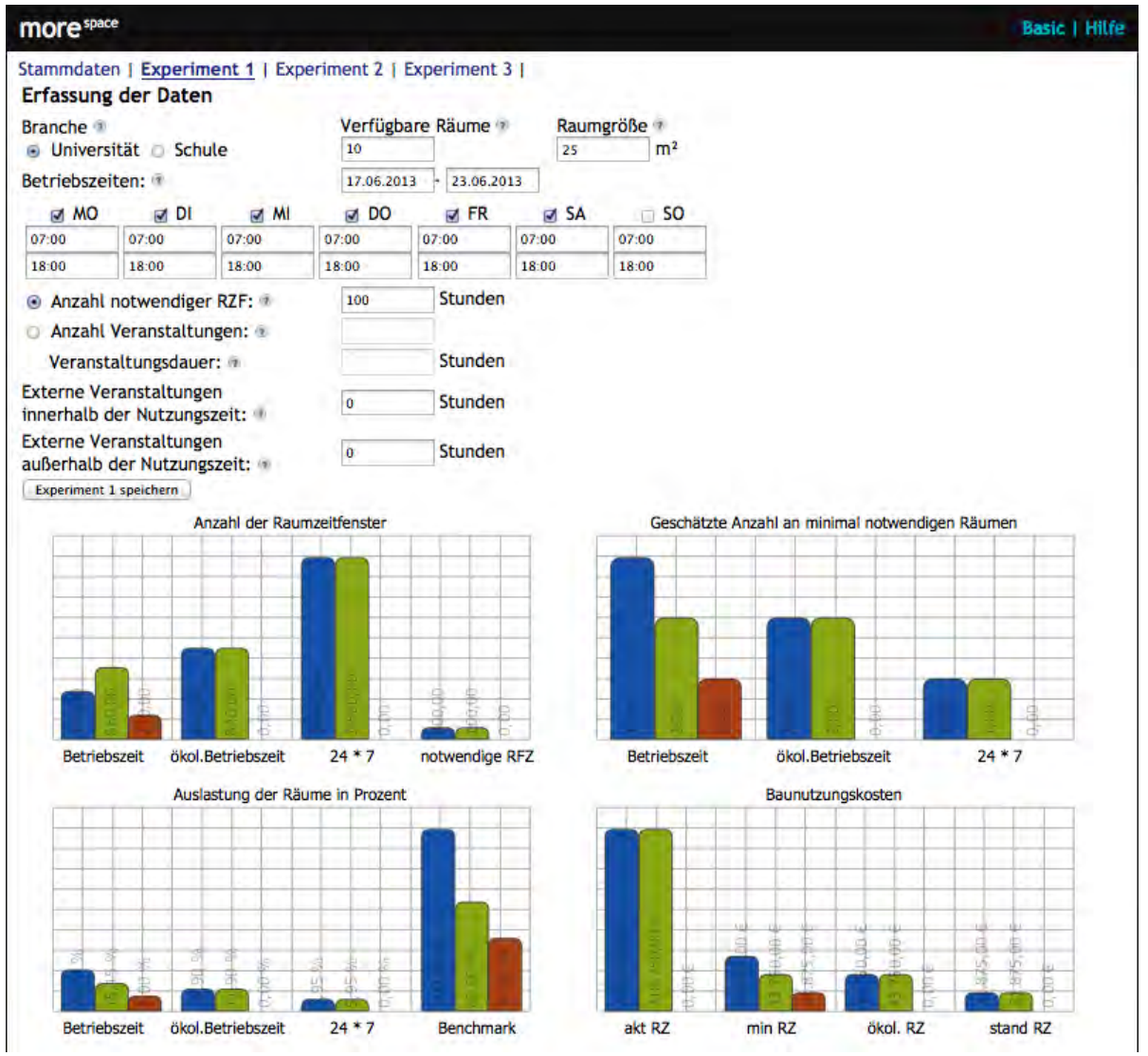

Figure 4.20: Experiment tab of STAN-GUI (version "Basic") designed to easily compare master data with up to three scenarios.

- room data

- ID

- capacity

- type of room

- size of room (square meters, optional)

- event data

- ID

- minimum required capacity

- required type of room

- start of event (dd.mm.yyyy hh:mm)

- end of event (dd.mm.yyyy hh:mm)

- type of event

This data is uploaded using the web-interface depicted in Fig. 4.21, processed and the results returned in a similar manner as familiar from STAN Basic (see 


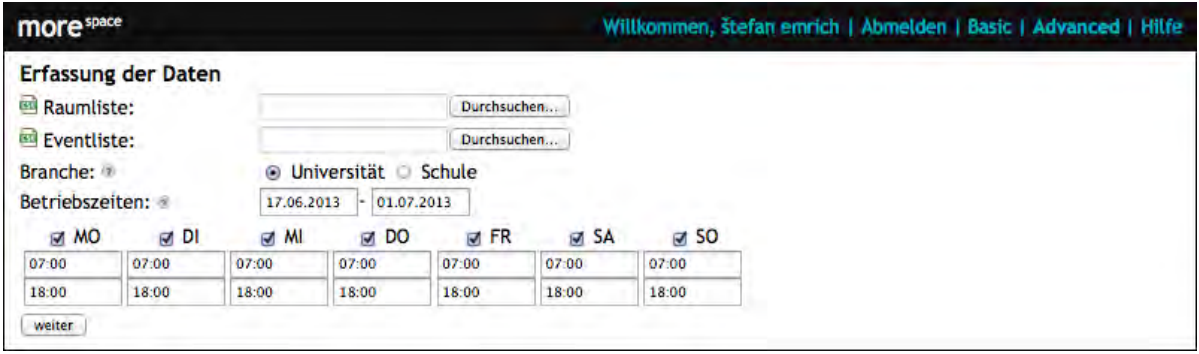

Figure 4.21: GUI of STAN "Advanced" which relies on extensive data transmitted via csv-files.

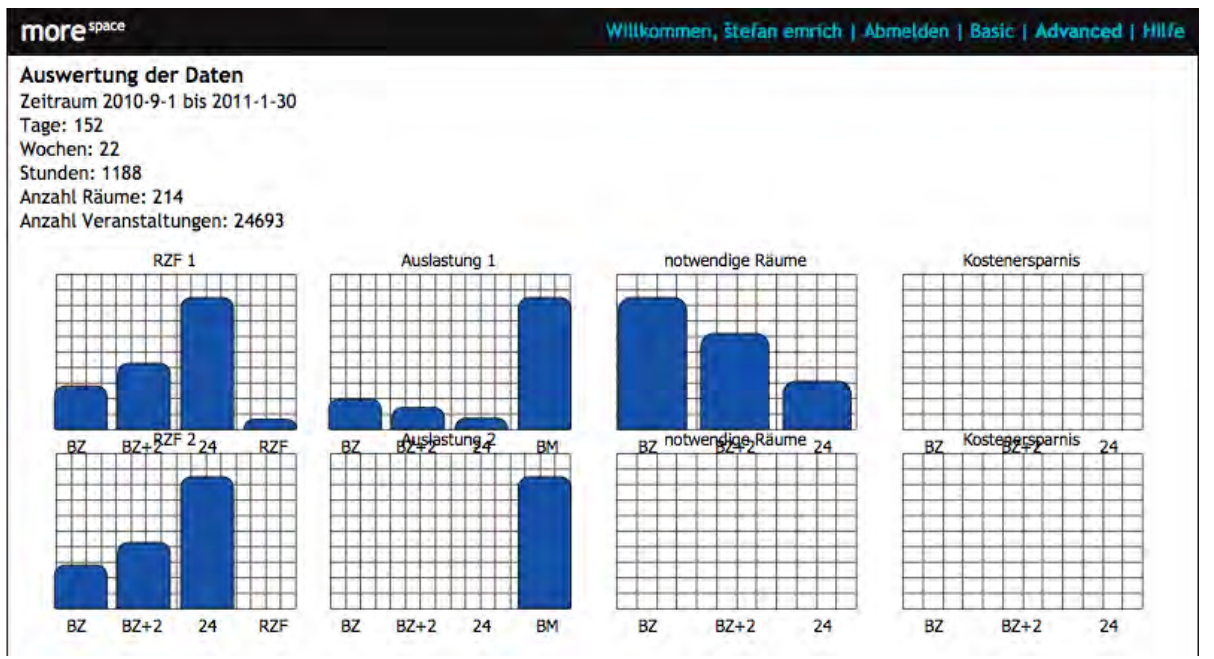

Figure 4.22: Results of STAN Advance for room-data with two categories.

Fig. 4.22). Although instead of in two rows all four charts are displayed in a single row, with a separate row of charts for each room type. In the data used to generate Fig. 4.22 two types of rooms have been used, while events only requested one type. Thus the second and third chart of row two remain empty (spare for the reference-benchmark). The right chart is empty for both rows as the room data did not contain (optional) room size. In this way the Advanced version of STAN allows to quickly analyze real world data of large systems for its improvement potential. 


\subsection{Simulation of the Office Environment}

The encountered rigid system (described in the previous sections) motivates this sections digression. As such rigidity is often found in bureaucratic systems (such as universities or governmental institutions) it leads into the field of office buildings' space utilization.

Based on the brief analysis of the field in section 4.3.1 a hybrid simulation model (consisting of DES and AB-methods) is developed in section 4.3.2. In the last part of the chapter (section 4.3.3) this model is being used to analyze of some general characteristics of such office systems.

The model presented in this last section of chapter "Application and Case Study" is to be understood in two ways. First, it should be seen as a complementary element to the deployed model for increase in space utilization within universities/educational systems. Second, it serves as an outlook on mathematical modeling in the area of space management in general.

\subsubsection{Space, Workplaces and Utilization}

Usually in private business the cost factor is even more of a topic than in governmental institutions. Nevertheless there is a certain blind spot when it comes to space related costs, which is partially induced by the status of owning representative buildings or a spacious office. But aside from this, the awareness for the potential savings (regardless whether of GHG emissions or financial ones) through an efficiency increase in space management is not yet existing. Subsequently current approaches to reduce space related costs focus on buildings' operating costs.

\section{Potential for improvement}

The effect of this focus is illustrated in Fig. 4.23 and has been described by Zitter et al. (2011). Operating costs account for only $20 \%$ of the annual buildingrelated costs ( $1^{\text {st }}$ bar in Fig. 4.23 ; left to right). They then provide benchmarks according to which roughly $40 \%$ (of the initial $20 \%$ ) are capable of being influenced - thus $8 \%$ of the total costs $\left(2^{\text {nd }}\right.$ bar). It is further possible to reduce the influenceable costs by $30 \%\left(=2.4 \%\right.$ of total costs; $3^{\text {rd }}$ bar $)$. Assuming a realistic reduction of $50 \%$ in practice, the total costs can be reduced by a mere 1.2 percent $\left(4^{t h}\right.$ bar)! It is thus obvious that this approach cannot contribute to substantial savings.

On the other hand buildings are used only for a fraction of their life time. As explained by Ottomann (1994) effective utilization of office space is only around $5 \%$. This includes a working week of five 8 hour work days, holidays and vacation of employees, breaks, sick leaves and social as well as organizational activities.

It is apparent that an efficiency increase in space utilization offers a far bigger potential for savings than reductions of operating costs. A theoretical increase of 5 percentage points (i.e. from $5 \%$ to a utilization of $10 \%$ ) does equal cutting the space required in half - and thus reducing space-related costs by approximately $50 \%$ ! This is illustrated by following example: A company with 100 offices has a utilization of $5 \%$. Availability of hundred offices per week (7 days á 24 hours) is 16.800 office-hours $(100 \times 7 \times 24)$. Utilization of $5 \%$ means that a mere 840 


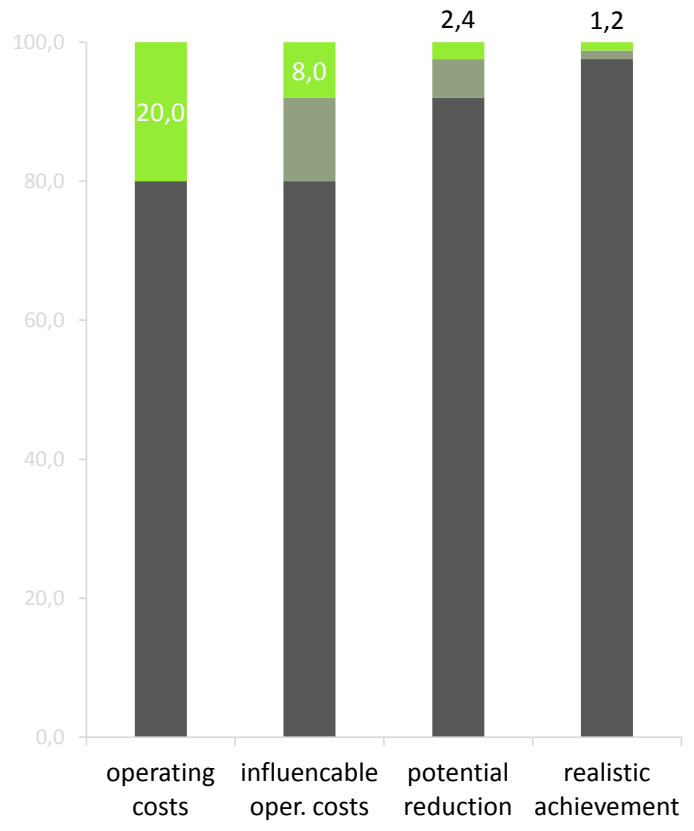

Figure 4.23: ATTENTION: change influencable to influencEable! Practically achievable cost reduction by tweaking of operational costs.

office-hours are actually "consumed" by the employees. As the actual need (840 office-hours) is not changed by a more efficient space-management, a raise to $10 \%$ utilization efficiency would require an availability of only 8.400 office-hours $(840=10 \% \Rightarrow 100 \%=8.400)$, which calls for $(8.400$ divided by 7 days á $24 \mathrm{~h})$ 50 offices - a $50 \%$ reduction of the original 100 offices.

The question that arises is: "How can (office) space be used in a more efficient manner?" - Which is equivalent to that of how much space is truly needed.

\section{Static Approaches}

To answer this questions decision makers need to know how much space is truly needed. This of course is hard to answer without adequate information. Approximations can be derived by rule of thumb estimates customary in the particular trade, although they will remain (rough) estimates. Chances are that the need for space will be over- or underestimated. Both outcomes come with significant costs (see Kovacs et al., 2013, for financial insight on inefficient utilization of office buildings). Either there will not be enough space for all employees, which not only requires renting additional space, but also disrupts workflows and thus decreases overall productivity. Overestimating required work space, on the other hand, leads to sub-optimal utilization. The situation improves less than it could have.

Trying to improve the results, more detailed calculations could 
be carried out. Nevertheless these will become extremely complicated and complex when trying to incorporate different behavior of employees. For example will sales representatives have needs different from in-house account managers, who will again have needs, working- and vacation times that differ from those of the IT-staff. Getting exact results under such heterogeneous conditions is challenging, to say the least. In addition, even if it would be possible to obtain exact results for this problem, they would be valid only for this one scenario. A change within the employee structure or a different space management strategy would require starting from scratch, as all calculations and consideration have to be applied to the new scenario. Another flaw of this approach is that it neglects the stochastic nature of the observed system (i.e. employees are not robots that have ever repeating, non-changing routines within their work-cycles).

Another approach is to closely monitor and track the employees' actual work place needs and use the data obtained for statistical analyses (e.g. electronic monitoring of workplace activities, collecting information on employee position, etc.). Nevertheless, this approach has some major drawbacks. First it raises issues regarding privacy. And even if legal it is likely to cause bad blood among employees and/or staff associations. Second if monitoring systems are not installed yet, it is costly to do so. Further it takes a long period of time to acquire sufficient amounts of data. Third data gathered is, by definition, always historic - even in real-time systems. Thus it can only be used to explain and analyze (management) strategies, employee structures and office layouts that have existed and been monitored in the real world (i.e. those from which the data comes from). But the data is only of very limited use when trying to understand the effects of alternative scenarios (e.g. modified employee structure, different working times, changed space management, etc.).

These above two methods (rule of thumb and statistic analysis) are regarded static models as they do depict the system behavior, but without any change over time. This is not to be confused with an (in)ability to "predict" the future state of the system. But the prediction does not change over time, as well as the models itself do not change their states (compare to section 2.1.2).

- Quoted from Emrich et al. (2013).

\subsubsection{Model Implementation}

To overcome the limitations of static approaches a dynamic model - based on discrete event simulation (DES) and agent-based methods - is being developed. The factors that need to be taken into account when modeling space utilization in an office environment are fairly similar to those of the described university environment (see section 4.1.2). As explained in Emrich et al. (2013), these are:

- employee structure i.e. which employee types are within the system and how many employees of each type 
- employee behavior

i.e.

which working preferences and what kind of behavior do the employees have (e.g. working times, fraction of field work, etc.)

- office environment

i.e.

how many offices/workplaces of what type are available

- The space management in place

i.e. which rules have to be considered when it comes to assignment of work places, which workplaces are available for whom (employees or employeetypes), etc.

these rules strongly depend on the objective of the simulation

\section{Modeling Approach}

In such a setup the individual employee can be regarded as the smallest unit. It is her behavior that defines the simulation result, and subsequently it is necessary to depict the employees in the most accurate way. For this reason "top-down" approaches (such as statistical methods) are only of limited success: they describe the system as a whole - without giving respect to the interactions of the system internal elements. Agent-based (AB) methods, as used in the present case for development of the "More-Space Office Tool", are producing the system's behavior via definition of its smallest units and their respective interactions - the employees and their behavior. AB modeling is treating every instance (i.e. employee) as an independent entity with an individual behavior (see section 3.3.3).

Further, to recreate realistic behavior, the stochastic nature of events has to be incorporated into the simulation model. This is necessary as, for example, employees will not come to work every day at exactly 8:00 A.M. On the contrary they will most often come a bit earlier or later as they have to deal with "unexpected" events, such as traffic jams or delayed public transport. Such events can potentially trigger chain reactions (e.g. missing the first of a series of connections by only a second can lead to a cumulative delay of several hours) and are thus vital for the dynamic nature of the model. Discrete event simulation (DES) is aiming at such problems and is therefore incorporated into the model.

- Quoted from Emrich et al. (2013); reformulated.

In order to combine the features of AB methods and DES a hybrid model was created using the simulation environment AnyLogic, which is based on the object oriented programming language JAVA and capable of supporting both approaches (AB and DES).

\section{Employee}

Within the model each employee is modeled as individual agent in a class called "Worker". This agent has several parameters and variables and a combined 
statechart for its health- and work-status (see Fig. 4.24). The parameters of the object Worker are:

- employeeType name of employee type (e.g. customer support or developer)

- employeeColor the color of the agent's visual representation

- daysInOffice the number of days/week which the agent is working in the office, (e.g. customer support might come into office only once a week and be at customers' locations the remaining days of the week)

- timeInOffice the average duration the agent stays in the office, once it comes to the office

- startShiftMin the earliest time that the agent will come to work (if it comes to work), i.e. earliest time to start its shift

- startShiftMax the latest time that the agent will come to work (if it comes to work), i.e. latest time to start its shift

- fixedWP

boolean parameter if the agent owns a fixed (exclusive) workplace or not

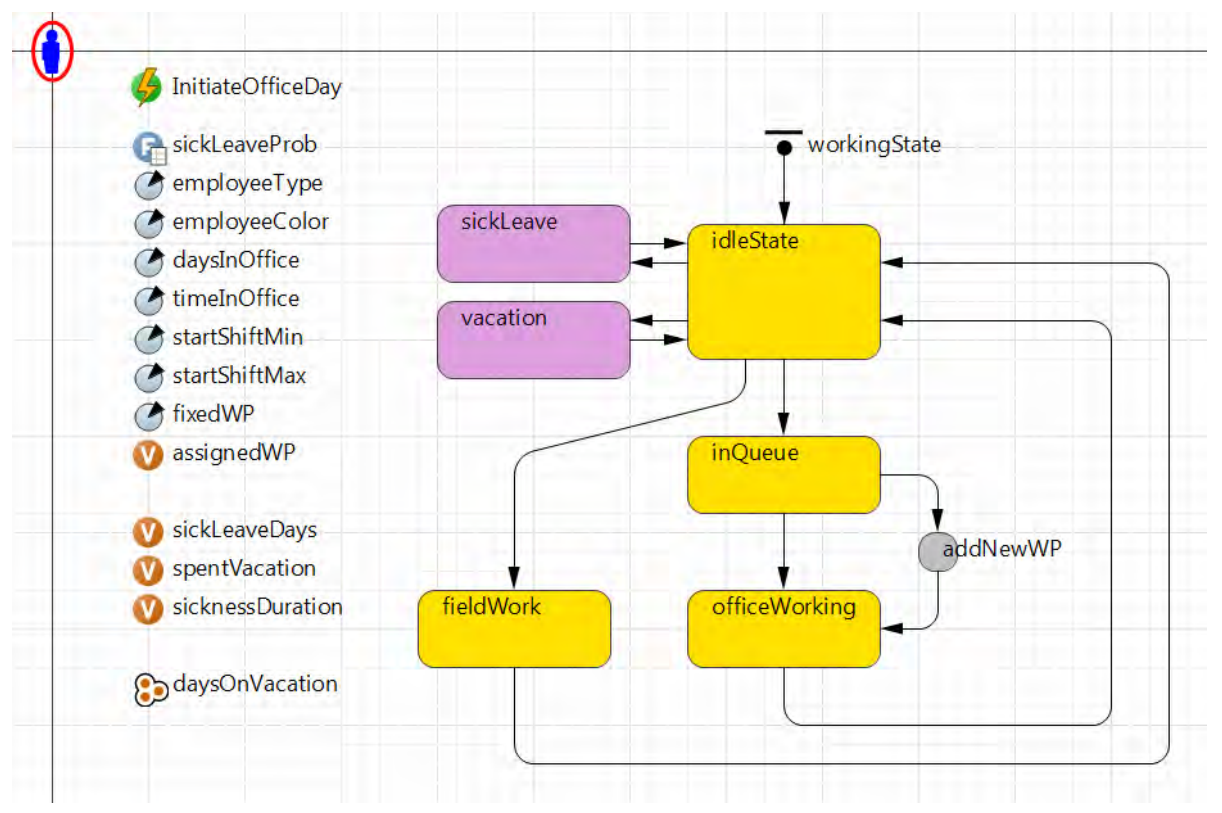

Figure 4.24: Structure of Worker object in the model, including parameters, variables, statechart and control-elements.

And its variables are: 
- assignedWP

$\mathrm{ID}^{43}$ of the workplace currently assigned; if fixedWP is true this ID is constant throughout simulation

- sickLeaveDays

counter of days the agent was on sick leave

- spentVacation

counter of vacation-days consumed

- sicknessDuration

used to store the duration of the sickness if the agent turns sick

Besides the statechart and the above variables and parameters the Workeragent is composed of three more control elements that are used to control the agent's behavior. These are daysOnVacation (list), sickLeaveProb (table function) and InitiateOfficeDay (dynamic event) and will be looked at in more detail later.

As mentioned the statechart used combines the working state and the health state of the agent. The reason for the combination is the assumption of their mutually exclusive nature. I.e. that an employee turning sick is not going to go to work. For simplification of the model it is further assumed that an employee is not turning sick during vacation or while at work. The top arrow indicates that the agent enters the statechart into state "idle" (idleState). This is the initial state from which every work day is started. From here the agent starts its working day either as an office day (via inQueue and officeWorking) or working outside the office (fieldWork) before it returns to the idle state. In case that the agent turns sick or takes a day off it changes from idle into state sickLeave and vacation respectively. The last remaining state, addNewWP, will be described later.

At initialization of the simulation each agent's daysOnVacation-list is filled with 25 days $^{44}$ on which the agent is on vacation. These 25 dates are scheduled randomly with following constraints:

- For $50 \%$ of all employees a blocked vacation is being scheduled, ...

- with a length that is uniformly distributed between 10 and 15 days, and

- which starts randomly (uniformly distributed) within a user-specified "core vacation period" (e.g. summer holidays).

- For $40 \%$ of the remaining employees (20\% of total) a blocked vacation (of uniformly distributed length between 10 and 15 days) is scheduled at a random time (unif. distrib.) during the year.

- Finally each employees vacation list is filled up (until 25 days are reached) with random (unif. distrib.) days.

\footnotetext{
${ }^{43}$ Remark: pointer to object instance.

${ }^{44}$ By Austrian law employees are entitled to an annual vacation of five weeks. Hence people working five days per week (the majority) receive 25 days of vacation (per year). People working 6 days per week subsequently receive 30 days.
} 


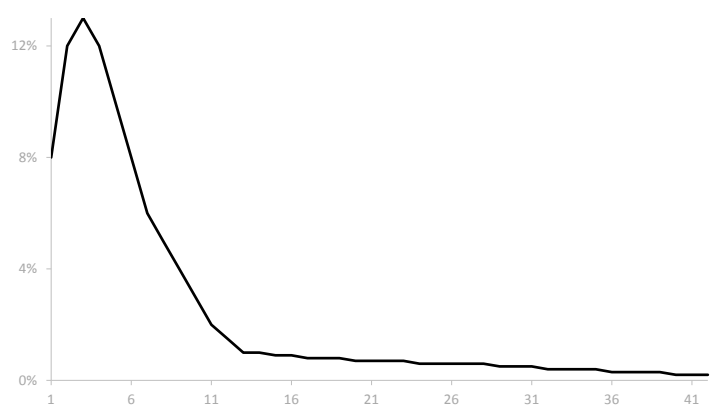

Figure 4.25: Skewed bell-shaped distribution of sick-leave duration used within the office model.

To create a closer to reality behavior of employees a sickness function has been developed. Based upon several data sets from "Statistik Austria" (the Austrian Statistical Central Office) a distribution of the duration of sick leaves has been developed (see Fig. 4.25). This distribution is naturally only a rough estimate, but sickness duration and frequency strongly depends on the business field and the region/country and can thus not be modeled precise and generally valid at the same time. Instead it is recommended to use real-world data for parametrization and model fitting.

\section{Employee Structure}

With the basic employee being defined in the above described, flexible way, one object class (Worker) can be used to represent different employee types. At initialization of a simulation run the user is presented with a GUI (see Fig. 4.26) that allows to define the numbers of employees per employee type, the core vacation period as well as choosing between flexible and fixed (i.e. individual, not shared) workplaces.

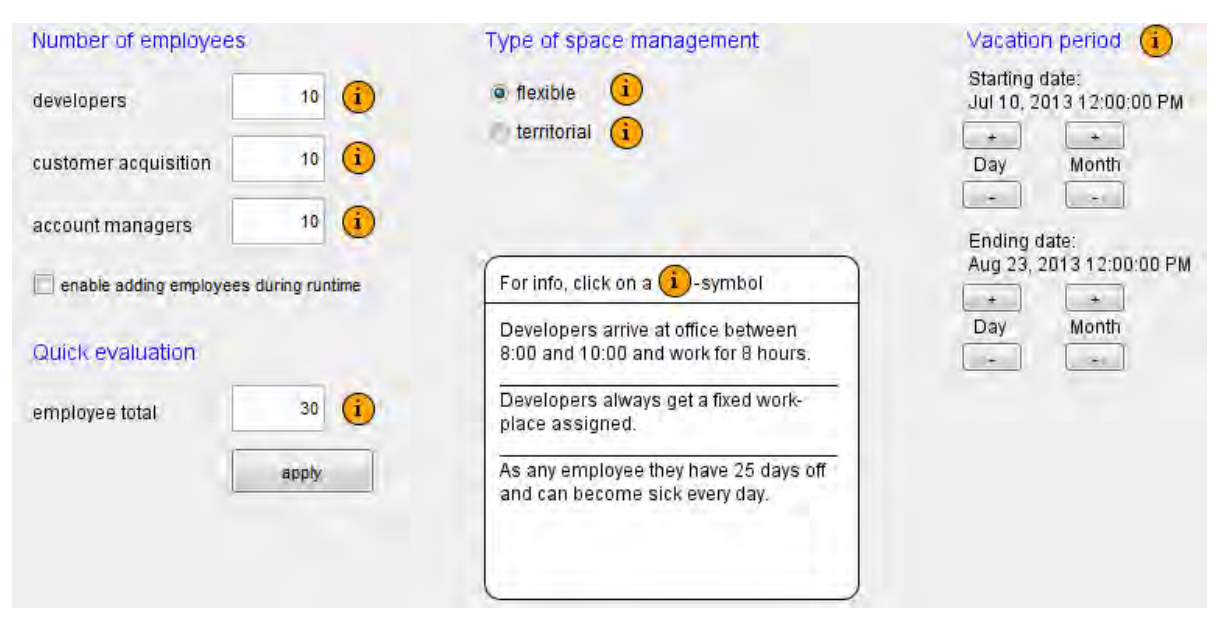

Figure 4.26: Section of GUI that allows specification of main simulation-parameters.

The model then generates the specified number of employees per employee 
type (in this case three types are hard-coded) together with their specific characteristics (see parameters, listed before).

An alternative possibility is to control the employee structure using spreadsheet-files, which are loaded by the model at initialization. This approach maximizes flexibility and empowers users without programming skills or access to the source code to precisely control the employee structure. In this case the model processes the spreadsheet-file row by row and creates an employee type (with multiple instances) for each of these. The exemplary spreadsheet depicted in Fig. 4.27 leads to the creation of three different employee types: "Developers", "Acquisition" and "Forenoon", with respective behavior.

\begin{tabular}{|c|c|c|c|c|c|c|c|c|c|}
\hline 1 & A & B & C & D & $\mathrm{E}$ & $\mathrm{F}$ & G & H & 1 \\
\hline 1 & \multicolumn{6}{|c|}{ Employee statistics } & & & \\
\hline \multicolumn{10}{|l|}{2} \\
\hline 3 & & & & arrival & office & & & agent col & \\
\hline 4 & type & No. of employees & days in office & earliest & latest & $\begin{array}{l}\text { time in } \\
\text { office }\end{array}$ & red & green & blue \\
\hline 5 & & & days/week & hour & hour & hours & $0 . .255$ & $0 \ldots 255$ & $0 \ldots .255$ \\
\hline 6 & Developers & 30 & 5 & 8,00 & 10,00 & 7,00 & 255 & 165 & 0 \\
\hline 7 & Acquisition & 30 & 1 & 8,00 & 10,00 & 2,00 & 245 & 20 & 147 \\
\hline 8 & Forenoon & 40 & 5 & 7,00 & 17,00 & 2,00 & 84 & 84 & 84 \\
\hline 9 & & & & & & & & & \\
\hline
\end{tabular}

Figure 4.27: Spreadsheet file controlling model-internal employee structure.

With this approach it is even possible to create an individual class for every single employee and thus incorporate individual behavior (e.g. by adding preferred vacation times and individual, age- or gender-dependent illness probabilities). Besides potential privacy concerns this naturally requires to have the corresponding data in the first place. In relation to the benefit for the simulation result this approach is most likely too costly and thus not reasonable to pursue.

\section{System behavior}

In the current implementation the general goal is to use the model to calculate the number of required workplaces - for a given employee structure - and in this particular case to evaluate the savings potential compared to fixed workplaces ${ }^{45}$. With the employee structure and behavior in place, the next step is to model the general system behavior.

At initiation the model creates all employees as instances of class Worker, which, when coming into office require a workplace. Subsequently the number of required workplaces can be obtained by adding a workplace to the (virtual) building each time one is needed. This approach is supported by the object oriented architecture of the simulation environment. It is possible to create a new instance of a class at runtime. Thus offices and workplaces are implemented as classes (Office1WP office with one workplace, Office $2 W P$ with two workplaces and Workplace). When a Worker changes its state from idle to inQueue a check for free workplaces is performed. If a workplace is available the employee uses it, if not a routine is called which creates an additional instance of Workplace

\footnotetext{
${ }^{45}$ I.e. a workplace model in which every employee has an individual workplace that is not shared
} 
and assigns it to the employee. Remark: Since employees with fixedWP $=$ true do not release their workplace (ID), it also cannot be taken by other employees.

The structure of class Workplace is a fairly simple one. Besides information relevant for visualization purposes (e.g. its coordinates) it has following three variables:

- assignedEmployee analogous to Worker's assignedWP this holds the ID of the employee that is assigned to the workplace; in case of a fixed workplace the $\mathrm{ID}^{46}$ does not change

- nTimesUsed counter for utilization analysis which registers ever use-session

- $\mathrm{nMinUsed}$ counter for utilization analysis which registers every minute of usage

As indicated the model has two more object classes: Office1WP and Office2WP. They represent the frame in which workplaces are set. Depending on the 1WP or $2 \mathrm{WP}$ the object houses one or two workplaces. The primary (and sole) reason for their existence is the visualization of the simulation.

During simulation a cyclic event wakeAgents, scheduled for 00:01 of each simulated day, triggers the actions of all Worker-instances. It schedules their dynamic events InitiateOfficeDay, depending on probabilities and other factors. First it checks whether the current day is a workday or not. If it is, it checks how many days per week the respective agent is working "in office" and probabilistically determines whether a day in office or a day of field work is scheduled.

The exact instance when the (employee-internal) dynamic event InitiateOfficeDay is taking place is scheduled with a uniform distribution between the agent's startShiftMin and startShiftMax. When this point of time is reached, this internal dynamic event performs a state-check on the agent (if healthy and not on vacation) and then (probabilistically) determines whether the agent becomes ill or not - in which case it proceeds to work in office.

\subsubsection{Simulation and Findings}

\section{Parametrization}

Without real-world data to derive an employee structure from and with no benefit of a super-realistic one, a simplified employee structure was used to evaluate the savings potential of a flexible space management compared to a fixed workplace model. Nevertheless expert-knowledge was used to obtain a close-to-real employee structure and behavior.

The structure consists of developers, employees in customer acquisition and account managers, of which only the first are granted a fixed workplace. This is explained by their respective "in office" working times. Developers come to office (if not ill or on vacation) on all workdays, arrive in office between 8:00 and 10:00 (a.m.) and work for 8 hours. Customer acquisition personnel spend four days per week with field work and hence only have one day in office. When they are in office they, as developers, come between 8:00 and 10:00 and work for

\footnotetext{
${ }^{46}$ Remark: pointer to object instance.
} 


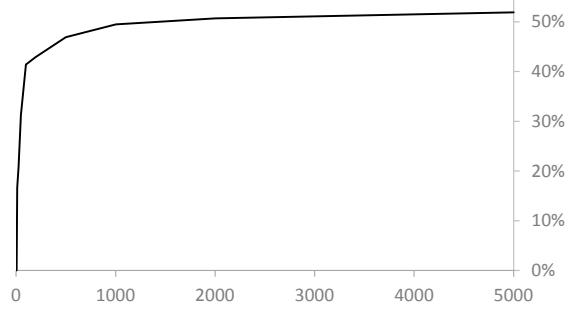

(a) Linear x-axis

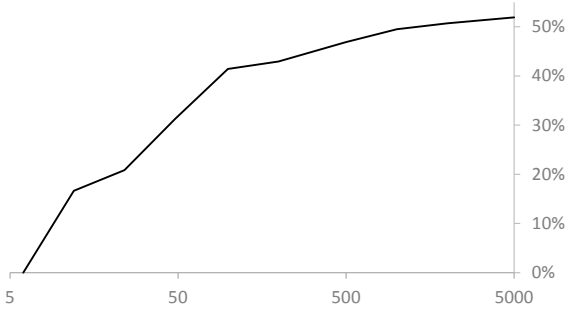

(b) Logarithmically scaled x-axis.

Figure 4.28: Workplace savings potential (y-axis) as a function of company size (xaxis) - plotted on a linear (left) and a logarithmic scale (right).

8 hours. Account managers are doing mainly field work, but come into office daily, although at irregular and changing times. They arrive between 8:00 and 17:00 and are then in office for two hours.

\section{Results}

For evaluation of the savings effect through flexible workplace utilization a balanced structure with $1 / 3$ of every employee type was used. Flexible workplace utilization was defined in such a way that all employees (except developers) use any free workplace (except those that are assigned to a developer); developers always use their assigned workplace.

The model was used to simulate the workplace requirements of each company size over one year (365 days). To compensate the effect of outliers the Monte Carlo method was applied. For every company size 10 simulation runs (each with a random seed for random number generation) were produced and averaged. The savings potential is then calculated as the difference between the simulation average and the company size ${ }^{47}$. The results are shown in Fig. 4.28, once on a linear (Fig. 4.28b) and once on a logarithmic scale (Fig. 4.28a). It is obvious that small enterprises can draw no and medium-sized ones only limited benefit of a flexible use of workplaces. Large companies on the other hand can cut more than $50 \%$ of their workplaces, compared to a fixed model!

Arguably averaging of simulation results leads to a lower number of workplaces than required in the "worst case" scenario. But then again flexible use of workplaces always holds a theoretic danger of shortage: in the absolute "worst case" all employees require a workplace at the same time. The question that has to be answered in practice is: how much risk does one want to take? Depending on the answer it is necessary to plan with a sufficiently large buffer. In addition the difference between mean and maximum is very small (see tab. 4.4), which is explained by the (fairly long) runtime of the basic simulation (365 days). The odd employee numbers are explained by the employee structure, which consists of three equally large groups.

Using the variable nMinUsed of the Workplace-object it is possible to calculate the effective occupancy - either for every workplace, or for the whole lot. In doing so one must consider that the employees are modeled in such a way that

\footnotetext{
${ }^{47}$ As the number of employees equals the number of workplaces required if every employee has their individual workplace.
} 
Table 4.4: Simulation results for required workplaces: average and maximum (of 10 simulation runs) and difference.

\begin{tabular}{cccc} 
Company size & average & maximum & difference \\
\hline 6 & 6 & 6 & 0 \\
12 & 10 & 10 & 0 \\
24 & 18 & 19 & 1 \\
28 & 32 & 33 & 1 \\
99 & 58 & 58 & 0 \\
198 & 110 & 113 & 3 \\
501 & 260 & 266 & 6 \\
1002 & 501 & 506 & 5 \\
2004 & 981 & 988 & 7 \\
5001 & 2395 & 2405 & 10 \\
\hline
\end{tabular}

they do not leave their workplace until they finish their workday. I.e. there are no meetings, conferences, lunch-breaks, and the like - which would naturally reduce effective occupancy. Incorporation of such elements would require to consider whether an unoccupied workplace left for such a reason would become available (for use by another employee) or remain reserved although unoccupied by the initial employee. The present implementation has been chosen in order to avoid this problem.

Occupancy has been calculated in two different ways. Once the total time of workplace-usage is divided by the total simulation time (i.e. 24 hours, 7 days a week), the second time it is seen as fraction of the core time (10 hours per business day, i.e. Monday through Friday). With respect to the findings in Fig. 4.28 a company with 500 employees can already profit significantly of a flexible workplace management. Thus occupancy has been analyzed for this category by simulating a period of one year for 5 times with the flexible workplace utilization as previously described. I.e. developers, who are working full time, have a fixed place, the remaining employees not.

The simulation results (depicted in Table 4.5, labeled "fixed") show that the total occupancy (labeled "total") lies at about $19.7 \%$ and during core time (labeled "core") around $66.3 \%$. In a second step the flexibility of the space management has been increased by one notch: developers also use flexible workplaces (results labeled "flexible" in Table 4.5). Even though developers are working full time (i.e. 8 hours per day, 5 days per week) the impact that this change has is dramatic. Only by unblocking the (previously fixed) workplaces blocked during vacation and illness of developers total utilization was increased by one percentage point (relative: 5\%) and core utilization by 3.3 percentage points (relative: 5\%). Again (compare to tab. 4.4) the deviation of the results is so small that the ranges of the two settings' results never overlap. The explanation for this is again found in the long simulation period of 365 days, which causes graduation of a lot of random influences. 
Table 4.5: Comparison of workplace occupancy for scenarios "fixed" and "flexible"; simulation results for "total" occupancy and "core" time occupancy (10 hours/business day).

\begin{tabular}{cccccc} 
& \multicolumn{2}{c}{ total $(\%)$} & & \multicolumn{2}{c}{ core $(\%)$} \\
\cline { 2 - 3 } \cline { 5 - 6 } sim-run & fixed & flexible & & fixed & flexible \\
\hline 1 & 19.88 & 20.87 & & 66.87 & 70.19 \\
2 & 19.51 & 20.11 & & 65.61 & 67.66 \\
3 & 19.63 & 20.87 & & 66.02 & 70.21 \\
4 & 20.03 & 21.14 & & 67.38 & 71.10 \\
5 & 19.29 & 20.48 & & 64.89 & 68.87 \\
\hline average & 19.70 & 20.69 & & 66.28 & 69.61 \\
\hline
\end{tabular}

\section{Conclusion}

Calculation of required number of workplaces is a queuing theory problem such as establishing the number of checkout counters in a post office or supermarket and general dimensioning problems in the area of service provision. With the above described hybrid approach (combining AB methods and DES) it is possible to model workplace utilization at an employee-based level, which has several advantages.

One major advantage is the transparency of the model and hence of the simulation results. It allows the user for whom simulation is carried out to understand and follow the reasoning behind the model, without becoming a simulation-expert herself (e.g. statecharts are easily read and understood). A second is the closeness of the attribute-mapping between implementation and reality, which at the same time is partially responsible for the before mentioned. An employee turning ill is implemented as agent-internal state change from "healthy" to "ill".

Besides the easy interpretation of the implemented model, the object oriented approach also allows for a very flexible and easy modification and adaption. If additional requirements arise they can more often than not be incorporated in a very efficient way (e.g. fine-tuning of agents' behavior, adding of attributes to objects, introduction of additional statistics, etc.).

With the model being of academic nature, the focus of it lies on serving as a proof of concept. These results obtained are thus not carved in stone, as they are a product of employee structure and behavior as well as of the space management in place and of simulation parametrization. All of which was based not on real data but on assumptions, which, although chosen with a claim for authenticity, reflect the simplifications accepted to obtain a slender model.

Against the background of current practice in space management of office buildings (see sec. 4.3.1) the model results point at a huge potential for improvement. Amongst others this requires raising of awareness for the issue, which can be supported by such conceptual models. On the other hand the model already incorporates most of the aspects necessary to conduct analysis of real systems and only requires for appropriate parametrization. In case of potential 
extensions, the flexible (object oriented) architecture allows for very efficient adaption. Finally, the big question and challenge that remains is whether an institution is willing and capable of incorporating the required changes of business processes, i.e. install a flexible space management. Without this step the potential for improvement is more than limited. 


\section{Chapter 5}

\section{Conclusion \& Perspective}

\section{Summary}

Amidst global warming and financial crisis the reasons to cut back the waste of (natural as well as financial) resources are evident. Nevertheless, simply "cutting back" is too short sighted to solve the problem. Solving the problems faced requires to change the perspective from black \& white to the shades of grey in between: putting the "footprint" in relation to the benefit generated by its input, as explained in chapter 1.

This perspective has the power to change the way in which "eco-friendly" is being discussed in real estate management. If the footprint of a building is not calculated as an end in itself but distributed upon the "units of benefit" generated within this building, then it is more than obvious that the buildingspecific "costs" for the production of one unit can be reduced in two ways: (1) decreasing the footprint or (2) generating more (units of) benefit.

While option (1) is currently being pushed by governments and society (e.g. funding for thermal insulation, prestige advertising of "green buildings"), it is illusory belief that it will be sufficient - and sufficiently fast - to cope with the problems faced. Macroeconomic reasoning (keywords: rate of new construction, ecological problems of insulation materials), as elaborated in chapter 1, strongly favors option (2).

As units of benefit can also represent entities that are not (mechanically) produced, but of an abstract nature (e.g. people sheltered in a residential building, productive hours of staff in an office building, or students taught at a university), generating more benefit becomes directly translated to an increase in space utilization. Subsequently an increase of the generated benefit is equal to an increase of space management efficiency. Nevertheless, currently utilization of built space is still a more-or-less unidentified topic. Despite the fact that things are slowly starting to change, extremely low utilization rates prevail (HEFCE, 1999; Wiegand et al., 2007a) and utilization of mathematical simulation models to improve space management is practically non-existent.

In order to successfully deploy mathematical simulation models within this field, a potential building has to fulfill several criteria. For example does it not make sense to try improving (very) small buildings, or ones with a rigid and extremely homogeneous utilization (e.g. an office where all employees enter at 8 in the morning and leave at 17) because of their extremely limited margin. For 
this reason the thesis' focus was initially set out - primarily but not exclusively - on university- and university-like buildings, as these

- are sufficiently large,

- exhibit diverse, heterogeneous utilization,

- and offer great savings potential (HEFCE, 1999, see).

It is worthwhile adding, that - most likely due to these characteristics - university space management is one of the areas that receives the most attention from mathematical perspective. Technically the room-to-course or room-toclass $^{1}$ scheduling problem of universities can be interpreted as assignment of a "work piece" to a "server", and vice versa, with certain constraints that have to be respected. As described in section 2.2.3, such scheduling and timetabling ${ }^{2}$ problems can be approached either via exact algorithms or by heuristics. Regardless of the approach, only constraints that can be formalized may be taken into account by the algorithms. And, with the general course timetabling problem being NP-hard (see MirHassani and Habibi, 2011), each constraint adds to the complexity of the problem.

While methods for such timetabling-, scheduling- or resource-allocation problems exist, they are found in practice mainly in the industry (especially in production and logistics) or for "plain" timetabling (e.g. organization of night shifts). At universities their application is scarce, if existing at all (Carter and Laporte, 1998). One of the main reasons for this is the necessity for incorporation of so-called "soft constraints" (e.g. personal preferences or individual requirements - see Piechowiak et al. (2005)). These are especially problematic as sometimes even formalization is not possible, and because they add a human dimension to the problem, which is best described by Carter (2001) as "practical course timetabling is $10 \%[\ldots]$ theory, and $90 \%$ politics! ... 'You cannot dictate to professors when they will teach courses!'”.

More criticism of the current development of timetabling comes from Piechowiak et al. (2005) who state that "fully automated tools are not efficient when the constraints cannot lead to a valid solution. In these situations, the tools do not provide any support in explaining the causes for the lack of solution. Nothing is given to determine which constraints must be relaxed to bring about a solution. The quality of these timetables also depends on the exhaustiveness of the constraints. In a university, it is impossible to collect and to formalize all this information. Expertise of timetablers is the key." And McCollum (2007) even goes as far as to argue that "researchers, in many cases, have been working on oversimplified problems".

Concluding from extensive literature review, one can state that the problem of university course timetabling is still not solved satisfactory (at large scale) and identify following main reasons:

(a) Approaches or problem-sets are too specific or simplified.

(b) The human factor is being neglected - as a constraint or in politics.

(c) Deployment of models is not taking place - for unknown reasons.

\footnotetext{
${ }^{1}$ And the other way round: course-to-room and class-to-room, respectively.

${ }^{2}$ In the following they will be referred to as timetabling only.
} 
It is the author's assumption that the (interdisciplinary) complexity of integrating models/software in real-world systems is one of the major reasons for reason (c). This belief is lead by analogous problems in software development $\&$ integration.

Thus space management (at universities) is not only a mathematically complex problem. Adding a second perspective to the formal problem (i.e. timetabling), seemed promising in order to come up with solutions for it. For this it was necessary to differentiate between

- space planning and space management and

- timetabling and room allocation,

where space planning denotes the long-term process (strategic planning on how much space has to be allocated for what purpose) and space management the short-term process of room allocation to cover existing demand. This, and necessary incorporation of the human factor, suggested shifting the focus from precise timetabling to the fuzzy problem of room allocation. An additional direction for investigation was pointed out by $\operatorname{HEFCE}(1999$, p. 43) who found that "for efficient space management [ ... ], the universities will require full information about their existing space, its capacity, its use, its level of space utilisation and costs which are attached to that space." I.e. they hinted that efficiency improvements are hindered by incomplete information and a lack of overview (because of incomplete or unstructured data) respectively.

Subsequently the thesis' main focus was set on two aspects. The first one being necessary (1) formalization of the frame for deployment of mathematical simulation models in the field of space management and planning (e.g. sections 4.1.1 and 4.2.1). I.e. setting up key definitions, units of measurements, mapping of modeling concepts onto problem (e.g. adequate interpretation for discrete event simulation (DES) of the room-to-course mapping), adaption of modeling methods (agent-based (AB) extension of DES to incorporate individual decisions) and the like.

The second aspect was to (2) address the problems of current approaches as described in section 2.2. As sketched above, the problem of (a) overly specific methods was softened by focusing on room allocation, which allowed for creation of a more flexible, generally usable tool (described in section 4.2). This also complied with the Austrian university system where timetables are coordinated by departments and hereby a product of many influencing factors, such as historical development and know-how, reaction to newly introduced branches of study, personal preferences of the faculty, etc. As such, the approach also takes into account (b) incorporation of the human factor. This is also promising as, according to Carter (2001), "giving timetable [representatives] the facility to make ... changes was the single most important contribution [to the goodness of solution]".

The problem of (c) model deployment was addressed with a multitude of approaches. Most importantly the general simulation process was expanded (sec. 2.1.3), which allows for a holistic problem approach. This made it possible to use and adapt methods from other research fields for thorough system analysis (section 3.1). In section 3.2 the concept of a deployment matrix has been developed (resting on knowledge from system analysis) in order to assist the deployment processes or to guide through alternatives if deployment is not 
possible as intended. Further information visualization methods were harnessed to extract knowledge from the large amount of data generated. And, as a byproduct, a definition for dynamic modeling has been developed and proposed (section 2.1.2).

\section{Results}

These methods have then been put to the test in a case study at Vienna University of Technology. Within a research project a hybrid model has been developed for increase of room utilization. The project once again proved that practical timetabling is in large parts politics. While the modified Business Process Modeling Notation (BPMN) allowed to define room-booking processes at the university for the first time, their institutionalization proved impossible (section 4.1.3). Nevertheless the developed deployment matrix allowed it to react and modify the project in order to make the best of a bad job. Based on static data analysis and information visualization, alternative decision support tools have been developed (section 4.2.2).

Utilization of these tools (including data analysis) made it possible to save an estimated $€ 250,000^{3}$. For the time of reconstruction of the Audi Max, the universities largest lecture hall, it was assumed that renting of backup capacities was necessary. But it was possible to prove that the university had enough resources to accommodate all lectures within the existing buildings - although with some necessary reorganization.

In addition the lessons learned during the project (i.e. the experienced rigidity of the observed system) triggered the development of a conceptual model for simulation of office environments (section 4.3). Although still at a conceptual level, the model was used to show the potential benefit (savings and efficiency increase) of flexible workplace management. In addition it offers a great possibility to serve as a tool for awareness raising of the problem and as a confidence building measure to use mathematical simulation for decision makers within the realm of space management.

\section{Outlook}

In the future society will most certainly be forced to hugely increase the efficiency with which it uses its resources. The building sector does not only rely on natural resources in the course of production - moreover it shapes the (ecological) footprint that its "products" leave for decades to come. Thus space management can help to not only increase efficiency of buildings "output" but even avoid the need for additional construction.

As mathematical modeling and simulation (MMS) is still underrepresented within this field, this thesis provides concepts necessary for application of MMS within it. In doing so it contributes to the development of a basis on which MMS can be deployed in the realm of space management - including, but not limited to, setting up a framework of definitions and units of measurements, as well as creation, adaption and further development of tools for model deployment (including system analysis and post-processing).

\footnotetext{
${ }^{3}$ Estimate by Mr. Amtsdir. Reg. Rat Gerald Hodecek, head of the university's department for building, construction and technology (GuT)
} 
Against the backdrop of a very low awareness for the problem of efficient room utilization, it is to be hoped that this will change in the future. As pointed out above, the developed tools can serve either as support for decision makers, as proof of concept for skeptics or as awareness raiser for a general audience.

With respect to the Austrian university system two recommendations can be issued. One is to implement a central, demand-based event-to-room allocation process (e.g. as proposed in section 4.1.2). The second one, aimed at the responsible politicians and governmental institutions, is to dissolve the conflicting interests of the "Bundesimmobiliengesellschaft", the Austrian universities and the Ministry of Finance (described in section 1.2.4, p. $17 \mathrm{ff}$.), which not only wastes money on overheads but also hinders efficient utilization of space. 


\section{Bibliography}

Aristotle. Metaphysics. The Internet Classics Archive, 350 B.C. (est.).

J. Banks. Handbook of simulation: principles, methodology, advances, applications, and practice. A Wiley-Interscience publication. Wiley, 1998. ISBN 9780471134039. URL http://books.google.at/books?id= dMZ1Zj3TBgAC.

Ethan Bernstein and Umesh Vazirani. Quantum complexity theory, 1997.

C. Beyrouthy, E. K. Burke, D. Landa-Silva, B. McCollum, P. McMullan, and A. J. Parkes. Towards improving the utilization of university teaching space. Operational Research Society, 60:130-143, 2009.

Camille Beyrouthy, Edmund Kieran Burke, J. D. Landa Silva, Barry McCollum, Paul McMullan, and Andre J. Parkes. Understanding the role of ufos within space exploitation. In Edmund Kieran Burke and Hana Rudová, editors, Practice and Theory of Automated Timetabling VI, volume 3867 of Lecture Notes in Computer Science, pages 359-362. Springer Berlin, Heidelberg, Berlin Heidelberg, 2007.

Camille Beyrouthy, Edmund Kieran Burke, Barry McCollum, Paul McMullan, and Andre J. Parkes. University space planning and space-type profiles. Scheduling on Applications and Methodologies for Planning and Scheduling, 13(4):363-374, 2010.

BIG. Bundesimmobiliengesellchaft, June 2013. URL http://www.big.at/ ueber-uns/portfolio/segmente/.

Martin Bruckner. Modellierung der Fußgängerdynamik im universitären Betrieb mit Hilfe von Zellulären Automaten in der Programmiersprache JAVA. Master's thesis, Vienna University of Technology, Vienna, Austria, 2009.

Bundesministerium für Wissenschaft und Forschung. Datawarehouse Hochschulbereich, 2012. URL www.bmwf.gv.at/unidata.

Hans-Joachim Bungartz, Stefan Zimmer, Martin Buchholz, and Dirk Pflüger. Modellbildung und Simulatiuon - Eine anwendungsorientierte Einführung. eXamen.press. Springer, 2009.

Edmund Kieran Burke and Sanja Petrovic. Recent research directions in automated timetabling. European Journal of Operational Research, 140:266-280, 2002. 
Christian Burtscher, Pier-Paolo Pasqualoni, and Alan Scott. Das österreichische Universitätssystem im Schatten zweier Gesetze. In Heike Welte, Manfred Auer, and Claudia Meister-Scheytt, editors, Management von Universitäten - Zwischen Tradition und (Post-)Moderne, pages 137 - 164. Hamp, München, Mering, 2006.

Michael Carter. A comprehensive course timetabling and student scheduling system at the university of waterloo. In Edmund Kieran Burke and Wilhem Erben, editors, Practice and Theory of Automated Timetabling III, volume 2079 of Lecture Notes in Computer Science, pages 64-82. Springer Berlin, Heidelberg, 2001. ISBN 978-3-540-42421-5. URL http://dx.doi.org/10. 1007/3-540-44629-X_5.

Michael Carter and Gilbert Laporte. Recent developments in practical course timetabling. In Edmund Kieran Burke and Michael Carter, editors, Practice and Theory of Automated Timetabling II, volume 1408 of Lecture Notes in Computer Science, pages 3-19. Springer Berlin Heidelberg, 1998. ISBN 978-3-540-64979-3. URL http://dx.doi.org/10.1007/BFb0055878. $10.1007 / \mathrm{BFb} 0055878$.

Peter P. Chen. The entity-relationship model: Toward a unified view of data. ACM Transactions on Database Systems (TODS), 1(1):1-36, March 1976.

Peter P. Chen. Software Pioneers: Contributions to Software Engineering, chapter Entity-Relationship Modeling: Historical Events, Future Trends, and Lessons Learned, pages 296-310. Lecturing Notes in Computer Sciences. Springer-Verlag, Berlin, Germany, June 2002.

James P. Crutchfield, Melanie Mitchell, and Rajarshi Das. The evolutionary design of collective computation in cellular automata. Working Paper 98-09080, Santa Fe Institute, 1998.

Herbert Danner. Ökologische Wärmedämmstoffe im Vergleich 2.0. Landeshauptstadt München, Referat für Gesundheit und Umwelt, June 2010.

Indraneel Das and John Dennis. Normal-boundary intersection: An alternate method for generting pareto optimal points in multicriteria optimization problems. NASA Contractor Report 201616, ICASE, November 1996.

Martin Dehli. Wie energiesparend sind moderne Heizkessel? http://energiefakten.de, September 2010.

Jean-Luc Doumont. The cognitive style of PowerPoint: Slides are not all evil. Technical Communication, 52.1:64-70, February 2005.

Nancy Duarte. Slide:ology. O'Reilly Media, Sebastopol, CA, 2008.

Nancy Duarte. Resonate. John Wiley \& Sons, Hoboken, New Jersey, 2010.

Franziska Dzugan. TU Wien steht kurz vor der Pleite. Profil, April 2012.

Štefan Emrich. Comparison of mathematical models and development of a hybrid approach for the simulation and forecast of influenza epidemics within heterogeneous populations. Diploma Thesis. TU Vienna, 2007. 
Štefan Emrich, Felix Breitenecker, Günther Zauner, and Niki Popper. Simulation of influenza epidemics with a hybrid model - combining cellular automata and agent based features. In V. Luzar-Stiffler, V. Hljuz Dobric, and Z. Bekic, editors, Proceedings of the ITI 2008 30th Int. Conf. on Information Technology Interfaces, pages 709-714, Zagreb, Croatia, June 2008. SRCE University Computing Centre University of Zagreb.

Štefan Emrich, Sanja Zerlauth, Martin Bruckner, Niki Popper, Shabnam Tauböck, Dietmar Wiegand, and Felix Breitenecker. A simulation-based approach for control-optimization of lecture-room management. In M. Snorek, Z. Buk, M. Cepek, and J. Drchal, editors, Proceedings of the 7th Congress on Modelling and Simulation, volume 2, pages 1091-1097, 2010.

Štefan Emrich, Dietmar Wiegand, Felix Breitenecker, Marijana Srećković, Alexandra Kovacs, Benjamin Rozsenich, Salah Alkilani, and Niki Popper. Efficient use of space over time - deployment of the MoreSpace-tool. In Inge Troch and Felix Breitenecker, editors, 7th Vienna International Conference on Mathematical Modelling, volume 7 of Mathematical Modelling, pages 12071212. International Federation of Automatic Control, 2012.

Štefan Emrich, Dietmar Wiegand, and Alexandra Kovacs. A mathematical simulation tool for increased space utilization efficiency. To be published in Proceedings of EFMC 2013 (Prague, May 2013), May 2013.

European Commission. Towards a comprehensive climate change agreement in Copenhagen. Communication from the Commission to the European Parliament, the Council, the European Economic and Social Committee and the Committee of the Regions, $\operatorname{COM(2009)} 39$ final, 28 January 2009a.

European Commission. IP/09/1733 - Commissioner Piebalgs welcomes political agreement on energy performance of buildings. Press release, November 2009b.

Brad Ewing, David Moore, Steven Goldfinger, Anna Oursler, Anders Reed, and Mathis Wackernagel. Ecological Footprint Atlas 2010. Global Footprint Network, Oakland, CA, USA, October 2010a.

Brad Ewing, Anders Reed, Alessandro Galli, Justin Kitzes, and Mathis Wackernagel. Calculation methodology for the national footprint accounts, 2010 edition. Oakland, CA, USA, 2010b.

Stephen Few. Show me the Numbers - Designing Tables and Graphs to Enlighten. Analytic Press, Oakland, CA, USA, 2004.

Stephen Few. Information dashboard design. O'Reilly, Sebastopol, CA, 2006.

Paul A. Fishwick, editor. Handbook of dynamic system modeling. Computer and information science series. Chapman \& Hall/CRC, 2007a.

Paul A. Fishwick. Dynamic modeling - introduction. Youtube-video, 2007b. URL http://www . youtube. com/watch?v=k0dnPwXo2ZE.

Nigel Gilbert and Pietro Terna. How to build and use agent-based models in social science. Mind \& Society, 1(1):57-72, March 2000. 
HEFCE. Estates management statistics project. Technical Report 99/18, Higher Education Funding Council for England, March 1999.

Albert O. Hirschman. The Rhetoric of Reaction: Perversity, Futility, Jeopardy. The Belknap Press of Harvard University Press, 1991.

Darrell Huff. How to lie with statistics. Pelikan Books, 1977.

Laure Itard, Frits Meijer, Evert Vrins, and Harry Hoiting. Building renovation and modernisation in Europe: State of the art review. Delft, Netherlands, 2008.

Nicholas R. Jennings. On agent-based software engineering. Artificial Intelligence, 117:277-296, 2000.

Nicholas R. Jennings, Katia Sycara, and Michael Wooldridge. A roadmap of agent research and development. Journal Autonomous Agents and Multi-Agent Systems, 1(1):7-38, 1998.

Karin Joeckle, Monika Schneiders, Thomas Sieverts, Marianne Koch, and Hartmut Chodura. Organizing space in time - discovering existing resources. In Norbert A. Streitz, Shin'ichi Konomi, and Heinz-Jürgen Burkhardt, editors, Cooperative Buildings: Integrating Information, Organization, and Architecture, volume 1370 of Lecture Notes in Computer Science, pages 208218. Springer Berlin Heidelberg, 1998. ISBN 978-3-540-64237-4. doi: 10.1007/ 3-540-69706-3_21. URL http://dx.doi.org/10.1007/3-540-69706-3_21.

A. R. Kansal, S. Torquato, G. R. Harsh, E. A. Chiocca, and T. S. Deisboeck. Simulated brain tumor growth dynamics using a three-dimensional cellular automaton. Journal of Theoretical Biology, 203, Issue 4:367-382, April 2000.

Michihiko Kasugai. Zeit als Element der Stadtplanung: dargestellt am Beispiel Japan. PhD thesis, Technische Hochschule Darmstadt, 1985.

Daniel Keim, Jörn Kohlhammer, Geoffrey Ellis, and Florian Mansmann, editors. Mastering the Information Age - Solving Problems with Visual Analytics. Eurographics Association, Bad Langensalza, Germany, 2010.

Justin Kitzes, Alessandro Galli, Sarah Rizk, Anders Reed, and Mathis Wackernagel. Guidebook to the national footprint accounts: 2008 edition. Oakland, CA, USA, 2008.

Konferenz Kantonaler Energiedirektoren und Energiefachstellen. Energiepolitische Strategie der Kantone: Teilstrategie "Gebäude" für die zweite Hälfte von EnergieSchweiz (2006 - 2011). Bern, Switzerland, April 2005.

Alexandra Kovacs, Štefan Emrich, and Dietmar Wiegand. Strategies and methods to improve space-utilisation within companies. To be published in Proceedings of EFMC 2013 (Prague, May 2013), May 2013.

Hermann Krallmann, Helmut Frank, Norbert Gronau, H. Boekhoff, A. Ghanei, D. Kirsch, M. Schönherr, and B. Wyssusek. Systemanalyse im Unternehmen. Oldenbourg, 3 edition, 1999. 
Gerhard Krause. Wien will der TU goldene Brücken bauen. Kurier, (22-012005):10, January 2005.

C. M. Macal and M. J. North. Tutorial on agent-based modelling and simulation. Journal of Simulation, 4:151-162, 2010.

Riccardo Mazza. Introduction to Information Visualization. Springer-Verlag, London, 2009.

Barry McCollum. A perspective on bridging the gap between theory and practice in university timetabling. In Edmund Kieran Burke and Hana Rudová, editors, Practice and Theory of Automated Timetabling VI, volume 3867 of Lecture Notes in Computer Science, pages 3-23. Springer Berlin, Heidelberg, Berlin Heidelberg, 2007.

Roger McHaney. Computer simulation: a practical perspective. Elsevier Ltd., 1991. ISBN 9780124841406. URL http://books.google.at/books?id= tZ19CeMJXAkC.

McKinsey. Pathway to World-Class Energy Efficiency in Belgium, 2009.

Priska Mebes, Dietmar Wiegand, Veronika Pichler, and Karl Giger. Real Estate und Facility Management - neue Anwendungsbereiche für diskrete ereignisgesteuerte Simulationen. In Matthias Becker and Helena Szczerbicka, editors, ASIM 2006 - 19.Symposium Simulationstechnik. Universität Hannover, 2006.

J. Mendling, H. A. Reijers, and J. Cardoso. What makes process models understandable? In Gustavo Alonso and Peter Dadam und Michael Rosemann, editors, Business Process Management, volume 4714 of Lecture Notes in Computer Science, pages 48-63. Springer Berlin Heidelberg, 2007.

Achille Messac, Cyriaque Puemi-Sukam, and Emanuel Melachrinoudis. Aggregate objective functions and pareto frontiers: Required relationships and practical implications, 2000 .

George Miller. The magical number seven, plus or minus two: some limits on our capacity for processing information. Psychological Review, 63(2):81-97, 1956.

S. A. MirHassani and F. Habibi. Solution approaches to the course timetabling problem. Artificial Intelligence Review, pages 1-17, 2011.

Melanie Mitchell. Complexity - A Guided Tour. Oxford University Press, New York, 2009.

Melanie Mitchell, James P. Crutchfield, and Rajarshi Das. Evolving cellular automata with genetic algorithms: A review of recent work. In Proceedings of the First International Conference on Evolutionary Computation and Its Applications (EvCA'96), Moscow, Russia, 1996. Russian Academy of Sciences.

Object Management Group. Business process modeling notation, v1.1. Technical report, Object Management Group, 2008. 
H. Ottomann. Wussten Sie, dass Büroflächen nur zu fünf Prozent genutzt werden? Es ist Zeit! Mensch \& Büro, 2:28-30, 1994.

Sylvain Piechowiak, Jingxua Ma, and René Mandiau. An open interactive timetabling tool. In Edmund Kieran Burke and Michael Trick, editors, Practice and Theory of Automated Timetabling V, volume 3616 of Lecture Notes in Computer Science, pages 34-50. Springer Berlin, Heidelberg, Berlin Heidelberg, 2005.

Duncan Pollard, Rosamunde Almond, Emma Duncan, Monique Grooten, Lisa Hadeed, Barney Jeffries, and Richard McLellan, editors. Living Planet Report 2010: Biodiversity, biocapacity and development. WWF, Gland, Switzerland, 2010.

Selina Melanie Prirsch. MoreSpace - Potenziale zur Intensivierung der Raumnutzung über die Zeit bei Hochschulgebäuden in Österreich, Deutschland und der Schweiz. Master's thesis, Vienna University of Technology, March 2012.

Markus Rabe, Sven Spieckermann, and Sigrid Wenzel. Verifikation und Validierung für die Simulation in Produktion und Logistik - Vorgehensmodelle und Techniken. Springer, Berlin Heidelberg, Deutschland, 2008.

Hans-Gerd Ridder. Universitäten zwischen Eigensinn und strategischer Orientierung. In Heike Welte, Manfred Auer, and Claudia Meister-Scheytt, editors, Management von Universitäten - Zwischen Tradition und (Post-)Moderne, pages 101 - 113. Hamp, München, Mering, 2006.

Bertrand Russel. In Praise of Idleness. Routledge Classics, 2004.

Thomas Sauerbier. Theorie und Praxis von Simulationssystemen - Eine Einführung für Ingenieure und Informatiker. Vieweg, 1999.

H. R. Schalcher. Systems Engineering. ETH Zürich Departement Bau, Umwelt und Geomatik, 2008.

Thomas C. Schelling. Micromotives and Macrobehaviour. W. W. Norton \& Company; Revised edition (October 17, 2006), revised edition edition, October 2006.

Günter Schneckenreither, Patrick Einzinger, and Christoph Urach. Cellular automata - mathematical formalism. Workgroup-internal definition (Vienna University of Technology), 2013.

James C. Scott. Seeing Like a State: How Certain Schemes to Improve the Human Condition Have Failed. Yale Univ. Press, New Haven, 1998.

Ben Shneiderman. The eyes have it: A task by data type taxonomy for information visualizations. In P. van Zee, M. Burnett, and M. Chesire, editors, Proceedings of the 1996 IEEE Symposium on Visual Languages, IEEE Computer Society Press, pages $336-343$. IEEE, 1996.

Peter Skalicky. autonome Universität = autonome Entscheidung. TU—frei.haus, 3:3, June 2007. 
S. Solomon, D. Qin, M. Manning, Z. Chen, M. Marquis, K.B. Averyt, M. Tignor, and H.L. Miller, editors. IPCC, 2007: Summary for Policymakers. Climate Change 2007: The Physical Science Basis. Contribution of Working Group I to the Fourth Assessment Report of the Intergovernmental Panel on Climate Change. Cambridge University Press, Cambridge, UK and New York, USA, 2007.

Shabnam Michele Tauböck. Integration of Agent Based Modelling in DEVS for Utilisation Analysis: The MoreSpace Project at TU Vienna. PhD thesis, Vienna University of Technology, 2010.

TLB. The Living Bible. Tyndale House Bibles, 1971.

Edward R. Tufte. The Visual Display of Quantitative Information. Graphics Press, Cheshire, Connecticut, 6th printing edition, 1986.

Edward R. Tufte. The Cognitive Style of PowerPoint: Pitching Out Corrupts Within. Graphics Press LLC, Cheshire, Connecticut, 2nd edition, January 2006 .

H. Ulrich and G. Probst. Anleitung zum ganzheitlichen Denken: Ein Brevier für Führungskräfte. Verlag Paul Haupt, 1991.

Hans Ulrich. Systemorientiertes Management - Das Werk von Hans Ulrich. Stiftung zur Förderung systemorientierter Managementlehre, 2001.

Thomas Vahlenkamp. Kosten und Potentiale der Vermeidung von Treibhausgasemissionen in Deutschland: Sektorperspektive Gebäude. Technical report, McKinsey \& Company, Inc, 2007.

Verein Deutscher Ingenieure. Simulation von Logistik-, Materialfluß-, und Produktionssystemen - Begriffsdefinitionen. VDI 3633, 1996.

Vienna University of Technology. Rechnungsabschluss 2007. Technical report, Vienna University of Technology, 2007.

Vienna University of Technology. Rechnungsabschluss 2011. Technical report, Vienna University of Technology, 2011.

Colin Ware. Information Visualization: Perception for Design. Morgan Kaufmann, San Francisco, CA, 2004.

S. A. White. Introduction to BPMN, 2004. URL http://www.omg.org/bpmn/ Documents/Introduction_to_BPMN.pdf.

Thomas Wiedmann and Jan Minx. A definition of 'carbon footprint'. In C. C. Pertsova, editor, Ecological Economics Research Trends, pages 1-11. Nova Science Publishers, Hauppauge NY, USA, 2008.

D. Wiegand, P. Mebes, and V. Pichler. Event based simulations: Enabling improved development planning and partnership. In M. Schrenk, V. V. Popovich, and J. Benedikt, editors, REAL CORP 007: To Plan Is Not Enough. Strategies, Concepts, Plans, Projects and their Successful Implementation in Urban, Regional and Real Estate Development, pages 1723, Wien/Schwechat, Austria, May 2007a. CORP. 
Dietmar Wiegand. 5 Thesen zur nachhaltigen Entwicklung gebauter Umwelt / Facilities Development. In R. Köhler, editor, Schweizer Energiefachbuch 2008 - Nachhaltig Planen, Bauen und Betreiben., pages 122-125. Roland Köhler; KünzlerBachmann Medien AG, 2007.

Dietmar Wiegand. Ökologisch bauen und effizient nutzen - Ökologie ist auch die dringend notwendige Intensivierung der Raumnutzung über die Zeit. Green Life, Der Standard, 1:26-28, 2012.

Dietmar Wiegand, Priska Mebes, and Veronika Pichler. Discrete Event Simulations für die verbesserte strategische Planung und das Management von Facilities. In S. Wenzel, editor, Simulation in Produktion und Logistik 2006 - Tagungsband zur 12. ASIM Fachtagung in Kassel, 2006.

Dietmar Wiegand, Priska Mebes, and Veronika Pichler. Event based simulations: enabling improved lifecycle and risk management of facilities. In H. Schalcher and T. Wehrmüller, editors, 6th EuroFM Research Symposium - Conference Papers, pages 55-64. EuroFM, 2007b.

Dietmar Wiegand, Priska Mebes, and Veronika Pichler. Event-based simulations: Enabling improved lifecycle and risk management of facilities. SNE Simulation News Europe, 18(3-4):21-26, 2008.

Sam Williams. Unnatural selection. Technology Review, February 2005.

Dieter A. Wolf-Gladrow. Lattice-gas cellular automata and lattice Boltzmann models - an introduction. Springer Berlin, Heidelberg, 2000.

Stephen Wolfram. A New Kind of Science. Wolfram Media, 2002.

Evelyn Woplatek. Der muttersprachliche Unterricht in der Sekundarstufe: Eine Analyse mit Schwerpunkt auf Bosnisch/Kroatisch/Serbisch. Diploma Thesis. Universität Wien, 2010.

Ute Wotron. Pfuschen auf höchstem Niveau. Der Standard, 2009.

Bernard P. Zeigler. DEVS today: recent advances in discrete event-based information technology. In 11th IEEE/ACM International Symposium on Modeling, Analysis and Simulation of Computer Telecommunications Systems (MASCOTS'03), pages 148-161, 2003.

H. Zitter, K. Friedl, and M. Aschauer. Von 2 auf 20 Prozent - Optimierungspotentiale im Facility Management, September 2011. URL http://www. moo-con.com/Veranstaltungen/Rueckblick/Von-2-auf-20-Prozent:

-Die-Optimierungspotenziale-im-FM/103/51/de. 


\section{Curriculum VitaE}

Personal information

Name:

Date of birth:

Štefan Emrich

Place of birth:

30.06.1981

Nationality:

Vienna

Austrian

\section{Academic career}

Oct. 2008 - Aug. 2013

Oct. 2007 - Sept. 2008

since Oct. 2007

Feb. 2007 - Aug. 2007

Sept. 2004 - Jan. 2005

Oct. 2000 - June 2007

\section{Research projects}

since 2010

since 2008

$2007-2008$

$2006-2007$
Project assistant at the Chair of Real Estate Development and Management of Vienna University of Technology (TU Vienna)

Visiting scientist at the Institute of Construction and Infrastructure Management of the Swiss Federal Institute of Technology Zurich (ETH Zürich)

Doctoral program in technical sciences, TU Vienna Undergraduate research assistant at the Institute of Analysis and Scientific Computing, TU Vienna

Study abroad at the "Faculty for Preservation of the environment" at the Perm State Technical University (PSTU), Perm/Russia

Studies of technical mathematics, TU Vienna

INFO, Interdisziplinäre Forschung zur Energieoptimierung in Fertigungsbetrieben www.projekt-info.at (TU Vienna)

MoreSpace, dynamic simulation and statistical analysis for a increased efficiency in room utilization www.morespace.at (TU Vienna)

BUSSARD - Business and Services at Remote Destinations, Improvement of the workforce- and workflow-management of facility management providers (ETH Zürich)

Mathematical modeling of influenza epidemics within urban populations (TU Vienna) 


\section{Professional experience}

April 2004 - April 2006

August 2003

July, August 2002

Oct. 2000 - Sept 2008

Sept. 1999 - June 2000

Languages

\section{Computer skills}

\section{Other academic activities}

September $22-24,2010$

April 7 - 9, 2010

February $11-13,2009$

January $17-22,2008$
Secretary of the Youth of European Nationalities (voluntary)

Traineeship at "Datasystems Austria", Großwarasdorf, Austria

Traineeship at "Datasystems Austria", Großwarasdorf, Austria

Networkadministrator at "AHF GmbH", Großwarasdorf, Austria

Radio chat-show host and journalist at "Antenne 4" (freelance), Pinkafeld, Austria

$\begin{array}{ll}\text { German } & \text { C2 } \\ \text { English } & \text { C2 } \\ \text { Burgenland Croatian } & \text { C2 } \\ \text { Bosnian, Croatian, Serbian } & \text { C1 } \\ \text { Russian } & \text { B1 } \\ \text { Spanish } & \text { A1 }\end{array}$

MATLAB, LaTeX, R, AnyLogic, Enterprise Dynamics, MS Office, Open Office, iWork, Adobe Photoshop, Adobe InDesign, Widows, Ubuntu, MacOS

Session chair at BauSIM Conference, Vienna University of Technology

Invited presentation at Junior Scientist Conference, Vienna University of Technology

Organizing committee of MATHMOD Conference, Vienna University of Technology

Visiting lecturer at University of Business and

Technology in Pristina, Kosovo 